\title{
MÉTODOS DE MONTE CARLO EM ANÁLISE DE SOBREVIVÊNCIA
}

\author{
Vicente Garibay Cancho
}

TESE APRESENTADA

$\mathrm{AO}$

INSTITUTO DE MATEMÁTICA E ESTATÍSTICA

DA

UNIVERSIDADE DE SÃO PAULO

PARA

OBTENÇÃO DO GRAU

DE

DOUTOR EM ESTATÍSTICA

Área de Concentração: Estatística

Orientador: Prof. Dr. Heleno Bolfarine

Durante a elaboração deste trabalho, o autor recebeu apoio financeiro do $C N P q$

- São Paulo, setembro de 1999 - 


\section{MÉTODOS DE MONTE CARLO EM ANÁLISE DE SOBREVIVÊNCIA}

Este exemplar corresponde à redação final

da tese devidamente corrigida e

defendida por Vicente Garibay Cancho

e aprovada pela comissão julgadora.

São Paulo, 8 de setembro de 1999.

Banca examinadora:

- Prof. Dr. Heleno Bolfarine (Presidente) - IME - USP

- Prof. Dr. Francisco Louzada - Neto - UFSC.

- Prof. Dr. Helio Migon - UFRJ.

- Prof. Dr. Jorge Alberto Achcar - ICMC - USP.

- Prof. Dr. Reinaldo Boris Arellano Valle- PUCC-Chile. 
A mis padres Rosendo e Eulogia (em memoria) A mis hijos José e Daniel A mi amada esposa Gladys 


\section{AGRADECIMENTOS}

A Deus, por mais esta realização.

Ao meu orientador, Professor Heleno Bolfarine, pela oportunidade que tive de ter trabalhado mediante sua orientação segura e dedicada.

Aos Professores Jorge Alberto Achcar e Josemar Rodrigues pelo apoio que me brindaram. Aos membros da comissão julgadora, em especial ao Prof. Fransisco Louzada-Neto, pela observação de imprecisões e erros, na versão preliminar deste trabalho e por suas sugestões. Aos professores do Departamento de Estatística do IME-USP, por minha formação.

Aos meus familiares, que sempre me incentivaram a continuar, principalmente nos momentos difíceis e especialmente a minha cunhada Elena.

A todos meus colegas que em forma direta ou indireta contribuíram para a finalização deste trabalho.

Ao $\mathrm{CNPq}$, pelo apoio financeiro que permitiu realizar meus estudos.

Ao COSEAS, pela bolsa moradia.

E em especial, a minha esposa Gladys e meus filhos José Luis e Daniel Vincent.

Obrigados a todos. 


\section{RESUMO}

O principal objetivo deste trabalho é desenvolver técnicas Monte Carlo via Cadeias de Markov para alguns modelos especiais considerados na Teoria da Confiabilidade e na Análise de Sobrevivência. Uma metodologia Bayesiana é desenvolvida para o modelo Weibull-exponenciada proposto por Mudholkar et al. (1995). Uma extensão deste modelo é também considerada adicionando covariávéis ao modelo originalmente proposto por Mudholkar et al. (1995). Reportamos um estudo comparativo do modelo proposto por Mudholkar et al. (1995) com várias famílias de distribuições usadas no ajuste de dados de tempos de vida com função de taxas de falha do tipo "bathtub". Finalmente desenvolvemos um procedimento de análise Bayesiana para o modelo de mistura exponencial com ou sem covariáveis e, comparamos tal análise com os procedimentos clássicos de análise. Consideramos a distribuição Weibull-exponenciada para o ajuste de dados de tempo de vida na presença de indivíduos imunes e estudamos o uso deste modelo no teste de adequaçào do modelo de mistura Weibull e exponencial considerando as abordagens clássica e Bayesiana. 


\section{ABSTRACT}

The main object of this study is to develop Markov Chain Monte Carlo techniques in some special models typically considered in reliability theory and survival analysis. A Bayesian methodology is developed for the exponentiated-Weibull model proposed in Mudholkar et al. (1995). An extension of this model is also considered by adding covariates to the model originally proposed in Mudholkar et al. (1995). Are of the special features of the exponentiated-Weibull model is that it allows fitting bathtub failure rate models to data sets. Numerical studies are reported comparing several methods for fitting bathtub models to data. Next, we consider a Bayesian analysis for the exponential and Weibull mixture models for incorporating long-term survival data to the model. Comparisons with the classical maximum likelihood methodology are reported and extensions considered by using the exponentiated-Weibull model in this situation. 


\section{Conteúdo}

1 Introdução 1

1.1 Análise de Dados Tempo de Vida . . . . . . . . . . . . . . . . . 1

1.2 Análise Bayesiana para os modelos de tempo de vida . . . . . . . . . 3

1.2 .1 Teorema de Bayes . . . . . . . . . . . . . . . . . 3

1.2 .2 Algoritmos de simulação . . . . . . . . . . . . . . 5

1.2.3 Algumas considerações sobre seleção de modelos . . . . . . . . . . 8

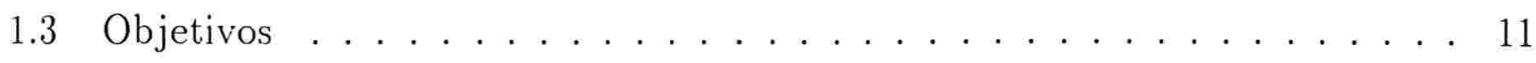

2 A familia de distribuições Weibull-exponenciada $\quad 12$

2.1 Introdução . . . . . . . . . . . . . . . . . . . . 12

2.2 A distribuição Weibull-exponenciada . . . . . . . . . . . . . . . . . 13

2.2 .1 Momentos .......................... 13

2.2.2 Função de sobrevivência e função risco . . . . . . . . . . . . . . 14

2.3 Análise clássica do modelo Weibull-exponenciada . . . . . . . . . . . 15

2.3.1 Estimação dos parâmetros por máxima verossimilhança . . . . . . . 15

2.3.2 Inferência sobre os parametros $\alpha, \theta$ e $\sigma \ldots \ldots \ldots \ldots$ 
2.3.3 Teste de bondade de ajuste . . . . . . . . . . . . 18

2.4 Análise Bayesiana do modelo Weibull-exponenciada . . . . . . . . . . . . . 19

2.4.1 Densidade conjunta a posteriori para os parâmetros do modelo Weibullexponenciada ........................... 19

2.4.2 Estimador de Bayes da função de sobrevivência e função risco . . . 21

2.4.3 Densidade conjunta a posteriori dos parâmetros do modelo Weibull 22

2.5 Exemplo de aplicação . . . . . . . . . . . . . . . . . . 23

3 Modelos de regressão Weibull-exponenciada 29

3.1 Introdução . . . . . . . . . . . . . . . . . . . . . 29

3.2 O Modelo log-Weibull-exponenciada . . . . . . . . . . . . . . . 30

3.3 O Modelo de regressão Weibul-exponenciada . . . . . . . . . . . . . . . 30

3.4 Análise clássica para os modelos de regressão Weibull-exponenciada . . . . 32

3.4.1 Estimação dos parâmetros por máxima verossimilhança . . . . . 32

3.4.2 Inferência sobre os parâmetros $\beta_{1}, \ldots, \beta_{p}, \delta$ e $\theta \ldots . \ldots 33$

3.4.3 Teste para modelo de regressão Weibull . . . . . . . . . . . . . 34

3.4.4 Teste para o modelo de regressão exponencial . . . . . . . . . . 35

3.5 Análise Bayesiana para o modelo de regressão Weibull-exponenciada . . . . 35

3.5.1 Densidade conjunta a posteriori dos parâmetros do modelo de regressão Weibull-exponenciada . . . . . . . . . . . . 36

3.5.2 Estimador de Bayes da função de sobrevivência no modelo de regressão Weibull-exponenciada . . . . . . . . . . . . 38 
3.5.3 Densidade conjunta a posteriori dos parâmetros do modelo de regressão Weibull . . . . . . . . . . . . . . . . . . . . 39

3.6 Exemplos de aplicação . . . . . . . . . . . . . . . . . . . . . . 40

3.6.1 Exemplo 1-Dados simulados . . . . . . . . . . . . . 40

3.6.2 Exemplo 2-Teste de vida de Isolantes . . . . . . . . . . . . . 44

4 Uso da distribuição Weibull-exponencida para dados com taxas de falha do tipo "bathtub"

4.1 Introdução . . . . . . . . . . . . . . . . . 53

4.2 Modelos . . . . . . . . . . . . . . . . . . . 54

4.2.1 A família de distribuições Weibull-exponenciada . . . . . . . 55

4.2 .2 A família de distribuições IDB $\ldots \ldots \ldots \ldots \ldots \ldots \ldots$

4.2 .3 A família de distribuições exponecial-potência . . . . . . . . . 5 56

4.2 .4 Misturas de distribuições Gama . . . . . . . . . . . . 57

4.3 Inferência Bayesiana $\ldots \ldots \ldots \ldots \ldots \ldots \ldots \ldots$

4.3.1 O Modelo Weibull-exponenciada . . . . . . . . . . . . 58

$4.3 .2 \quad$ O modelo IDB $\ldots \ldots \ldots \ldots \ldots \ldots \ldots$

$4.3 .3 \quad \mathrm{O}$ modelo exponencial-potência $\ldots \ldots \ldots \ldots$

4.3.4 Modelo de mistura distribuições de Gamas . . . . . . . . . . . . 61

4.4 Discriminação de modelos $\ldots \ldots$. . . . . . . . . . . . . 62

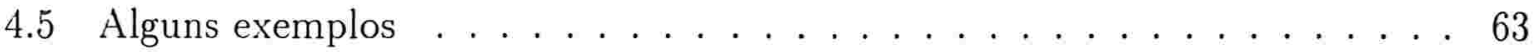

4.5 .1 Exemplo $1 \ldots \ldots \ldots \ldots \ldots \ldots \ldots \ldots \ldots \ldots \ldots$ 


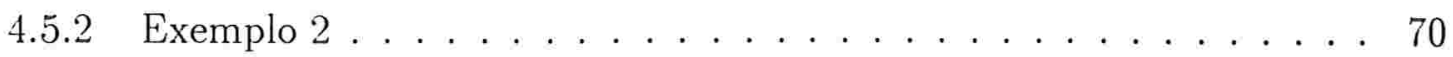

5 Comparação de dois modelos exponenciais com dados acelerados $\quad 78$

5.1 Introdução . . . . . . . . . . . . . . . . . . . . . . 78

5.2 Formulação do modelo . . . . . . . . . . . . . . . . . . . . . 79

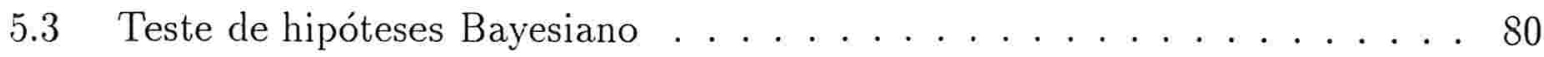

5.4 A função de verossimilhança $\ldots \ldots \ldots \ldots \ldots$. . . . . . . . . . 82

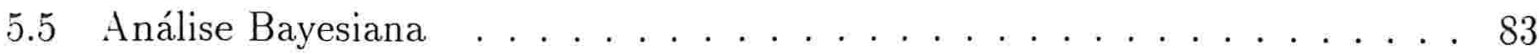

5.5 .1 DAP para os modelos $M_{1}$ e $M_{2} \ldots \ldots \ldots \ldots \ldots$

5.5 .2 Priori de Jeffreys $\ldots \ldots \ldots \ldots \ldots \ldots$

$5.5 .3 \quad$ Prioris Próprias . . . . . . . . . . . . . . . . . . . 91

$5.6 \quad$ Aplicação . . . . . . . . . . . . . . . . . . . . . . . . 93

6 Modelo de mistura exponencial 103

6.1 Introdução . . . . . . . . . . . . . . . . . . . . . . 103

6.2 Análise clássica do modelo de mistura exponencial . . . . . . . . . . . . . . 104

6.2.1 Estimadores de máxima verossimilhança . . . . . . . . . . 105

6.2.2 Inferência sobre os parâmetros $\lambda$ e $p \ldots \ldots \ldots \ldots$

6.2 .3 Um estudo de simulação . . . . . . . . . . . . . . . . . . 108

6.2.4 Comparação de proporções de indivíduos imunes entre grupos $\ldots 109$

6.2 .5 Estudo de simulação . . . . . . . . . . . . . . . . 110

6.3 Análise Bayesiana para modelos de mistura exponencial . . . . . . . . . 111 
6.3.1 Análise Bayesiana do modelo de mistura considerando a densidade a priori não informativa de Jeffreys . . . . . . . . . . . . . . 111

6.3 .2 Estudo de simulação . . . . . . . . . . . . . . . . . 115

6.4 Teste de hipóteses Bayesiano . . . . . . . . . . . . . . . . 117

6.4 Teste para $H_{0}: p=1 \ldots \ldots \ldots \ldots \ldots \ldots$

6.4 .2 Estudo de simulação . . . . . . . . . . . . . . . . . . 120

6.4.3 Comparação de proporções de sobreviventes . . . . . . . . . . . . 120

6.4.4 Teste para $H_{0}: p_{1}=\ldots=p_{G}=1 \ldots \ldots \ldots \ldots$

6.5 Exemplo ilustrativo . . . . . . . . . . . . . . . . 127

6.5.1 Análise clássica . . . . . . . . . . . . . . . 127

6.5 .2 Análise Bayesiana . . . . . . . . . . . . . . . . 130

7 Modelos de mistura exponencial com covariáveis $\quad 137$

7.1 Introdução . . . . . . . . . . . . . . . . . . 137

7.2 Análise clássica . . . . . . . . . . . . . . . . . 138

7.2.1 Estimadores de máxima verossimilhança . . . . . . . . . . . 140

7.2 .2 Inferência sobre os parâmetros $\alpha$ e $\beta \ldots . \ldots . \ldots . . \ldots 141$

7.3 Análise Bayesiana . . . . . . . . . . . . . . . . . . . . 142

7.3.1 Análise Bayesiana do modelo de mistura exponencial com covariáveis usando o método MCMC . . . . . . . . . . . . . . . . . 142

7.3.2 Estimador de Bayes do tempo médio de vida e para a proporção de sobreviventes dado um valor da covariável . . . . . . . . . . . . . 144

7.4 Aplicação . . . . . . . . . . . . . . . . . . . . . 144 
7.4.1 Exemplo 1-Dados simulados . . . . . . . . . . . . . . 145

7.4 .2 Exemplo 2-Dados de Kersey et al. 1987 . . . . . . . . . . . . 149

8 Modelo de mistura Weibull-exponenciada 153

8.1 Introdução . . . . . . . . . . . . . . . . . . . . . . 153

$8.2 \quad$ Modelo. . . . . . . . . . . . . . . . . . . 153

8.3 Uma análise clássica do modelo de mistura Weibull-exponenciada . . . . 154

8.3.1 Estimação dos parâmetros por máxima verossimilhança . . . . . . . 155

8.3.2 Inferências sobre os parâmetros $\alpha, \theta, \lambda$ e $p \ldots \ldots \ldots \ldots$

8.3.3 Teste do modelo de mistura Weibull . . . . . . . . . . 157

8.3.4 Teste para o modelo de mistura exponencial . . . . . . . . 158

8.4 L'ma análise Bayesiana para o modelo de mistura Weibull-exponenciada . . 158

8.4.1 Densidade a priori independentes . . . . . . . . . . . . . 159

8.4.2 Densidade conjunta a posteriori pâra os parâmetros do modelo de mistura Weibull-exponenciada . . . . . . . . . . . . . . 160

8.4.3 Densidade conjunta a posteriori para os parâmetros do modelo de mistura Weibull . . . . . . . . . . . . . . . . . 162

8.4.4 Teste de hipóteses Bayesiano . . . . . . . . . . . . . . 163

8.5 Exemplos de aplicação . . . . . . . . . . . . . . . . . . . . . . . . 164

8.5.1 Exemplo 1-Dados Simulados . . . . . . . . . . . . . . . 164

8.5.2 Exemplo 2-Dados de Kersey et al. $1987 \ldots \ldots$. . . . . . . . . 171 
Apêndices

A Critério de convergência

187

B Alguns programas

189 


\section{Capítulo 1}

\section{Introdução}

\subsection{Análise de Dados Tempo de Vida}

A análise estatística de dados de tempos de vida representa o principal interesse de duas áreas de estudo, a teoria de confiabilidade e, a análise de sobrevivencia. Na confiabilidade, temos como exemplos os teste de vida onde os dados utilizados referem-se ao tempo de falha de um ítem, enquanto que, na análise de sobrevivência os dados representam em geral o tempo de vida ou tempo de remissão de uma doença, de pacientes ou animais sob experimentação. Esses dados de tempo de vida são frequentemente dados censuradas, isto é, para alguns elementos em estudo não sabemos seu tempo exato de vida, mas apenas que excede um certo valor, já que não é possível esperar que todas unidades experimentais em teste falhem e é necessário que o experimento se encerre num determinado tempo fixo, ou ainda, que ele se encerre depois de um certo número de falhas tenha ocorrido.

$\mathrm{Na}$ análise de sobrevivência e confiabilidade alguns conceitos são de suma importância, para isto considere uma variável aleatória contínua não negativa $T$, representado o tempo de vida de um indivíduo ou dispositivo de uma população homogênea. A distribuição de probabilidade de $T$ pode ser representada por três formas equivalentes. 
A função de densidade que é definida por,

$$
f(t)=\lim _{\Delta t \rightarrow 0} p(t \leq T \leq t+\Delta t)=\frac{\partial F(t)}{\partial t}, t>0
$$

onde $F(t)=P(T \leq t)$ é a função de distribuição de $T$, a função de sobrevivência de $T$ definida por,

$$
S(t)=P(T \geq t)=\int_{t}^{\infty} f(x) d x
$$

onde esta função representa a probabilidade de um indivíduo (ou ítem) sobreviver pelo menos até o tempo $t$. Em alguns contextos, epecialmente aqueles envolvendo tempos de vida de ítens essa função é chamada de função de confiabilidade. Pode-se mostrar que $S(t)$ é uma função monótona decrescente, contínua, com $S(0)=1$ e $\lim _{t \rightarrow \infty} S(t)=0$.

Finalmente a função risco que especifica a taxa instantânea de falha ou morte no tempo $t$ de um indivíduo (ou ítem), dado que ele sobreviveu até o tempo $t$, e que é definida por

$$
h(t)=\lim _{\Delta t \rightarrow 0}=\frac{p(t \leq T \leq t+\Delta t \mid T \geq t)}{\Delta t}=\frac{f(t)}{S^{\prime}(t)} .
$$

A densidade $f(t)$ e a função de sobrevivência $S(t)$ são representações comuns da distribuição de uma variável aleatória. Enquanto, a função risco $h(t)$ é uma caracterização mais especializada, mas é particularmente útil na modelagem de dados de sobrevivência. Frequentemente em aplicações, tem-se disponível a informação de como a taxa de falha muda com o tempo, e estas informações podem ser usadas na modelagem de $h(t)$. Na literatura existem alguns modelos que são usados com bastante frequência (veja por exemplo Lawles, 1982; Collet, 1994). Alguns destes modelos são os modelos exponencial e Weibull. Contudo, esses modelos não incluem o caso de funções de taxa de falha em forma de "bathtub" ou unimodal. Para tais situações foram propostos muitos modelos paramétricos entre eles estão as distribuições $F$ geralizada de Printice (1975), gama generalizada de Stacy (1962) entre outros; não obstante a modelagem desses modelos é computacionalmente muito complicada na precença de dados censurados. Mudholkar et al. (1995) apresentam uma extenção da distribuiçào Weibull denominada Weibull-exponenciada que além de ter a caracteristica de ter funções de risco unimodal e em forma de "bathtub", inclue 
os modelos com funções risco monótonos. No Capítulo 2, apresentamos a distribuição Weibull-exponenciada e algumas de suas propriedades

\subsection{Análise Bayesiana para os modelos de tempo de vida}

$\mathrm{Na}$ análise de confiabilidade ou de sobrevivência, quando assumimos um modelo para os dados de tempos de vida, temos interesse em determinar o valor do parâmetro que caracteriza o modelo. Embora este parâmetro seja considerado fixo, não temos certeza do seu verdadeiro valor e assim procuramos uma forma de, através dos dados, expressar essa incerteza.

Uma alternativa é considerar uma distribuição para o parâmetro de interesse, que sob este ponto de vista é considerado uma variável aleatória (ver por exemplo Box e Tiao, 1973). Com esta formulação usamos o teorema de Bayes, que fornece um método para computar a distribuição do parâmetro, expressando nossa incerteza condicionada aos dado. Para análise de sobrevivência ou confiabilidade vários trabalhos utilizando abordagem Bayesiana já foram realizados (ver por exemplo, Achcar e Louzada-Neto, 1992; Rodrigues, Bolfarine e Louzada-Neto, 1993, entre outros).

\subsubsection{Teorema de Bayes}

Suponha que $\mathrm{t}=\left(t_{1}, \ldots, t_{n}\right)$ é um vetor de $n$ observações onde a distribuição de probabilidade $\pi(\mathbf{t} \mid \theta)$ depende de $p$ parâmetros. A quantidade $\theta=\left(\theta_{1}, \ldots, \theta_{p}\right)$ sob o ponto de vista Bayesiano, é considerado uma variável aleatória, representado caracteristicas que se deseja conhecer. Se $\theta$ tem uma distribuição de probabilidade (denominda distribuição a priori) $\pi(\theta)$, então,

$$
\pi(\mathrm{t} \mid \theta) \pi(\theta)=\pi(\mathrm{t}, \theta)=\pi(\theta \mid \mathrm{t}) \pi(\mathrm{t})
$$


A distribuição de $\theta$ dado as observações é dada por

$$
\pi(\theta \mid \mathrm{t})=\frac{\pi(\mathrm{t} \mid \boldsymbol{\theta}) \pi(\theta)}{\pi(\mathrm{t})},
$$

onde

$$
\pi(\mathrm{t})=c^{-1}=\int_{\Theta} \pi(\mathbf{t} \mid \theta) \pi(\theta) d \theta .
$$

Observe que $\pi(\mathrm{t})$ é a esperança matemática de $\pi(\mathrm{t} \mid \theta)$ em relação a densidade a priori $\pi(\theta)$.

Se denotamos a função de verossimilhança $\pi(\mathbf{t} \mid \theta)$ por $L(\theta)$, pelo teorema de Bayes podemos escrever (1.1) como

$$
\pi(\theta \mid \mathrm{t})=c L(\theta) \pi(\theta)
$$

onde $c$ é a constante normalizadora de $\pi(\theta \mid \mathbf{t})$.

Portanto a densidade a posteriori (1.1) pode ser escrita como

$$
\pi(\theta \mid \mathrm{t}) \propto L(\theta) \pi(\theta) .
$$

O procedimento de inferência Bayesiana baseia-se na distribuição a posteriori das componentes de $\theta$. Neste ponto surgem duas dificuldades, a primeira na obtenção da distribuição a posteriori marginal e a segunda no cálculo de momentos a posteriori de interesse. Em ambos casos é necessário a resolução de integrais que muitas vezes não apresentam uma solução análitica. Nesse caso, métodos numéricos (ver por exemplo, Naylor e Smith, 1982) ou métodos de aproximação de integrais, tal como o método de Laplace (ver por exemplo Tierney e Kadane, 1986 ) são necessários. Ocorre que para a aplicação deste último método é necessário determinar o valor que maximiza a função de densidade a posteriori e assim a medida que a dimensão do espaço paramétrico aumenta, aumentam as dificuldades para sua obtenção e a aplicação do método vai se tornando mais trabalhosa de ser implementada. Neste sentido, uma alternativa, é utilizar os algoritmos de simulação de Monte Carlo via Cadeias de Markov (MCMC) tais como os algoritmos de Gibbs (ver por exemplo, Gelfand e Smith,1990) e Metropolis-Hasting (ver por exemplo, 
Chib e Grenberg, 1995). Esse métodos permitem inclusive escolher densidades a priori sem prender a propriedade de conjugação.

Um outro elemento importante na inferência Bayesiana é a distribuição preditiva. Usualmente estamos interessados em fazer inferência sobre alguma observação futura, $s$, utilizando as observações passadas t. Tal inferência deve ser baseada na distribuição a posteriori de $s, \pi(s \mid \mathbf{t})$. Observamos que $\pi(s \mid \mathbf{t})$ pode ser obtida a partir da distribuição a posteriori conjunta de $s$ e $\theta$ por integração, ou seja,

$$
\pi(s \mid \mathbf{t})=\int \pi(s, \theta \mid \mathbf{t}) d \theta=\int \pi(s \mid \theta, \mathbf{t}) \pi(\theta \mid \mathbf{t}) d \theta,
$$

onde $\pi(s \mid \theta, \mathrm{t})$ em muitos modelos se reduz a $\pi(s \mid \theta)$.

\subsubsection{Algoritmos de simulação}

O algoritmo do amostrador de Gibbs (A.G.) e algoritmo de Metropolis-Hasting são um dos métodos de simulação de Monte Carlo via Cadeias de Markov (MCMC). O A.G. é uma técnica de MCMC para gerar amostras de uma densidade multivariada não normalizada. Os métodos de simulação MCMC atualmente têm sido utilizados na inferência Bayesiana (ver por exemplo, Gelfand e Smith, 1990 e Chib e Greenberg, 1995). Na inferência Bayesiana a distribuição estacionária desta Cadeia de Markov é a distribuição a posteriori. Geman e Geman (1984) apresentaram o algoritmo do A.G. em problemas de procesamento de imagens. Entretanto o método já havia sido descrito por Metropolis (1953) e posteriormente por Hasting (1970). Gelfand e Smith (1990) popularizaram o A.G. na aplicação do método na área de inferência Bayesiana.

Descrevemos brevemente o procedimento do amostrador de Gibbs e em geral o algoritmo de Metropolis-Hasting. Suponha que desejamos estimar $\pi\left(\theta_{1}, \ldots, \theta_{p} \mid D\right)$, densidade conjunta a posteriori do $\left(\theta_{1}, \ldots, \theta_{p}\right)$ dado os dados. $\mathrm{O}$ algoritmo considera que as densidades condicionais a posteriori de cada parâmetro $\theta_{i}$, dado todos os outros sejam conhecidas. Essas distribuições são denotados por $\pi\left(\theta_{1} \mid \theta_{2}, \ldots, \theta_{p}, D\right), \pi\left(\theta_{2} \mid \theta_{1}, \theta_{3}, \ldots, \theta_{p}, D\right), \ldots$, $\pi\left(\theta_{p} \mid \theta_{1}, \theta_{2}, \ldots, \theta_{p-1}, D\right)$. 
Com os valores iniciais $\theta_{1}^{(0)}, \ldots, \theta_{p}^{(0)}$ geramos

$$
\begin{array}{ccc}
\theta_{1}^{(1)} & \text { de } & \pi\left(\theta_{1} \mid \theta_{2}^{(0)}, \ldots, \theta_{p}^{(0)}, D\right), \\
\theta_{2}^{(1)} & \text { de } & \pi\left(\theta_{2} \mid \theta_{1}^{(1)}, \theta_{3}^{(0)}, \ldots, \theta_{p}^{(0)}, D\right), \\
\vdots & \vdots & \vdots \\
\theta_{p}^{(1)} & \text { de } & \pi\left(\theta_{p} \mid \theta_{1}^{(1)}, \theta_{2}^{(1)}, \ldots, \theta_{p-1}^{(1)}, D\right),
\end{array}
$$

com essa geração $\theta^{(1)}=\left(\theta_{1}^{(1)}, \ldots, \theta_{p}^{(1)}\right)^{\prime}$ 'substitui o valor inicial e continuamos iterando até a iteração $k$, onde se espera que a convergência da cadeia é alcançada. Sob certas condições de regularidade (veja, por exemplo Tierney,1994), essa cadeia de Markov converge para distribuição estacionária para $k$ suficientemente grande, isto é, $\left(\theta_{1}^{(k)}, \ldots, \theta_{p}^{(k)}\right)^{\prime}$ tem uma distribuição aproximadamente igual a $\pi\left(\theta_{1}, \ldots, \theta_{p} \mid D\right)$. Além disso, $\theta_{i}$ pode ser considerada como uma observação simulada de $\pi\left(\theta_{i} \mid D\right)$, a distribuição marginal de $\theta_{i}$ (veja, Geman e Geman, 1984). Se consideramos $s$ pontos iniciais independentes, podemos também replicar a iteração anterior $r$ vezes. Seja $\theta^{(i, s)}=\left(\theta_{1}^{(i, s)}, \ldots, \theta_{p}^{(i, s)}\right)^{\prime}$ denota a realização de $\theta$ obtida a partir da $i$-ésima iteração e da s-ésima réplica. Para estimar $\pi\left(\theta_{1}, \ldots, \theta_{p} \mid D\right)$, seus momentos de interesse e intervalos de credibilidade, pode-se usar uma medida empírica atribuindo $1 / r$ de peso a cada $\left(\theta_{1}^{(k, s)}, \ldots, \theta_{p}^{(k, s)}\right), s=1, \ldots r$. Alternativamente, Gelman e Rubin (1992) sugerem usar essa medida empírica para todas as variáveis considerando a segunda metade de todas as iterações e réplicas.

Para o uso da convergência e escolha prática de $k$ e $r$, pode-se consultar Tanner e Wong(1987), Gelfand e Smith (1990), Casella e George (1991), e Gelman e Rubin (1992) e Cowles e Carlin (1996). Na prática, entretanto, ainda existe muita discussão sobre o minitoramento da convergência. Contudo, o método proposto por Gelman e Rubin (1992) o qual se baseia na técnica de análise de variância é a mais utilizada na inferência Bayesiana (veja por exemplo, Achcar e Períra., 1998).

Quando as densidades condicionais não são facilmente identificáveis, ou se a geração não-iterativa for muito complicada o algoritmo de Metropolis-Hasting (M.H.) pode ser usado. Descrevemos a seguir o algoritmo de M.H. Suponha que desejamos uma amostra 
da variável $\theta_{1}$ em (1.3) onde sua densidade condicional a posteriori não é conhecida. Assim, consideramos a densidade genérica

$$
\pi\left(\theta_{1} \mid \theta_{2}, \ldots, \theta_{p}, D\right)=\frac{L\left(\theta_{1}, \ldots, \theta_{p}\right) \pi\left(\theta_{1}, \ldots, \theta_{p}\right)}{\int L\left(\theta_{1}, \ldots, \theta_{p}\right) \pi\left(\theta_{1}, \ldots, \theta_{p}\right) d \theta_{1}}
$$

Vamos denotar por $f\left(\theta_{1}\right)$ a densidade condicional em (1.4) suprimindo as variáveis condicionantes por brevidade. Assim define-se o núcleo de transição $q\left(\theta_{1}, X\right)$ que leva $\theta_{1}$ a $X$. Se $\theta_{1}$ é um valor real com suporte $(-\infty, \infty)$, construiremos $q($.$) de tal forma que$ $X=\theta_{1}+\sigma Z$, sendo $Z$ uma variável aleatória com distribuição normal padrão (ou $t$ student) e $\sigma^{2}$ tem que refletir a variância condicional de $\theta_{1}$ em (1.4). Se $\theta_{1}$ é uma variável limitada com suporte no intervalo $(a, b)$, podemos usar uma transformação, tal como $\theta^{\prime}=\log \left(\frac{\theta_{1}-a}{b-\theta_{1}}\right)$, para levar $(a, b)$ em $(-\infty, \infty)$ e então usamos o núcleo de transição anterior na densidade de $\theta^{\prime}$. Alternativamente, podemos aplicar um método simples sem a necessidade de considerar uma transformação. Construimos o núcleo de transição $q($.) de modo que $X$ seja distribuída uniformemente em $(a, b)$ (veja, por exemplo Kuo et al. 1995). Outra forma de escolha, quando possivel, é explorar a forma de $f\left(\theta_{1}\right)$ para obter o núcleo de transição $q($.$) (veja Chib e Greenberg, 1995). Por exemplo, se f\left(\theta_{1}\right)$ pode ser dada por $f\left(\theta_{1}\right) \propto \Psi\left(\theta_{1}\right) h\left(\theta_{1}\right)$, onde $h\left(\theta_{1}\right)$ é uma densidade que pode ser amostrada e $\Psi\left(\theta_{1}\right)$ é uniformemente limitada, fazemos $q\left(\theta_{1}, X\right)=h\left(\theta_{1}\right)$. Outras possíveis escolhas podem ser encontradas em Chib e Greenberg (1995).

O algoritmo de M.H. pode ser descrito como segue:

(i) inicialize $\theta_{1}$ com um valor $\theta_{1}^{(0)}, j=0$;

(ii) gerar $X$ de $q\left(\theta_{1}^{(j)},.\right)$ e $u$ da distribuição uniforme $(0,1)$;

(iii) calcular $p=\min \left\{1, \frac{f(X) q\left(\theta_{1}^{(\jmath)}, X\right)}{f\left(\theta_{1}^{(J)}\right) q\left(X, \theta_{1}^{(J)}\right)}\right\}$;

(iv) se $u \leq p$; faça $\theta_{1}^{(j+1)}=X$, senão faça $\theta_{1}^{(j+1)}=\theta_{1}^{(j)}$;

(v) repita (ii)- (iv) até que a distribuição estacionária seja atingida. 
Observe que este algoritmo é definido usando a razão de dois valores obtidos de (1.4). Portanto, tem-se que conhecer a forma funcional da função de verossimilhança e a densidade a priori, mas, não a constante normalizador $A$.

\subsubsection{Algumas considerações sobre seleção de modelos}

A determinação do modelo se torna um problema fundamental da estatística. A literatura sobre a adequação ou checagem e seleção de modelos apresenta muitas abordagens, començando com a abordagem do fator Bayes. Várias modificações do fator de Bayes são apresentadas na literatura (veja por exemplo, Atikin, 1981; Berger e Perichi, 1992 ou Spiegelhater e Smith, 1982). Geisser e Eddy (1979) sugerem uma abordagem preditiva baseada em métodos de validação cruzada para obter o pseudo-fator de Bayes.

Considere uma escolha entre dois modelos paramétricos cada um denotado por sua densidade conjunta $f\left(\underline{t} \mid \boldsymbol{\theta}_{i}, M_{i}\right)$ ou por sua função de verossimilhança $L\left(\boldsymbol{\theta}_{i} \mid \underline{t}, M_{i}\right), i=1,2$. Suponha que $w_{i}$ é a probabilidade de selecionar o modelo $M_{i}, i=1,2$ e $f\left(\underline{t} \mid M_{i}\right)$ é a distribuição preditiva (verossimilhança preditiva) do modelo $M_{i}$, que é definida por,

$$
f\left(\underline{t} \mid . M_{i}\right)=\int f\left(\underline{t} \mid \theta_{i}, M_{i}\right) \pi\left(\theta_{i} \mid M_{i}\right) d \theta_{i},
$$

onde $\pi\left(\theta_{i} \mid M_{i}\right)$ é a densidade a priori sobre o modelo $M_{i}$. Se $\underline{t}_{0}$ denota os dados observados, então escolhemos o modelo que tem maior valor $w_{i} f\left(\underline{t}_{0} \mid M_{i}\right)$.

Frequentemente considera-se $w_{i}=0.5, i=1,2$ e o fator de Bayes de $M_{1}$ com respeito a $M_{2}$ é definido como

$$
B_{12}=\frac{f\left(\underline{t}_{0} \mid M_{1}\right)}{f\left(\underline{t}_{0} \mid M_{2}\right)}
$$

A veressimilhança preditiva (1.5) pode ser aproximada por Monte Carlo usando $S$ amostras geradas a partir da densidade a priori $\pi\left(\theta_{i} \mid M_{i}\right)$, isto é,

$$
\hat{f}\left(\underline{t} \mid M_{i}\right)=\frac{1}{S} \sum_{s=1}^{S} f\left(\underline{t} \mid \theta_{i}^{(s)}, M_{i}\right),
$$

onde $\theta_{i}^{(s)}$ é a $s$-ésima amostra do vetor $\theta_{i}$. 
Podemos notar que, caso a distribuição a priori $\pi\left(\theta_{i} \mid M_{i}\right)$ seja imprópria, o que pode acontecer quando consideramos densidades a priori não informativas, $f\left(D \mid M_{i}\right)$ também será imprópria e consequentemente (1.6) não pode ser usada como critério de comparação, uma vez que não se tem uma razão de probabilidades. Esse fato pode restringir o uso do fator de Bayes à classe das prioris próprias, ou ainda a modelos razoavelmente simples, o que em geral não ocorre na prática, principalmente com relação a modelos de sobrevivência ou confiabilidade.

No sentido de superar restrições associad $\phi \phi_{s}$ ao fator de Bayes, muitos trabalhos recentemente publicados sugerem mudanças em (1.6) (veja por exemplo, Gelfand e Dey, 1994). O uso da distribuições preditivas em alguma forma tem sido reconhecida como a correta abordagem Bayesiana para a seleção de modelos. Particularmente, Box (1980) argumenta que densidades a posteriori devem ser usadas para a estimação dos parâmetros condicional a adequabilidade do modelo, enquanto que densidades preditivas devem ser usadas para a análise da adequabilidade dos modelos na presença dos dados. Na avaliação de dois modelos quaisquer as densidades preditivas podem ser comparadas enquanto que as densidades a posteriori nem sempre, conforme visto acima.

A densidade preditiva que foi utilizada extensivamente em trabalhos recentes de Gelfand e Dey (1994) e Gelfand, Dey e Chang (1992) é a densidade de validação cruzada dada por,

$$
f\left(t_{r} \mid D_{(r)}\right)=\frac{f(D)}{f\left(D_{(r)}\right)}=\int f\left(t_{r} \mid \theta, D_{(r)}\right) \pi\left(\theta \mid D_{(r)}\right) d \theta
$$

onde $D_{(r)}=\left(t_{1}, \ldots, t_{r-1}, t_{r+1}, \ldots, t_{n}\right)$ e $\pi\left(\theta \mid D_{(r)}\right)$ é a correspondente densidade a posteriori baseada em $D_{(r)}$. Essa densidade pode ser chamada de densidade preditiva condicional ordenada (CPO).

Na aproximação de validação cruzada, um único ponto é deletado, e este procedimento é uma rotina padrão em análise de regressão clássica e muitas técnicas de diagnósticos adotam esse ponto de vista. Isto também é estabelecido na literatura Bayesiana por Stone (1974). 
Para obter uma estimativa da densidade preditiva de validação cruzada observe que

$$
\begin{aligned}
f\left(t_{r} \mid D_{(r)}\right) & =\frac{f(D)}{f\left(D_{(r)}\right)}=\frac{\int \frac{f(D \mid \theta) \pi(\theta) 4}{\pi(\theta \mid D) f(D)} \cdot \pi(\theta \mid D) d \theta}{\int \frac{f\left(D_{(r)} \mid \theta\right) \pi(\theta)}{\pi(\theta \mid D) f(D)} \cdot \pi(\theta \mid D) d \theta} \\
& =\frac{1}{\int \frac{1}{f\left(t_{r} \mid D_{(r)}, \theta\right)} \pi(\theta \mid D) d \theta} .
\end{aligned}
$$

Daí, por aproximação de Monte Carlo em (1.9) obtem-se a estimativa

$$
\hat{f}\left(t_{r} \mid D_{(r)}\right)=B\left(\sum_{i=1}^{B} \frac{1}{f\left(t_{r} \mid D_{(r)}, \theta_{i}\right)}\right)^{-1} .
$$

onde $B$ é o tamanho da amostra a posteriori de $\theta$.

Se $t_{r}, r=1, \ldots, n$, são condicionalmente independentes dado $\theta, f\left(t_{r} \mid D_{(r)}, \theta_{i}\right)$ simplificase para $f\left(t_{r} \mid \theta_{i}\right)$. Observe que esta quantidade pode ser considerada como a $r$-ésima componente da função de verossimilhança ou como a densidade condicional de $t_{r}$ dado $\theta_{i}$. Logo, (1.10) é a média harmônica das componentes da função verossimilhança ou média harmônica da densidade condicional avaliada na amostra a posteriori.

Podemos utilizar as estimativas $c_{r}(l)=\hat{f}\left(t_{r} \mid D_{(r)}\right)$ na seleção dos modelos, considerando o gráfico dos valores $c_{r}(l)$ contra o número de observações $r(r=1, \ldots, n)$ para os diferentes modelos; valores maiores (em média) indica o melhor modelo. Também pode-se considerar para escolher o modelo que maximiza $c(l)=\prod_{r=1}^{n} c_{r}(l)$, onde $l$ é o indexador do modelo.

Alternativamente, podemos considerar o gráfico da razão dos CPO's (ou log da razão dos CPO's) dos diferentes modelos contra o número de observações para selecionar o melhor modelo; valores da razão maiores que um (ou positivos) indicam a preferência do primeiro modelo.

Geisser e Eddy (1979) sugere que o produto das densidades preditivas $\prod_{r=1}^{n} f\left(t_{r} \mid D_{(r)}, M_{l}\right)$ pode ser usada como um indicador na seleção de modelos. Se temos dois modelos $M_{1}$ e 
$M_{2}$, temos que a razão,

$$
\frac{\prod_{r=1}^{n} f\left(t_{r} \mid D_{(r)}, M_{1}\right)}{\prod_{r=1}^{n} f\left(t_{r} \mid D_{(r)}, M_{2}\right)}
$$

pode ser usada como uma aproximação do fator de Bayes, a qual denota-se por PSF $B_{12}$ (pseudo-fator de Bayes,) escolhendo-se o modelo $M_{1}$ se $P S F B_{12}>1$.

\subsection{Objetivos}

O principal objetivo deste trabalho é fazer análise Bayesiana para modelos de sobrevivência ou confiabilidade na ausência ou presença de indivíduos imunes utilizando os métodos de simulação via Cadeias de Markov com Monte Carlo.

Este trabalho está organizado em 8 Capítulos. O Capítulo 2 é dedicado à inferência Bayesiana considerando a distribuição Weibull-exponenciada para os tempos de sobrevivência e ao estudo de algumas propriedades dessa distribuição. No Capítulo 3 estendemos a família de distribuição Weibull-exponenciada para caso do modelos de regressão e apresentamos o procedimento de inferência clássica e Bayesiana para o modelo. No Capítulo 4 apresentamos um estudo comparativo da distribuição Weibull-exponenciada com as famílias de distribuições mais comumente usadas para modelar tempos de vida que apresentam função de taxa de falha do tipo "bathtub" desde uma perspectiva Bayesiana. No Capítulo 5 desenvolvemos uma análise Bayesiana considerando várias densidades a priori, para compararmos 2 populações sob condições aceleradas considerando a distribuição exponencial para os tempos de vida. No Capítulo 6 desenvolvemos o procedimento de inferência Bayesiana para os modelos de sobrevivência na presença de indivíduos imunes (modelos de mistura) considerando a distribuição exponencial para os tempos de vida. No Capítulo 7, apresentamos o procedimento de inferência Bayesiana para modelos de mistura exponencial na preģença de covariáveis. Finalmente no Capítulo $\&$ fazemos inferência clássica e Bayesiana para os modelos de mistura Weibull-exponenciada. 


\section{Capítulo 2}

\section{A família de distribuições Weibull-exponenciada}

\subsection{Introdução}

A família de distribuição Weibull é muito utilizada como um modelo para a distribuição de tempos de vida, tanto na área médica como na engenharia. Essa família de distribuições é adequada para modelar dados de tempo de vida com função risco monótonas ou constantes. Contudo, não é adequada para modelar situações onde a função de risco tem forma de banheira ou unimodal. Muitas famílias de distribuições foram intruduzidas, para modelar as situações anteriores, como por exemplo, a familia de distribuição $F$ generalizada de Prentice (1975), a distribuição gama generalizada de Stacy (1962) ou duas famílias introduzidas por Slymen e Lachenbruch (1984). Porém, a modelagem de dados de tempo de vida com essas famílias de distribuições são computacionalmente muito complicadas na precença de censura.

Mudholkar et al.(1995) apresentam uma extensão da distribuição Weibull denominada família de distribuições Weibull-exponenciadas (WE) em aplicações de confiabilidade e sobrevivência. A nova família é obtida ao introduzir um segundo parâmetro de for- 
ma $\theta$ adicional na função quantil da distribuição Weibull. Essa extensão, que contém distribuições com função de risco unimodal e em forma de "bathtub", também permite considerar uma ampla classe de modelos com funçôes risco monótonas.

Neste Capítulo apresentamos um estudo das propriedades da família de distribuição Weibull-exponenciada, bem como análises clássica e Bayesiana para tempos de vida com essa distribuição. O principal aporte deste capítulo é o uso de métodos Bayesianos para análise de dados de tempos de vida com distribuição Weibull-exponenciada.

\subsection{A distribuição Weibull-exponenciada}

A distribuição Weibull-exponenciada (WE) introduzida por Mudholkar et al. (1995), com parâmetros $\alpha, \theta$ e $\sigma$ para o tempo de vida $T$ tem função de densidade dada por

$$
f(t ; \alpha, \theta, \sigma)=\frac{\alpha \theta}{\sigma}\left[1-\exp \left(-(t / \sigma)^{\alpha}\right)\right]^{\theta-1} \times \exp \left(-(t / \sigma)^{\alpha}\right)(t / \sigma)^{\alpha-1}, \quad 0<t<\infty
$$

onde $\alpha>0, \theta>0$ são os parâmetros de forma e $\sigma>0$ é o parâmetro de escala. A densidade (2.1) pode ser usada para especificar a distribuição exponencial como caso particular quando, $\alpha=1$, e $\theta=1$ e a distribuição Weibull no caso em que $\theta=1$.

A função de distribuição de $T$, denotado por $F(t: \alpha, \theta, \sigma)$ para $t>0$ é dada por

$$
F(t ; \alpha, \theta, \sigma)=\left[1-\exp \left(-(t / \sigma)^{\alpha}\right)\right]^{\theta}
$$

\subsubsection{Momentos}

O $k$-ésimo momento da distribuição WE, para $k=1,2, \ldots$ é dado por

$$
\begin{aligned}
\mu_{k}^{\prime} & =E\left(T^{k}\right)=\int_{0}^{\infty} t^{k} f(t ; \alpha, \theta, \sigma) d t \\
& =\int_{0}^{\infty} t^{k} \frac{\alpha \theta}{\sigma}\left[1-\exp \left(-(t / \sigma)^{\alpha}\right)\right]^{\theta-1} \times \exp \left(-(t / \sigma)^{\alpha}\right)(t / \sigma)^{\alpha-1} d t \\
& =\theta \sigma^{k} \int_{0}^{\infty} y^{\frac{k}{\alpha}}(1-\exp (-y))^{(\theta-1)} \exp (-y) d y .
\end{aligned}
$$


Em geral os momentos são intratáveis analiticamente, porém, podem ser estudadas numericamente. Mas, quando $\theta$ é um número enteiro (2.3), é dado por

$$
\mu_{k}^{\prime}=\sigma^{k} \theta \Gamma\left(\frac{k}{\alpha}+1\right) \sum_{j=1}^{\theta-1}\left(\begin{array}{c}
\theta-1 \\
j
\end{array}\right) \frac{1}{(j+1)^{k / \alpha+1}} .
$$

Também, se $k / \alpha=r$ é um número énteiro e positivo, (2.3) se reduz a

$$
\mu_{k}^{\prime}=\sigma^{k} \theta(-1)^{r}\left[\frac{d^{r}}{d s^{r}} B(s, \theta)\right]_{s=1}
$$

onde $B(s, \theta)$ denota a função beta (veja, por exemplo, Abramovitz e Stegun, 1965).

\subsubsection{Função de sobrevivência e função risco}

A função de sobrevivência de $T$ que denotamos por $S(t ; \alpha, \theta, \sigma)=P(T \geq t)$ é dada por

$$
S(t ; \alpha, \theta, \sigma)=1-\left[1-\exp \left(-(t / \sigma)^{\alpha}\right)\right]^{\theta} .
$$

A função risco de $T$, definida por $h(t ; \alpha, \theta, \sigma)=f(t ; \alpha \theta, \sigma) / S(t ; \alpha, \theta, \sigma)$ é obtida apartir das expressões (2.1) e (2.6) e é dada por

$$
h(t ; \alpha, \theta, \sigma)=\frac{\alpha \theta\left[1-\exp \left(-(t / \sigma)^{\alpha}\right)\right]^{\theta-1} \exp \left(-(t / \sigma)^{\alpha}\right)(t / \sigma)^{\alpha-1}}{\sigma\left[1-\left(1-\exp \left(-(t / \sigma)^{\alpha}\right)\right)^{\theta}\right]} .
$$

A grande flexibilidade deste modelo para o ajuste de dados de tempo de vida é dada pelas diferentes formas que a função de risco (2.7) pode apresentar, ísto é,

(i) monótona crescente se $\alpha \geq 1$ e $\alpha \theta \geq 1$,

(ii) monótona decrescente se $\alpha \leq 1$ e $\alpha \theta \leq 1$,

(iii) forma de banheira se $\alpha>1$ e $\alpha \theta<1$ e

(iv) unimodal se $\alpha<1$ e $\alpha \sigma>1$.

As monotonicidades em (i) e (ii) são estritas exceto para a distribuição exponencial correspondente a $\alpha=\theta=1$.

A figura 2.1, mostra alguns casos especiais da função risco (2.7). 


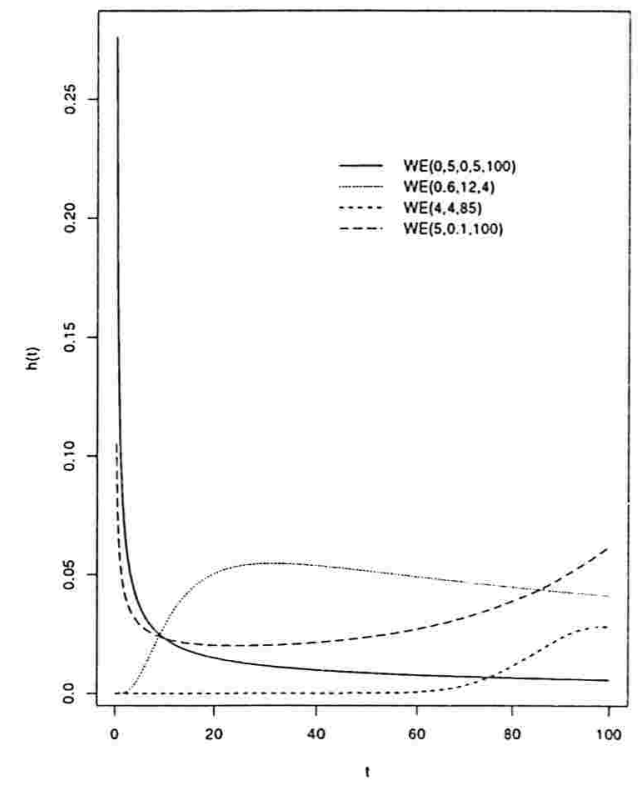

Figura 2.1: Alguma formas especiais da função risco da família de distribuição Weibullexponenciada

\subsection{Análise clássica do modelo Weibull-exponenciada}

Nesta seção apresentaremos o procedimento de inferência clássica considerando a distribuição Weibull-exponenciada para os tempos de vida. Consideramos o método de máxima verossimilhança para estimação dos parâmetros e métodos assintóticos para a construção de intervalos de confiança e teste aproximados.

\subsubsection{Estimação dos parâmetros por máxima verossimilhança}

Sejam $t_{1}, \ldots, t_{n}$ observações de uma amostra onde $t_{i}$ representa o tempo de vida ou tempo de censura de cada um dos $n$ indivíduos em estudo. Considerando que os tempos de vida têm distribuição Weibull-exponenciada com função de densidade dada em (2.1), a função de verossimilhança para $\alpha, \theta$ e $\sigma$ é dada por

$$
L(\alpha, \sigma, \theta)=\alpha^{r} \theta^{r} \sigma^{-r \alpha} \exp \left\{-\sum_{i \in F}\left(\frac{t_{i}}{\sigma}\right)^{\alpha}\right\} \prod_{i \in F} t_{i}^{\alpha-1}\left(1-\exp \left\{-\left(\frac{t_{i}}{\sigma}\right)^{\alpha}\right\}\right)^{\theta-1}
$$




$$
\times \prod_{i \in C}\left(1-\left(1-\exp \left\{-\left(\frac{t_{i}}{\sigma}\right)^{\alpha}\right\}\right)^{\theta}\right)
$$

onde $r$ é número de falhas observadas, $F$ denota o conjunto de observações não censuradas, $C$ denota o conjunto de observações censuradas.

Os estimadores de máxima verossimilhança (EMV) de $\alpha, \theta$ e $\sigma$ são valores que maximizam a função de verossimilhança em (2.8), o que é equivalente a maximizar o logaritmo da função de verossimilhança, isto é, que maximiza: $\ell(\alpha, \theta, \sigma)=\log (L(\alpha, \sigma, \theta))$, ou seja

$$
\begin{aligned}
\ell(\alpha \cdot \theta, \sigma)= & r \log \left(\frac{\alpha \theta}{\sigma}\right)+(\theta-1) \sum_{i \in F} \log \left(g\left(t_{i}\right)\right)-\sum_{i \in F}\left(\frac{t_{i}}{\sigma}\right)^{\alpha}+(\alpha-1) \sum_{i \in F} \log \left(\frac{t_{i}}{\sigma}\right) \\
& +\sum_{i \in C} \log \left(S\left(t_{i}\right)\right),
\end{aligned}
$$

onde $g\left(t_{i}\right)=g\left(t_{i} ; \alpha, \sigma\right)=1-\exp \left(-\left(t_{i} / \sigma\right)^{\alpha}\right)$ e $S\left(t_{i}\right)=S\left(t_{i}, \alpha, \theta, \sigma\right)$ é a função de sobrevivência dada em (2.6). Equivalentemente, os EMV resultam da solução do seguinte sistema de equações não lineares:

$$
\begin{aligned}
& \frac{\partial \ell}{\partial \alpha}=\frac{r}{\alpha}+(\theta-1) \sum_{i \in F} \frac{g_{\alpha}\left(t_{i}\right)}{g\left(t_{i}\right)}-\sum_{i \in F}\left(\frac{t_{i}}{\sigma}\right)^{\alpha} \log \left(\frac{t_{i}}{\sigma}\right)+\sum_{i \in F} \log \left(\frac{t_{i}}{\sigma}\right)+\sum_{i \in C} \frac{S_{\alpha}\left(t_{i}\right)}{S\left(t_{i}\right)}=0 \\
& \frac{\partial \ell}{\partial \theta}=\frac{r}{\theta}+\sum_{i \in F} \log \left(g\left(t_{i}\right)\right)+\sum_{i \in C} \frac{S_{\theta}\left(t_{i}\right)}{S\left(t_{i}\right)}=0 \\
& \frac{\partial \ell}{\partial \theta}=-\frac{r \alpha}{\sigma}+(\theta-1) \sum_{i \in F} \frac{g_{\beta}\left(t_{i}\right)}{g\left(t_{i}\right)}+\frac{\alpha}{\sigma} \sum_{i \in F}\left(\frac{t_{i}}{\sigma}\right)^{\alpha}+\sum_{i \in C} \frac{S_{\sigma}\left(t_{i}\right)}{S^{\prime}\left(t_{i}\right)}=0
\end{aligned}
$$

onde $g_{\alpha}\left(t_{i}\right)=\exp \left(-\left(\frac{t_{i}}{\sigma}\right)^{\alpha}\right)\left(\frac{t_{i}}{\sigma}\right)^{\alpha} \log \left(\frac{t_{i}}{\sigma}\right)^{2}, g_{\sigma}\left(t_{i}\right)=-\frac{\alpha}{\sigma} \exp \left(-\left(\frac{t_{i}}{\sigma}\right)^{\alpha}\right)\left(\frac{t_{i}}{\sigma}\right)^{\alpha}, S_{\alpha}\left(t_{i}\right)=-\theta g^{\theta-1}\left(t_{i}\right) g_{\alpha}\left(t_{i}\right)$, $S_{\theta}\left(t_{i}\right)=-g^{\theta}\left(t_{i}\right) \log \left(g\left(t_{i}\right)\right)$ e $S_{\sigma}\left(t_{i}\right)=-\theta g^{\theta-1}\left(t_{i}\right) g_{\sigma}\left(t_{i}\right)$.

Como não é possível encontrar uma forma analítica fechada para $\hat{\alpha}, \hat{\theta}$ e $\hat{\sigma}$ pode-se utilizar algum método numérico para resolver o sistema dado em (2.10)-(2.12). Um método mais utilizado é o método de Newton-Rapšnon. Esse método aparece como subrotina em alguns softwares, como por exemplo, no software Ox (veja Doornik, 1998). 


\subsubsection{Inferência sobre os parametros $\alpha, \theta$ e $\sigma$}

Inferência sobre os parâmetros são feitas usualmente através da normalidade assintótica dos estimadores de máxima verossimilhança (ver, por exemplo, Mudholkar et al. 1995). Assim, para amostras suficientemente grandes e sob certas condições de regularidade pode-se mostrar que

$$
i^{1 / 2}(\alpha, \theta, \sigma)\left[\left(\begin{array}{c}
\hat{\alpha} \\
\hat{\theta} \\
\hat{\sigma}
\end{array}\right)-\left(\begin{array}{c}
\alpha \\
\theta \\
\sigma
\end{array}\right)\right] \stackrel{D}{\longrightarrow} N\left(0, I_{3}\right),
$$

onde $i(\alpha, \theta, \sigma)^{1 / 2}$ é a matriz raiz quadrada da matriz de informação de Fisher e $I_{3}$ é a matriz identidade de ordem $3 \times 3$. A distribuição assintótica dos EMV segue sendo válido se a matriz de informação de Fisher é substituida pela matriz de informação observada dada por

$$
I(\hat{\alpha}, \hat{\theta}, \hat{\sigma})=\left(\begin{array}{ccc}
-\frac{\partial^{2} \ell}{\partial \alpha^{2}} & -\frac{\partial^{2} \ell}{\partial \alpha \partial \theta} & -\frac{\partial^{2} \ell}{\partial \alpha \partial \sigma} \\
& -\frac{\partial^{2} \ell}{\partial \theta^{2}} & -\frac{\partial^{2} \ell}{\partial \theta \partial \sigma} \\
\text { simétrica } & & -\frac{\partial^{2} \ell}{\partial \sigma^{2}}
\end{array}\right)_{\left.\right|_{(\alpha, \theta, \sigma)=\{\dot{\alpha}, \hat{\theta}, \hat{\sigma})}} .
$$

Considerando a normalidade assintótica dos estimadores de máxima verossimilhaça $\hat{\alpha}, \hat{\theta}$ e $\hat{\sigma}$ podem-se calcular os intervalos de confiança para os parâmetros $\alpha, \theta$ e $\sigma$ e para a função de sobrevivência $S\left(t_{0}\right)$ para dado $t_{0}$.

Representando a inversa da matriz de informação observada por

$$
I^{-1}(\hat{\alpha}, \hat{\theta}, \hat{\sigma})=\left(\begin{array}{lll}
b_{11} & b_{12} & b_{13} \\
b_{21} & b_{22} & b_{23} \\
b_{31} & b_{32} & b_{33}
\end{array}\right)
$$

e considerando um nível de confiança de $100(1-\alpha) \%$, os intervalos de confiança aproximados para $\alpha, \theta$ e $\sigma$ são dados por:

$$
\begin{aligned}
i c(\alpha) & =\left[\hat{\alpha}-z_{\alpha / 2}\left(b_{11}\right)^{1 / 2} ; \hat{\alpha}+z_{\alpha / 2}\left(b_{11}\right)^{1 / 2}\right], \\
i c(\theta) & =\left[\hat{\theta}-z_{\alpha / 2}\left(b_{22}\right)^{1 / 2} ; \hat{\theta}+z_{\alpha / 2}\left(b_{22}\right)^{1 / 2}\right], \\
i c(\sigma) & =\left[\hat{\sigma}-z_{\alpha / 2}\left(b_{11}\right)^{1 / 2} ; \hat{\sigma}+z_{\alpha / 2}\left(b_{22}\right)^{1 / 2}\right],
\end{aligned}
$$


onde $z_{\alpha / 2}$ é o percentil correspondente da distribuição normal padronizada e $b_{i i}$ é o $i$-ésimo elemento da diagonal da inversa da matriz de informação observada $I$ avaliada nos EMV.

\subsubsection{Teste de bondade de ajuste}

O problema do teste de bondade de ajuste do modelo Weibull contra a classe de alternativas irrestrito é complexo. Contudo, restringindo as alternativas para a família Weibullexponenciada podemos usar a estatística da razão de verossimilhanças (veja Rao,1973) para testar a adequabilidade do submodelo Weibull. A hipótese nula, $H_{01}: \theta=1$ cor-

respondente ao submodelo Weibull e $H_{02}:\{\theta=1, \alpha=1\}$ correspondente ao submodelo exponencial (do modelo Weibull-exponenciada). Se uma destas hipóteses ou ambas são rejeitadas na classe da família Weibull-exponenciada então os submodelos são questionáveis. A estatística da razão de verossimilhanças para testar $H_{0 i}(i=1,2)$ são dadas por:

$$
\Lambda_{i}=\sup _{R_{0 i}}\{L(\alpha, \theta, \sigma)\} / \sup _{R}\{L(\alpha, \theta, \sigma)\}, i=1,2,
$$

onde $R_{0 i}$ é o espaço paramétrico correspondente a $H_{0 i}, i=1,2$, e $R$ é o espaço paramétrico irrestrito.

Em termos dos EMVs, a estatística da razão de verossimilhanças para testar a adequabilidade do modelo Weibull, isto é, $H_{01}: \theta=1$ contra $H_{11}: \theta \neq 1$, se reduz a

$$
\Lambda_{1}=L\left(\hat{\alpha}_{w}, \theta=1, \hat{\sigma}_{w}\right) / L(\tilde{\alpha}, \tilde{\theta}, \tilde{\sigma})
$$

onde $\hat{\alpha}_{w}$ e $\hat{\sigma}_{w}$ são EMV dos parâmetros do modelo Weibull e $\tilde{\alpha}, \tilde{\theta}$ e $\tilde{\sigma}$ são os EMV dos parâmetros do modelo Weibull-exponenciada. Sob a hipótese $H_{01},-2 \log \left(\Lambda_{1}\right)$ tem distribuição aproximada qui-quadrado com $u m$ grau de liberdade para tamanhos amostrais suficientemente grandes (veja Rao, 1973). Similarmente para testar a adequabilidade do modelo exponencial a estatística da razão de verossimilhança é dada por

$$
\Lambda_{2}=L\left(\alpha=1, \theta=1, \hat{\sigma}_{e}\right) / L(\tilde{\alpha}, \tilde{\theta}, \tilde{\sigma})
$$


onde $\hat{\sigma}_{e}$ é o EMV do parâmetro no modelo exponencial. Sob a hipótese $H_{02}:\{\theta=1, \alpha=$ $1\},-2 \log \left(\Lambda_{2}\right)$ tem distribuição aproximada qui-quadrado com 2 graus de liberdade para tamanhos amostrais suficientemente grandes.

\subsection{Análise Bayesiana do modelo Weibull-exponenciada}

Na seção 2.3 foi considerada uma análise clássica do modelo Weibull-exponenciada para os tempos de falha, baseada em resultados assintóticos. Para amostras de tamanhos moderados ou pequenos esses resultados podem não ser confiáveis, tornando-se assim um problema sério uma vez que em geral, trabalha-se com amostras não muito grandes. Um procedimento alternativo que pode contornar esse problema é através da utilização de métodos Bayesianos. O uso de métodos Bayesianos, além de serem uma alternativa de análise, permitem ainda a incorporação de conhecimento a priori através de uma densidade a priori que seja informativa. Nesta seção apresenta-se uma análise Bayesiana do modelo Weibull-exponenciada usando o método de simulação via cadeias de Markov (veja, Gelfand e Smith, 1990).

\subsubsection{Densidade conjunta a posteriori para os parâmetros do modelo Weibull-exponenciada}

Para representar o grau de conhecimento sobre os parâmetros do modelo Weibull-exponenciada (2.1), consideramos para $\alpha, \theta$ e $\sigma$ as seguintes distribuições a priori:

$$
\begin{aligned}
\alpha & \sim \Gamma\left(a_{1}, b_{1}\right), \\
\theta & \sim \Gamma\left(a_{2}, b_{2}\right), \\
\sigma & \sim \Gamma\left(a_{3}, b_{3}\right),
\end{aligned}
$$

onde $\Gamma\left(a_{i}, b_{i}\right)$ denota a distribuição gama com média $a_{i} / b_{i}$ e variância $a_{i} / b_{i}^{2}$, e $a_{i}$ e $b_{i}$, $i=1,2,3$ são constantes conhecidas baseadas na informação a priori do especialista e da 
análise preliminar dos dados.

Assumindo independência entre os parâmetros a densidade a priori conjunta de $\alpha, \theta$ e $\sigma$ é dada por:

$$
\pi(\alpha, \theta, \sigma) \propto \alpha^{a_{1}-1} \theta^{a_{2}-1} \sigma^{a_{3}-1} \exp \left\{-\alpha b_{1}-\theta b_{2}-\sigma b_{3}\right\}
$$

onde $\alpha>0, \theta>0$ e $\sigma>0$.

Observa-se que no caso em que $a_{i}=b_{i}=0,(i=1,2,3)$ a densidade a priori conjunta de $\alpha, \theta$ e $\sigma$ dada em (2.17) é imprópria, ou seja,

$$
\pi(\alpha, \theta, \sigma) \propto \frac{1}{\alpha \theta \sigma} .
$$

Combinando (2.8)-(2.17) e o teorema de Bayes pode-se mostrar que a densidade conjunta a posteriori de $\alpha, \theta$ e $\sigma$ é dada por

$$
\begin{aligned}
\pi(\alpha, \theta, \sigma \mid D) & \propto \alpha^{n+a_{1}-1} \theta^{n+n_{2}-1} \sigma^{a_{3}-n \alpha-1} \exp \left\{-\sum_{i \in F}\left(\frac{t_{i}}{\sigma}\right)^{\alpha}-b_{1} \alpha-b_{2} \theta-b_{3} \sigma\right\} \\
& \times \prod_{i \in F} t_{i}^{\alpha-1}\left(1-\exp \left\{\left(\frac{t_{i}}{\sigma}\right)^{\alpha}\right\}\right)^{\theta-1} \prod_{i \in C}\left(1-\left(1-\exp \left\{\left(\frac{t_{i}}{\sigma}\right)^{\alpha}\right\}\right)^{\theta}\right),
\end{aligned}
$$

onde $\alpha>0, \theta>0, \sigma>0$ e $D$ denota o conjunto de dados observados.

Notamos que a densidade a posteriori conjunta (2.18) não é uma densidade padrão, portanto só podemos avaliar as densidades a posteriori marginais por meio de métodos de aproximação, tais como o método de Laplace (Tierney , Kass e Kadane, 1986) ou usando métodos de simulação de Monte Carlo via Cadeias de Markov, tais como Gibbs Sampling (Casela e George, 1992) e Metropolis-Hasting (Chib e Greenberg, 1995). Nesta análise consideramos os métodos de simulação por serem de fácil implementação computacional.

Para obtermos uma amostra a posteriori das densidades marginais $\alpha, \sigma$ e $\theta$, fazemos uso do algoritmo de Gibbs Sampling que baseia-se em sucessivas gerações das distribuições condicionais a posteriori de $\pi(\alpha \mid \theta, \sigma, D), \pi(\theta \mid \alpha, \sigma, D)$ e $\pi(\sigma \mid \alpha, \theta, D)$.

Da densidade a posteriori conjunt dada em (2.18) pode-se mostrar que as densidades 
condicionais a posteriori para o algoritmo de Gibbs são dadas por

$$
\begin{aligned}
\pi(\alpha \mid \theta, \sigma, D) & \propto \alpha^{n+a_{1}-1} \sigma^{-\alpha n} \exp \left\{-\sum_{i \in F}\left(\frac{t_{i}}{\sigma}\right)^{\alpha}-b_{1} \alpha+\alpha \sum_{i \in F} \log \left(t_{i}\right)+A(\alpha, \sigma, \theta)\right\} \\
\pi(\theta \mid \alpha, \sigma, D) & \propto \theta^{a_{2}+n-1} \exp \left\{-\theta b_{2}+A_{1}(\alpha, \theta, \sigma)\right\} \\
\pi(\sigma \mid \alpha, \theta, D) & \propto \sigma^{a_{3}-n \alpha-1} \exp \left\{-\sum_{i \in F}\left(\frac{t_{i}}{\sigma}\right)^{\alpha}-b_{3} \theta+A(\alpha, \theta, \sigma)\right\}
\end{aligned}
$$

onde

$$
\left.A(\alpha, \theta, \sigma)=(\theta-1) \sum_{i \in F} \log \left(1-\exp \left\{-\left(\frac{t_{i}}{\sigma}\right)^{\alpha}\right\}\right)\right)+\sum_{i \in C} \log \left(1-\left(1-\exp \left\{-\left(\frac{t_{i}}{\sigma}\right)^{\alpha}\right\}\right)^{\theta}\right)
$$

e

$$
\left.A_{1}(\alpha, \theta, \sigma)=\theta \sum_{i \in F} \log \left(1-\exp \left\{-\left(\frac{t_{i}}{\sigma}\right)^{\alpha}\right\}\right)\right)+\sum_{i \in C} \log \left(1-\left(1-\exp \left\{-\left(\frac{t_{i}}{\sigma}\right)^{\alpha}\right\}\right)^{\theta} .\right.
$$

Observe que as densidades dadas em (2.19) não são conhecidas de modo que faremos uso do algoritmo de Metropolis-Hasting para gerar as variáveis $\alpha, \theta$ e $\sigma$.

\subsubsection{Estimador de Bayes da função de sobrevivência e função risco}

Considerando as amostras Gibbs geradas em (2.19) de $\alpha, \theta$ e $\sigma$, pode-se obter estimativas de Monte Carlo para os momentos de interesse. Um caso especial deste é dado pela função de sobrevivência (2.6) para um valor do tempo $t_{0}$ especificado. Um estimador de Bayes para $S\left(t_{0}\right)$ com respeito à função perda quadrática é dada por

$$
E\left[S\left(t_{0} \mid D\right)\right]=\iiint\left(1-\left[1-\exp \left(-\left(t_{0} / \sigma\right)^{\alpha}\right)\right]^{\theta}\right) \pi(\alpha, \theta, \sigma \mid D) d \alpha \theta \sigma
$$

a qual pode-se aproximar por sua estimativa de Monte Carlo por

$$
\hat{S}\left(t_{0}\right)=\frac{2}{R S} \sum_{r=1}^{R} \sum_{s=\frac{S}{2}+1}^{S}\left[1-\left[1-\exp \left(-\left(\frac{t_{0}}{\sigma^{(r, s)}}\right)^{\alpha^{(r, s)}}\right)\right]^{\theta^{(r, s)}}\right] .
$$

onde $R$ e $S$ são respectivamente, número total de iterações e simulações do amostrador de Gibbs. Similarmente, uma estimativa da função risco (2.7) para um valor do tempo 
$t_{0}$ especificado com respeito à função de perda quadrática é dada por $E\left[h\left(t_{0}\right) \mid D\right]$. Uma estimativa de Monte Carlo é dada por

$$
\hat{h}\left(t_{0}\right)=\frac{2}{R S} \sum_{r=1}^{R} \sum_{s=\frac{S}{2}+1}^{S} \frac{\alpha^{(r, s)} \theta^{(r, s)}\left[1-\exp \left(-\left(\frac{t_{0}}{\sigma^{(r, s)}}\right)\right)\right]^{\theta^{(r, s)}-1} \exp \left(-\left(\frac{t_{0}}{\sigma^{(r, s)}}\right)^{\alpha^{(r, s)}}\right)\left(\frac{t_{0}}{\sigma^{(r, s)}}\right)^{\alpha^{(r, s)}-1}}{\sigma^{(r, s)}\left[1-\left(1-\exp \left(-\left(\frac{t_{0}}{\sigma^{(r, s)}}\right)^{\alpha^{(r, s)}}\right)^{\theta^{(r, s)}}\right]\right.},
$$

onde $\alpha^{(r, s)}, \sigma^{(r, s)}$ e $\theta^{(r, s)}$ denotam as amostras de $\alpha, \sigma$ e $\theta$ na $r$-ésima iteração e $s$-ésima réplica.

\subsubsection{Densidade conjunta a posteriori dos parâmetros do mode- lo Weibull}

Consideramos as seguintes densidades a priori para $\alpha$ e $\sigma$ para os parâmetros da distribuição de Weibull:

$$
\begin{aligned}
& \alpha \sim \Gamma\left(a_{1}, b_{1}\right), \quad a_{1} \text { e } b_{1} \text { são conhecidos } \\
& \sigma \sim \Gamma\left(a_{2}, b_{2}\right), a_{2} \text { e } b_{1} \text { são conhecidos, }
\end{aligned}
$$

onde $\Gamma\left(a_{i}, b_{i}\right)$ denota a distribuição Gama com média $a_{i} / b_{i}, i=1,2$.

Assumindo independência entre os parâmetros da distribuição Weibull e considerando a função de verossimilhança de (2.S) $\operatorname{com} \theta=1$, pode-se mostrar que a densidade conjunta a posteriori de $\alpha$ e $\sigma$ é dada por

$$
\pi(\alpha, \sigma \mid D) \propto \alpha^{a_{1}+r-1} \sigma^{a_{2}-r \alpha-1} \exp \left\{-\sum_{i=1}^{n}\left(\frac{t_{i}}{\sigma}\right)^{\alpha}-(\alpha-1) \sum_{i \in F} \log \left(t_{i}\right)-\alpha b_{1}-\sigma b_{2}\right\},
$$

onde $\alpha>0, \sigma>0$. Notemos que a distribuição a posteriori dada em (2.24) não tem uma forma padrão conhecida pelo que faremos uso do método MCMC.

Para obtermos uma amostra a posteriori das densidades marginais de $\alpha$ e $\sigma$, exploramos o uso do método do amostrador de Gibbs que baseia-se em sucesivas gerações das distribuições marginais a posteriori $\pi(\alpha \mid \sigma, D)$ e $\pi(\sigma \mid \alpha, D)$. 
De (2.24) pode-se mostrar que as densidades a posteriori condicionais para o algoritmo de Gibbs são dadas por

$$
\begin{aligned}
& \pi(\alpha \mid \sigma, D) \propto \alpha^{r+a_{1}-1} \sigma^{-r \alpha} \exp \left\{-\sum_{i=1}^{n}\left(\frac{t_{i}}{\sigma}\right)^{\alpha}-b_{1} \alpha+\alpha \sum_{i \in F} \log \left(t_{i}\right)\right\} \\
& \pi(\sigma \mid \alpha, D) \propto \sigma^{a_{1}-r \alpha-1} \exp \left\{-\sum_{i=1}^{n}\left(\frac{t_{i}}{\sigma}\right)^{\alpha}-b_{2} \sigma\right\} .
\end{aligned}
$$

Observe que as densidades (2.25) não tem uma forma padrão conhecida e portanto deve-se usar o algoritmo de Metropolis-Hasting para gerar amostras de $\alpha$ e $\sigma$.

\subsection{Exemplo de aplicação}

Nesta seção ilustramos a metodologia apresentadas nas seções anteriores, através da análise do conjunto de dados analizado por Efron (1988) com reanalize em Mudholkar et al. (1995).

Na tabela 2.1, apresenta-se os tempos de sobrevivência em dias de 51 pacientes de cancer na região da cabeça-pescoço.

Tabela 2.1: Tempos de Sobrevivência de pacientes de cancer, com + indicando censura

\begin{tabular}{lllllllllll}
\hline \hline 7 & 34 & 42 & 63 & 64 & $74^{+}$ & 83 & 84 & 91 & 108 & 112 \\
129 & 133 & 133 & 139 & 140 & 140 & 146 & 149 & 154 & 157 & 160 \\
160 & 165 & 173 & 176 & $185^{+}$ & 218 & 225 & 241 & 248 & 273 & 277 \\
$279^{+}$ & 297 & $319^{+}$ & 405 & 417 & 420 & 440 & 523 & $523^{+}$ & 583 & 594 \\
1101 & $1116^{+}$ & 1146 & $1226^{+}$ & $1349^{+}$ & $1412^{+}$ & 1417 & & & & \\
\hline \hline
\end{tabular}

\section{Análise clássica}

Considerando a log-verossimilhança dada em (2.9) temos que o estimador de máxima verossimilhança do parâmetro $\boldsymbol{\eta}=(\alpha, \theta, \sigma)$ é obtido pela resolução do sistema de equações não linerares dada em (2.10). Isto é feito através da rotina de Newton-Rapson do pacote Ox. 
As estimativas de máxima verossimilhança são dadas por

$$
\hat{\alpha}=0,2943(0,1390 \tau), \hat{\theta}=18,036(27,410), \quad \hat{\sigma}=0,14404(0,4995) .
$$

Cada valor entre parênteses ao lado das estimativas representa a raiz quadrada do correspondente elemento da diagonal da matriz $I^{-1}(\hat{\alpha}, \hat{\theta}, \hat{\sigma})$, onde $I(\alpha, \theta, \sigma)$ é a matriz de informação observada. Se admitimos a normalidade assintótica para os estimadores de máxima verossimilhança, $I^{-1}(\hat{a}, \hat{\theta}, \hat{\sigma})$ corresponde à estimativa da matriz de covariâncias assintótica, e pode ser utilizada para fazer inferências sobres os parâmetros.

A estatística da razão de verosimilhanças para testar $H_{01}: \theta=1$ dada em (2.15) é $\Lambda_{1}=7,7$, e indica a não aceitação do modelo de Weibull a um nível de significância 0,05 $\left(\chi_{(1,0,05)}^{2}=3,8441\right)$.

\section{Análise Bayesiana}

Para analisarmos os dados da tabela 2.1, desde uma perpectiva Bayesiana consideramos a densidade a priori para $\alpha, \theta$. e $\sigma$ dada em (2.17) com $a_{1}=8,66, b_{1}=29,43, a_{2}=31,27$ $, b_{2}=1,734, a_{3}=2,5614$ e $b_{3}=17,78$ (a escolha desses valores para os parâmetros da densidade a priori, foram baseadas numa opinião experinte combinada com uma análise preliminar dos dados). A partir da densidades condicionais (2.19), geramos para cada um dos parâmetros do modelo Weibull-exponenciada 10 cadeias separadas de Gibbs cada uma com 5.000 iterações, e devido ao fato de todas as densidades condicionais marginais não apresentarem uma forma padrão conhecida utilizamos então o algoritmo de MetropolisHasting. Com o objetivo de diminuir o efeito de pontos iniciais deprezam-se as 2.500 primeiras iterações e a partir daí escolhem-se as iterações de 25 em 25 , ou seja, para cada parâmetro considera-se a 2.525 - ésima, . ., 5.000 - ésima iteraçoes; de modo que as 10 cadeias fornecem uma amostra Gibbs de tamanho 1000 para cada um dos parâmetros. A convergência das amostras geradas pelo algoritmo de Gibbs com Metropolis-Hasting foi monitorada utilizando o método proposto por Gelman e Rubin (1992) o qual se baseia na técnica de analise de variância. 
$\mathrm{Na}$ tabela 2.2, apresenta-se um resumo das distribuições a posteriori marginais aproximadas e as estimativas dos fatores de redução de escala potencial $\hat{R}$ (ver por exemplo, Gelman e Rubin, 1992). Observamos na tabela 2.2, que $\sqrt{\hat{R}}$ são bem menores que 1.1, o que nos indica que o algoritmo de Gibbs com Metropolis-Hasting gera para cada parâmetro amostras que convergem em distribuição para as distribuições condicionais marginais (2.19).

Tabela 2.2: Sumário a posteriori dos parâmetros do modelo Weibull-exponenciada

\begin{tabular}{|llllcc|}
\hline \hline & Média & Mediana & D.P. & Intervalo de credibilidade $95 \%$ & $\hat{R}$ \\
\hline$\alpha$ & 0,3207 & 0,3197 & 0,0373 & $(0,2515 ; 0,3746)$ & 1,000271 \\
$\theta$ & 18,3323 & 18,0802 & 4,1213 & $(11,1911 ; 24,4389)$ & 1,00014 \\
$\sigma$ & 0,16057 & 0,1518 & 0,0703 & $(0,0550 ; 0,2705)$ & 1,001939 \\
\hline \hline
\end{tabular}

Da tabela 2.2, note que os desvios padrões das estimativas obtidas pelo método MCMC são bem menores que os desvios padrões obtidos invertendo-se a matriz de informação observada. As médias a posteriori são próximas dos estimadores de máxima verossimilhança.

Na figura 2.2, representa-se as densidades a posteriori marginais aproximadas consideranda os 1.000 pontos amostrais.

Com a finalidade de escolher entre os modelos Weibull e Weibull-exponenciada desde uma perspectiva Bayesiana ( $H_{01}: \theta=1$ ), utiliza-se a técnica das densidades preditivas condicionadas ordenadas (CPO) conforme descrito no capítulo 1. Para isso, consideramos a partir das estimativas dos CPOs a estimativa da verossimilhança preditiva para definir o pseudo-fator de Bayes. A estimativa da verossimilhança preditiva sob o modelo de Weibull-exponenciada com as 1.000 amostras de Gibbs resultou em $\hat{f}\left(\underline{t} \mid M_{1}\right)=7,584255 \times$ $10^{-68}$, e para o modelo de Weibull essa verossimilhança resultou em $\hat{f}\left(\underline{t} \mid M_{0}\right)=3,21158 \times$ $10^{-78}$. O pseudo-fator de Bayes do modelo Weibull com respeito ao modelo Weibullexponenciada resultou em $P S F B_{01}=4,23398 \times 10^{-11}<1$ o qual indica que o modelo 


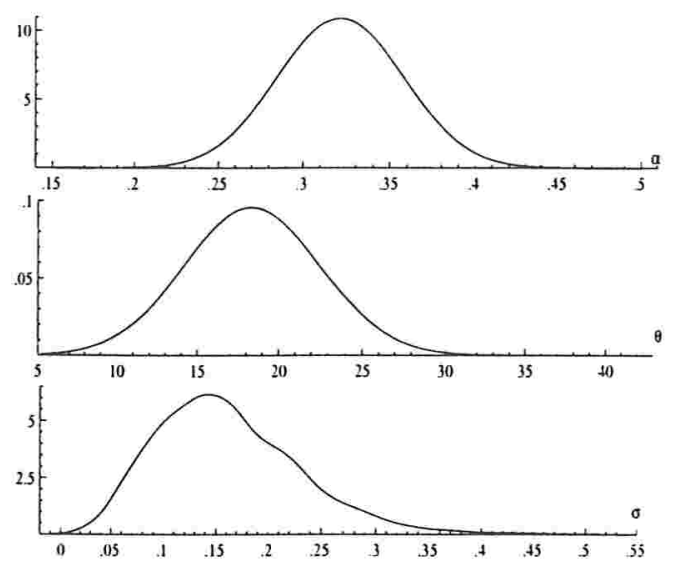

Figura 2.2: Densidades marginais a posteriori approximadas para os parâmetros do modelo Weibull-exponenciado, $\alpha, \theta$ e $\sigma$

Weibull-exponenciada é mais adequado para ajustar os dados da tabela 2.1 e esse resultado coincide com o resultado do teste de hipóteses clássica. Contudo o enfoque Bayesiano apresenta uma indicação muito mais forte do que o enfoque clássico que o modelo Weibullexponenciada é o mais adequado para o ajuste dos dados da tabela 2.1.

Na figura 2.3, plotamos o $\log$ a razão dos CPOs dos modelos Weibull e Weibullexponenciada com respeito ao número de observações. Valores positivos do log da razão dos CPO indica a preferência do primeiro modelo. Notamos a partir da figura 2.3, o gráfico indica que 44 das 51 observações suportam ao modelo Weibull-exponenciada sobre o modelo Weibull. Esse método gráfico também confirma os resultados obtidos pelos métodos clássico e Bayesiano.

Na figura 2.4, temos densidade preditiva para um tempo de vida futuro, $t$, dada as observações $D$ aproximada pelo método de Monte Carlo com as 1.000 amostras Gibbs dos parâmetros do modelo Weibull-exponenciada.

Na figura 2.5, representamos as função de sobrevivência a posteriori dada em (2.20) 


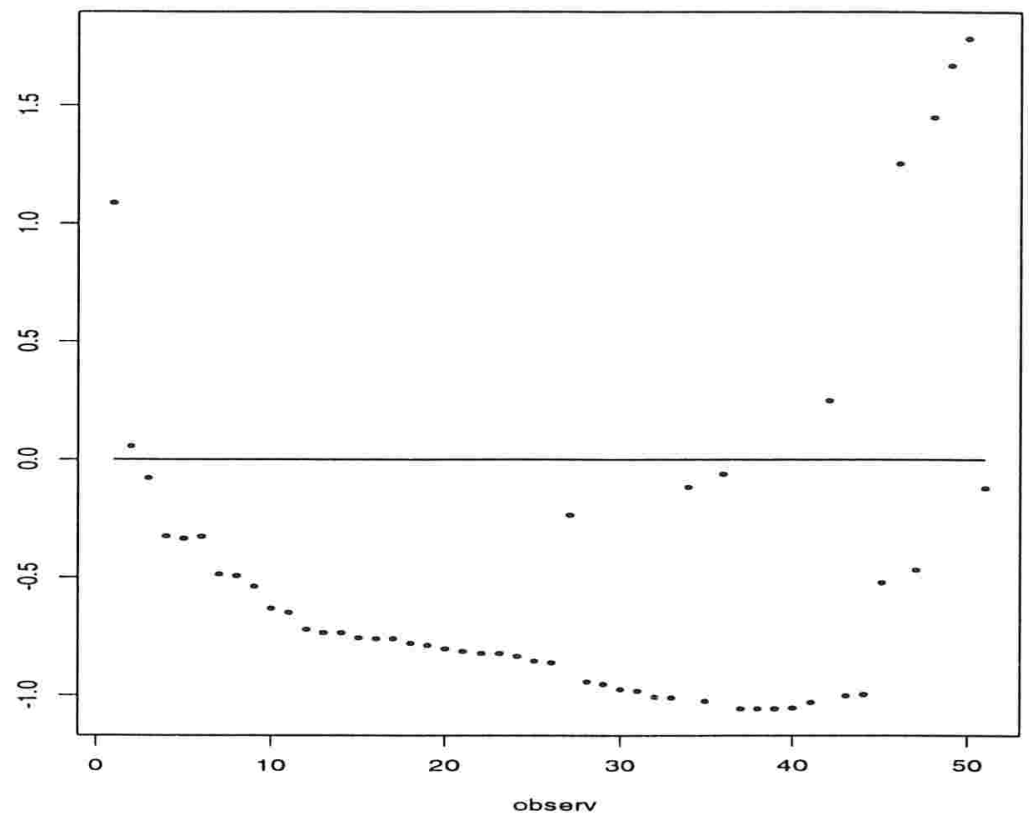

Figura 2.3: Gráfico do log da razão dos CPOs do modelo Weibull e o modelo Weibullexponenciada

e a função risco a posteriori dada em (2.21), aproximadas pelo método de Monte Carlo com as 1.000 amostras Gibbs. 


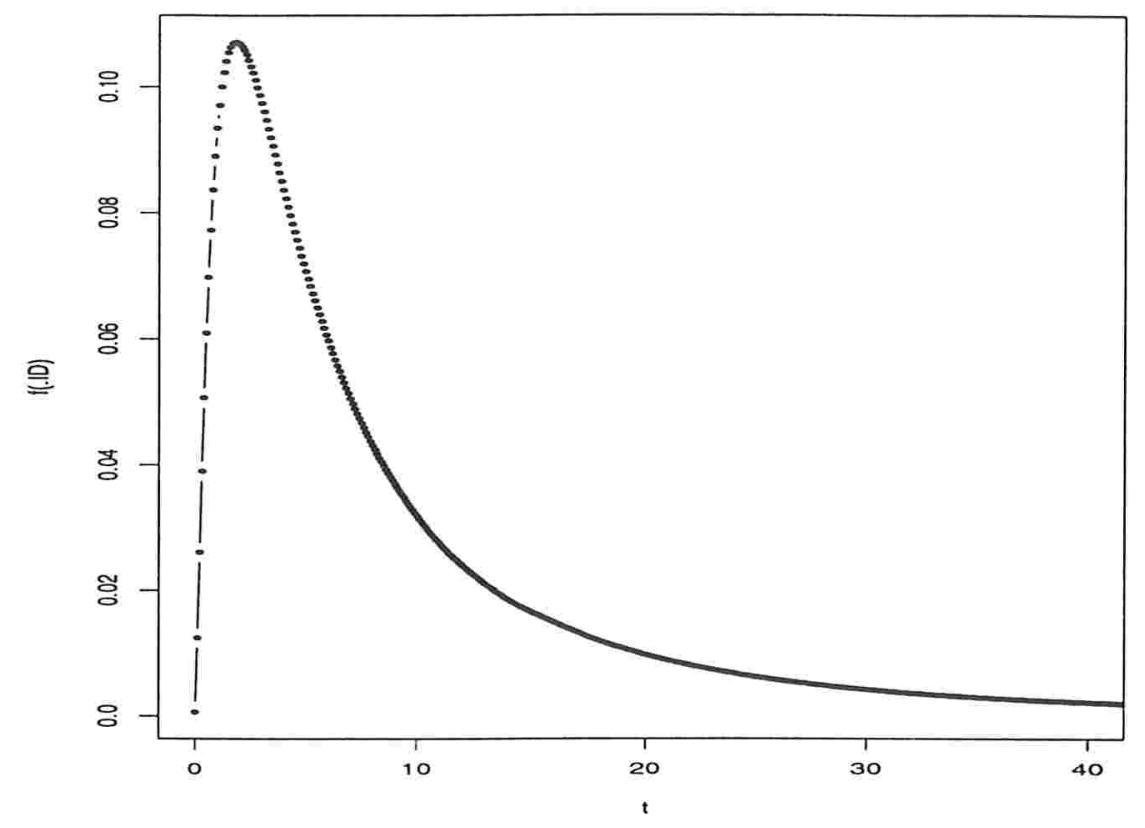

Figura 2.4: Densidade preditiva para tempo de vida futuro no modelo Weibullexponenciada
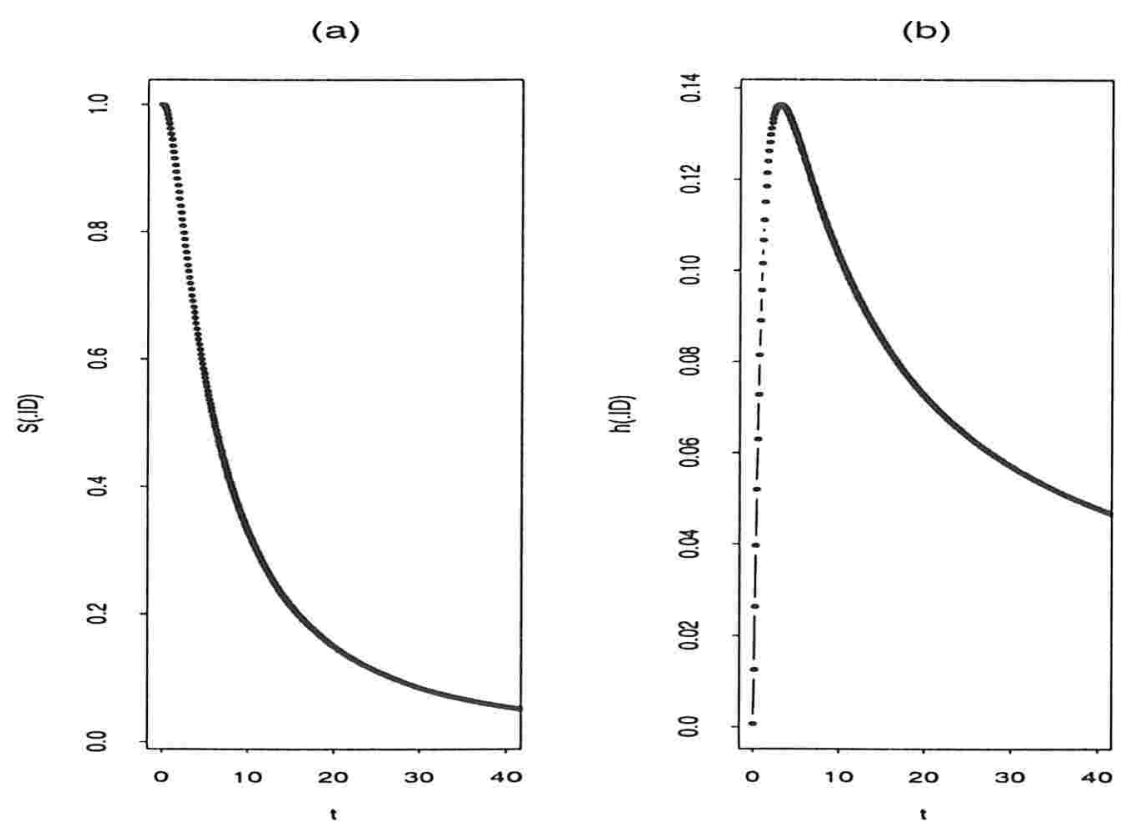

Figura 2.5: As funções : (a) sobrevivência e (b) risco a posteriori para o modelo Weibullexponenciada 


\section{Capítulo 3}

\section{Modelos de regressão}

\section{Weibull-exponenciada}

\subsection{Introdução}

Em muitas situações práticas temos que o tempo de vida é influenciado por uma ou mais variáveis regressoras. Por exemplo, o tempo de vida de um determinado isolante é naturalmente afetado pelo nível de voltagem a que ele é sujeito e o tempo de sobrevivência de um paciente pode depender da idade, da quantidade de cigarros que ele fuma por dia, e de uma série de outros fatores. Uma maneira de determinar o relacionamento entre o tempo de vida e a covariável $\mathrm{x}=\left(x_{i}, \ldots, x_{p}\right)$ é através de um modelo de regressão. Duas classes importantes de modelos de regressão são: modelos de riscos proporcionais para $T$ e modelos de locação escala para o $\log T$. Abordaremos aqui apenas a segunda classe. Um descrição detalhada sobre modelos de riscos proporcionais podem ser obtidas em Cox e Oakes (1989), Kalbleisch e Prentice (1980), entre outros. Neste Capítulo, consideramos que o parâmetro de escala $\sigma$ da distribuição Weibull-exponencianda dada em (2.1) depende de uma matriz de variáveis explicativas $\mathrm{x}$. 


\subsection{O Modelo log-Weibull-exponenciada}

Considerando a transformação da variável aleatória $Y=\log T$ e a reparametrização $\sigma=$ $\exp (\mu)$ e $\alpha=1 / \delta$, temos em (2.1), que a função de densidade de probablidades de $Y$ pode ser escrita como

$$
f(y)=\frac{\theta}{\delta}\left[1-\exp \left\{-\exp \left(\frac{y-\mu}{\delta}\right)\right\}\right]^{\theta-1} \exp \left\{\left(\frac{y-\mu}{\delta}\right)-\exp \left\{\left(\frac{y-\mu}{\delta}\right)\right\}\right\},
$$

onde $-\infty<y<\infty, \delta>0, \theta>0$ e $-\infty<\mu<\infty$. Observe que se $\theta=1$, temos a densidade valor extremo para $\mathrm{Y}$ com parâmetro de locação $\mu$ e parâmetro de escala $\delta$.

De (3.1), pode-se representar o modelo log-linear dado por

$$
Y=\mu+\delta Z
$$

onde $Z$ tem densidade dada por

$$
f(z)=\theta[1-\exp \{-\exp (z)\}]^{\theta-1} \exp \{z-\exp (z)\}, \quad-\infty<z<\infty,
$$

onde $\theta>0$. Observe que quando $\theta=1$, (3.3) é a densidade valor extremo padrão (ver por exemplo Lawless, 1982)

A figura 3.1, mostra algumas representações da densidade (3.3) para alguns valores dos parâmtros $(\theta=0,4 ; 1 ; 10 ; 100)$.

A função de sobrevivência é dada por

$$
S(y ; \mu, \theta, \delta)=1-\left\{1-\exp \left(-\exp \left(\frac{y-\mu}{\delta}\right)\right)\right\}^{\theta} .
$$

\subsection{O Modelo de regressão Weibul-exponenciada}

Consideremos agora o modelo de regressão baseado na distribuição log-Weibull-exponenciada, dada em (3.1). Assumimos uma relação linear entre o log do tempo de vida, denotado 

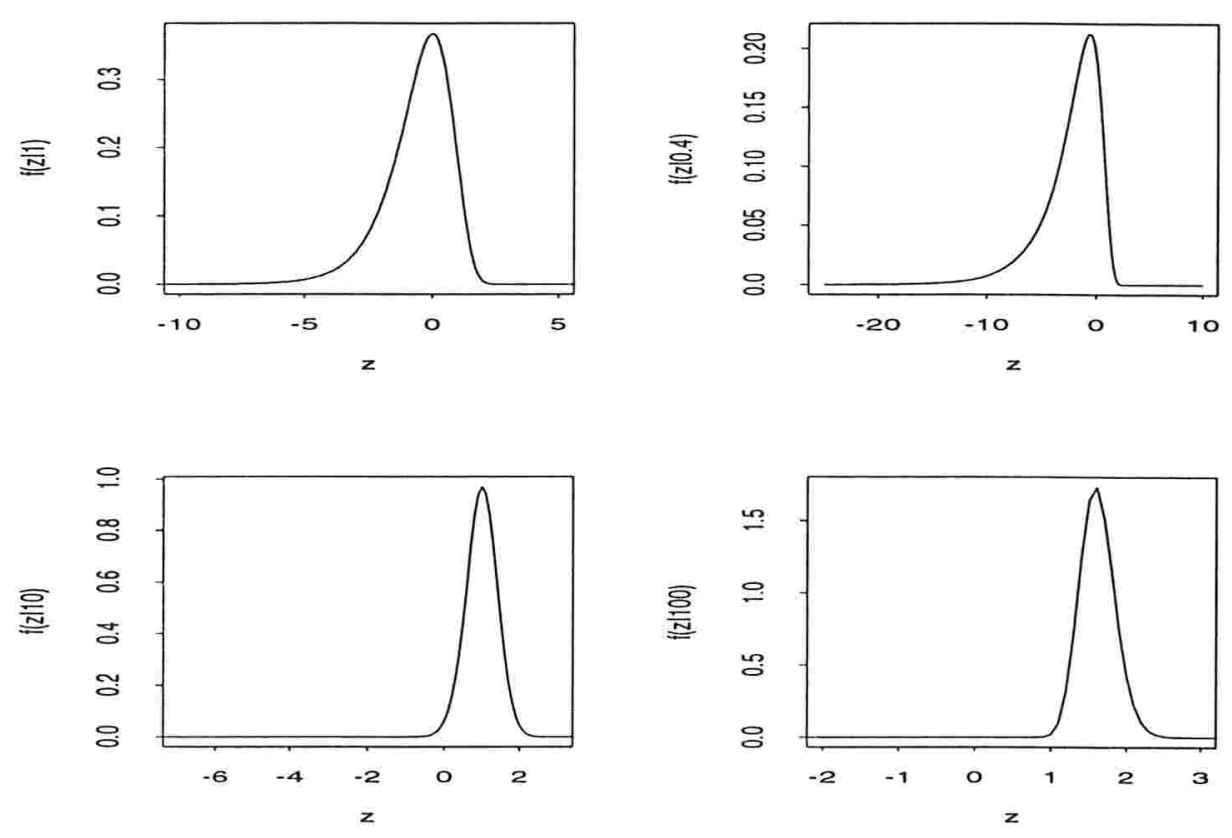

Figura 3.1: Algumas formas da densidade dada em (3.3).

por $Y$ e o vetor de covariáveis $\mathbf{x}=\left(x_{1}, \ldots, x_{p}\right)$. $Y$ dado $\mathrm{x}$ tem distribuição log-Weibulexponenciada e a relação linear entre $\mathrm{x}$ e $Y$ pode ser representada da forma,

$$
Y=\mathbf{x} \boldsymbol{\beta}+\delta Z
$$

onde $\boldsymbol{\beta}=\left(\beta_{1}, \ldots, 3_{p}\right)^{\prime}$ e $\delta>0$, são parâmetros desconhecidos, e $Z$ tem distribuição dada em (3.3).

A função de sobrevivência de $Y$ dado $\mathrm{x}$, analogamente a (3.4), é representada por

$$
S(y ; \theta, \delta, \boldsymbol{\beta})=1-\left\{1-\exp \left(-\exp \left(\frac{y-\mathbf{x} \boldsymbol{\beta}}{\delta}\right)\right)\right\}^{\theta} .
$$

Como casos particulares do modelo dado por (3.5), temos os modelos de regressão exponencial para $\theta=\delta=1$ e para $\theta=1$ temos o modelo de regressão Weibull. 


\subsection{Análise clássica para os modelos de regressão Weibull- exponenciada}

Nesta seção será apresentada uma análise clássica para a determinação de estimadores de máxima verossimilhança e intervalos de confiança para os parâmetros envolvidos no modelo de regressão dado em (3.5).

\subsubsection{Estimação dos parâmetros por máxima verossimilhança}

Sejam $\left(y_{1}, \mathbf{x}_{1}\right), \ldots,\left(y_{n}, \mathbf{x}_{n}\right), n$ observaçoes independentes do modelo dado por (3.3), onde $y_{i}$ representa o logarítmo do tempo de vida ou logarítmo do tempo censura, e $\mathrm{x}_{i}=$ $\left(x_{i 1}, \ldots, x_{i p}\right)$ o vetor de covariáveis associadas ao $i$-ésimo indivíduo. Como antes, $F$ e $C$ denotam o conjunto de indivíduos (ítens) que resultaram em falha ou censura, respectivamente. A funçâo log-verossimilhança para $(\boldsymbol{\beta}, \delta, \theta)$ é dada por

$$
\begin{aligned}
\ell(\boldsymbol{\beta}, \theta, \delta)= & r \log (\theta)-r \log (\delta)+(\theta-1) \sum_{i \in F} \log \left[1-\exp \left\{-\exp \left\{\frac{y_{i}-\mathbf{x}_{\mathbf{i}} \beta}{\delta}\right\}\right\}\right]+ \\
& +\sum_{i \in F}\left(\frac{y_{i}-\mathbf{x}_{\mathbf{i}} \beta}{\delta}\right)-\sum_{i \in F} \exp \left\{\frac{y_{i}-\mathbf{x}_{\mathbf{i}} \beta}{\delta}\right\}+ \\
& +\sum_{i \in C} \log \left[1-\left(1-\exp \left\{-\exp \left(\frac{y_{i}-\mathbf{x}_{\mathbf{i}} \beta}{\delta}\right)\right\}\right)^{\theta}\right],
\end{aligned}
$$

onde $r$ é o numero de observações não censuradas.

Os estimador de máxima verossimilhança de $(\boldsymbol{\beta}, \delta, \theta)$ é obtido máximizando-se a função $\log$-verossimilhança (3.7), o equivale a resolver o seguinte sistema de equações não lineares:

$$
\begin{aligned}
\frac{\partial \ell}{\partial \theta}= & \frac{r}{\theta}+\sum_{i \in F} \log \left[1-e^{-e^{z_{i}}}\right]-\sum_{i \in C} \frac{\left(1-e^{-e^{z_{i}}}\right)^{\theta} \log \left(1-e^{-e^{z_{i}}}\right)}{1-\left(1-e^{-e^{z_{i}}}\right)^{\theta}}=0, \\
\frac{\partial \ell}{\partial \delta}= & -\frac{r}{\delta}-\frac{1}{\delta} \sum_{i \in F} z_{i}+\sum_{i \in F} z_{i} e^{z_{i}}-\frac{(\theta-1)}{\delta} \sum_{i \in F} \frac{z_{i} e^{z_{i}-e^{z_{i}}}}{1-e^{-e^{z_{i}}}}- \\
& \frac{\theta}{\delta} \sum_{i \in C} \frac{z_{i}\left(1-\epsilon^{-e^{z_{i}}}\right)^{\theta-1} \log \left(1-e^{-e^{z_{i}}}\right)}{1-\left(1-e^{-e^{z_{i}}}\right)^{\theta}}=0,
\end{aligned}
$$




$$
\begin{aligned}
\frac{\partial \ell}{\partial \beta_{j}}= & -\frac{1}{\delta} \sum_{i \in F} x_{i j}+\frac{1}{\delta} \sum_{i=1}^{n} x_{i j} e^{z_{i}}-\frac{(\theta-1)}{\delta} \sum_{i \in F} \frac{x_{i j} e^{z_{i}-e^{z_{i}}}}{1-e^{-e^{z_{i}}}}- \\
& \frac{\theta-1}{\delta} \sum_{i \in C} \frac{x_{i j}\left(1-e^{-e^{z_{i}}}\right)^{\theta-1} \log \left(1-e^{-e^{z_{i}}}\right)}{1-\left(1-e^{-e^{z_{i}}}\right)^{\theta}}=0, \quad j=1, \ldots, p,
\end{aligned}
$$

onde $z_{i}=\frac{\left(y_{i}-\mathbf{x}_{\mathbf{i}} \boldsymbol{\beta}\right)}{\delta}$. Como não é possível obter de modo análitico os valores de $\hat{\beta}_{1}, \ldots, \hat{\beta}_{p}$, $\hat{\delta}$ e $\hat{\theta}$ que satisfazem as equações acima, pode-se recorrer a métodos iterativos, como por exemplo o método de Newton-Rapson para calcular estimativas dos parâmetros.

\subsubsection{Inferência sobre os parâmetros $\beta_{1}, \ldots, \beta_{p}, \delta$ e $\theta$}

Para inferência sobre os parâmetros de um determinado modelo, usualmente, utilizase a normalidade assintótica dos estimadores de máxima verossimilhança. Assim para $\hat{\beta}_{1}, \ldots, \hat{\beta}_{p}, \hat{\delta}$ e $\hat{\theta}$ EMVs de $\beta_{1}, \ldots, \beta_{p}, \delta$ e $\theta$ sob certas condições de regularidade (veja, Cox e Hinkley, 1974) tem-se o seguinte resultado para $n \longrightarrow \infty$ :

$$
i^{1 / 2}(\boldsymbol{\beta}, \delta, \theta)\left[\left(\begin{array}{c}
\hat{\beta}_{1} \\
\vdots \\
\hat{\beta}_{p} \\
\hat{\delta} \\
\hat{\theta}
\end{array}\right)-\left(\begin{array}{c}
\beta_{1} \\
\vdots \\
\beta_{p} \\
\delta \\
\theta
\end{array}\right)\right] \stackrel{D}{\longrightarrow} N\left(0, I_{p+2}\right)
$$

onde $i^{1 / 2}(\beta, \delta, \theta)$ é a matriz raiz quadrada da matriz de informação de Fisher e $I_{p+2}$ é a matriz identidade de ordem $(p+2) \times(p+2)$. A normalidade assintótica (3.11) segue sendo válida se $i(\boldsymbol{\beta}, \delta, \theta)$ é substituido por seu estimador consistente $i(\hat{\boldsymbol{\beta}}, \hat{\delta}, \hat{\theta})$ ou simplemente se considerarmos a matriz de informação avaliada nos estimadores de máxima verossimilhança dada por 


$$
I(\hat{\boldsymbol{\beta}}, \hat{\delta}, \hat{\theta})=\left[\begin{array}{ccccc}
-\frac{\partial^{2} \ell}{\partial \beta_{1}} & \ldots & -\frac{\partial^{2} \ell}{\partial \beta_{1} \partial \beta_{p}} & -\frac{\partial^{2} \ell}{\partial \beta_{1} \partial \delta} & -\frac{\partial^{2} \ell}{\partial \beta_{p} \partial \theta} \\
\vdots & \ddots & \vdots & \vdots & \vdots \\
-\frac{\partial^{2} \ell}{\partial \beta_{p} \partial \beta 1} & \cdots & -\frac{\partial^{2} \ell}{\partial \beta_{p}^{2}} & -\frac{\partial^{2} \ell}{\partial \beta_{p} \partial \delta} & -\frac{\partial^{2} \ell}{\partial \beta_{p} \partial \theta} \\
& & & -\frac{\partial^{2} \ell}{\partial \delta^{2}} & -\frac{\partial^{2} \ell}{\partial \delta \partial \theta} \\
\text { simétrica } & & & & -\frac{\partial^{2} \ell}{\partial \theta^{2}}
\end{array}\right]_{\left(\beta_{, \delta, \theta)=(\hat{\beta}, \hat{\delta}, \hat{\theta})}\right.} .
$$

Considerando a distribuição normal assintótica (3.11) para os estimadores de máxima verossimilhança $\hat{\boldsymbol{\beta}}, \hat{\delta}$ e $\hat{\theta}$ considerando um nível de confiança $100(1-\alpha) \%$, intervalos de confiança são dados por:

$$
\begin{aligned}
i c\left(\beta_{j}\right) & =\left(\hat{\beta}_{j}-z_{\alpha / 2}\left(I^{(j j)}\right)^{1 / 2} ; \hat{\beta}_{j}+z_{\alpha / 2}\left(I^{(j j)}\right)^{1 / 2}\right), \quad j=1, \ldots, p \\
i c(\delta) & =\left(\hat{\delta}-z_{\alpha / 2}\left(I^{(p+1 p+1)}\right)^{1 / 2} ; \hat{\delta}+z_{\alpha / 2}\left(I^{(p+1 p+1)}\right)^{1 / 2}\right) \\
i c(\theta) & =\left(\hat{\theta}-z_{\alpha / 2}\left(I^{(p+2 p+2)}\right)^{1 / 2} ; \hat{\theta}+z_{\alpha / 2}\left(I^{(p+2 p+2)}\right)^{1 / 2}\right)
\end{aligned}
$$

onde $I^{(j j)}$ é o $j$-ésimo elemento diagonal da matriz $I^{-1}(\hat{\boldsymbol{\beta}}, \hat{\delta}, \hat{\theta}), j=1, \ldots, p$ e $z_{\alpha / 2}$ é o percentil da distribuição normal padrão correspondente ao coefeciente de confiança estabelecido.

\subsubsection{Teste para modelo de regressão Weibull}

Se consideramos as seguintes hipóteses

$$
H_{0}: \theta=1 \text { versus } H_{1}: \theta \neq 1 \text {, }
$$

estaremos testando a adequação do modelo de regressão Weibull para os tempos de vida. Usamos especificamente a estatística

$$
R V=-2 \log \left\{\frac{L(1, \tilde{\delta}, \tilde{\boldsymbol{\beta}})}{L(\hat{\theta}, \hat{\delta}, \hat{\boldsymbol{\beta}})}\right\},
$$

onde $L(1, \tilde{\delta}, \tilde{\boldsymbol{\beta}})$ corresponde ao máximo de $L(1, \delta, \boldsymbol{\beta})$ dado por

$$
L(1, \delta, \boldsymbol{\beta})=\prod_{i \in F} \frac{1}{\delta} \exp \left\{z_{i}-e^{z_{i}}\right\} \prod_{i \in C} e^{-e^{z_{i}}}
$$


sendo $z_{i}=\left(y_{i}-\mathrm{x} \boldsymbol{\beta}\right) / \delta, i=1, \ldots, n$. A estatística (3.17) para grande amostras, tem distribuição qui-quadrado com um grau de liberdade sob a hipóteses $H_{0}$ (veja Rao, 173).

\subsubsection{Teste para o modelo de regressão exponencial}

Considerar $H_{0}: \theta=1, \delta=1$ versus, $H_{1}: \theta \neq 1$, ou $\delta \neq 1$ equivale a testar se os dados admitem o modelo de regressão exponencial para $T$ e a estatística usada para o teste é

$$
R V=-2 \log \left\{\frac{L(1,1, \tilde{\boldsymbol{\beta}})}{L(\hat{\theta}, \hat{\delta}, \hat{\boldsymbol{\beta}})}\right\},
$$

onde $\tilde{\beta}$ é o valor que maximiza a verossimilhança

$$
\left.L(1,1, \boldsymbol{\beta})=\prod_{i \in F} \exp \left\{\left(y_{i}-\mathbf{x} \boldsymbol{\beta}\right)-\exp \left\{y_{i}-\mathbf{x}_{i} \boldsymbol{\beta}\right)\right\}\right\} \prod_{i \in C} \exp \left\{-\exp \left(y_{i}-\mathbf{x}_{i} \boldsymbol{\beta}\right)\right\}
$$

A estatística da razão de verossimilhança (3.18), sob $H_{0}$ tem distribuição assintótica quiquadrado com 2 graus de liberdade (veja Rao, 1973).

\subsection{Análise Bayesiana para o modelo de regressão Weibull-exponenciada}

O procedimento clássico usual para análise de modelos de regressão Weibull-exponenciada consiste inicialmente, da utilização de alguns método iterativo para obtenção das estimativas dos parâmetros (estimativas de máxima verossimilhança). Como discutido na seção 3.4 , as distribuições dos estimadores obtidos desta forma somente são conhecidas assintoticamente, o que pode resultar em um problema sério, uma vez que, em geral, trabalha-se com amostras não muito grandes. Desta forma, inferências baseadas nessas estatísticas podem não ser confiáveis, conforme mostra o exemplo 1 com dados simulados na seção 3.6. Um procedimento alternativo que pode contornar esse problema é através da utilização de métodos Bayesianos, que permite a incorporação de conhecimento a priori dos parâmetros do modelo através de uma densidade a priori. 
Nesta seção apresentaremos uma análise Bayesiana do modelo de regressão Weibullexponenciada usando métodos de simulação de Monte Carlo via Cadeias de Markov (MCMC), tais como Gibbs sampling (Casela e George, 1992) e Metropolis-Hasting (Chib e Greenberg, 1995).

\subsubsection{Densidade conjunta a posteriori dos parâmetros do mode- lo de regressão Weibull-exponenciada}

Para representar o grau de conhecimento sobre os parâmetros do modelo de regressão Weibull-exponenciada (3.5), consideramos as seguintes densidades à priori para $\theta$. $\delta$ e $\boldsymbol{\beta}$ :

$$
\begin{aligned}
\theta & \sim \Gamma\left(c_{1}, d_{1}\right), \\
\delta & \sim \Gamma\left(c_{2}, d_{2}\right), \\
\beta_{j} & \sim N\left(\mu_{0 j}, \sigma_{0 j}^{2}\right), \quad j=1, \ldots, p,
\end{aligned}
$$

onde $\Gamma(c, d)$ denota a distribuição Gama com média $c / d, N\left(\mu, \sigma^{2}\right)$ denota a distribuição Normal com média $\mu$ e variância $\sigma^{2}$, e $c_{i}, d_{i}, i=1,2$ e $\mu_{0 j}, \sigma_{0 j}, j=1, \ldots, p$ são constantes conhecidas baseadas na informação a priori do especialista e da análise preliminar dos dados.

Assumindo independência entre os parâmetros a densidade a priori conjunta é dada por:

$$
\pi(\theta, \delta, \boldsymbol{\beta}) \propto \theta^{c_{1}-1} \delta^{c_{2}-1} \exp \left\{-d_{1} \theta-d_{2} \delta-\frac{1}{2} \sum_{j=1}^{p}\left(\frac{\beta_{j}-\mu_{0 j}}{\sigma_{0 j}}\right)^{2}\right\},
$$

onde $\theta>0, \delta>0$ e $-\infty<\beta_{j}<\infty$. Priori não informativa é obtida fazendo $c_{i}=d_{i}=0$, $i=1,2, \mu_{0 j}=0$ e $\sigma_{0 j}^{2} \longrightarrow \infty, j=1, \ldots, p$. Isto resulta em

$$
\pi(\theta, \delta, \boldsymbol{\beta}) \propto \frac{1}{\theta \delta}
$$

De (3.7) pode-se mostrar que a função verossimilhança para $\theta, \delta$ e $\boldsymbol{\beta}$ é dada por $L(\theta, \delta, \boldsymbol{\beta})=\exp \{\ell(\theta, \delta, \boldsymbol{\beta})\}$, isto é,

$$
L(\theta, \delta, \boldsymbol{\beta})=\theta^{r} \delta^{-r} \exp \left\{\sum_{i \in F}\left(\frac{y_{i}-\mathbf{x}_{\mathbf{i}} \beta}{\delta}\right)-\sum_{i \in F} \exp \left\{\frac{y_{i}-\mathbf{x}_{\mathbf{i}} \beta}{\delta}\right\}\right\} \times \Psi_{1}(\theta, \delta, \boldsymbol{\beta}),
$$


onde

$$
\begin{aligned}
\Psi_{1}(\theta, \delta, \boldsymbol{\beta})= & \\
& \prod_{i \in F}\left[1-\exp \left\{-\exp \left(\frac{\left.y_{i}-\mathbf{x}_{\mathbf{i}} \beta\right)}{\delta}\right)\right\}\right]^{\theta-1} \prod_{i \in C}\left[1-\left[1-\exp \left\{-\exp \left(\frac{\left.y-\mathbf{x}_{\mathbf{i}} \beta\right)}{\delta}(\beta) .2 \%\right\}\right)\right.\right.
\end{aligned}
$$

Combinado a densidade a priori conjunta (3.20) com a função verossimilhança (3.21) e o teorema de Bayes pode-se mostrar que a densidade a posteriori conjunta de $\theta, \beta$ e $\beta$ é dada por

$$
\begin{aligned}
\pi(\theta, \delta, \boldsymbol{\beta} \mid D) \propto & \theta^{r+c_{1}-1} \delta^{-r+c_{2}-1} \exp \left\{-d_{1} \theta-d_{2} \delta-\frac{1}{2} \sum_{j=1}^{p}\left(\frac{\beta_{j}-\mu_{0 j}}{\sigma_{0 j}}\right)^{2}\right. \\
& \left.+\sum_{i \in F}\left(\frac{y_{i}-\mathbf{x}_{\mathbf{i}} \beta}{\delta}\right)-\sum_{i \in F} \exp \left\{\frac{y_{i}-\mathbf{x}_{\mathbf{i}} \beta}{\delta}\right\}\right\} \times \Psi_{1}(\theta, \delta, \boldsymbol{\beta})
\end{aligned}
$$

onde $\theta>0, \delta>0, \beta_{j} \in \mathcal{R}, j=1, \ldots, p$ e $D$ é o conjunto de dados. Conjecturamos que a densidade a posteriori conjunta para o caso da priori não informativa é própria embora ainda este seja um problema em aberto.

Notamos aqui que a densidade a posteriori conjunta não é uma densidade padrão. portanto só podemos avaliar as densidades a posteriori marginais por meio de métodos de aproximação, tais como o método de Laplace (Tierney, Kass e Kadane, 1986) ou usando métodos de simulação de Monte Carlo em Cadeias de Markov, tais como Gibbs Sampling (Casela e George, 1992) e Metropolis-Hasting (Chib E Greenberg, 1995). Nesta trabalho optamos pelos métodos de simulação por serem de fácil implementação computacional.

Para obtermos uma amostra a posteriori das densidades a posteriori marginais de $\theta>0, \delta>0, \beta_{j} \in \mathcal{R}, j=1, \ldots, p$, exploramos o uso do método do amostrador de Gibbs que baseia-se em sucessivas gerações das distribuições condicionais a posteriori $\pi(\theta \mid \delta, \boldsymbol{\beta}, D), \pi(\delta \mid \theta, \beta, D)$ e $\pi\left(\beta_{j} \mid \theta, \delta, \beta_{(-j)}, D\right) \operatorname{com} \beta_{(-j)}=\left(\beta_{1}, \ldots, \beta_{j-1}, \beta_{j+1}, \ldots, \beta_{p}\right)$.

Pode-se mostrar que as densidades a posteriori condicionais para o algoritmo de Gibbs são dadas por

$$
\pi(\theta \mid \delta, \boldsymbol{\beta}, D) \propto \theta^{r+c_{1}-1} \exp \left\{\theta\left(B(\delta, \boldsymbol{\beta})-d_{1}\right)\right.
$$




$$
\begin{aligned}
& \left.+\sum_{i \in C} \log \left[1-\left[1-\exp \left\{-\exp \left(\frac{\left.y-\mathbf{x}_{\mathbf{i}} \beta\right)}{\delta}\right)\right\}\right]^{\theta}\right]\right\}, \\
\pi(\delta \mid \theta, \boldsymbol{\beta}, D) \propto & \delta^{-r+c_{2}-1} \exp \left\{-d_{2} \delta+\sum_{i \in F}\left(\frac{y_{i}-\mathbf{x}_{\mathbf{i}} \beta}{\delta}\right)-\right. \\
& \left.\sum_{i \in F} \exp \left\{\frac{y_{i}-\mathbf{x}_{\mathbf{i}} \beta}{\delta}\right\}+\log \left(\Psi_{1}(\theta, \delta, \boldsymbol{\beta})\right)\right\}, \\
\pi\left(\beta_{j} \mid \theta, \delta, \beta_{-j}, D\right) \propto & \exp \left\{-\frac{1}{2}\left(\frac{\beta_{j}-\mu_{0 j}}{\sigma_{0 j}}\right)^{2}-\sum_{i \in F}\left(\frac{x_{j i} \beta_{j}}{\delta}\right)-\right. \\
& \left.\sum_{i \in F} \exp \left\{\frac{y_{i}-\mathbf{x}_{i} \beta}{\delta}\right\}+\log \Psi_{1}(\theta, \delta, \beta)\right\},
\end{aligned}
$$

onde $B(\delta, \boldsymbol{\beta})=\prod_{i \in F}\left[1-\exp \left\{-\exp \left(\frac{\left.y_{\mathbf{i}}-\mathbf{x}_{\mathbf{i}} \beta\right)}{\delta}\right)\right\}\right]$.

Observe que as densidades em (3.24) não tem uma forma padrão conhecida, portanto devemos usar o algoritmo de Metropolis-Hasting dentro do ciclo do algoritmo de Gibbs para gerar $\theta, \delta$ e $\beta_{j}, j=1, \ldots, p$.

\subsubsection{Estimador de Bayes da função de sobrevivência no modelo de regressão Weibull-exponenciada}

Considerandos as amostras Gibbs geradas em (3.24) de $\theta, \delta$ e $\beta$, pode-se obter estimativas de Monte Carlo para os momentos de interesse. Um caso especial deste é dado pela função de sobrevivência (3.4) para valores da covariáveis $\boldsymbol{x}_{h}$ dada. Um estimador de Bayes para $S\left(Y_{h}\right)$ com respeito à função perda quadrática é dada por

$$
E\left[S\left(Y_{h} \mid D\right)\right]=\int\left[1-\left\{1-\exp \left(-\exp \left(\frac{Y_{h}-\mathrm{x}_{h} \boldsymbol{\beta}}{\delta}\right)\right)\right\}^{\theta-1}\right] \pi(\theta, \delta, \boldsymbol{\beta} \mid D) d \theta d \delta d \boldsymbol{\beta}
$$

Assim, essa integral pode ser aproximada por sua estimativa de Monte Carlo dada por $\hat{S}\left(Y_{h} \mid D\right)=\frac{2}{R S} \sum_{r=1}^{R} \sum_{s=\frac{S}{2}+1}^{S}\left[1-\left\{1-\exp \left(-\exp \left(\frac{y_{h}-x_{1 h} \beta_{1}^{(r, s)}-\ldots-x_{p h} \beta_{p}^{(r, s)}}{\delta(r, s)}\right)\right)\right\}^{\theta^{(r, s)}-1}\right]$,

onde $\theta^{(r, s)}, \delta^{(r, s)}$, e $\beta_{1}^{(r, s)}, \ldots, \beta_{p}^{(r, s)}$ denotam amostras de $\theta, \delta$ e $\beta_{1}, \ldots, \beta_{p}$, respectivamente na $r$ - ésima iteração e na $s$ - ésima replica, $R$ e $S$ são respectivamente, número total de 
iterações e simulações no amostrador de Gibbs e $\mathrm{x}_{h}$ é usualmente a mediana dos valores da covariável. Similarmemte, pode-se determinar uma estimativa para a função risco.

\subsubsection{Densidade conjunta a posteriori dos parâmetros do mode- lo de regressão Weibull}

Consideramos as seguintes densidades a priori para $\delta$ e $\beta$ :

$$
\begin{aligned}
\delta & \sim \Gamma\left(c_{2}, d_{2}\right) . \\
\beta_{j} & \sim N\left(\mu_{0 j}, \sigma_{0 j}^{2}\right), \quad j=1, \ldots, p,
\end{aligned}
$$

onde $\Gamma(c, d)$ denota a distribuição Gama com média $c / d, N\left(\mu, \sigma^{2}\right)$ denota a distribuição Normal com média $\mu$ e variância $\sigma^{2}$, e $c_{2} . d_{2}$, e $\mu_{0 j}, \sigma_{0 j}, j=1, \ldots, p$ são constantes conhecidas baseadas na informação a priori do especialista e da análise preliminar dos dados.

Assumindo independência entre os parâmetros do modelo de regressão Weibull e considerando a função de verossimilhança $(3.21) \operatorname{com} \theta=1$, pode-se mostrar que a densidade a posteriori conjunta de $\delta$ e $\boldsymbol{\beta}$ é dada por

$$
\begin{aligned}
\pi(\delta, \boldsymbol{\beta} \mid D) \propto & \delta^{-r+c_{2}-1} \exp \left\{-d_{2} \delta-\frac{1}{2} \sum_{j=1}^{p}\left(\frac{\beta_{j}-\mu_{0 j}}{\sigma_{0 j}}\right)^{2}+\sum_{i \in F}\left(\frac{y_{i}-\mathbf{x}_{\mathbf{i}} \beta}{\delta}\right)-\right. \\
& \left.-\sum_{i=1}^{n} \exp \left\{\frac{y_{i}-\mathbf{x}_{\mathbf{i}} \beta}{\delta}\right\}\right\},
\end{aligned}
$$

onde $\delta>0, \beta_{j} \in \mathcal{R}, j=1, \ldots, p$ e $D$ é o conjunto de dados.

Para obtermos uma amostra a posteriori das densidades marginais a posteriori de $\delta>$ $0, \beta_{j} \in \mathcal{R}, j=1, \ldots, p$, exploramos o uso do algoritmo Gibbs que baseia-se em sucesivas gerações das distribuições condicionais a posteriori $\pi(\delta \mid \theta, \beta, D)$ e $\pi\left(\beta_{j} \mid \theta, \delta, \beta_{(-j)}, D\right)$ com $\beta_{(-j)}=\left(\beta_{1}, \ldots, \beta_{j-1}, \beta_{j+1}, \ldots, \beta_{p}\right)$.

Pode-se mostrar que as densidades a posteriori condicionais para o algoritmo de Gibbs 
são dadas por

$$
\begin{aligned}
\pi(\delta \mid, \beta, D) & \propto \delta^{-r+c_{2}-1} \exp \left\{-d_{2} \delta+\sum_{i \in F}\left(\frac{y_{i}-\mathbf{x}_{\mathbf{i}} \beta}{\delta}\right)-\sum_{i=1}^{n} \exp \left\{\frac{y_{i}-\mathbf{x}_{\mathbf{i}} \beta}{\delta}\right\}\right\},(3 \\
\pi\left(\beta_{j} \mid \delta, \beta_{-j}, D\right) & \propto \exp \left\{-\frac{1}{2}\left(\frac{\beta_{j}-\mu_{0 j}}{\sigma_{0 j}}\right)^{2}-\sum_{i \in F}\left(\frac{x_{j i} \beta_{j}}{\delta}\right)-\sum_{i=1}^{n} \exp \left\{\frac{y_{i}-\mathbf{x}_{i} \beta}{\delta}\right\}\right\} .
\end{aligned}
$$

Observe que as densidades em (3.28) não tem uma forma padrão conhecida, portanto devemos usar o algoritmo de Metropolis-Hastings para gerar $\delta$ e $\beta_{j}, j=1, \ldots, p$.

\subsection{Exemplos de aplicação}

O objetivo desta seção é ilustrar a metodologia apresentada nas seções anteriores, através da análise de 2 conjuntos de dados

\subsubsection{Exemplo 1-Dados simulados}

Na tabela 3.1 , temos 30 observações geradas da densidade (3.3) com $z=\left(y-\beta_{0}-\beta_{1} x\right) / \delta$ e parâmetros $\theta=4, \delta=2, \beta_{0}=4$ e $\beta_{1}=2$, não incluindo dados censurados.

Tabela 3.1: Dados gerados paro modelo de regressão Weibull-exponenciada.

\begin{tabular}{lllllllll}
\hline \hline$i$ & $x_{i}$ & $y_{i}$ & $i$ & $x_{i}$ & $y_{i}$ & $i$ & $x_{i}$ & $y_{i}$ \\
\hline 1 & 2.0 & 9.746 & 11 & 4.3 & 14.443 & 21 & 14 & 33.409 \\
2 & 2.2 & 8.686 & 12 & 6.0 & 16.701 & 22 & 15 & 35.819 \\
3 & 3.5 & 10.260 & 13 & 4.3 & 15.614 & 23 & 16 & 37.574 \\
4 & 4.4 & 15.224 & 14 & 6.3 & 16.649 & 24 & 17 & 38.317 \\
5 & 5.4 & 15.224 & 15 & 8.0 & 21.615 & 25 & 21 & 46.948 \\
6 & 6.6 & 17.051 & 16 & 9 & 23.089 & 26 & 25 & 56.662 \\
7 & 7.0 & 19.883 & 17 & 10 & 25.013 & 27 & 24 & 50.382 \\
8 & 8.5 & 21.571 & 18 & 11 & 28.214 & 28 & 16 & 37.283 \\
9 & 9.0 & 21.264 & 19 & 12 & 27.392 & 29 & 15 & 35.422 \\
10 & 7.8 & 19.275 & 20 & 13 & 32.209 & 30 & 25 & 54.480 \\
\hline \hline
\end{tabular}




\section{Análise clássica}

Considerando a log-verossimilhança dada em (3.7) temos que o estimador de máxima verossimilhança do parâmetro $\eta=\left(\theta, \delta, \beta_{0}, \beta_{1}\right)$ é obtido pela resolução do sistema de equações nào linerares dada em (3.8). Isto é feito através da rotina de Newton-Rapson do pacote $\mathrm{Ox}$.

As estimativas de máxima verossimilhança são dadas por

$$
\hat{\theta}=3,566(5,3619), \quad \hat{\delta}=1,781(2,4623), \quad \hat{\beta}_{0}=4,123(2,1447), \text { e } \hat{\beta}_{1}=1,99(0,0295) \text {. }
$$

Cada valor entre parênteses ao lado das estimativas representa a raiz quadrada do correspondente elemento da diagonal de $I^{-1}\left(\hat{\theta}, \hat{\delta}, \hat{\beta}_{0}, \hat{\beta}_{1}\right)$, onde $I\left(\theta, \delta, \beta_{0}, \beta_{1}\right)$ é a matriz de informação observada. Se admitimos a normalidade assintótica para a distribuição dos estimadores de máxima verossimilhança, $I^{-1}\left(\hat{\theta}, \hat{\delta}, \hat{\beta}_{0}, \hat{\beta}_{1}\right)$ corresponde à estimativa da matriz de covariâncias assintótica, e pode ser utilizada para computar inferências sobres os parâmetros.

A estatística de razão de razão de verosimilhanças para testar $H_{0}: \theta=1 \mathrm{em} \mathrm{(3.16)}$ resultou em $R V=1,06844$, e indica a aceitação do modelo Weibull a um nível de significância $0,05\left(\chi_{(1,0,05)}^{2}=3,8441\right)$.

\section{Análise bayesiana}

Para analisarmos os dados da tabela 3.1, desde uma perspectiva Bayesiana consideramos a densidade a priori para $\theta, \delta, \beta_{0}$ e $\beta_{1}$ dada em (3.19) com $c_{1}=4, d_{1}=1, c_{2}=32, d_{2}=16$, $\mu_{00}=4, \sigma_{00}^{2}=3, \mu_{01}=2$ e $\sigma_{01}^{2}=0,009$ (a escolha desses valores para os parâmetros da densidade a priori, foram baseadas numa opinião experiente combinada com uma análise preliminar dos dados). A partir das densidades condicionais (3.24), geramos para cada um dos parâmetros do modelo de regressão Weibull-exponenciada 10 cadeias separadas de Gibbs cada uma com 2.000 iterações, e devido ao fato de todas as densidades condicionais marginais não apresentarem uma forma padrão conhecida utilizamos então o algoritmo 
de Metropolis-Hasting dentro do ciclo do amostrador de Gibbs. Com o objetivo de diminuir o efeito de pontos iniciais deprezam-se as 1.000 pimeiras iterações e a partir de daí escolhem-se as iterações de 10 em 10 , ou seja, para cada parâmetro considera-se a 1.010 - ésima, . ., 2.000 - ésima iterações; daí as 10 cadeias fornecem um amostra Gibbs de tamanho 1.000 para cada um dos parâmetros. A convergência das amostras geradas pelo algoritmo de Gibbs com Metropolis-Hasting foi monitorada utilizando-se o método proposto por Gelman e Rubin (1992) o qual se baseia na técnica de analise de variância.

Na tabela 3.2, apresenta-se um resumo das distribuições marginais a posteriori aproximadas e as estimativas dos fatores de redução de escala potencial $\hat{R}$ (ver por exemplo. Gelman e Rubin, 1992). Observamos na tabela 3.2 , que $\sqrt{\hat{R}}$ sào bem menores que 1.1 , o que nos indica que o algoritmo de Gibbs com Metropolis-Hasting geram para cada parâmetro amostras que convergem em distribuição para as distribuições condicionais marginais em (3.24).

Tabela 3.2: Sumário a posteriori dos parâmetros do modelo de Regressão Weibullexponenciada

\begin{tabular}{|cccccc|}
\hline \hline & Média & Mediana & D.P. & Intervalo de credibilidade $95 \%$ & $\hat{R}$ \\
\hline$\beta_{0}$ & 3,8506 & 3,8403 & 0,4469 & $(2,9932 ; 4,7162)$ & 1,00667 \\
$\beta_{1}$ & 1,9993 & 1,9998 & 0,0288 & $(1,9382 ; 2,0563)$ & 1,00008 \\
$\delta$ & 2,0087 & 1,9862 & 0,2913 & $(1,4209 ; 2,6573)$ & 1,00156 \\
$\theta$ & 3,9814 & 3,9459 & 0,7481 & $(1,0779 ; 5,6109)$ & 1,00089 \\
\hline \hline
\end{tabular}

Na figura 3.2 , representa-se as densidades a posteriori marginais aproximadas considerando os 1.000 pontos amostrais. Note que os desvios padrões obtidos com o enfoque Bayesiano são bem menores do que os obtidos com o método de máxima verossimilhaça.

Com a finalidade de selecionar entre o modelo de regressão Weibull e o modelo de regressão Weibull-exponenciada o que se ajusta melhor aos dados da tabela (3.1) desde uma perspectiva Bayesiana (o teste de hipóteses dada em (3.16)), utilizamos a técnica 

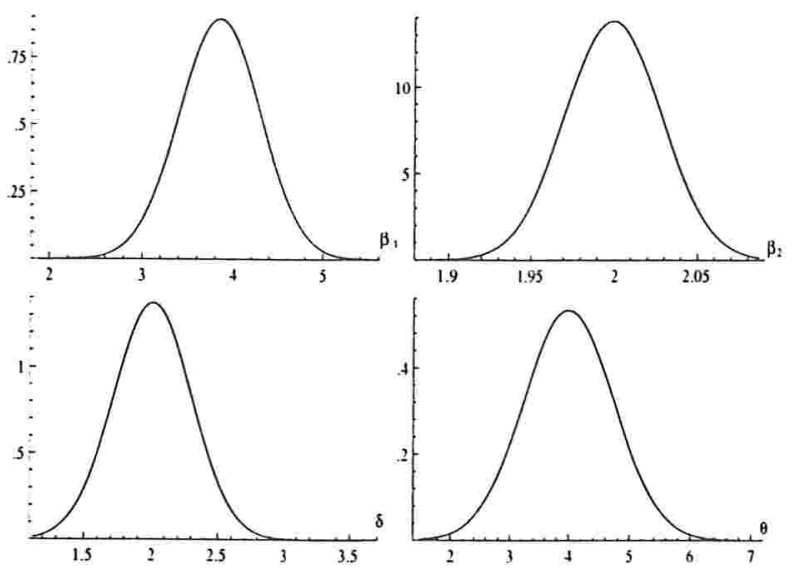

Figura 3.2: Densidades marginais a posteriori para os parâmetros do modelo de regressão Weibull-exponenciado, $\beta_{0}, \beta_{1}, \delta$ and $\theta$

das densidades preditivas condicionadas ordenadas (CPO) (veja capítulo 1). Para isso, consideramos a partir das estimativas do CPO a estimativa da verossimilhança preditiva para definir o pseudo-fator de Bayes. A estimativa da verossimilhança preditiva do modelo de regressào Weibull-exponenciada resultou em $\hat{f}\left(\underline{y} \mid M_{1}\right)=1.792 \times 10^{-20}$, e para o modelo de regressão Weibull essa verossimilhança resultou em $\hat{f}\left(\underline{y} \mid M_{0}\right)=1.47 \times 10^{-28}$. O pseudofator de Bayes do modelo de regressão Weibull com respeito o modelo de regressão Weibullexponenciada resultou em $P S F B_{01}=2.8031 \times 10^{-9}<1$ o qual indica que o modelo de regressão Weibull não é adequado para ajustar os dados da tabela 3.1 e esse resultado contraria o resultado do teste de hipoteses clássica (veja análise clássica acima). Este resultado nos mostra que parece ser temerário utilizar a distribuição assintótica do teste da razão de verossimilhança, pois ela está rejeitando uma hipótese verdadeira; resultados de simulação como o teste da razão parecem indicar que este apresenta níveis de significância bem maiores que os níveis teóricos que são estabelecidos.

O procedimento gráfico descrito no capítulo 1, é adotado para verificarmos o melhor modelo entre os modelos estudados. Considerando os dados da tabela 3.1 , apresenta-se na tabela 3.3 , os valores das estimativas da densidade preditivas condicionadas ordenadas 
$c_{i}(l)=\hat{f}\left(y_{i} \mid D_{(i)}\right), i=1, \ldots, n$ aproximados por seu estimador de Monte Carlo baseadas nas amostras Gibbs, para cada modelo.

Na figura 3.3, apresenta-se os gráficos dos $c_{i}(l)$ contra os $i$ para os modelos de regressão Weibull e Weibull-exponenciada. Da tabela 3.3 e figura 3.3 , observamos que o melhor modelo é o modelo Weibull-exponenciada.

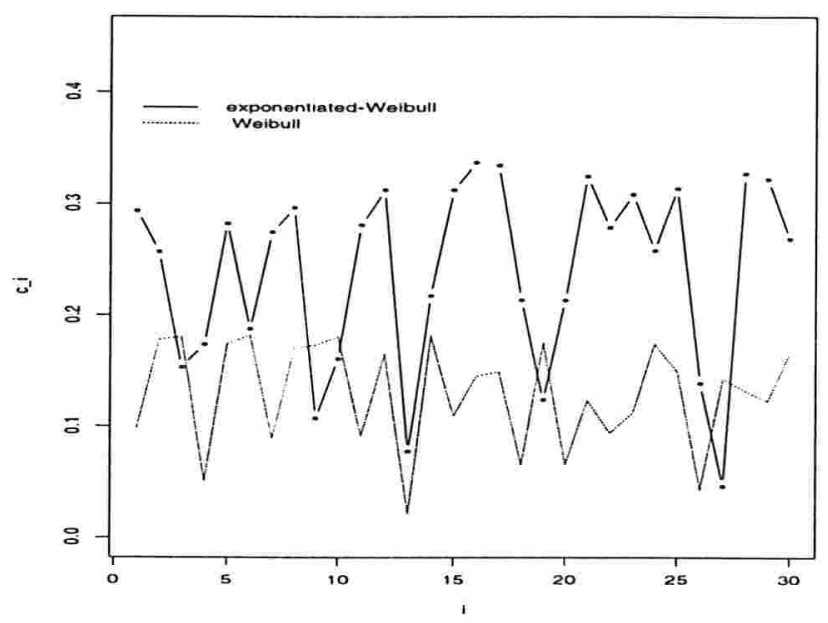

Figura 3.3: Plot de $c_{i}(l)$ versus $i$

\subsubsection{Exemplo 2-Teste de vida de Isolantes}

Os dados da tabela 3.4 , são resultados de um teste acelerado de vida para isolantes de sistemas de uma nova classe $\mathbf{H}$, e referentes ao tempo em horas que os isolantes levaram para tornar-se defeituosos. Os testes foram utilizadas em pequenos motores, para temperaturas elevadas. Dez motores foram colocadas para trabalhar a temperaturas de $190,220,240$, e $260{ }^{\circ} \mathrm{C}$, e inspecionados periodicamente para detectar a ocorrência de falha. Os Dados da tabela 3.4 são referentes à média entre o tempo em que a falha foi observada e o tempo de inspeção anterior. Os tempos entre checagem foram 7, 4, e 2 dias para as respectivas temperaturas. Estes dados foram analisados por Nelson (1990), que considerou o modelo de regressão log-normal para o ajuste dos tempos de vida. 


\begin{tabular}{|l|cc|}
\hline$i$ & Weibull $\left(c_{1}(1)\right)$ & Weibull-exponenciada $\left(c_{i}(2)\right)$ \\
\hline 1 & 0.0986040816 & 0.2935935436 \\
2 & 0.1784192508 & 0.2569415188 \\
3 & 0.1806369442 & 0.1532483601 \\
4 & 0.0507562589 & 0.1736054647 \\
5 & 0.1743275337 & 0.2823201432 \\
6 & 0.1820799199 & 0.187552296 \\
7 & 0.0889940721 & 0.2744424237 \\
8 & 0.1705153204 & 0.2966126858 \\
9 & 0.1735079874 & 0.1074380815 \\
10 & 0.1808018307 & 0.1605345992 \\
11 & 0.0918807093 & 0.2808851279 \\
12 & 0.1657797775 & 0.312313336 \\
13 & 0.0214895793 & 0.0774456402 \\
14 & 0.1816987583 & 0.2175871757 \\
15 & 0.1095415567 & 0.3124269618 \\
16 & 0.1454376905 & 0.3370757668 \\
17 & 0.1494318965 & 0.334371381 \\
18 & 0.0652862838 & 0.2136894111 \\
19 & 0.1757263783 & 0.1238903749 \\
20 & 0.0656442795 & 0.2133202441 \\
21 & 0.1238354895 & 0.3244610886 \\
22 & 0.0940349458 & 0.2788427441 \\
23 & 0.1123034737 & 0.3083304641 \\
24 & 0.1745519103 & 0.2580601204 \\
25 & 0.149597314 & 0.3134202149 \\
26 & 0.0431443935 & 0.1390255184 \\
27 & 0.1429360415 & 0.0460396558 \\
28 & 0.1322505672 & 0.3270160164 \\
29 & 0.1228709264 & 0.3220973127 \\
30 & 0.1655935696 & 0.2685402203 \\
\hline$c(\ell)$ & $1.47 \times 10^{-28}$ & $1.792 \times 10^{-20}$ \\
\hline \hline & & \\
\hline
\end{tabular}

Tabela 3.3: Valores das estimativas $\operatorname{los} c_{i}(l), i=1 \ldots, n$ para os modelos de regressão Weibull e Weibull-exponenciada 
Tabela 3.4: Tempo de vida de isolantes (horas) para cada temperatura, em graus centígrados, com + indicando censura.

\begin{tabular}{cccc}
\hline \hline \multicolumn{4}{c}{ Temperatura ${ }^{0} \mathrm{C}$} \\
\hline 190 & 220 & 240 & 260 \\
\hline \hline 7228 & 1764 & 1175 & 1128 \\
7228 & 2436 & 1521 & 1464 \\
7228 & 2436 & 1569 & 1512 \\
8448 & $2436^{+}$ & 1617 & 1632 \\
9167 & 2436 & 1665 & $1632^{+}$ \\
9167 & 2436 & 1665 & $1632^{+}$ \\
9167 & 3108 & 1713 & $1632^{+}$ \\
9167 & 3108 & 1761 & $1632^{+}$ \\
10511 & 3108 & $1881^{+}$ & $1632^{+}$ \\
10511 & 3108 & 1953 & 1896 \\
\hline
\end{tabular}

\section{Análise clássica}

Transformando a temperatura para assumir o relacionamento Arrhenius, conforme sugerido na literatura de engenharia, usamos o modelo

$$
y_{i}=\beta_{0}+\beta_{1} x_{i}+\delta z_{i}, \quad i=1, \ldots, 40
$$

onde $y_{i}=\log ($ tempo de vida do isolante $i), x_{i}=1000 /\left(\right.$ temperatura $^{0} C$ do $i$-ésimo motor+ $273.20), \beta_{0}, \beta_{1}$ e $\delta$ são parâmetros desconhecidos e $Z_{i}$ tem distribuição dada em (3.3) com parâmetro $\theta$. Para estimar os parâmetros, consideramos o mesmo procedimento dado anteriormente.

Como resultado as estimativas de máxima verossimilhança são dados por $\hat{\beta}_{0}=-4,7261(1,5351), \hat{\beta}_{1}=6,0491(0,4293), \hat{\delta}=0,6264(0,52895), \hat{\theta}=8,2099(19,21)$ Para estudar a possibilidade de utilizar o modelo de regressão Weibull para os dados da tabela 3.4. Consideramos o teste de hipótese $\left(H_{0}: \theta=1\right.$ vs $\left.H_{1}: \theta \neq 1\right)$ e temos que a estatística da razão de verossimilhanças (3.17), resultou em, $R V=1,38319$ e este resultado implica que a o modelo Weibull é estatísticamente adequado para ajustar os dados ao nível de significância de $5 \%$. 
Análise Bayesiana para os tempos de vida de isolantes consideramos o modelo de regressão Weibull-exponenciada

Para analisarmos os dados da tabela 3.4, desde uma perpectiva Bayesiana consideramos o modelo de regressão Weibull-exponenciada com as densidades a priori para $\theta, \delta, \beta_{0}$ e $\beta_{1}$ dadas em (3.19) com $c_{1}=0,186, d_{1}=0,0227, c_{2}=1,422, d_{2}=2,293, \mu_{00}=$ $-3,8415, \sigma_{00}^{2}=1,5, \mu_{01}=5.95$ e $\sigma_{01}^{2}=10,5$. A partir da densidades condicionais (3.24) geramos uma amostra Gibbs através do algoritmo Gibbs com Metropolis-Hasting da seguinte forma: Geram-se 20 cadeias paralelas cada um com 2.000 iterações. Desprezamse as 1.000 primeiras iterações. com objetivo de diminuir o efeito dos pontos iniciais e a partir de daí escolhem-se as iterações de $20 \mathrm{em} 20$, ou seja, para cada parâmetro em cada cadeia considera-se as iterações 1.020 - ésima, ..,2.000 - ésima que resulta em uma amostra de Gibbs de tamanho 2.000. Para monitorar a convergência da cadeia utiliza-se a técnica de Gelman e Rubin (1992).

Tabela 3.5: Sumário a posteriori dos parâmetros do modelo de Regressão Weibullexponenciada com os dados de tempos de vida de isolantes.

\begin{tabular}{|clllcc|}
\hline \hline & Média & Mediana & D.P. & Intervalo de credibilidade $95 \%$ & $\hat{R}$ \\
\hline$\beta_{0}$ & $-4,6923$ & $-4,6763$ & 0,1815 & $(-5,0688 ;-4,3644)$ & 1,0161108 \\
$\beta_{1}$ & 6,0014 & 6,0017 & 0,0602 & $(5,8846 ; 6,1177)$ & 1,000646 \\
$\delta$ & 0,69322 & 0,67411 & 0,16662 & $(0,4229 ; 1,04387)$ & 1,013394 \\
$\theta$ & 8,2075 & 8,2023 & 0,2254 & $(7,7734 ; 8,6588)$ & 1,0002416 \\
\hline \hline
\end{tabular}

Na tabela 3.5, apresenta-se o resumo da distribuição a posteriori do modelo de regressão Weibull-exponenciada e na figura 3.4, apresenta-se a densidade aproximada dos parâmetros do modelo de regressão Weibull-exponenciada. Também na tabela 3.5 , tem-se o resultado das estimativas dos fatores de redução de escala potêncial $\hat{R}$ (veja, Gelman e Rubin, 1992) para todos os parâmetros. Os números de iterações considerados foram suficientes para se chegar à convergência pois $\sqrt{\hat{R}}<1.1$ para todos os parâmetros. 

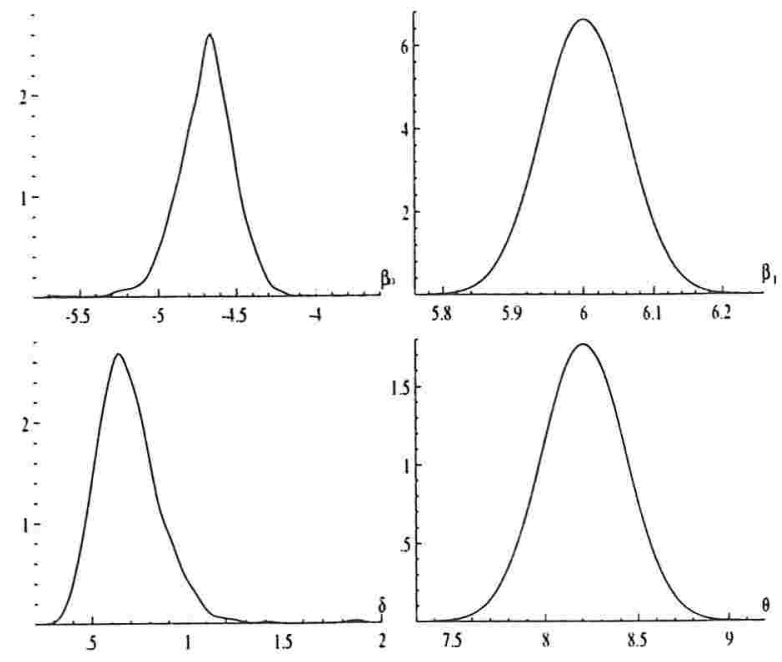

Figura 3.4: Densidades marginais a posteriori aproximados dos parâmetros do modelo de regressão Weibull-exponenciada, $\beta_{0}, \beta_{1}, \delta$ and $\theta$ com os dados de tempo de vida de isolantes

Análise Bayesiana para dos dados de tempos de vida de isolantes considerando o modelo de regressão Weibull

Para analisar os dados da tabela 3.4 agora, consideramos o modelo de regressão Weibull com as densidades a priori (3.26) para os parâmetros, com, $c_{2}=1,653, d_{2}=7,1875$, $\mu_{00}=-3,8415, \sigma_{00}^{2}=3, \mu_{01}=5.9, \sigma_{01}^{2}=10$.

A partir das densidades condicionais marginais (3.28), geramos 20 cadeias separadas de Gibbs cada uma com 2.000 iterações e utilizamos o método proposto por Gelman Rubin (1992) para verificar a convergência das amostras. Para cada cadeia de Markov associada a distribuição de cada um dos parâmetro tomamos uma amostra com a metade das iterações, considerado as iterações 1.020 - ésima, . .,2.000 - ésima, totalizando assim amostras Gibbs de 2.000 pontos amostrais.

As quantidades a posteriori de interesse obtidas a partir das amostras selecionadas são apresentadas na tabela 3.6, onde observamos que as estimativas dos fatores de redução 
potencial são menores de $1.1(\sqrt{\hat{R}}<1.1)$, indicando a convergência das cadeias associadas as amostras geradas.

Tabela 3.6: Sumário a posteriori dos parâmetros do modelo de regressão Weibull

\begin{tabular}{|cccccc|}
\hline \hline & Média & Mediana & D.P. & Intervalo de credibilidade $95 \%$ & $\hat{R}$ \\
\hline$\beta_{0}$ & $-3,8504$ & $-3,8501$ & 0,0480 & $(-3,94370 ;-3,75628)$ & 1,000324 \\
$\beta_{1}$ & 5,9612 & 5,9622 & 0,0263 & $(5,90635 ; 6,00985)$ & 1,003526 \\
$\delta$ & 0,23446 & 0,23336 & 0,00042 & $(0,186117 ; 0,287874)$ & 1,00232 \\
\hline
\end{tabular}

Na figura 3.5 , temos as densidades a posteriori marginais aproximadas para o modelo de regressão Weibull considerando os 2000 pontos amostrais.

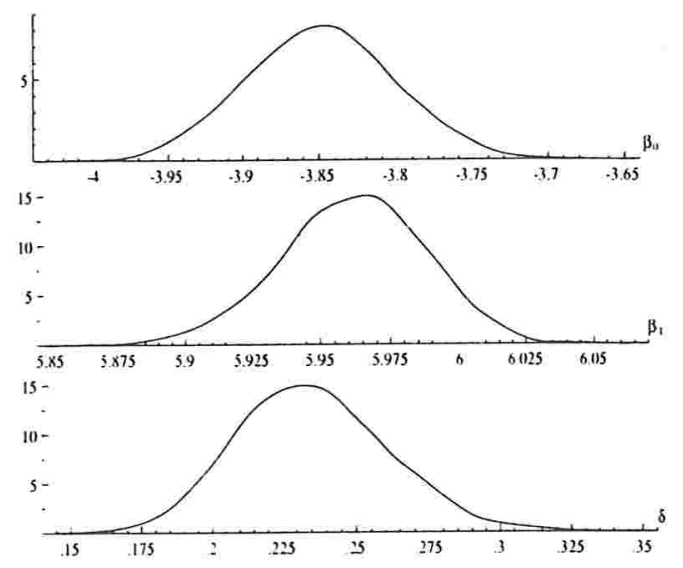

Figura 3.5: Densidades a posteriori aproximados para o modelo de regressão Weibull $\delta$, $\beta_{0}$ e $\beta_{1}$

Usamos o procedimento Bayesiano para verificação da adequabilidade dos modelos de regressão Weibull e Weibull-exponenciada (teste de hipóteses $H_{0}: \theta=1(3.16)$ ) para os dados da tabela 3.4, através das densidades preditivas $C P O_{i}, i=1, \ldots, 40$. Determinouse a estimativas de Monte Carlo da verossimilhança preditiva com as amostras de Gibbs para os modelos de regressão Weibull e Wêtebull-exponenciada e essas quantidades são respectivamente $\hat{f}\left(\underline{y} \mid M_{0}\right)=3.617 \times 10^{-6}$ e $\hat{f}\left(\underline{y} \mid M_{1}\right)=2.403 \times 10^{-17}$. O pseudo-fator 
de Bayes do modelo de regressão Weibull com respeito ao modelo de regressão Weibullexponenciada resultou em, PSF $B_{01}=1.2741 \times 10^{11}>1$, o que indica que o modelo Weibull é bastante adequado para o ajuste dos dados da tabela 3.4, confirmando-se assim o resultado da inferência clássica. Cabe ressaltar que o suporte do enfoque Bayesiano ao modelo de regressão Weibull é bem maior que o apresentado pelo enfoque clássico.

Como na subseção anterior, é também adotado o método gráfico para verificarmos o melhor modelo entre os modelos estudados. Considerando os dados da tabela 3.4. apresenta-se na figura 3.6, o plot do $\log$ da razão dos CPO's dos modelos de regressão Weibull e Weibull-exponenciada com respeito ao numero de observações. Valores positivos do log da razão dos CPO indica a preferência do primero modelo. Observa-se, na figura 3.6 que 36 das 40 observações sustenta ao modelo de regressão Weibull sobre o modelo de regressão Weibull-exponenciada. Este método confirma o resultado obtida acima.

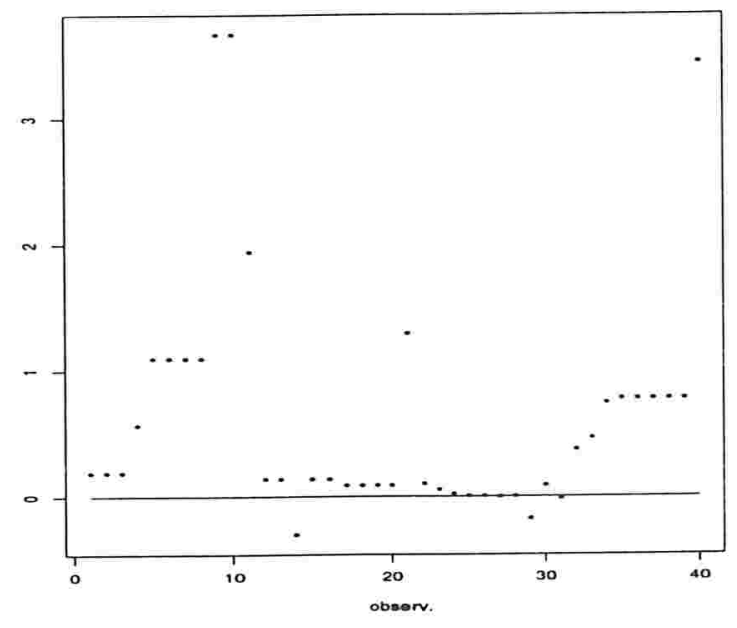

Figura 3.6: Gráfico do log da razão dos CPO's dos modelos de regressão Weibull e Weibullexponenciada versus as observações

Novamente nota-se que o enfoque Bayesiano apresenta uma indicação muito mais forte do que o enfoque clássico de que o modelo Weibull é o mais adequado.

Na figura 3.7, temos a densidade preditiva para o log do tempo de vida futuro, $y$, dada as observações $D$ para um valor da covariável $x_{h}=190^{\circ} C$ aproximada pelo 
método de Monte Carlo com as 2.000 amostras Gibbs dos parâmetros do modelo Weibullexponenciada.

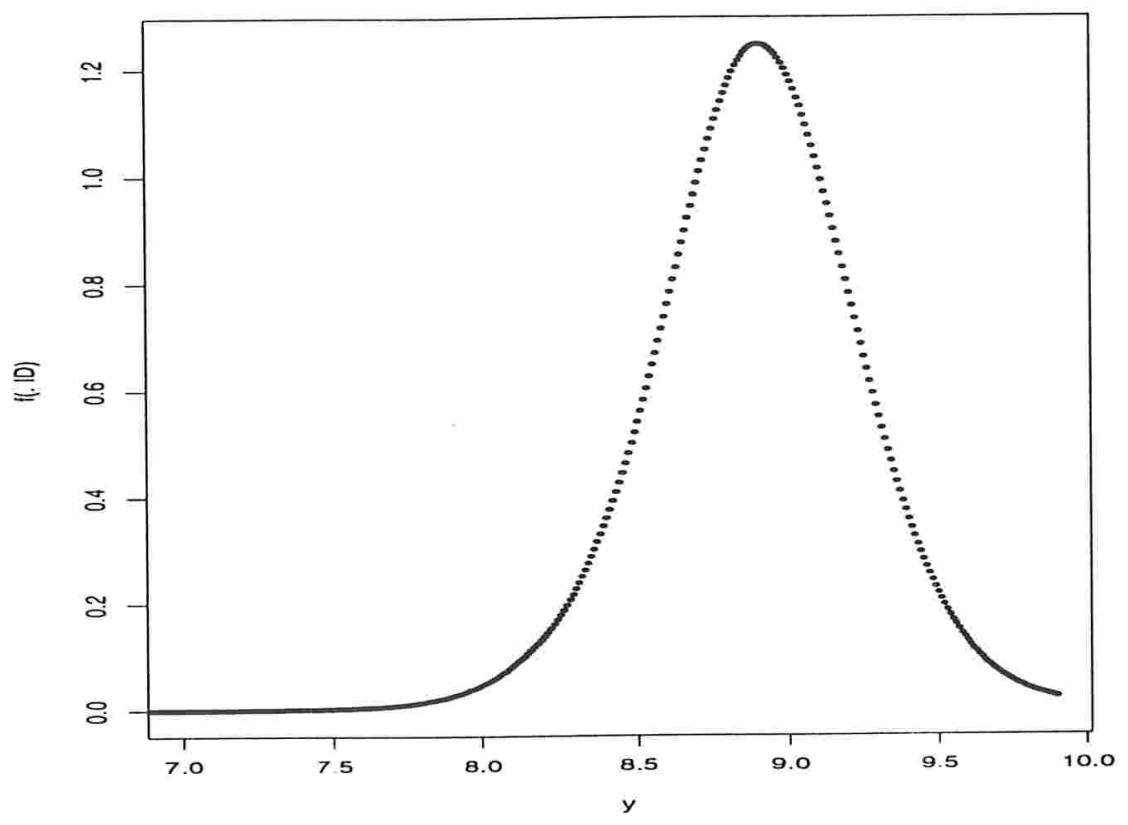

Figura 3.7: Densidade preditiva para o log do tempo de vida futuro no modelo de regressão Weibull-exponenciada

$\mathrm{Na}$ figura 3.8, representamos as funções de sobrevivência dada em (3.6) e de risco dada um valor da covariável $x_{h}=190^{\circ} \mathrm{C}$, aproximadas pelo método de Monte Carlo com as 2.000 amostras Gibbs. 

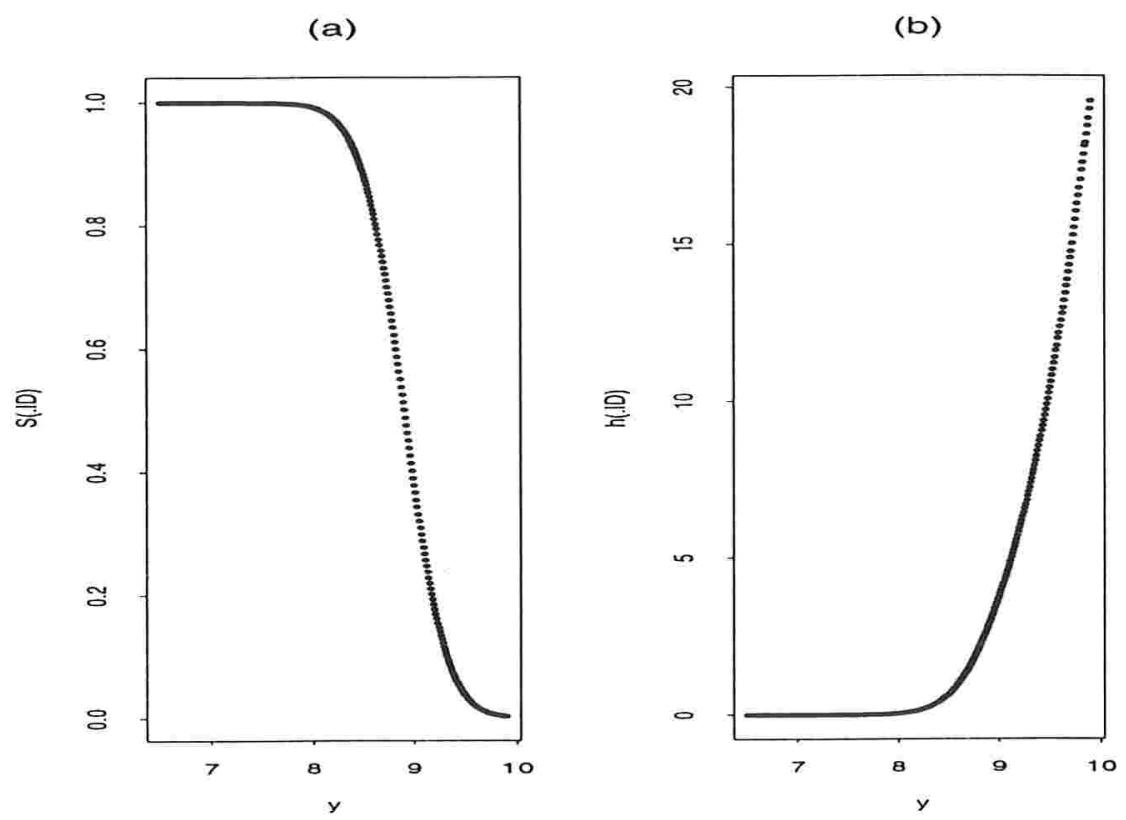

Figura 3.S: Funções de: (a) sobrevivência e (b) risco para o modelo de regressão Weibullexponenciada dado um valor da covariável, $x_{h}=190^{\circ} \mathrm{C}$ 


\section{Capítulo 4}

\section{Uso da distribuição}

\section{Weibull-exponencida para dados com taxas de falha do tipo "bathtub"}

\subsection{Introdução}

Na análise de dados de tempos de vida especialmente no ciclo de vida de um produto pode ocorrer um primeiro período com taxas de falha inicial alta que decresce rapidamente com o tempo (mortalidade infantil) seguida, por um período onde as taxas de falha é aproximadamente constante e uma fase de envelhecimento onde as taxas de falha é crescente devido ao processo de desgate natural do produto, indicado uma função de taxas de falha do tipo "bathtub". Algumas distribuições foram introduzidas para modelar esse tipo de dados, como a família de distribuições gama geralizadas proposta por Stacy(1962), a família de distribuições $F$ geralizadas de Prentice (1975), a distribuição IDB proposta por Horth (1980), que descreve função de taxas de falha crescente (I), descrecente (D), constante e forma de "bathtub" (B), a o qual motivo nome da família. e a família exponencialpotência formulada por Smith e Bain (1975). Uma razoavél e comprensiva revisão para 
modelos com função taxa de falha do tipo "bathtub" foram dadas por Rajarshi e Rajarshi (1988). O procedimento de inferência clássica padrão pode ter fortes dificuldades para esses modelos, especialmente na presença de dados censurados (veja por exemplo, Rajarshi e Rajarshi (1980); Lawless (1982) e Hjorth(1980)).

Conforme visto nos Capítulos anteriores Mudholkar et al.(1995) apresenta uma geralização simples da familia de distribuições Weibull chamada família de distribuição Weibull-exponenciada que além de incluir distribuições com funções de taxas de falha do tipo "bathtub" e unimodal também admite uma ampla classe de distribuições com taxas de falha monótonas. Neste Capitulo comparamos diferentes modelos para análise de dados de tempos de vida com taxas de falha do tipo "bathtub". A distribuição Weibullexponenciada é comparada com a família de distribuições IDB, exponencial-potência e a mistura de distribuições gama. A abordagem usada para o ajuste dos modelos é a abordagem Bayesiana baseada no método de simulação de Monte Carlo via cadeias de Markov (MCMC). A seleção do modelo é implementada usando o pseudo-fator de Bayes. A implementação de um estudo de simulação e a análise de um conjunto de dados reais parece indicar que o modelo Weibull-exponenciada é um dos que apresentam um melhor ajuste para esse tipo de dados.

\subsection{Modelos}

Nesta seção apresentamos algumas das famílias de distribuições mais comuns usadas para modelar tempos de vida com função de taxa de falha de tipo "bathtub". Não discutimos a abordagem de inferência clássica 


\subsubsection{A família de distribuições Weibull-exponenciada}

Conforme visto na seção 2.2 , a função de densidade de probabilidades para a distribuição Weibull-exponenciada é dada por

$$
f(t)=\frac{\alpha \theta}{\sigma}\left[1-\exp \left(-(t / \sigma)^{\alpha}\right)\right]^{\theta-1} \times \exp \left(-(t / \sigma)^{\alpha}\right)(t / \sigma)^{\alpha-1}, \quad 0<t<\infty,
$$

onde $\alpha>0, \theta>0$ são os parâmetros de forma e $\sigma>0$ é o parâmetro de escala. A função de sobrevivência é dada por

$$
S(t)=1-\left[1-\exp \left(-(t / \sigma)^{\alpha}\right)\right]^{\theta}
$$

e a função risco de $T$ é dada por

$$
h(t)=\frac{\alpha \theta[1-\exp (-(t / \sigma))]^{\theta-1} \exp \left(-(t / \sigma)^{\alpha}\right)(t / \sigma)^{\alpha-1}}{\sigma\left[1-\left(1-\exp \left(-(t / \sigma)^{\alpha}\right)\right)^{\theta}\right]} .
$$

A grande flexibilidade deste modelo para ajustar dados de sobrevivência é dado pelas diferentes formas da função risco (4.3) que pode tomar, isto é,

(i) monótona crescente se $\alpha \geq 1$ e $\alpha \theta \geq 1$,

(ii) monótona decrescente se $\alpha \leq 1$ e $\alpha \theta \leq 1$,

(iii) forma de banheira se $\alpha>1$ e $\alpha \theta<1$ e

(iv) unimodal se $\alpha<1$ e $\alpha \sigma>1$,

\subsubsection{A família de distribuições IDB}

A função de sobrevivência da distribuição IBD é dada por

$$
S(t)=\frac{\exp \left\{\delta t^{2} / 2\right\}}{(1+\beta t)^{\theta / \beta+1}}
$$

onde $\delta>0, \theta>0$ e $\beta>0$. 
A função de densidade de probabilidades é

$$
f(t)=\frac{(1+\beta t) \delta t-\theta}{(1+\beta t)^{\theta / \beta+1}} e^{\delta t^{2} / 2}, \quad 0<t<\infty .
$$

A função de taxas de falha é dada por

$$
h(t)=\delta t+\frac{\theta}{1+\beta t} .
$$

Casos especiais da distribuição IDB são dados por:

(a) $\operatorname{Se} \theta=0$, temos a distribuição de Rayleigh;

(b) Se $\delta=\beta=0$, temos a distribuição exponencial;

(c) Se $\delta=0$, temos distribuições com taxas de falha decrescente;

(d) Se $\delta \geq \theta \beta$ distribuições com taxas de falha crescente;

(e) Se $0<\delta<\theta \beta$ distribuições com taxas de falha do tipo "bathtub".

\subsubsection{A família de distribuições exponecial-potência}

Smith e Bain $(1975,1976)$ apresentaram a família de distribuição exponencial-potência com dois parâmetros como uma distribuição alternativa para dados de tempos de vida com taxas de falha do tipo "bathtub". A função de densidade de probabilidade é

$$
f(t)=\beta \alpha^{-\beta} t^{\beta-1} \exp \left(1-e^{\left(\frac{t}{\alpha}\right)^{\beta}}+\left(\frac{t}{\alpha}\right)^{\beta}\right), \quad 0<t<\infty,
$$

onde $\alpha>0$ e $\beta>0$;

A função de distribuição é dada por

$$
F(t)=1-\exp \left(1-e^{\left(\frac{t}{\alpha}\right)^{\beta}}\right)
$$

e a função risco é dada por

$$
h(t)=\frac{f(t)}{1-F(t)}=\alpha^{-\beta} \beta t^{\beta-1} \exp \left(\left(\frac{t}{\alpha}\right)^{\beta}\right)
$$

A função risco (4.9) tem forma de banheira se $0<\beta<1$. 


\subsubsection{Misturas de distribuições Gama}

Glasser (1980) apresentaram a mistura da distribuições gama com parametro de escala comum para os de tempos de vida com função de risco do tipo "bathtub". Neste modelo a função de densidade é dada por

$$
f\left(t ; \alpha_{1}, \alpha_{2}, \beta, p\right)=p f_{1}\left(t ; \alpha_{1}, \beta\right)+(1-p) f_{2}\left(t ; \alpha_{2}, \beta\right)
$$

onde $0<p<1$

$$
f_{j}\left(t ; \alpha_{j}, \beta\right)=\frac{\beta^{\alpha_{j}}}{\Gamma\left(\alpha_{j}\right)} t^{\alpha_{j}-1} e^{\beta t}, \quad \alpha_{j}>0, \quad \beta>0, j=1,2
$$

A função de sobrevivência é dada por

$$
S\left(t ; \alpha_{1}, \alpha_{2}, \beta, p\right)=p S_{1}\left(t ; \alpha_{1}, \beta\right)+(1-p) S_{2}\left(t ; \alpha_{2}, \beta\right), \quad t>0 .
$$

onde $S_{j}\left(t ; \alpha_{j}, \beta\right)$ é a função de sobrvivencia do $j$-ésimo componente.

A função de taxas de falha é de tipo "bathtub" se:

(a) $\alpha_{1}>1, \alpha_{2}=1 ; f_{1}$ estritamente crescente e $f_{2}$ uma exponencial

(b) $\alpha_{1}>1, \alpha_{2}<1$.

\subsection{Inferência Bayesiana}

Assumimos que os tempos de vida são distribuidos independentemente e independentes do mecanismo de censura. Considerando que os dados de tempo de vida são censurados à direita, e que observamos $T_{i}=\min \left(T_{i}^{0}, C_{i}\right)$, onde $T_{i}^{0}$ é o tempo de vida do $i$-ésimo indivíduo e $C_{i}$ é o tempo de censura do $i$-ésima indivíduo, $i=1, \ldots, n$. Neste caso a função de verossimilhança de $\theta$, um vetor de parâmetros de dimensão $p$ é dado por

$$
L(\theta)=\prod_{i \in F} f\left(t_{i} ; \theta\right) \prod_{i \in C} S\left(t_{i} ; \theta\right)
$$


onde $F$ denota o conjunta de observações não censuradas, $C$ denota o conjunto de observações censuradas, $f($.$) e S($.$) é a função de densidade de probabilidades e a função de$ sobreviência, respectivamente.

Considerando uma densidade a prior $\pi(\theta)$ a densidade a posteriori de $\theta$ é dada por

$$
\pi(\theta \mid D)=\frac{L(\theta) \pi(\theta)}{\int L(\theta) \pi(\theta) d \theta}
$$

onde $D$ denota o conjunto de dados observados.

Conforme visto nos capítulos anteriores, na abordagem Bayesiana, inferências são tipicamente baseadas nas densidades a posteriori marginais dos parâmetros envolvidos em (4.12), para os modelos descritos na seçào anterior essas densidades marginais parece que não podem ser obtidas analiticamente. Portanto, para esses casos, métodos de aproximação ou métodos de simulação de Monte Carlo via Cadeia de Markov (MCMC) são necessários. Neste Capítulo optamos pelo segundo método.

\subsubsection{O Modelo Weibull-exponenciada}

Considerando que os dados observados são não censurados, a função de verossimilhança (4.11) é dada por,

$$
L(\alpha, \theta, \sigma) \propto \alpha^{n} \theta^{n} \sigma^{-n \alpha} \exp \left\{-\sum_{i=1}^{n}\left(\frac{t_{i}}{\sigma}\right)^{\alpha}\right\} \prod_{i=1}^{n} t_{i}^{\alpha-1}\left(1-\exp \left\{-\left(\frac{t_{i}}{\sigma}\right)^{\alpha}\right\}\right)^{\theta-1} .
$$

Para análise Bayesiana, assumimos a seguinte densidade a priori para $\alpha, \theta$ e $\sigma$ :

$$
\begin{aligned}
\alpha & \sim \Gamma\left(a_{1}, b_{1}\right), \text { com } a_{1} \text { e } b_{1} \text { conhecidos; } \\
\theta & \sim \Gamma\left(a_{2}, b_{2}\right), \text { com } a_{2} \text { e } b_{2} \text { conhecidos; } \\
\sigma & \sim \Gamma\left(a_{3}, b_{3}\right), \text { com } a_{3} \text { e } b_{3} \text { conheicdos. }
\end{aligned}
$$

onde $\Gamma\left(a_{i}, b_{i}\right)$ denota a distribuição gama com média $\frac{a_{i}}{b_{i}}$ e variância $\frac{a_{i}}{b_{i}^{2}}$. Além disso assumimos independência entre os parâmetros.

Combinando (4.13) e (4.14) e o teorema de Bayes pode-se mostrar que a densidade conjunta a posteriori de $\alpha, \theta$ e $\sigma$ é dada por, 


$$
\begin{aligned}
\pi(\alpha, \theta, \sigma \mid D) \propto & \alpha^{n+a_{1}-1} \theta^{n+a_{2}-1} \sigma^{-n \alpha+a_{3}-1} \exp \left\{-\sum_{i=1}^{n}\left(\frac{t_{i}}{\sigma}\right)^{\alpha}-b_{1} \alpha-b_{2} \theta-b_{3} \sigma\right\} \\
& \prod_{i=1}^{n} t_{i}^{\alpha-1}\left(1-\exp \left\{-\left(\frac{t_{i}}{\sigma}\right)^{\alpha}\right\}\right)^{\theta-1},
\end{aligned}
$$

onde $\alpha>0, \theta>0, \sigma>0$ e $D$ denota o conjunto de dados observados sobre os tempos de falha.

Para obtermos uma amostra a posteriori das densidades marginais $\alpha, \theta$ e $\sigma$, fazemos uso do algoritmo Gibbs Sampling que baseia-se em sucessivas gerações das distribuições condicionais a posteriori de $\pi(\alpha \mid \theta, \sigma, D), \pi(\theta \mid \alpha, \sigma, D)$ e $\pi(\sigma \mid \alpha, \theta, D)$.

Da densidade a posteriori conjunta dada em (4.15) pode-se mostrar que as densidades condicionais a posteriori para o algoritmo de Gibbs são dadas por

$$
\begin{aligned}
& \pi(\alpha \mid \theta, \sigma, D) \propto \alpha^{n+a_{1}-1} \exp \left\{-\sum_{i=1}^{n}\left(\frac{t_{i}}{\sigma}\right)^{\alpha}-b_{1} \alpha\right\} \prod_{i=1}^{n} t_{i}^{\alpha-1}\left(1-\exp \left\{-\left(\frac{t_{i}}{\sigma}\right)^{\alpha}\right\}\right)^{\theta-1}, \\
& \pi(\theta \mid \alpha, \sigma, D) \propto \theta^{n+a_{2}-1} e^{-b_{2} \theta} \prod_{i=1}^{n} t_{i}^{\alpha-1}\left(1-\exp \left\{-\left(\frac{t_{i}}{\sigma}\right)^{\alpha}\right\}\right)^{\theta-1}, \\
& \pi(\sigma \mid \alpha, \theta, D) \propto \theta^{-n \sigma+a_{3}-1} \exp \left\{-\sum_{i=1}^{n}\left(\frac{t_{i}}{\sigma}\right)^{\alpha}-b_{3} \sigma\right\} \prod_{i=1}^{n} t_{i}^{\alpha-1}\left(1-\exp \left\{-\left(\frac{t_{i}}{\sigma}\right)^{\alpha}\right\}\right)^{\theta-1} .
\end{aligned}
$$

Observe que as densidades dadas em (4.16) não são conhecidos de modo que faremos uso do algoritmo de Metropolis-Hastings para gerar as variáveis $\alpha, \theta$ e $\sigma$.

\subsubsection{O modelo IDB}

A função de verossimilhança de $\delta, \beta$ and $\theta$ é dada por,

$$
L_{n}(\delta, \beta, \theta)=\exp \left\{-\frac{\delta \sum_{i=1}^{n} t_{i}^{2}}{2}\right\} \prod_{i=1}^{n} \frac{(1+\beta t) \delta t_{i}+\theta}{\left(1+\beta t_{i}\right)^{\theta / \beta+1}} .
$$

Assumindo independência entre os parametros, consideremos a seguintes densidades a priori para $\delta, \beta$ e $\theta$ :

$$
\delta \sim \Gamma\left(c_{1}, d_{1}\right) ; \operatorname{com} c_{1} \text { e } d_{1} \text { conhecidos; }
$$




$$
\begin{aligned}
\beta & \sim \Gamma\left(c_{2}, d_{2}\right) ; \text { com } c_{2} \text { e } d_{2} \text { conhecidos; } \\
\theta & \sim \Gamma\left(c_{3}, d_{3}\right) ; \text { com } c_{3} \text { e } d_{3} \text { conhecidos. }
\end{aligned}
$$

Considerando (4.17)-(4.18) e o teorema de Bayes pode-se mostrar que a densidade conjunta a posteriori de $\delta, \beta$ and $\theta$ é dada por,

$$
\pi(\alpha, \theta, \sigma \mid D) \propto \delta^{c_{1}-1} \beta^{c_{2}-1} \theta^{c_{3}-1} \exp \left\{-d_{1} \delta-\frac{\delta \sum_{i=1}^{n} t_{i}^{2}}{2}-d_{2} \beta-d_{3} \theta\right\} \Psi_{n}(\delta, \beta, \theta),
$$

onde $\Psi_{n}(\delta, \beta, \theta)=\prod_{i=1}^{n} \frac{(1+\beta t) \delta t_{i}+\theta}{\left(1+\beta t_{i}\right)^{\theta / \beta+1}}$.

De (4.19) pode-se mostrar que as densidades condicionais requeridas para o algoritmo de Gibbs são dadas por

$$
\begin{aligned}
& \pi(\delta \mid \beta, \theta, D) \propto \delta^{c_{1}-1} \exp \left\{-d_{1} \delta-\frac{\delta \sum_{i=1}^{n} t_{i}^{2}}{2}\right\} \Psi_{n}(\delta, \beta, \theta), \\
& \pi(\beta \mid \delta, \theta, D) \propto \beta^{c_{2}-1} e^{-d_{2} \beta} \Psi_{n}(\delta, \beta, \theta), \\
& \pi(\theta \mid \delta, \beta, D) \propto \theta^{a_{3}-1} e^{-d_{3} \theta} \Psi_{n}(\delta, \beta, \theta) .
\end{aligned}
$$

Observamos que as densidades condicionas dadas em (4.20) não são padrão, e portanto usamos o algoritmo de Metropolis-Hasting para gerar as variáveis $\delta, \beta$ e $\theta$ dentro do ciclo do amostrador de Gibbs.

\subsubsection{O modelo exponencial-potência}

De (4.7), a função de verossimilhança para $\alpha$ e $\beta$ é dada por

$$
L(\alpha, \beta)=\beta^{n} \alpha^{-n \beta} \prod_{i=1}^{n} t_{i}^{\beta-1} \exp \left\{n+\sum_{i=1}^{n}\left(\frac{t_{i}}{\alpha}\right)^{\beta}-\sum_{i=1}^{n} e^{\left(\frac{t_{i}}{\alpha}\right)^{\beta}}\right\} .
$$

Assumindo a densidades a priori,

$$
\begin{aligned}
& \alpha \sim \Gamma\left(e_{1}, f_{1}\right) \text { com } e_{1} \text { e } f_{1} \text { conhecidos, } \\
& \beta \sim \Gamma\left(e_{2}, f_{2}\right) \text { com } e_{2} \text { e } f_{2} \text { conhecidos. }
\end{aligned}
$$


De (4.21)-(4.22) e do teorema de Bayes pode-se mostrar que a densidade a posteriori de $\alpha$ e $\beta$ é dada por,

$$
\pi(\alpha, \beta \mid D) \propto \beta^{n+\epsilon_{2}-1} \alpha^{-n \beta+e_{1}-1} \prod_{i=1}^{n} t_{i}^{\beta-1} \exp \left\{\sum_{i=1}^{n}\left(\frac{t_{i}}{\alpha}\right)^{\beta}-\sum_{i=1}^{n} e^{\left(\frac{t_{i}}{\alpha}\right)^{\beta}}-f_{1} \alpha-f_{2} \beta\right\},
$$

onde $\alpha>0$ e $\beta>0$.

De (4.23), obtemos a densidade condicional a posteriori para o algoritmo de Gibbs que é dada por

$$
\begin{aligned}
& \pi(\alpha \mid \beta, D) \propto \alpha^{-n \beta+e_{1}-1} \exp \left\{\sum_{i=1}^{n}\left(\frac{t_{i}}{\alpha}\right)^{\beta}-\sum_{i=1}^{n} e^{\left(\frac{t_{i}}{\alpha}\right)^{\beta}}-f_{1} \alpha\right\}, \\
& \pi(\beta \mid \alpha, D) \propto \beta^{n+e_{2}-1} \prod_{i=1}^{n} t_{i}^{\beta-1} \exp \left\{\sum_{i=1}^{n}\left(\frac{t_{i}}{\alpha}\right)^{\beta}-\sum_{i=1}^{n} e^{\left(\frac{t_{i}}{\alpha}\right)^{\beta}}-f_{2} \beta\right\} .
\end{aligned}
$$

De (4.24) observamos que as densidades condicionais a posteriori não são conhecidas pelo que faremos uso do algoritmo de Metropolis-Hasting para gerar as variáveis $\alpha$ e $\beta$ dentro do ciclo do algoritmo de Gibbs.

\subsubsection{Modelo de mistura distribuições de Gamas}

A função de verossimilhança para mistura de distribuição de gamas em (4.10) (veja, Dempster et al. 1977) é dada por,

$$
L\left(\alpha_{1}, \alpha_{2}, \beta, p\right)=\prod_{i=1}^{n}\left[p f\left(t_{i} ; \alpha_{1}, \beta\right)\right]^{z_{i 1}}\left[(1-p) f\left(t_{i} ; \alpha_{2}, \beta\right)\right]^{1-z_{i 1}}
$$

onde $z_{i 1}$ é uma função indicadora $\left(z_{i 1}=1(0)\right.$ se $t_{i}$ pertenca à primeira (segunda) componente do modelo de mistura).

Assumindo as seguintes densidades a priori independentes:

$$
\begin{aligned}
p & \sim \operatorname{Beta}\left(\gamma_{1}, \gamma_{2}\right), \text { com } \gamma_{1} \text { e } \gamma_{2} \text { conhecidos, } \\
\alpha_{j} & \sim \Gamma\left(1, \lambda_{j}\right), \quad \text { com } \lambda_{j} \text { conhecidos } j=1,2, \\
\beta & \sim \Gamma\left(\beta_{1}, \beta_{2}\right), \quad \beta_{1} \text { e } \beta_{2} \text { conhecidos }
\end{aligned}
$$


onde $\operatorname{Beta}\left(\delta_{1}, \delta_{2}\right)$ denota uma distribuição Beta com parâmetros $\delta_{1}$ and $\delta_{2}$. Assim, a densidade conjunta a posteriori $\alpha_{1}, \alpha_{2}, \beta$ e $p$ é dada por,

$$
\begin{aligned}
\pi\left(\alpha_{1}, \alpha_{2}, \beta, P \mid D, \underline{z}\right) \propto & p^{z .1+\gamma_{1}-1}(1-p)^{n-z_{1}+\gamma_{2}-1} \beta^{\alpha_{1} z_{.1}+\alpha_{2}\left(n-z_{1}\right)+\beta_{1}-1} \\
& \times \exp \left\{-\beta\left(\sum_{i=1}^{n} t_{i}+\beta_{2}\right)-\lambda_{1} \alpha_{1}-\lambda_{2} \alpha_{2}\right\} \frac{\left(\prod_{i=1}^{n} t_{i}^{z_{i 1}}\right)^{\alpha_{1}}}{\left[\Gamma\left(\alpha_{1}\right)\right]^{z_{11}}} \\
& \times \frac{\left(\prod_{i=1}^{n} t_{i}^{1-z_{i 1}}\right)^{\alpha_{2}}}{\left[\Gamma\left(\alpha_{1}\right)\right]^{n-z_{1}}},
\end{aligned}
$$

onde $z_{.1}=\sum_{i=1}^{n} z_{i 1}$.

As densidades condicionais a posteriori para o algoritmo de Gibbs são dadas por:

$$
\begin{aligned}
\pi\left(z_{i 1} \mid \alpha_{1}, \alpha_{2}, \beta, D\right) & \sim \operatorname{bernoulli}\left(\frac{p f\left(t_{i} ; \alpha_{1}, \beta\right)}{p f\left(t_{i} ; \alpha_{1}, \beta\right)+(1-p) f\left(t_{i} ; \alpha_{2}, \beta\right)}\right) ; \\
\pi\left(p \mid \alpha_{1}, \alpha_{2}, \beta, \underline{z}, D\right) & \sim \operatorname{Beta}\left(z_{.1}+\gamma_{1}, n-z_{.1}+\gamma_{2}\right) \\
\pi\left(\beta \mid \alpha_{1}, \alpha_{2}, p, \underline{z}, D\right) & \sim \Gamma\left(\alpha_{1} z_{.1}+\alpha_{2}\left(n-z_{.1}\right)+\beta_{1}, \beta_{2}+\sum_{i=1}^{n} t_{i}\right) ; \\
\pi\left(\alpha_{1} \mid \alpha_{2}, \beta, p, \underline{z}, D\right) & \propto \exp \left\{-\lambda_{1} \alpha_{1}\right\} \frac{\beta^{\alpha_{1} z_{.1}}\left(\prod_{i=1}^{n} t_{i}^{z_{i 1}}\right)^{\alpha_{1}}}{\left[\Gamma\left(\alpha_{1}\right)\right]^{z .1}} ; \\
\pi\left(\alpha_{2} \mid \alpha_{1}, \beta, p, \underline{z}, D\right) & \propto \exp \left\{-\lambda_{2} \alpha_{2}\right\} \frac{\beta^{\alpha_{2}\left(n-z_{1}\right)}\left(\prod_{i=1}^{n} t_{i}^{1-z_{i 1}}\right)^{\alpha_{2}}}{\left[\Gamma\left(\alpha_{2}\right)\right]^{n-z_{.1}}} .
\end{aligned}
$$

Note que as duas últimas densidades condicionais requer do algoritmo Metropolis-Hasting no passo da implementação da metodologia $\mathrm{MCMC}$.

\subsection{Discriminação de modelos}

$\mathrm{Na}$ seção 4.2 foram apresentados vários modelos para tempos de vida. Surge então a necessidade de determinar qual ou quais modelos são mais apropriadas para os tempos de vida com função de taxas de falha do tipo "bathtub". 
Os procedimentos descritos no capítulo 1, são adotados para verificarmos qual é o melhor modelo dentre os modelos estudados. Por exemplo, para o modelo Weibullexponenciada a estimativa de Monte Carlo da densidade preditiva de $t_{r}$ dado $D_{(r)}(\mathrm{CPO})$ com as amostras Gibbs é dada por

$\hat{f}\left(t_{r} \mid D_{(r)}\right)^{-1}=\frac{2}{R S} \sum_{r=1}^{R} \sum_{s=\frac{s}{2}+1}^{S} \frac{\left(\sigma^{(r, s)}\right)^{\alpha^{(r, s)}}}{\alpha^{(r, s)} \theta^{(r, s)}\left[1-\exp \left(-\left(\frac{t_{r}}{\sigma^{(r, s)}}\right)^{\alpha^{(r, s)}}\right)\right]^{\theta^{(r, s)}-1} \exp \left(-\left(\frac{t_{r}}{\sigma^{(r, s)}}\right)^{\alpha^{(r, s)}}\right)\left(t_{r}\right)^{\alpha^{(r, s)}-1}}$.

sendo $D_{(r)}=\left(t_{1}, \ldots, t_{r-1}, t_{r+1}, \ldots, t_{n}\right), r=1, \ldots, n, \alpha^{(r, s)}, \theta^{(r, s)}$ e $\sigma^{(r, s)}$ são gerados de (4.15) considerando $S$ iterações para cada uma das $R$ cadeias considerando diferentes pontos iniciais para $\alpha, \theta$ e $\sigma$.

Da mesma forma, pode-se obter as estimativas dos CPO's para os modelos IDB. exponencial-potência e mistura de distribuições gammas.

Usaremos a estimativa do pseudo-fator de Bayes dada em (1.11), computada com as estimativas dos CPO's para discriminação entre os modelos considerados neste Capítulo.

\subsection{Alguns exemplos}

\subsubsection{Exemplo 1}

Para ilustrar a metodologia apresentada nas seções anteriores consideramos o conjunto de dados ilustradas em Arset (1987). Os dados descrevem os tempos de vida de 50 projetos industriais.

\section{Modelo Weibull-exponenciada}

Para análise dos dados da tabela (4.1), primeramente assume-se a distribuição Weibullexponenciada (4.1). Considerando a densidade a priori $\alpha, \theta$ e $\sigma$ dada em (4.14) com 
Tabela 4.1: Tempos de sobrevivência de 50 projetos

\begin{tabular}{lllllllllllllllllll}
\hline \hline 0.1 & 0.2 & 1 & 1 & 1 & 1 & 1 & 2 & 3 & 6 & 7 & 11 & 12 & 18 & 18 & 18 & 18 & 18 & 21 \\
32 & 36 & 40 & 45 & 46 & 47 & 50 & 55 & 60 & 63 & 63 & 67 & 67 & 67 & 67 & 72 & 75 & 79 & 82 \\
82 & 83 & 84 & 84 & 84 & 85 & 85 & 85 & 85 & 85 & 86 & 86 & & & & & & & \\
\hline \hline
\end{tabular}

$a_{1}=8,6 . b_{1}=1,83, a_{2}=7,58, b_{2}=51,98 . a_{3}=26491,6$ and $b_{3}=290,251$ (a escolha desses valores para os parâmetros da densidade a priori, foram baseadas numa opinião experinte combinada com a análise preliminar dos dados), geramos 10 cadeias cada uma com 5.000 iterações e monitoramos a convergência das amostras Gibbs usando o método de Gelman e Rubin (1992) que utiliza a técnica de análise de variância para determinar se mais iterações são necessarias. Para cada parâmetro e em cada cadeia consideramos as iterações 2.525 - ésima, 2.550 - ésima, . . , 5.000 - ésima a qual produz uma amostra de Gibbs de tamanho 1.000. Na tabela 4.2, reportamos o resumo a posteriori dos parâmetros do modelo e, na figura 4.1, temos as densidades marginais a posteriori aproximadas obtidas considerando as 1.000 amostras de Gibbs. Também temos na tabela 4.2, o resultado da estimativa dos fatores de redução de escala potêncial $\hat{R}$ (veja, Gelmann e Rubin, 1992) para todos os parâmetros. Neste caso o número de iterações foi suficiente para a convergencia $((\sqrt{\hat{R}}<1.1)$, para todos os parâmetros $)$.

Tabela 4.2: Sumário a posteriori para o model Weibull-exponenciada

\begin{tabular}{|llllcc|}
\hline \hline & Média & Mediana & D.P. & $95 \%$ intervalo de credibilidade & $\hat{R}$ \\
\hline$\alpha$ & 2,6444 & 2,6446 & 0,27758 & $(2,081139 ; 3,16199)$ & 1,002173 \\
$\theta$ & 0,20422 & 0,20432 & 0,05406 & $(0,10314 ; 0,317079)$ & 1,002107 \\
$\sigma$ & 91,016 & 91,005 & 0,547375 & $(89,9555 ; 92,0941)$ & 1,0028216 \\
\hline \hline
\end{tabular}




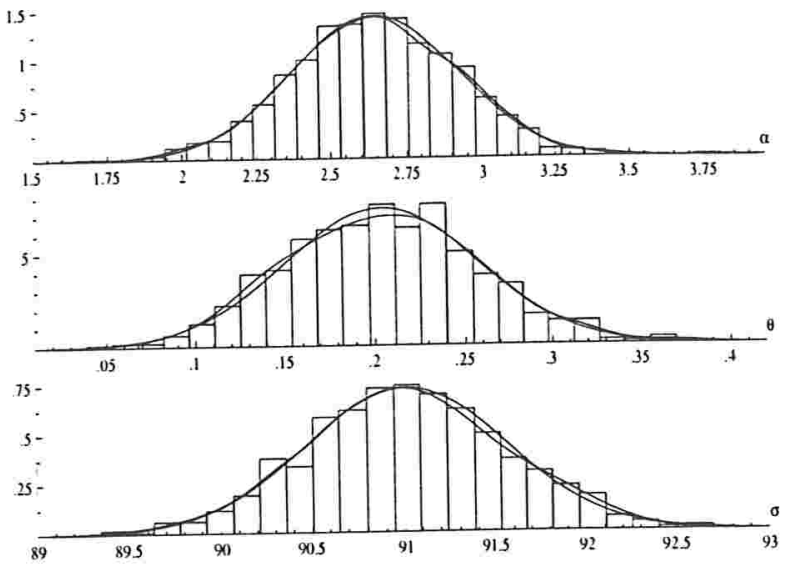

Figura 4.1: Densidades marginais a posteriori aproximadas de $\alpha, \theta$ e $\sigma$ parâmetros do modelo Weibull-exponenciada

\section{Modelo IDB}

Considerando o modelo IBD (4.4) e a densidade a priori dada em (4.19) com $c_{1}=33,30$, $d_{1}=73669,89, c_{2}=0,8775, d_{2}=0,2944, c_{3}=1,4832$ and $d_{3}=7,315$, geramos 20 cadeias separadas cada uma com 2.000 iterações e consideramos para cada parâmetro as iterações 1.020 - ésima, . ., 2.000 - ésima, de modo que as 20 cadeias fornecem uma amostra de tamanho 1.000. Na tabela 4.3, obtemos o resumo a posteriori dos parâmetros do modelo e, na figura 4.2 , temos as densidades marginais a posteriori aproximados para $\delta, \beta$ e $\theta$ considerando as $S=1.000$ amostras Gibbs. Também temos na tabela 4.3, a estimativa dos fatores de redução de escala potêncial $\hat{R}$ para todos os parâmetros, a qual indica que as cadeias convergiram.

\section{Modelo exponencial-potência}

Consideramos agora, o modelo exponencial-potência (4.7) e a densidade a priori (4.24) com $e_{1}=113.33, f_{1}=1.07, e_{2}=28.58$ e $f_{2}=53.4$, geramos 8 cadeias separadas 
Tabela 4.3: Sumário a posteriori dos parâmetros do modelo IDB

\begin{tabular}{|clllcc|}
\hline \hline & Média & Mediana & D.P. & 95\% Intervalo de credibilidade & $\hat{R}$ \\
\hline$\delta$ & 0,00030 & 0,00030 & 0,00005 & $(0,000229 ; 0,000407)$ & 1,006153 \\
$\beta$ & 0,72470 & 0,5024 & 0,8999 & $(0,0271 ; 2.527)$ & 1,0000402 \\
6 & 0,18837 & 0,16352 & 0,12462 & $(0,0247 ; 0,48762)$ & 1,0002546 \\
\hline
\end{tabular}

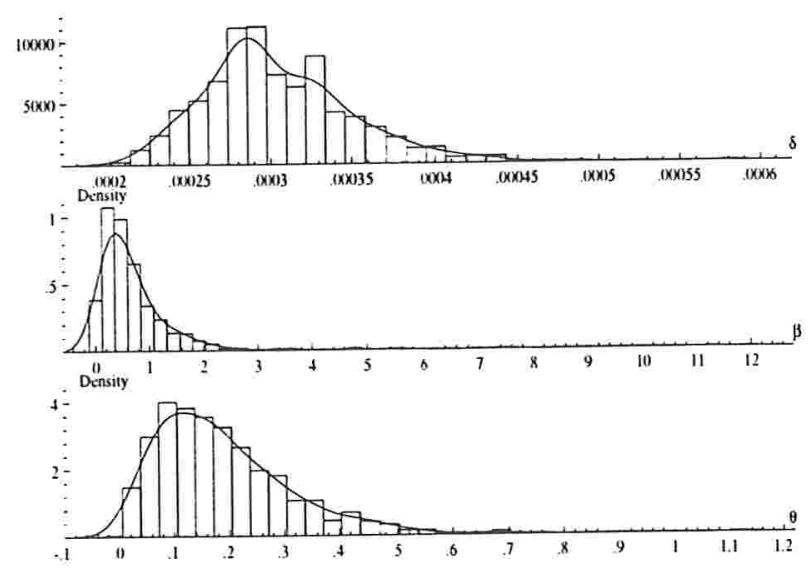

Figura 4.2: Densidades marginais a posteriori aproximadas de $\delta, \beta$ e $\theta$ parâmetros do modelo IDB

cada um com 50.000 iterações, foram consideradas as iterações 25.025 - ésima, 25.050 ésima, . ., 50.000 - ésima que produz uma amostra Gibbs de tamanho 8.000. Na tabela 4.4, obtemos o sumário a posteriori dos parâmetros do modelo e, na figura 4.3, temos a densidades marginais a posterioris aproximados considerando os $S=8.000$ amostras Gibbs. Para todos os parâmetros, observamos (veja tabela 4.4), $\sqrt{\hat{R}}<1,1$, indicando a convergência. 
Tabela 4.4: Sumário a posteriori dos parâmetros do modelo exponencial-potência

\begin{tabular}{|c|c|c|c|c|c|}
\hline & Média & Mediana & D.P. & $95 \%$ Intervalo de credibilidade & $\hat{R}$ \\
\hline$\alpha$ & 76,498 & 76,4919 & 2,991 & $(68,8193 ; 81,3473)$ & 1,000109 \\
\hline$\beta$ & 2,166 & 2,166 & 0,315 & $(1,18404 ; 2,64272)$ & 1,009575 \\
\hline
\end{tabular}

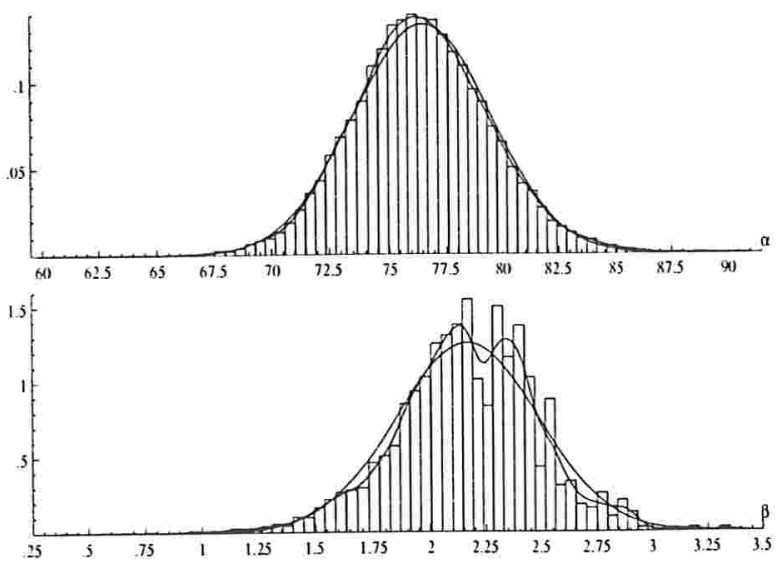

Figura 4.3: Densidades marginais a posteriori aproximadas de $\alpha$ e $\beta$ parâmetros do modelo exponencial-potência

\section{Modelo de Mistura}

Finalmente considerando, a mistura de gamas dado em (4.10), e a densidade a priori (4.26) com $\gamma_{1}=0,5, \gamma=0,5, \lambda_{j}=0,000001(j=1,2), \beta_{1}=1$ e $\beta_{2}=0,00002$, geramos 20 cadeais separadas de Gibbs cada uma com 5.000 iterações. Para cada parâmetro em cada cadeia consideramos as itearações 2.550 - ésima, 2.600 - ésima, . ., 5.000 - ésima produzindo uma amostra Gibbs de tamanho 1.000. Na tabela 4.5, reportamos as quantidades de interesse, média, mediana, desvio padrão (D.P.), intervalos de credibilidade de $95 \%$ e as estimativas dos fatores de redução de escala potêncial $\hat{R}$ para cada um dos parâmetros. 
Tabela 4.5: Sumário a posteriori dos parâmetros do modelo de mistura de distribuições gama

\begin{tabular}{|clllccc|}
\hline \hline & Média & Mediana & D.P. & 95\% & Intervalo de credibilidade & $\hat{R}$ \\
\hline$\alpha_{1}$ & 14,542 & 12,494 & 10,405 & $(1,33710 ; 39,6366)$ & 1,000312 \\
$\alpha_{2}$ & 0,91499 & 0,89006 & 1,19612 & $(0,68565 ; 1,32152)$ & 1,000304 \\
$\beta$ & 0,02148 & 0,02030 & 0,00655 & $(0,01271 ; 0,04058)$ & 1,085947 \\
$p$ & 0,02672 & 0,00601 & 0,09164 & $(0,000012 ; 0,0318169)$ & 1,021205 \\
\hline
\end{tabular}

Observamos na tabela 4.5 , que $\sqrt{\hat{R}}<1,1$ para todos os parâmetros o que nos indica a convergência das amostras geradas. Na figura 4.4, representamos a densidades marginais a posteriori aproximadas com as 1.000 amostras Gibbs para os parâmetros do modelo de mistura de distribuições gama.

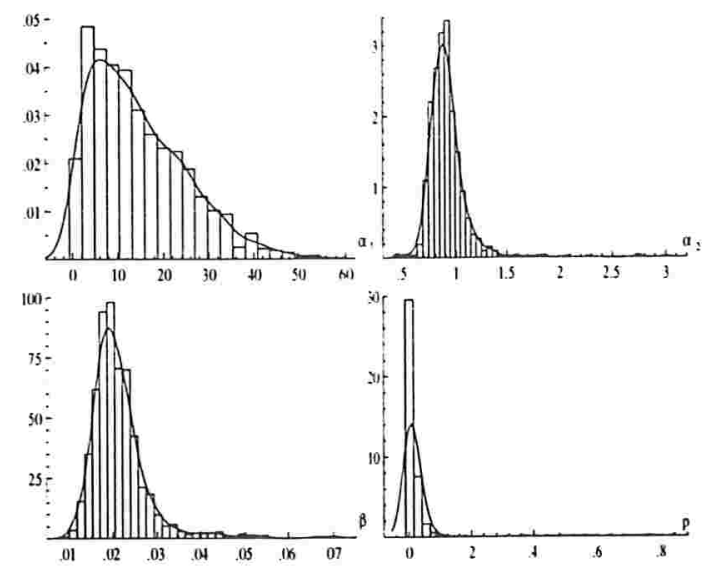

Figura 4.4: Densidades marginais a posteriori aproximados de $\alpha_{1}, \alpha_{2}, \beta$ e $p$ parâmetros do modelo de mistura de distribuições gama 


\section{Descrimminação dos modelos}

Para selecionar entre os modelos Weibull-exponenciada (4.1), IBD dada em (4.5), mistura de distribuições gama dada em (4.10) e exponencial-potência dada em (4.7), se ajusta melhor aos dados da tabela 4.1, utilizamos a técnica das densidades preditivas condicionais ordenadas (CPO) para o computo do pseudo-fator Bayes e o método gráfico de seleção de modelos (veja capítulo 1).

Com as amostras Gibbs geradas, temos na tabela 4.6, o pseudo-fator de Bayes (1.11) para a seleção do melhor modelo para os dados de tempo de vida dados na tabela 4.1.

Na figura 4.5, temos o gráfico da razão dos CPO para cada modelo com respeito aos outros versus as observações. Valores da razão de CPO maior que um indicam a preferência do primeiro modelo. Por exemplo, na figura 4.5, o "plot" do modelo Weibullexponenciada versus o modelo exponencial-pontência indica que 28 das 50 observações sustentam o modelo Weibull-exponenciada sobre o modelo exponencial-potência. Similarmente, 38 observações sustentam o modelo Weibull-exponenciada sobre o modelo IDB, 33 observações sustentam ao modelo Weibull-exponenciada sobre o modelo de mistura de distribuições gamas, 38 observações sustentam o modelo IDB sobre o modelo exponencialpotência, 32 observações sustentam o modelo exponencial-potência sobre o modelo de mistura de gamas, e 39 observações sustentam ao modelo de mistura de gamas sobre o modelo IDB. Assim, em conclusão, esse critério e o método do pseudo-fator de Bayes mostram que o modelo Weibull-exponenciada é um dos que se ajustam melhor aos dados da tabela 4.1 . 
Tabela 4.6: Pseudo-Fator de Bayes

\begin{tabular}{|lc|}
\hline \hline Modelos & PSFB \\
\hline Weibull-exponenciada vs exponencial-potência & 32,83 \\
Weibull-exponenciadal vs IDB & 17,61 \\
Weibull-exponenciada vs mistura de gammas & 620,75 \\
exponencial-potência vs IDB & 0,54 \\
exponencial-potência vs mistura gamas & 18,91 \\
IDB vs mistura de gamas & 35,26 \\
\hline
\end{tabular}
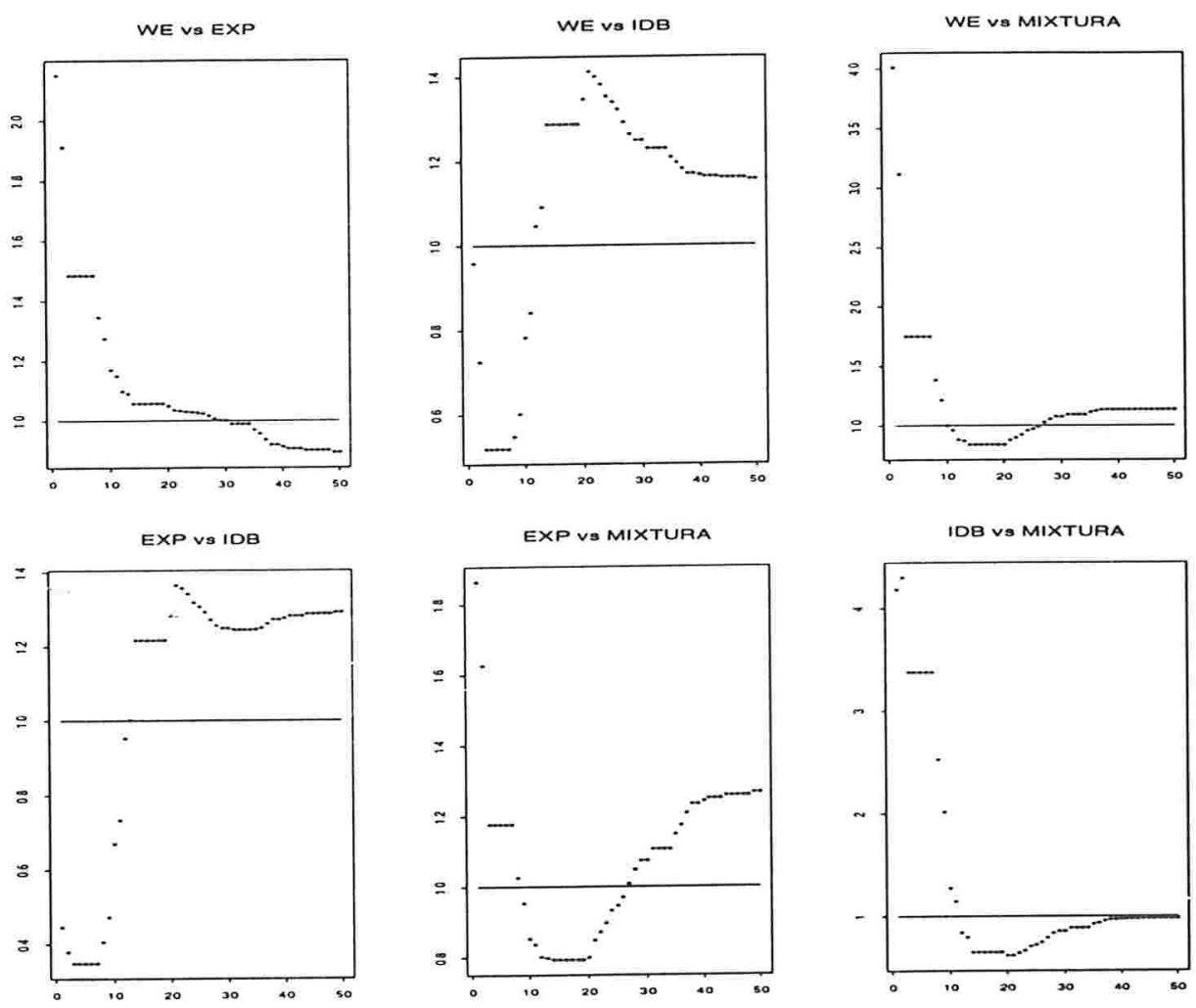

Figura 4.5: Gráfico da razão dos CPO para os diferentes modelos versus observações,

\subsubsection{Exemplo 2}

Na tabela 4.7, temos 50 observações geradas da densidade (5.1) com parâmetros $\alpha=2$, $\theta=0,3$ e $\sigma=1$, não incluindo dados censurados. 
Tabela 4.7: Dados gerados da densidade (4.1)

\begin{tabular}{llllllllll}
\hline \hline 0,17087 & 0,67164 & 0,044448 & 1,0409 & 0,14450 & 0,44826 & 0,77785 & 0,0048173 & 0,0032595 & 0,27430 \\
0,0015956 & 0,48105 & 0,66123 & 1,8675 & 0,22152 & 0,63347 & 0,012058 & 0,41785 & 1,9351 & 0,57678 \\
0,67180 & 0,15366 & 1,0742 & 0,46588 & 0,038705 & 0,72372 & 0,17963 & 1,4175 & 0,48098 & 0,36706 \\
0,64136 & 0,13995 & 1,4367 & 0,34256 & 0,29463 & 0,27305 & 0,060425 & 0,15204 & 1,0947 & 0,53263 \\
1,2511 & 0,43501 & 0,30608 & 0,40391 & 0,025192 & 0,39053 & 0,42179 & 1,0422 & 0,0048170 & 1,3440 \\
\hline \hline
\end{tabular}

\section{Modelo Weibull-exponenciada}

Considerando o modelo Weibull-exponenciada (4.1), a a densidade a priori (4.14) com $a_{1}=0,4, b_{1}=0,2, a_{2}=0,6, b_{2}=0,18, a_{3}=0,001 b_{3}=0,001$, geramos 20 cadeias separadas cada uma com 2.000 iterações e, monitoramos a convergência usando o método de Gelman e Rubin (1992). Para cada parâmetro consideramos as 1.010 - ésima, 1.020 ésima, .., 2.000-ésima as 20 cadeias produz uma amostra de Gibbs de tamanho 2.000. Na tabela 4.8 , reportamos a sumário a posterior dos parâmetros e, na figura 4.6 temos a densidade marginal a posteriori aproximado considerando as 2.000 amostras Gibbs. Também na tabela 4.8, temos a estimativa dos fatores de redução de escala $\hat{R}$ (veja Gelman e Rubin, 1992) para todos prâmetros. Neste caso, o número de iteraçǒes consideradas foram suficientes para atingir a convergência uma vez que $(\sqrt{\hat{R}}<1.1)$ para todos parâmetros.

Tabela 4.8: Sumário a posteriori dos parâmetros do modelo Weibull-exponenciada

\begin{tabular}{|ccllcc|}
\hline \hline & Média & Mediana & D.P. & $95 \%$ Intervalo de Credibilidade & $\hat{R}$ \\
\hline$\alpha$ & 1,7279 & 1,7054 & 0,3570 & $(1,0938 ; 2,4497)$ & 1,000382 \\
$\theta$ & 0,4098 & 0,3996 & 0,12686 & $(0,1822 ; 0,6866)$ & 1,001075 \\
$\sigma$ & 0,9944 & 0,9889 & 0,1753 & $(0,6783 ; 1,3637)$ & 1,001018 \\
\hline \hline
\end{tabular}



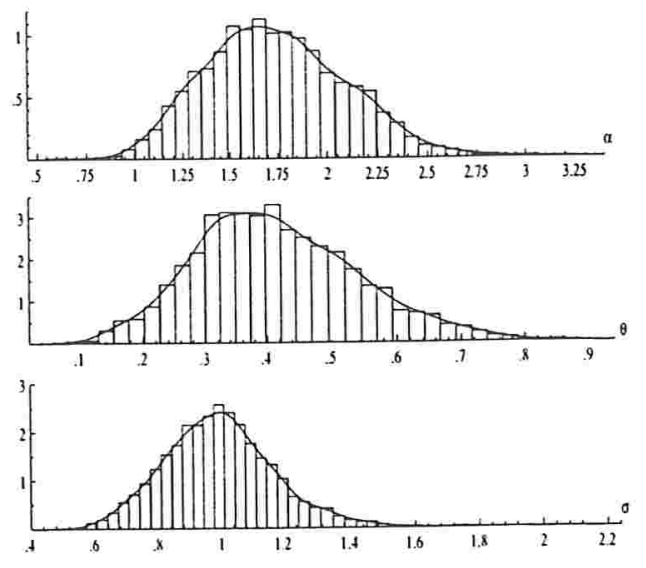

Figura 4.6: Densidades marginais a posteriori aproximado de $\alpha, \theta$ e $\sigma$ parâmetros do modelo Weibull-exponenciada

\section{Modelo IBD}

Considerando o modelo IBD (4.4) e a densidade a priori (4.19) $\operatorname{com} c_{1}=7,76, d_{1}=3,94$, $c_{2}=34,68, d_{2}=2,63, c_{3}=6,75$ e $d_{3}=1,5$, geramos 20 cadeias separadas de Gibbs cada um com 2.000 iterações. Para cada parâmetro consideramos as iterações 1.010 ésima, 1.020-ésima, ..., 2.000-ésima, totalizando assim uma amostra Gibbs de tamanho 2.000. Na tabela 4.9, apresentamos o sumário a posteriori para os parâmetros do modelo e, na figura 4.7 , temos as densidades marginais a posteriori aproximadas considerando as 2.000 amostras Gibbs. Também temos na tabela 4.9, as estimativas dos fatores de redução de escala potêncial $\hat{R}$ para todos os parâmetros, o qual indica a convergência das cadeias.

\section{Modelo exponencial-potência}

Considerando agora, o modelo exponencial-potência (4.7) a densidade a priori (4.24) com $e_{1}=4,58, f_{1}=4,78, e_{2}=4,161$ e $f_{2}=6,451$, geramos 20 cadeias separadas de Gibbs cada uma com 5.000 iterações e consideramos as iterações 2.525 - ésima, 2.550 - 
Tabela 4.9: Sumário a posteriori dos parâmetros do modelo IDB

\begin{tabular}{|ccllccc|}
\hline \hline & Média & Mediana & D.P. & $95 \%$ & Intervalo de Credibilidade & $\hat{R}$ \\
\hline$\delta$ & 2,0098 & 1,9954 & 0,4110 & $(1,24363 ; 2,8898)$ & 1,000107 \\
$\beta$ & 13,283 & 13,197 & 2,247 & $(9,3060 ; 18,2471)$ & 1,0000402 \\
$\theta$ & 3,8369 & 3,7369 & 0,9027 & $(2,3219 ; 5,8996)$ & 1,007290 \\
\hline
\end{tabular}
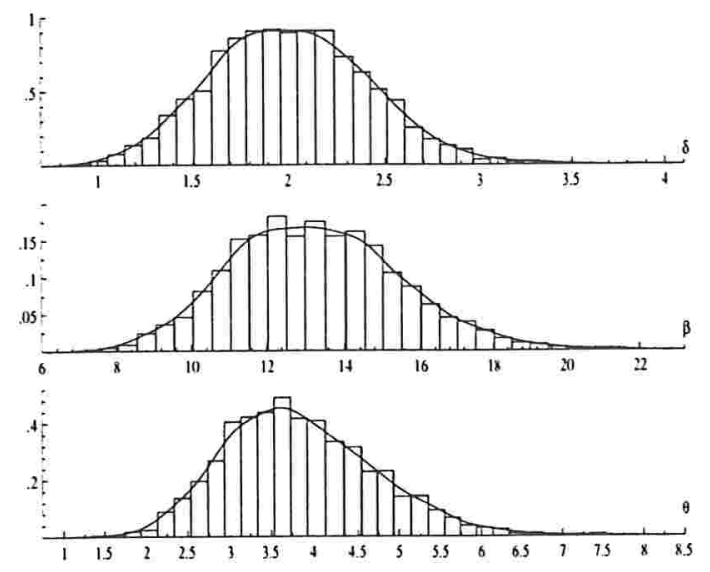

Figura 4.7: Densidades marginais a posteriori aproximada de $\delta, \beta$ e $\theta$ parâmetros do modelo IDB

ésima, . ., 5.000 -ésima, a qual para as 20 cadeias produz uma amostra de tamanho 2.000. Na tabela 4.10 apresentamos o sumário a posteriori dos parâmetros do modelo e na figura 4.8 temos a densidade marginal a posteriori aproximado com as $S=2.000$ amostras Gibbs. Para todos parâmetros observamos (ver tabela 4.10), $\sqrt{\hat{R}}<1.1$, indicando a convergência. 
Tabela 4.10: Sumário a posteriori dos parâmetros do modelo exponencial-potência

\begin{tabular}{|clllccc|}
\hline \hline & Média & Mediana & D.P & $95 \%$ & Intervalo de Credibilidade & $\hat{R}$ \\
\hline$\alpha$ & 0,9828 & 0.9674 & 0,16199 & $(0,7169 ; 1,3393)$ & 1,000081 \\
$\beta$ & 0,6575 & 0.6419 & 0,20657 & $(0,2969 ; 1,1467)$ & 1,0005173 \\
\hline \hline
\end{tabular}

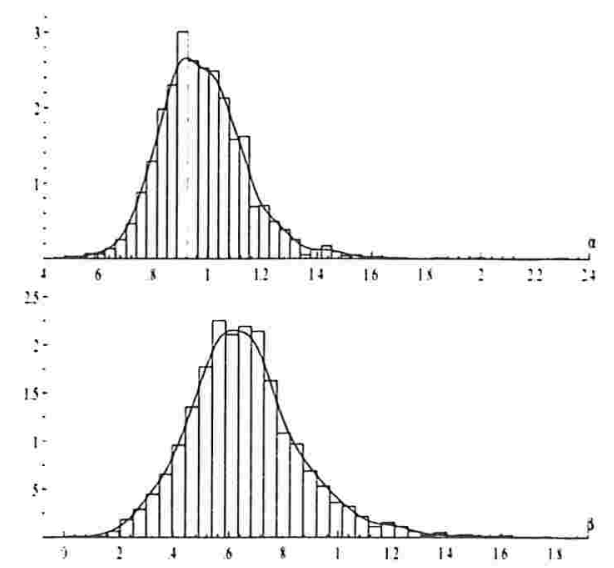

Figura 4.8: Densidades marginais a posteriori aproximadas de $\alpha$ e $\beta$ do modelo exponencial-potência

\section{Modelo de mistura de gamas}

Finalmente considerando, a mistura de distribuições gamas dada em (4.10), e a densidade a priori (4.26) com $\gamma_{1}=1, \gamma_{2}=1, \lambda_{j}=0,000001(j=1,2), \beta_{1}=1 \beta_{2}=0,00001$, geramos 20 cadeias separadas de Gibbs cada um com 5.000 iterações. para cada parâmetro consideramos as iterações 2.550 - ésima, 2.600 - ésima, . ., 5.000 - ésima, as 20 cadeias produz uma amostra de tamanho 1.000. Na tabela 4.11, reportamos o sumário a posteriori dos parâmetros do modelo e, na figura 4.9, temos a densidades marginais a posteriori aproximadas pelas 1.000 amostras Gibbs. Também obtemos a convergência, já que, a estimativa dos fatores de redução de escala potencial $\hat{R} \approx 1$ para todos os parâmetros. 
Tabela 4.11: Sumário a posteriori dos parâmetros do modelo de mistura de distribuições gamas

\begin{tabular}{|ccllccc|}
\hline \hline & Média & Mediana & D.P. & $95 \%$ & Intervalo de Credibilidade & $\hat{R}$ \\
\hline$\alpha_{1}$ & 3,5629 & 2,9961 & 3,3662 & $(0,2050 ; 10,0671)$ & 1,000312 \\
$\alpha_{2}$ & 0,4548 & 0,4416 & 0,0958 & $(0,3412 ; 0,6667)$ & 1,000304 \\
$\beta$ & 2,1423 & 2,2023 & 0,1023 & $(1,2383 ; 4,1721)$ & 1,085947 \\
$p$ & 0,0308 & 0,0054 & 0,10229 & $(0,000013 ; 0,4247)$ & 1,021205 \\
\hline \hline
\end{tabular}
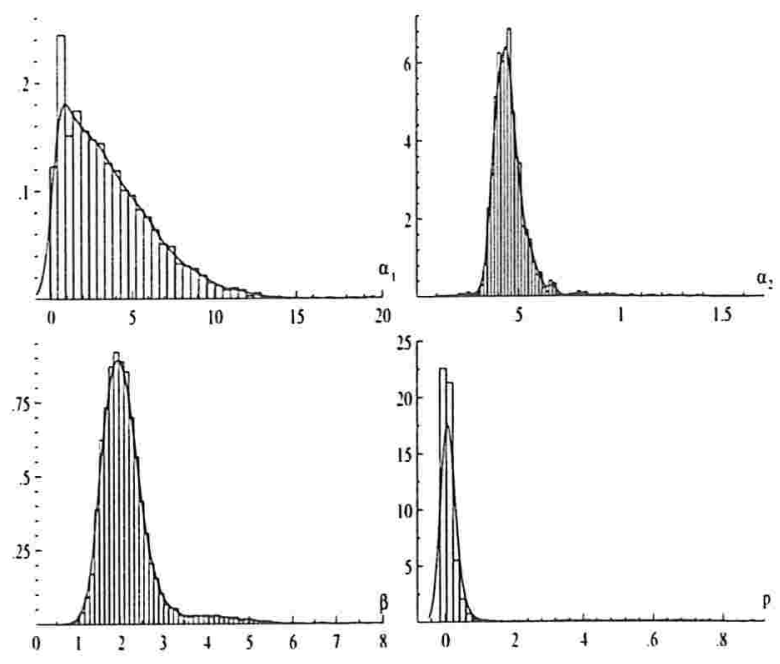

Figura 4.9: Densidades marginais a posteriori aproximados de $\alpha_{1}, \alpha_{2}, \beta$ e $p$ parâmetros do modelo de mistura de distribuições gamas

\section{Discriminação de modelos}

Como na subseção 5.1, na figura 4.10 temos o "plot" do log da razão CPO para os diferentes modelos com respeito aos outros contra o número de observações. Valores positivos do $\log$ da razão dos CPO indicam a preferência do primeiro modelo com respeito ao outro modelo. Na figura 4.10, modelo Weibull-exponenciada versus o modelo IDB o plot indica que 
41 das 50 observações sustentam ao modelo Weibull-exponenciada sobre o modelo IDB. Similarmente, 47 observações sustentam ao modelo Weibull-exponenciada sobre o modelo exponencial-potência, 40 observações sustentam ao modelo Weibull-exponenciada sobre o modelo de mistura de destribuições gamas, 28 observações sustentam o modelo IDB sobre o modelo exponencial-potência, 35 observações sustentam o modelo exponencial-potência sobre o modelo de mistura de gamas, 36 observações sustenta o modelo de mistura de distribuições gamas sobre o modelo IDB. Este criterio mostra que o modelo Weibullexponenciada é a que ajusta melhor aos dados da tabela 4.7.
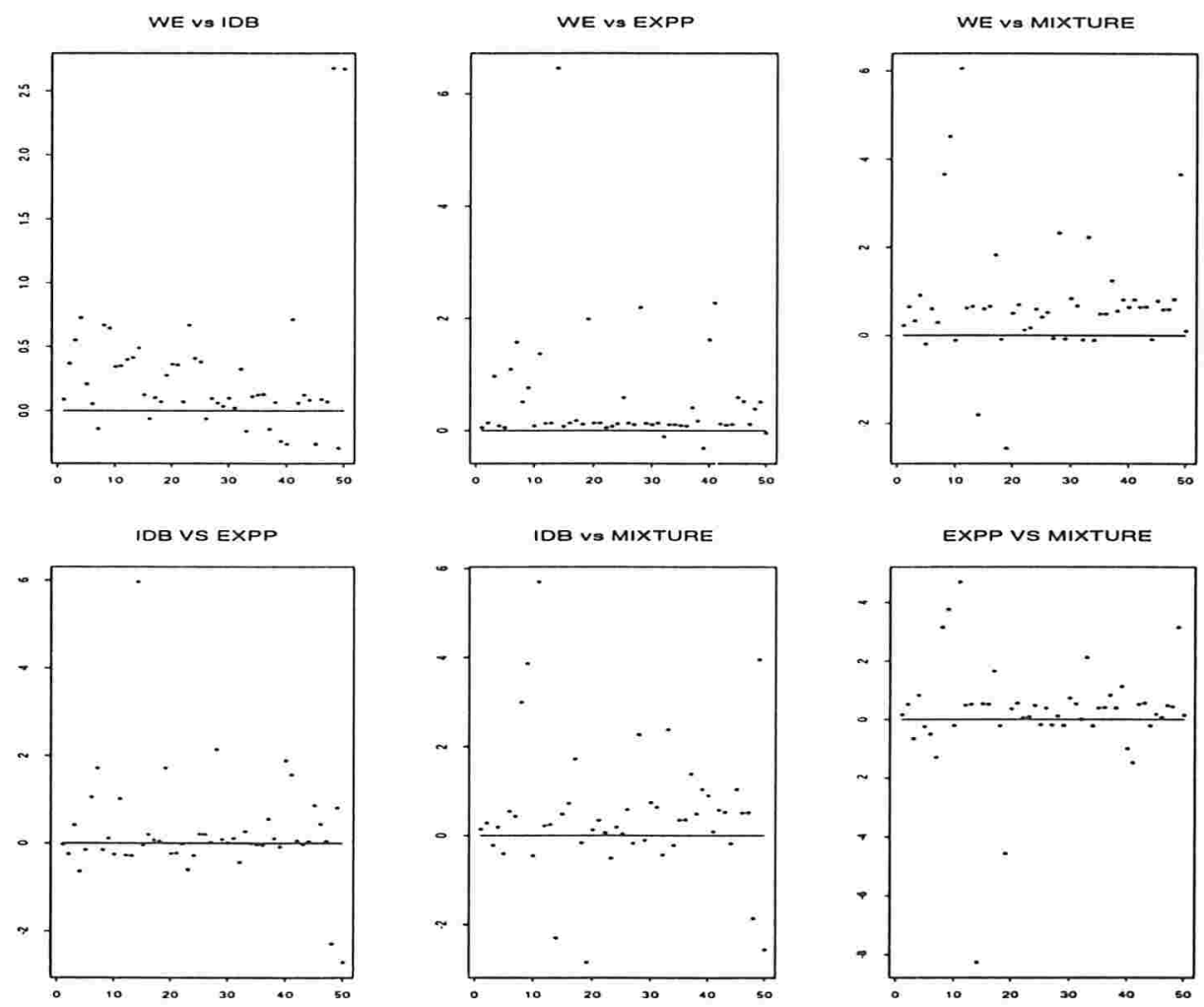

Figura 4.10: Gráfico do $\log$ da razão dos CPO para os diferentes modelos versus as observações

A mesma conclusões é também obtida pelo log do pseudo-fator de Bayes (1.11) (ver tabela 4.12). 
Tabela 4.12: $\log$ do pseudo-fator de Bayes

\begin{tabular}{|lc|}
\hline \hline Modelos & PSFB \\
\hline Weibull-exponenciada vs IDB & 13.9908 \\
Weibull-exponenciada vs exponencial-potência & 26.62271 \\
Weibull-exponenciada vs mistura de gamas & 38.0926 \\
IDB vs exponencial-potência & 12.63263 \\
IDB vs mistura de gamas & 24.10018 \\
exponencial-potência vs mistura de gamas & 11.46755 \\
\hline
\end{tabular}




\section{Capítulo 5}

\section{Comparação de dois modelos exponenciais com dados acelerados}

\subsection{Introdução}

Um teste acelerado consiste basicamente de uma variedade de métodos para reduzir a vida de produtos ou provocar a degradação da performance deste rapidamente. $\mathrm{O}$ principal objetivo é obter rapidamente os dados a serem analisados e desta forma produzir informações sobre a vida de um produto ou sua performance sob condições normais de uso. Um abrangente estudo deste ramo da confiabilidade assim como numerosas referências são dadas em Nelson (1990). Contudo, há poucos artigos relacionados a testes de hipóteses. Louzada-Neto, Bolfarine e Rodrigues (1991) desenvolveram uma análise Bayesiana para comparar duas populações sob condições aceleradas considerando a distribuição exponencial, para os tempos de sobrevivência, assumindo dados sob esquema de censura tipo II e densidade a priori não informativa de Jeffreys, para os parâmetros do modelo obteve a densidade a posteriori para o quociente dos tempos médios de vida das duas populações. Rodrigues, Bolfarine, e Louzada-Neto (1993) estenderam o trabalho de Louzada et al. (1991) para o caso de $p$ populações considerando para a comparação duas 
abordagens; a primeira baseada na estatística da razão de verossimilhança e a segunda baseada no fator de Bayes a posteriori (Aitkin, 1991). Nas duas abordagens eles consideraram a parametrização ortogonal (Cox e Reid, 1987) nos parâmentros do modelo. Na abordagen Bayesiana consideram a densidade a priori não informativa de Jeffreys para os parâmetros do modelo sob cada uma das hipóteses.

Neste Capítulo apresentamos uma análise Bayesiana alternativa para o problema de comparar duas populações exponenciais com dados acelerados propostos por Louzada et al. (1991), análise é baseada em métodos de simulação de Monte Carlo via Cadeias de Markov.

\subsection{Formulação do modelo}

Suponha que temos disponível 2 conjunto de dados acelerados independentes, sob o esquema de censura tipo II, tiradas de populações com densidade exponencial sob o estresse $V_{i}$ dado por

$$
f\left(t ; \theta_{i j}\right)=\frac{1}{\theta_{i j}} \exp \left\{-\frac{t}{\theta_{i j}}\right\}
$$

onde $\theta_{i j}$ (parâmetro desconhecido) $i=1, \ldots, k, \mathrm{j}=1,2$; é o tempo médio de falha da componente $j$ sob o estresse $V_{i}$.

Neste trabalho consideremos o modelo de lei de potência (veja, por exemplo, Nelson. 1990) como a relação entre o parâmetro de escala e o estresse $V_{i}$, isto é,

$$
\theta_{i j}=\frac{\alpha_{1 j}}{V_{i}^{\alpha_{2}}}
$$

onde $\alpha_{1 j}>0$ e $\alpha_{2 j} \in \mathcal{R}$ são parâmetros desconhecidos $j=1,2$.

De (5.2), a função de ligação que especifica a relação entre o tempo médio de falha e o nivel de estresse $V_{i}$ é dado por

$$
\log \left(\theta_{i j}\right)=X_{i}^{\prime} \beta_{j}
$$


onde $X_{i}^{\prime}=\left(1, \log \left(V_{i}^{-1}\right)\right)$ e o vetor de coeficientes de regressão é $\beta_{j}=\left(\beta_{1 j}, \beta_{2 j}\right)$ dado pela seguinte parametrização

$$
\beta_{1 j}=\log \left(\alpha_{1 j}\right), \quad \beta_{2 j}=\alpha_{2 j}
$$

O tempo médio de vida do $j$-ésimo componente sob o estresse $V_{i}$ pode ser escrito como

$$
\theta_{i j}=\exp \left\{X_{i}^{\prime} \beta_{j}\right\}
$$

Suponha que tem-se interesse em testar as seguintes hipóteses

$$
H_{0}: \theta_{11}=\theta_{12} \quad \text { vs } \quad H_{1}: \theta_{11} \neq \theta_{12} \text {. }
$$

Isto é, temos interesse em comparar os tempos médios de vida de duas populações no estresse usual $V_{1}$ que tem distribuição exponencial. Observe que sob $H_{0}$ em (5.6), $\theta_{11}=\theta_{12}$ implica $X_{1}^{\prime} \beta_{1}=X_{1}^{\prime} \beta_{2}(\operatorname{veja},(5.5))$.

Neste trabalho testamos as hipóteses usando o fator de Bayes. De (5.6) consideramos o modelo $M_{1}$ sob a $H_{0}$, ou seja, sob a suposição de que $\theta_{11}=\theta_{12}=\theta_{1}$ e o modelo $M_{2}$ sob $H_{1}$, ou seja, considerando-se $\theta_{11} \neq \theta_{12}$. Um tratamento Bayesiano para testar as hipóteses em (5.6) usando o o fator de Bayes a posteriori aproximado pelo método de Laplace é dado em Rodrigues et al. (1992).

\subsection{Teste de hipóteses Bayesiano}

Vamos descrever inicialmente alguns métodos alternativos para se obter aproximações através de métodos MCMC da verossimilhança preditiva sob o modelo $M_{j}, j=1,2$. definida em (1.5).

Conforme visto na seção 1.2.3 uma simple estimativa de Monte Carlo da função da verossimilhança preditiva, considerando amostra $\theta_{1}, \ldots, \theta_{n}$ da densidade a priori $\pi\left(\theta_{j} \mid M_{j}\right)$ 
é dada por

$$
\hat{f}_{1}\left(D \mid M_{j}\right)=\frac{1}{n} \sum_{i=1}^{n} L\left(\theta_{i} \mid M_{j}\right)
$$

Raftery (1995) argumenta que esse estimador não funciona bem se houver muita discrepância entre a densidade a priori e a verossimilhança. Uma estimativa alternativa para (1.5) é realizar amostragem por importância usando uma função densidade de importância $g(\theta)$, definindo

$$
\hat{f}_{2}\left(D \mid M_{j}\right)=\frac{\sum_{i=1}^{n} L\left(\theta_{i} \mid M_{j}\right) w_{i}}{\sum_{i=1}^{n} w_{i}},
$$

onde $\theta_{1}, \ldots, \theta_{n}$ é uma amostra da densidade de importância $g(\theta)$ e $w_{i}=\pi\left(\theta_{i}\right) / g\left(\theta_{i}\right)$.

Normalmente após um processo de simulação via cadeias de Markov, tem-se à disposição uma amostra $\theta_{1}, \ldots, \theta_{n}$ de $\pi\left(\theta \mid D, M_{j}\right)=k L\left(\theta \mid M_{j}\right) \pi\left(\theta \mid M_{j}\right)$. Logo, esses valores podem ser aproveitados através do estimador $\hat{f}_{2}$ bastando para isso tomar $g(\theta)=\pi\left(\theta \mid D, M_{j}\right)$. Nesse caso, o estimador se reduz a

$$
\hat{f}_{3}\left(D \mid M_{j}\right)=\left[\frac{1}{n} \sum_{i=1}^{n} L\left(\theta_{i} \mid M_{j}\right)^{-1}\right]^{-1}
$$

Este estimador é a média harmônica de valores da verossimilhança e foi originalmente proposto por Newton e Raftery (1994).

Uma outra aproximação para a verossimilhança preditiva (1.5) é obtida a partir do método de Laplace (veja por exemplo, Tierney e Kadane, 1986) a qual é dada por

$$
\hat{f}_{4}\left(D \mid M_{j}\right)=(2 \pi)^{d / 2}|\hat{\Sigma}|^{1 / 2} L\left(\hat{\theta} \mid M_{j}\right) \pi\left(\hat{\theta} \mid M_{j}\right)
$$

onde $d$ é a dimensão do vetor de parametros $\theta, \hat{\theta}$ é a moda da densidade a posteriori, isto é, o valor que maximiza $\ell(\theta) \propto \log \left(L\left(\theta \mid M_{j}\right) \pi\left(\theta \mid M_{j}\right)\right)$ e $\hat{\Sigma}$ é a inversa da matriz de segundas derivadas de $\ell(\hat{\theta})$. Raftery(1995) sugere para o cálculo desta aproximação utilizar a amostra $\theta_{1}, \ldots, \theta_{n}$ da densidade a posteriori. Uma estimativa da moda a posteriori é obtida calculando-se a posteriori para todos os pontos da amostra e escolhendo $\hat{\theta}$ de forma 
que $\pi(\hat{\theta})=\max _{i} \pi\left(\theta_{i} \mid D\right)$. Similarmente uma estimativa para a matriz de covariâncias a posteriori é dada pela matriz de covariâncias amostrais obtidas a partir da amostra gerada, ou seja,

$$
\hat{\Sigma}=\frac{1}{n} \sum_{i=1}^{n}\left(\theta_{i}-\bar{\theta}\right)\left(\theta_{i}-\bar{\theta}\right)^{\prime}
$$

onde $\bar{\theta}=\frac{1}{n} \sum_{i=1}^{n} \theta_{i}$. Raftery denomina esse estimador de Laplace-Metropolis e discute formas alternativas para o cálculo de estimativas de $\theta$ e $\Sigma$.

Neste Cápitulo, o fator de Bayes dado em (1.6) do modelo sob $H_{0}$ em (5.6) com respeito ao modelo sob $H_{1}$ em (5.6) denotada por $F B_{12}$, é computado com as estimativas das verossimilhanças preditivas propostas por Newton e Raftery (1994) dada em (5.8) e Raftery (1995) dada em (5.9). Rejeita-se $H_{0}$ se $F B_{12}<1$.

\subsection{A função de verossimilhança}

Nesta seção apresentamos a função de verossimilhança para os modelos $M_{1}$ e $M_{2}$ considerando dados sob o esquema de censura tipo II, isto é, o experimento termina quando $r_{i}$ falhas são observadas entre as $n_{i}$ unidades em testes no $i$-ésimo nível de estresse para $j=1,2$. Temos $t_{i j 1}, t_{i j 2}, \ldots, t_{i j r_{i}}$ observações não-censuradas e $n_{i}-r_{i}$ observações censuradas iguais a $t_{i j r_{i}}$, para $i=1, \ldots, k$.

A função de verossimilhança para $\beta_{j}^{\prime}=\left(\beta_{1 j}, \beta_{2 j}\right), j=1,2$. considerando $k$ níveis de estresse e um esquema de censura tipo II, é dada por

$$
L\left(\beta_{j}\right)=\prod_{i=1}^{k} e^{r_{i} X_{i}^{\prime} \beta_{j}} \exp \left\{-r_{i} \hat{\theta}_{i j} e^{-X_{i}^{\prime} \beta_{j}}\right\},
$$

onde $\hat{\theta}_{i j}=\frac{A_{i j}}{r_{i}}, A_{i j}=\sum_{l=1}^{k} t_{i j l}+\left(n_{i}-r_{i}\right) t_{i j r_{i}}$

A função de verossimilhaça para $\left(\beta_{1}, \beta_{2}\right)$ sob o modelo $M_{2}$ é dada por

$$
L\left(\beta_{1}, \beta_{2} \mid M_{2}, D\right)=\prod_{i=1}^{k} e^{-r_{i} X_{i}^{\prime}\left(\beta_{1}+\beta_{2}\right)} \exp \left\{-r_{i}\left(\hat{\theta}_{i 1} e^{-X_{i}^{\prime} \beta_{1}}+\hat{\theta}_{i 2} e^{-X_{i}^{\prime} \beta_{2}}\right)\right\}
$$


A função de verossimilhança para $\left(\beta_{1}, \beta_{2}\right)$ sob o modelo $M_{1}$ é dada por

$$
\begin{aligned}
L\left(\beta_{1}, \beta_{2} \mid M_{1}, D\right) & =\prod_{i=2}^{k} e^{-r_{i} X_{i}^{\prime}\left(\beta_{1}+\beta_{2}\right)} \exp \left\{-r_{i}\left(\hat{\theta}_{i 1} e^{-X_{i}^{\prime} \beta_{1}}+\hat{\theta}_{i 2} e^{-X_{i}^{\prime} \beta_{2}}\right)\right\} \\
& \times e^{-2 r_{1} X_{1}^{\prime} \beta_{1}} \exp \left\{-r_{1} e^{X_{1}^{\prime} \beta_{1}}\left(\hat{\theta}_{11}+\hat{\theta}_{12}\right)\right\}
\end{aligned}
$$

\subsection{Análise Bayesiana}

Um aspecto importante na análise Bayesiana é a especificação da densidade a priori $\pi\left(\beta_{j}\right)$ para $\beta_{j}, j=1, \ldots, k$. Com esse proposito, neste trabalho segue-se a abordagem de Bedrick et al.(1996) para formular uma densidade a priori para $\beta_{j}$ chamada "Data Augmentation priors" (DAP). O Procedimento DAP consiste em determinar uma priori para $\beta_{j}$ que tenha a forma funcional da função de verossimilhança. Também consideramos a obtenção da densidade a posteriori considerando densidades a priori não informativas e próprias, baseadas na distribuição normal e distribuição gama invertida.

\subsubsection{DAP para os modelos $M_{1}$ e $M_{2}$}

Para formular o DAP para os modelos $M_{1}$ e $M_{2}$, consideramos os níveis de estresse satisfazem $V_{1}<\ldots V_{k}$ e, seja $\tilde{X}_{i}=\left(1, \log \left(V_{i}\right)\right)^{\prime}, i=1,2$ o nível de estresse ou covariável seleccionado tal que a matriz $\tilde{X}$ de orden $2 \times 2$ seja não singular $\left(\tilde{X}_{1} \neq \tilde{X}_{2}\right.$. Daí temos que $\tilde{\theta}_{i j}=\exp \left\{\tilde{X}_{i} \beta_{j}\right\}$, implicando que

$$
\begin{gathered}
\tilde{\theta}_{j}=\exp \left\{\tilde{X} \beta_{j}\right\}, \quad j=1,2 \\
\text { onde } \tilde{\theta}_{j}^{\prime}=\left(\tilde{\theta}_{1 j}, \tilde{\theta}_{2 j}\right) \text { e } \tilde{X}=\left[\begin{array}{c}
\tilde{X}_{1} \\
\tilde{X}_{2}
\end{array}\right]_{2 \times 2} \text { é uma matriz não singular a qual implica } \\
\beta_{j}=\tilde{X}^{-1} \log \left(\tilde{\theta}_{j}\right), \quad j=1,2 .
\end{gathered}
$$




\section{Priori DAP para o modelo $M_{2}$}

De (5.12) e seguindo o procedimento de Bedrick et al.(1996) para o modelo $M_{2}$ temos que a densidade priori é dada por

$$
\pi\left(\beta_{1}, \beta_{2} \mid M_{2}\right) \propto \prod_{i=1}^{2} e^{-w_{i} \tilde{X}_{i}\left(\beta_{1}+\beta_{2}\right)} \exp \left\{-w_{i}\left(\hat{\tilde{\theta}}_{i 1} e^{-\tilde{X}_{i} \beta_{1}}+\hat{\tilde{\theta}}_{i 1} e^{-\tilde{X}_{i} \beta_{1}}\right)\right\} .
$$

A priori média condicional para $\left(\tilde{\theta}_{1}, \tilde{\theta}_{2}\right)$ (veja, Bedrick, et al.,1996) induzido por (5.13) via mudança de variáveis são $G I\left(w_{i}, w_{i} \hat{\tilde{\theta}}_{i j}\right)$ para, $i=1,2$ e $j=1,2$, independentes, com densidade conjunta

$$
\begin{aligned}
\pi\left(\tilde{\theta}_{1}, \tilde{\theta}_{2} \mid M_{2}\right) & \propto \tilde{\theta}_{11}^{-\left(w_{1}+1\right)} \exp \left\{-w_{1} \frac{\overline{\tilde{\theta}}_{11}}{\tilde{\theta}_{11}}\right\} \tilde{\theta}_{21}^{-w_{2}-1} \exp \left\{-w_{2} \frac{\hat{\tilde{\theta}}_{21}}{\tilde{\theta}_{21}}\right\} \\
& \times \tilde{\theta}_{12}^{-\left(w_{1}+1\right)} \exp \left\{-w_{1} \frac{\overline{\tilde{\theta}}_{12}}{\tilde{\theta}_{12}}\right\} \tilde{\theta}_{22}^{-w_{2}-1} \exp \left\{-w_{2} \frac{\hat{\tilde{\theta}}_{21}}{\tilde{\theta}_{21}}\right\} .
\end{aligned}
$$

O procedimento para atribuir valores a priori para $\left(w_{i}, \hat{\tilde{\theta}}_{i j}\right)$, é como segue: para uma escolha apropriada de $\tilde{A}_{i j}$ e nível de estresse $\tilde{X}_{i}$ fixado define-se para $i=1,2$, e $j=1,2$,

$$
\tilde{p}_{i}=\operatorname{Pr}\left[A_{i j}>\tilde{A}_{i j} \mid \tilde{X}_{i}, \tilde{\theta}_{i j}\right]=1-F\left(\frac{2 \tilde{A}_{i j}}{\tilde{\theta}_{i j}}\right),
$$

onde $F($.$) é a distribuição qui-quadrado com 2 r_{i}$ graus de liberdade. É razoável considerar $\hat{\tilde{\theta}}_{i j}$ como a informação a priori de $\tilde{\theta}_{i j}$ com o mesmo peso que $\hat{\theta}_{i j}$, ísto é, $w_{i}=r_{i}$. Para um valor de $\tau_{i}$, pode-se determinar $\gamma_{i}$ de modo que

$$
\operatorname{Pr}\left(\tilde{p}_{i}>\gamma_{i}\right)=\tau_{i}
$$

Daí tem-se

$$
\hat{\tilde{\theta}}_{i j}=\frac{\tilde{A}_{i j}}{r_{i}} U_{i j}
$$

onde

$$
U_{i j}=\frac{2 G^{-1}\left(\tau_{i}\right)}{F^{-1}\left(1-\gamma_{i}\right)}
$$

e $G($.$) é a distribuição \Gamma\left(w_{i}, 1\right)$, para $\mathrm{i}=1,2$. 
Para asegurar a independência entre os parâmetros $\tilde{\theta}_{1 j}$ e $\tilde{\theta}_{2 j}$ escolhe-se os níveis de estresse seguinte (para detalhes da justificativa desta escolha veja Bedrick et al., 1996 e Rodrigues, 1998):

$$
\begin{aligned}
& \tilde{X}_{1}=\left(1, \log \left(V_{1}\right)\right)^{\prime} \longrightarrow w_{1}=r_{1} \\
& \tilde{X}_{2}=\left(1, \log \left(V_{k}\right)\right)^{\prime} \longrightarrow \quad w_{2}=r_{k} .
\end{aligned}
$$

Usando o procedimento de mudança de variáveis estabelecidos em (5.4), obtem-se a seguinte distribuição a posteriori para o modelo $M_{2}$ :

$$
\begin{aligned}
\pi\left(\alpha_{11}, \alpha_{21}, \alpha_{12}, \alpha_{22} \mid M_{2}, D\right) \propto & \left(\alpha_{11} \alpha_{12}\right)^{-\left(r+r_{1}+r_{k}+1\right)}\left[\left(\prod_{1=1}^{k} V_{i}^{r_{i}}\right)\left(V_{1}^{r_{1}}\right)\left(V_{k}^{r_{k}}\right)\right]^{\alpha_{21}+\alpha_{22}} \\
& \exp \left\{-\sum_{j=1}^{2} \sum_{i=1}^{k} \frac{r_{i} \hat{\tau}_{i j} V_{i}^{\alpha_{2 j}}}{\alpha_{1 j}}\right\}
\end{aligned}
$$

onde

$$
\begin{aligned}
\hat{\tau}_{i j} & =\hat{\theta}_{i j}, & i=2, \ldots, k-1, & j=1,2 \\
& =\hat{\theta}_{1 j}+\hat{\tilde{\theta}}_{1 j}, & i=1, & j=1,2 \\
& =\hat{\theta}_{k j}+\hat{\tilde{\theta}}_{2 j}, & i=k, & j=1,2 .
\end{aligned}
$$

De (5.16) pode-se mostrar que as densidades condicionais completas de $\alpha_{11}, \alpha_{21}, \alpha_{12}$ e $\alpha_{22}$ são dadas por

(i) $\pi\left(\alpha_{11} \mid \alpha_{21}, \alpha_{12}, \alpha_{22}, M_{2}, D\right) \sim I G\left(r+r_{1}+r_{k} ; \sum_{i=1}^{k} r_{i} \hat{\tau}_{i 1} V_{i}^{\alpha_{21}}\right)$,

(ii) $\pi\left(\alpha_{21} \mid \alpha_{11}, \alpha_{12}, \alpha_{22}, M_{2}, D\right) \propto \exp \left\{-\frac{1}{\alpha_{11}} \sum_{i=1}^{k} r_{i} \hat{\tau}_{i 1} V_{i}^{\alpha_{21}}\right\}\left[\left(\prod_{1=1}^{k} V_{i}^{r_{i}}\right)\left(V_{1}^{r_{1}}\right)\left(V_{k}^{r_{k}}\right)\right]^{\alpha_{21}}$,

(iii) $\pi\left(\alpha_{12} \mid \alpha_{11}, \alpha_{21}, \alpha_{22}, M_{2}, D\right) \sim I G\left(r+r_{1}+r_{k} ; \sum_{i=1}^{k} r_{i} \hat{\tau}_{i 2} V_{i}^{\alpha_{22}}\right)$,

(iv) $\pi\left(\alpha_{22} \mid \alpha_{11}, \alpha_{21}, \alpha_{12}, M_{2}, D\right) \propto \exp \left\{-\frac{1}{\alpha_{12}} \sum_{i=1}^{k} r_{i} \hat{\tau}_{i 2} V_{i}^{\alpha_{22}}\right\}\left[\left(\prod_{1=1}^{k} V_{i}^{r_{i}}\right)\left(V_{1}^{r_{1}}\right)\left(V_{k}^{r_{k}}\right)\right]^{\alpha_{22}}$.

Observe que (i) e (iii) são distribuições padrões e (ii) e (iv) são distribuições não conhecidas devendo-se portanto utilizar o algoritmo de Metropolis-Hasting dentro do ciclo do amostrador de Gibbs para obter as distribuições marginais a posteriori aproximadas. 


\section{Priori DAP para $M_{1}$}

De (5.11) e seguindo o procedimento de Bedrick et al.(1996) para o modelo $M_{1}$ temos que a densidade a priori é dada por

$$
\begin{aligned}
\pi\left(\beta_{1}, \beta_{2} \mid M_{1}\right) & \propto e^{-2 w_{1} \tilde{X}_{1}^{\prime} \beta_{1}} \exp \left\{-2 w_{1} e^{-\tilde{X}_{1}^{\prime} \beta_{1}}\left(\frac{\hat{\tilde{\theta}}_{11}+\hat{\tilde{\theta}}_{12}}{2}\right)\right\} \\
& \times e^{-r_{2} \tilde{X}_{2}^{\prime}\left(\beta_{1}+\beta_{2}\right)} \exp \left\{-r_{2}\left(\hat{\tilde{\theta}}_{i 1} e^{-\tilde{X}_{2}^{\prime} \beta_{1}}+\hat{\tilde{\theta}}_{i 2} e^{-\tilde{X}_{2}^{\prime} \beta_{2}}\right)\right\},
\end{aligned}
$$

onde $\hat{\tilde{\theta}}_{i 1}$ é a informação a priori de $\tilde{\theta}_{i 1}$ e $w_{i}$ é o peso de $\hat{\tilde{\theta}}_{i 1}, i=1,2$.

A priori média condicional para $\left(\tilde{\theta}_{1}, \tilde{\theta}_{2}\right)$ (veja, Bedrick, et al.,1996) induzida por (5.17) via a técnica de mudança de variáveis é dada por

$$
\begin{aligned}
\pi\left(\tilde{\theta}_{1}, \tilde{\theta}_{2} \mid M_{1}\right) & \propto \tilde{\theta}_{11}^{-\left(2 w_{1}+1+1\right)} \exp \left\{-2 w_{1} \frac{\overline{\tilde{\theta}}_{1}}{\tilde{\theta}_{11}}\right\} \tilde{\theta}_{21}^{-w_{2}-1} \exp \left\{-w_{2} \frac{\hat{\tilde{\theta}}_{21}}{\tilde{\theta}_{21}}\right\} \\
& \times \tilde{\theta}_{22}^{-w_{2}-1} \exp \left\{-w_{2} \frac{\hat{\tilde{\theta}}_{22}}{\tilde{\theta}_{22}}\right\},
\end{aligned}
$$

onde $\overline{\tilde{\theta}}_{1}=\frac{\hat{\hat{\theta}}_{11}+\hat{\tilde{\theta}}_{12}}{2}$. De (5.18) observa-se que as densidades a priori são independentes, com

$$
\begin{aligned}
& \tilde{\theta}_{11} \sim \operatorname{IG}\left(2 w_{1}+1,2 w_{1} \tilde{\tilde{\theta}}_{1}\right), \\
& \tilde{\theta}_{21} \sim \operatorname{IG}\left(w_{2}, w_{2} \tilde{\theta}_{21}\right), \\
& \tilde{\theta}_{22} \sim \operatorname{IG}\left(w_{2}, w_{2} \tilde{\theta}_{22}\right),
\end{aligned}
$$

onde $I G(a, b)$ denota a densidade gama invertida com média $\frac{b}{a-1}$ e variância $\frac{b^{2}}{(a-1)^{2}(a-2)}$.

O procedimento para atribuir os valores a priori para $\overline{\tilde{\theta}}_{1}$, é como segue: para uma escolha apropriada de $\tilde{A}_{1}$ e nível de estresse $\tilde{X}_{1}$ fixado acima define-se,

$$
\tilde{p}_{1}=\operatorname{Pr}\left[A_{1}>\tilde{A}_{1} \mid \tilde{X}_{1}, \tilde{\theta}_{11}\right]=1-F\left(\frac{2 \tilde{A}_{1}}{\tilde{\theta}_{11}}\right),
$$

onde $F\left(\right.$.) é a distribuição qui-quadrado com $4 r_{1}$ graus de liberdade. Para um valor de $\tau_{1}$, pode-se determinar $\gamma_{1}$ de modo que

$$
\operatorname{Pr}\left(\tilde{p}_{1}>\gamma_{1}\right)=\tau_{1}
$$


Daí tem-se

$$
\overline{\tilde{\theta}}_{1}=\frac{\tilde{A}_{1}}{2 r_{1}} U_{11},
$$

onde

$$
U_{11}=\frac{2 G^{-1}\left(\tau_{1}\right)}{F^{-1}\left(1-\gamma_{1}\right)},
$$

e $G($.$) é a distribuição \Gamma\left(2 r_{1}+1,1\right)$, ou seja, a distribuição gama com parâmetros $2 r_{1}+1$ e 1 .

Segue-se o mesmo procedimento de $(5.22)$ para se atribuir valores a priori para $\hat{\tilde{\theta}}_{21}$ e $\hat{\tilde{\theta}}_{22}$, obtendo-se

$$
\hat{\tilde{\theta}}_{21}=\frac{\tilde{A}_{21}}{r_{k}} U_{21} \quad \text { e } \quad \hat{\tilde{\theta}}_{22}=\frac{\tilde{A}_{22}}{r_{k}} U_{22},
$$

sendo

$$
U_{21}=U_{22}=\frac{2 G^{-1}\left(\tau_{2}\right)}{F^{-1}\left(1-\gamma_{2}\right)},
$$

onde $F\left(\right.$.) é a distribuição de qui-quadrado com $2 r_{k}$ graus de liberdade e $G($.$) a distribuição$ $\Gamma\left(r_{k}, 1\right)$.

Usando a técnica de mudança de variaveis em (5.4), obtem-se a seguinte distribuição a posteriori para o modelo $M_{1}$ :

$$
\begin{aligned}
\pi\left(\alpha_{11}, \alpha_{21}, \alpha_{12}, \alpha_{22} \mid M_{1}, D\right) \propto & \alpha_{11}^{-\left(r+3 r_{1}+r_{k}+1\right)} \alpha_{12}^{-\left(r-r_{1}+r_{k}+1\right)}\left[\left(\prod_{1=1}^{k} V_{i}^{r_{i}}\right)\left(V_{1}^{3 r_{1}}\right)\left(V_{k}^{r_{k}}\right)\right]^{\alpha_{21}}(5.23) \\
& {\left[\left(\prod_{1=1}^{k} V_{i}^{r_{i}}\right)\left(V_{1}^{-r_{1}}\right)\left(V_{k}^{r_{k}}\right)\right]^{\alpha_{22}} \exp \left\{-\sum_{j=1}^{2} \sum_{i=1}^{k} \frac{r_{i} \hat{\eta}_{i j} V_{i}^{\alpha_{2 j}}}{\alpha_{1 j}}\right\}, }
\end{aligned}
$$

onde

$$
\begin{aligned}
\hat{\eta}_{i 1} & =\hat{\theta}_{i 1}, & & i=2, \ldots, k-1, \\
& =\hat{\theta}_{11}+\hat{\theta}_{12}+2 \overline{\tilde{\theta}}_{1}, & & i=1, \\
& =\hat{\theta}_{k 1}+\hat{\tilde{\theta}}_{21}, & & i=k
\end{aligned}
$$

$\mathrm{e}$,

$$
\hat{\eta}_{i 2}=\hat{\theta}_{i 2}, \quad i=2, \ldots, k-1,
$$




$$
\begin{array}{ll}
=0, & i=1, \\
=\hat{\theta}_{k 2}+\hat{\tilde{\theta}}_{22}, & i=k .
\end{array}
$$

De (5.23) pode-se mostrar que as densidades condicionais completas para $\alpha_{11}, \alpha_{21}$, $\alpha_{12}$ e $\alpha_{22}$ são dadas por

(i) $\pi\left(\alpha_{11} \mid \alpha_{21}, \alpha_{12}, \alpha_{22}, M_{1}, D\right) \sim I G\left(r+3 r_{1}+r_{k} ; \sum_{i=1}^{k} r_{i} \eta_{i 1} V_{i}^{\alpha_{21}}\right) ;$

(ii) $\pi\left(\alpha_{21} \mid \alpha_{11}, \alpha_{12}, \alpha_{22}, M_{1}, D\right) \propto \exp \left\{-\frac{1}{\alpha_{11}} \sum_{i=1}^{k} r_{i} \hat{\eta}_{i 1} V_{i}^{\alpha_{21}}\right\}\left[\left(\prod_{1=1}^{k} V_{i}^{r_{i}}\right)\left(V_{1}^{3 r_{1}}\right)\left(V_{k}^{r_{k}}\right)\right]^{\alpha_{21}}$;

(iii) $\pi\left(\alpha_{12} \mid \alpha_{11}, \alpha_{21}, \alpha_{22}, M_{1}, D\right) \sim I G\left(r-r_{1}+r_{k} ; \sum_{i=1}^{k} r_{i} \eta_{i 2} V_{i}^{\alpha_{22}}\right)$;

(iv) $\pi\left(\alpha_{22} \mid \alpha_{11}, \alpha_{21}, \alpha_{12}, M_{1}, D\right) \propto \exp \left\{-\frac{1}{\alpha_{12}} \sum_{i=1}^{k} r_{i} \hat{\eta}_{i 2} V_{i}^{\alpha_{22}}\right\}\left[\left(\prod_{1=1}^{k} V_{i}^{r_{i}}\right)\left(V_{1}^{-r_{1}}\right)\left(V_{k}^{r_{k}}\right)\right]^{\alpha_{22}}$.

onde $r=\sum_{i=1}^{k} r_{i}$. Observe que (i) e (iii) são distribuições conhecidas e (ii) e (iv) são distribuições intratáveis de ponto de vista de simulação devendo-se portanto, utilizar o algoritmo de Metropolis-Hasting dentro do ciclo do amostrador de Gibbs para se obter as distribuições marginais a posteriori aproximadas.

\subsubsection{Priori de Jeffreys}

Considerando o modelo (5.1) e a lei de potência (5.2), a função de verossimilhança de $\left(\alpha_{11}, \alpha_{21}, \alpha_{12}, \alpha_{22}\right)$ sob a hipótese $H_{0}$ (modelo $M_{1}$ ) em (5.6) é dada por

$$
\begin{aligned}
L\left(\alpha_{11} \alpha_{21}, \alpha_{12}, \alpha_{22} \mid M_{1}\right)= & \alpha_{11}^{-\left(r+r_{1}\right)} \alpha_{12}^{-\left(r-r_{1}\right)}\left[\left(\prod_{i=1}^{k} V_{i}^{r_{i}}\right) V_{1}^{r_{1}}\right]^{\alpha_{21}}\left[\left(\prod_{i=1}^{k} V_{i}\right) V^{-r_{1}}\right]^{\alpha_{22}} \\
& \times \exp \left\{-\sum_{j=1}^{2} \sum_{i=1}^{k} \frac{r_{i} \hat{\eta}_{i j}^{*} V_{i}^{\alpha_{2 j}}}{\alpha_{1 j}}\right\}
\end{aligned}
$$

onde

$$
\begin{aligned}
\hat{\eta}_{i 1}^{*} & =\hat{\theta}_{i 1}, \quad i=2, \ldots, k \\
& =\hat{\theta}_{11}+\hat{\theta}_{12}, \quad i=1 .
\end{aligned}
$$

$\mathrm{e}$

$$
\begin{aligned}
\hat{\eta}_{i 2}^{*} & =\hat{\theta}_{i 2}, \quad i=2, \ldots, k \\
& =0, \quad i=1,
\end{aligned}
$$


Pode-se mostrar que a matriz de informação de Fisher para o modelo $M_{1}$ é dada por

$$
I_{M_{1}}=\left(\begin{array}{cccc}
\frac{r_{+}+r_{1}}{\alpha_{11}^{2}} & -\frac{r_{1} \log \left(V_{1}\right)+\sum_{i=1}^{k} r_{i} \log \left(V_{i}\right)}{\alpha_{11}} & 0 & 0 \\
-\frac{r_{1} \log \left(V_{1}\right)+\sum_{i=1}^{k} r_{i} \log \left(V_{i}\right)}{\alpha_{11}} & r_{1} \log ^{2}\left(V_{1}\right)+\sum_{i=1}^{k} r_{i} \log ^{2}\left(V_{i}\right) & 0 & 0 \\
0 & 0 & \frac{\sum_{i=2}^{k} r_{i} \log \left(V_{i}\right)}{\alpha_{12}^{2}} & -\frac{\sum_{12}}{\alpha_{i=2}^{2} r_{i} \log \left(V_{i}\right)} \\
\alpha_{12} & \sum_{i=2}^{k} r_{i} \log ^{2}\left(V_{i}\right)
\end{array}\right)
$$

A função de verossimilhança de $\left(\alpha_{11}, \alpha_{21}, \alpha_{12}, \alpha_{22}\right)$ sob a $H_{1}$ (modelo $M_{2}$ ) em (5.6) é dada por

$$
L\left(\alpha_{11} \alpha_{21}, \alpha_{12}, \alpha_{22} \mid M_{2}\right)=\left(\alpha_{11} \alpha_{12}\right)^{-r}\left[\prod_{i=1}^{k} V_{i}^{r_{i}}\right]^{\alpha_{21}+\alpha_{22}} \exp \left\{-\sum_{j=1}^{2} \sum_{i=1}^{k} \frac{r_{i} \hat{\theta}_{i j} V_{i}^{\alpha_{2 j}}}{\alpha_{1} j}\right\} .
$$

Pode-se mostrar que a matriz de informação para o modelo $M_{2}$ é dada por

$$
I_{M_{2}}=\left(\begin{array}{cccc}
\sum_{\frac{r}{\alpha_{11}^{2}}}^{k} & -\frac{\sum_{i=1}^{k} r_{i} \log \left(V_{i}\right)}{\alpha_{11}} & 0 & 0 \\
-\frac{\sum_{i=1}^{k} r_{i} \log \left(V_{i}\right)}{\alpha_{11}} & \sum_{i=1}^{k} r_{i} \log ^{2}\left(V_{i}\right) & 0 & 0 \\
0 & 0 & \frac{r}{\sum_{12}^{2}} & -\frac{\sum_{i=1}^{k} r_{i} \log \left(V_{i}\right)}{\alpha_{12}} \\
0 & 0 & -\frac{\sum_{i=1}^{k} r_{i} \log \left(V_{i}\right)}{\alpha_{12}} & \sum_{i=2}^{k} r_{i} \log ^{2}\left(V_{i}\right)
\end{array}\right)
$$

De (5.25) e (5.27) pode-se mostrar que a priori não informativa de Jeffreys para os modelos $M_{1}$ e $M_{2}$ é dada por

$$
\pi\left(\alpha_{11}, \alpha_{21}, \alpha_{12}, \alpha_{22} \mid M_{j}\right) \propto \frac{1}{\alpha_{11} \alpha_{12}} .
$$


Considerando (5.24) e (5.28) e o teorema de Bayes pode-se mostrar que a distribuição a posteriori para o modelo $M_{1}$ é dada por

$$
\begin{aligned}
\pi\left(\alpha_{11}, \alpha_{21}, \alpha_{12}, \alpha_{22} \mid M_{1}, D\right) \propto & \alpha_{11}^{-\left(r+r_{1}+1\right)} \alpha_{12}^{-\left(r-r_{1}+1\right)}\left[\left(\prod_{i=1}^{k} V_{i}^{r_{i}}\right) V_{1}^{r_{1}}\right]^{\alpha_{21}} \\
& {\left[\left(\prod_{i=1}^{k} V_{i}^{r_{i}}\right) V_{1}^{-r_{1}}\right]^{\alpha_{22}} \exp \left\{-\sum_{j=1}^{2} \sum_{i=1}^{k} \frac{r_{i} \hat{\eta}_{i j}^{*} V_{i}^{\alpha_{2 j}}}{\alpha_{1} j}\right\} . }
\end{aligned}
$$

As distribuições condicionais para o algoritmo de Gibbs para esse modelo são dadas por

(i) $\pi\left(\alpha_{11} \mid \alpha_{21}, \alpha_{12}, \alpha_{22}, M_{1}, D\right) \sim I G\left(r+r_{1} ; \sum_{i=1}^{k} r_{i} \hat{\eta}_{i 1}^{*} V_{i}^{\alpha_{21}}\right)$,

(ii) $\pi\left(\alpha_{21} \mid \alpha_{11}, \alpha_{12}, \alpha_{22}, M_{1}, D\right) \propto \exp \left\{-\frac{1}{\alpha_{11}} \sum_{i=1}^{k} r_{i} \hat{\eta}_{i 1}^{*} V_{i}^{\alpha_{21}}\right\}\left[\left(\prod_{1=1}^{k} V_{i}^{r_{i}}\right)\left(V_{1}^{r_{1}}\right)\right]^{\alpha_{21}}$,

(iii) $\pi\left(\alpha_{12} \mid \alpha_{11}, \alpha_{21}, \alpha_{22}, M_{1}, D\right) \sim I G\left(r-r_{1} ; \sum_{i=1}^{k} r_{i} \hat{\eta}_{i 2}^{*} V_{i}^{\alpha_{22}}\right)$,

(iv) $\pi\left(\alpha_{22} \mid \alpha_{11}, \alpha_{21}, \alpha_{12}, M_{1}, D\right) \propto \exp \left\{-\frac{1}{\alpha_{12}} \sum_{i=1}^{k} r_{i} \hat{\eta}_{i 2}^{*} V_{i}^{\alpha_{22}}\right\}\left[\left(\prod_{1=1}^{k} V_{i}^{r_{i}}\right)\left(V_{1}^{-r_{1}}\right)\right]^{\alpha_{22}}$.

Utiliza-se o algoritmo de Metropolis-Hasting para obter amostras das densidades marginais em (ii) e (iv).

Considerando-se (5.26) e (5.28) e o teorema de Bayes pode-se mostrar que a distribuição a posteriori para o modelo $M_{2}$ é dada por

$$
\pi\left(\alpha_{11} \alpha_{21}, \alpha_{12}, \alpha_{22} \mid M_{2}\right) \propto\left(\alpha_{11} \alpha_{12}\right)^{-(r+1)}\left[\prod_{i=1}^{k} V_{i}^{r_{i}}\right]^{\alpha_{21}+\alpha_{22}} \exp \left\{-\sum_{j=1}^{2} \sum_{i=1}^{k} \frac{r_{i} \hat{\theta}_{i j} V_{i}^{\alpha_{2 j}}}{\alpha_{1} j}\right\} .
$$

As distribuições condicionais para o algoritmo de Gibbs para esse modelo são dadas por 
(i) $\pi\left(\alpha_{11} \mid \alpha_{21}, \alpha_{12}, \alpha_{22}, M_{2}, D\right) \sim I G\left(r ; \sum_{i=1}^{k} r_{i} \hat{\theta}_{i 1} V_{i}^{\alpha_{21}}\right)$

(ii) $\pi\left(\alpha_{21} \mid \alpha_{11}, \alpha_{12}, \alpha_{22}, M_{2}, D\right) \propto \exp \left\{-\frac{1}{\alpha_{11}} \sum_{i=1}^{k} r_{i} \hat{\theta}_{i 1} V_{i}^{\alpha_{21}}\right\}\left[\left(\prod_{1=1}^{k} V_{i}^{r_{i}}\right)\right]^{\alpha_{21}}$,

(iii) $\pi\left(\alpha_{12} \mid \alpha_{11}, \alpha_{21}, \alpha_{22}, M_{2}, D\right) \sim I G\left(r ; \sum_{i=1}^{k} r_{i} \hat{\theta}_{i 2} V_{i}^{\alpha_{22}}\right)$

(iv) $\pi\left(\alpha_{22} \mid \alpha_{11}, \alpha_{21}, \alpha_{12}, M_{2}, D\right) \propto \exp \left\{-\frac{1}{\alpha_{12}} \sum_{i=1}^{k} r_{i} \hat{\theta}_{i 2} V_{i}^{\alpha_{22}}\right\}\left[\left(\prod_{1=1}^{k} V_{i}^{r_{i}}\right)\right]^{\alpha_{22}}$.

Nas situações (ii) e (iv), utiliza-se o algoritmo de Metropolis-Hasting para obter amostras das densidades marginais.

\subsubsection{Prioris Próprias}

Neste caso consideremos as seguintes densidades a priori independentes para ambos os modelos

$$
\begin{aligned}
& \alpha_{1 j} \sim G I\left(a_{1 j}, b_{1 j}\right), \text { onde } a_{1 j} \text { e } b_{1 j}, j=1,2, \text { são conhecidos, } \\
& \alpha_{2 j} \sim N\left(\mu_{0 j}, \sigma_{0 j}^{2}\right), \text { onde } \mu_{0 j} \text { e } \sigma_{0} j, j=1,2 \text {, são conhecidos, }
\end{aligned}
$$

onde $N\left(\mu, \sigma^{2}\right)$ denota a distribuição normal com média $\mu$ e variância $\sigma^{2}$.

Considerando (5.24) e (5.31) e o teorema de Bayes pode-se mostrar que a distribuição a posteriori para o modelo $M_{1}$ é dada por

$$
\begin{aligned}
\pi\left(\alpha_{11} \alpha_{21}, \alpha_{12}, \alpha_{22} \mid M_{1}, D\right) \propto & \alpha_{11}^{-\left(r+r_{1}+a_{11}+1\right)} \alpha_{12}^{-\left(r-r_{1}+a_{12}+1\right)}\left[\left(\prod_{i=1}^{k} V_{i}^{r_{i}}\right) V^{r_{1}}\right]^{\alpha_{21}}\left[\left(\prod_{i=1}^{k} V_{i}\right) V_{1}^{-r_{1}}\right]^{\alpha_{22}} \\
& \exp \left\{-\sum_{j=1}^{2} \sum_{i=1}^{k} \frac{r_{i} \hat{\eta}_{i j}^{*} V_{i}^{\alpha_{2 j}}}{\alpha_{1} j}-\frac{1}{2} \sum_{j=1}\left(\frac{\alpha_{2 j}-\mu_{0 j}}{\sigma_{0 j}}\right)^{2}-\sum_{j=1}^{2} \frac{b_{i j}}{\alpha_{1 j}}\right\} .
\end{aligned}
$$

As distribuições condicionais para o algoritmo de Gibbs para esse modelo são dadas por 
$\begin{aligned} \text { (i) } \pi\left(\alpha_{11} \mid \alpha_{21}, \alpha_{12}, \alpha_{22}, M_{1}, D\right) & \sim I G\left(r+r_{1}+a_{11} ; b_{11}+\sum_{i=1}^{k} r_{i} \hat{\eta}_{i 1}^{*} V_{i}^{\alpha_{21}}\right), \\ \text { (ii) } \pi\left(\alpha_{21} \mid \alpha_{11}, \alpha_{12}, \alpha_{22}, M_{1}, D\right) & \propto \exp \left\{-\frac{1}{\alpha_{11}} \sum_{i=1}^{k} r_{i} \hat{\eta}_{i 1}^{*} V_{i}^{\alpha_{21}}-\frac{1}{2}\left(\frac{\alpha_{21}-\mu_{01}}{\sigma_{01}}\right)^{2}\right\} \\ & \times\left[\left(\prod_{1=1}^{k} V_{i}^{r_{i}}\right)\left(V_{1}^{r_{1}}\right)\right]^{\alpha_{21}}, \\ \text { (iii) } \pi\left(\alpha_{12} \mid \alpha_{11}, \alpha_{21}, \alpha_{22}, M_{1}, D\right) & \sim I G\left(r-r_{1}+a_{12} ; b_{12}+\sum_{i=1}^{k} r_{i} \hat{\eta}_{i 2}^{*} V_{i}^{\alpha_{22}}\right), \\ \text { (iv) } \pi\left(\alpha_{22} \mid \alpha_{11}, \alpha_{21}, \alpha_{12}, M_{1}, D\right) & \propto \exp \left\{-\frac{1}{\alpha_{12}} \sum_{i=1}^{k} r_{i} \hat{\eta}_{i 2}^{*} V_{i}^{\alpha_{22}}-\frac{1}{2}\left(\frac{\alpha_{22}-\mu_{02}}{\sigma_{02}}\right)^{2}\right\} \\ & \times\left[\left(\prod_{1=1}^{k} V_{i}^{r_{i}}\right)\left(V_{1}^{-r_{1}}\right)\right]^{\alpha_{22}} .\end{aligned}$

Em (i) e (iii) as distribuições são conhecida mas, em (ii) e (iv) as distribuições são de dificil tratamento e portanto, utiliza-se o algoritmo de Metropolis-Hasting para se obter amostras de suas densidades marginais.

Considerando (5.26) e (5.31) e o teorema de Bayes pode-se mostrar que a distribuição a posteriori para o modelo $M_{2}$ é dada por

$$
\begin{aligned}
\pi\left(\alpha_{11} \alpha_{21}, \alpha_{12}, \alpha_{22} \mid M_{2}\right) & \propto \alpha_{11}^{-\left(r+a_{11}+1\right)} \alpha_{12}^{-\left(r+a_{12}+1\right)}\left[\prod_{i=1}^{k} V_{i}^{r_{1}}\right]^{\alpha_{21}+\alpha_{22}} \\
& \times \exp \left\{-\sum_{j=1}^{2} \sum_{i=1}^{k} \frac{r_{i} \hat{\theta}_{i j} V_{i}^{\alpha_{2 j}}}{\alpha_{1} j}-\frac{1}{2} \sum_{j=1}^{2}\left(\frac{\alpha_{2 j}-\mu_{0 j}}{\sigma_{0 j}}\right)^{2}-\sum_{j=1}^{2} \frac{b_{i j}}{\alpha_{1 j}}\right\} .
\end{aligned}
$$

As distribuições condicionais para o algoritmo de Gibbs para esse modelo é dada por

(i) $\pi\left(\alpha_{11} \mid \alpha_{21}, \alpha_{12}, \alpha_{22}, M_{2}, D\right) \sim I G\left(r+a_{11} ; b_{11}+\sum_{i=1}^{k} r_{i} \hat{\theta}_{i 1} V_{i}^{\alpha_{21}}\right)$,

(ii) $\pi\left(\alpha_{21} \mid \alpha_{11}, \alpha_{12}, \alpha_{22}, M_{2}, D\right) \propto \exp \left\{-\frac{1}{\alpha_{11}} \sum_{i=1}^{k} r_{i} \hat{\theta}_{i 1} V_{i}^{\alpha_{21}}-\frac{1}{2}\left(\frac{\alpha_{21}-\mu_{01}}{\sigma_{01}}\right)^{2}\right\}\left[\left(\prod_{1=1}^{k} V_{i}^{r_{i}}\right)\right]^{\alpha_{21}}$,

(iii) $\pi\left(\alpha_{12} \mid \alpha_{11}, \alpha_{21}, \alpha_{22}, M_{2}, D\right) \sim I G\left(r+a_{12} ; b_{12}+\sum_{i=1}^{k} r_{i} \hat{\theta}_{i 2} V_{i}^{\alpha_{22}}\right)$,

(iv) $\pi\left(\alpha_{22} \mid \alpha_{11}, \alpha_{21}, \alpha_{12}, M_{2}, D\right) \propto \exp \left\{-\frac{1}{\alpha_{12}} \sum_{i=1}^{k} r_{i} \hat{\theta}_{i 2} V_{i}^{\alpha_{22}}-\frac{1}{2}\left(\frac{\alpha_{22}-\mu_{02}}{\sigma_{02}}\right)^{2}\right\}\left[\left(\prod_{1=1}^{k} V_{i}^{r_{i}}\right)\right]^{\alpha_{22}}$.

As distribuições de (i) e (iii) são conhecida mas, em (ii) e (iv) as distribuições não são conhecidas e portanto utiliza-se o algoritmo de Metropolis-Hasting para obter amostras de suas densidades marginais. 


\subsection{Aplicação}

Considere os dados da tabela 5.1. gerados assumindo-se uma distribuição exponencial e o modelo lei de potência com $\alpha_{1 j}=400$ e $\alpha_{2 j}=0.7, j=1,2$. Este dados são considerados em Rodrigues et al. (1993) e Louzada-Neto et al. (1991).

Tabela 5.1: Dados gerados $\operatorname{com} \alpha_{1 j}=400$ e $\alpha_{2 j}=0.7$

\begin{tabular}{|rrrrr|l|l|}
\hline$i$ & $V_{i}$ & $n_{i}$ & $r_{i}$ & $\theta_{i j}$ & Amostra 1 & Amostra 2 \\
\hline \hline 1 & 5 & 10 & 4 & 129.65 & $16.30,41,63$ & $14,27,42,58$ \\
2 & 10 & 10 & 6 & 78.81 & $15.21,36,39,54,59$ & $17,21,32,45,59,61$ \\
3 & 15 & 10 & 7 & 60.09 & $18.29,39,42,46,57,60$ & $14,23,26,35,42,78$ \\
4 & 20 & 10 & 8 & 49.13 & $19.20,36,37,41,45,45,57$ & $17,17,20,32,35,40,64,71$ \\
4 & 25 & 10 & 9 & 42.02 & $8.12,13,14,23,33,42,51,67$ & $10,13,21,28,33,44,53,69,75$ \\
\hline \hline
\end{tabular}

Considerando o modelo sob $H_{0}$ dado em (5.6) e a densidade a priori DAP para esse modelo com as seguintes informações a priori: $\tilde{A}_{1}=1017, \tilde{A}_{21}=330, \tilde{A}_{22}=425$, $\tau_{1}=0.9, \gamma_{1}=0,20, \tau_{2}=0,25 . \gamma_{2}=0,70, r_{1}=4$ e $r_{k}=9$, obtem-se $\overline{\tilde{\theta}}_{1}=161,44$, $\hat{\tilde{\theta}}_{21}=34,73$ e $\hat{\tilde{\theta}}_{22}=44,72$. Com essa escolha foram geradas 5 cadeias com 40.000 iterações cada uma e monitorou-se a convergência da amostra de Gibbs usando o método de Gelman e Rubin(1992), método este que utiliza a técnica de análise de variância para verficar se outras iterações são necessárias. Para cada parâmetro considerou-se as iterações $10.050^{\text {th }}, 10.100^{\text {th }}, \ldots, 20.000^{\text {th }}$ de cada cadeia, produzindo-se assim uma amostra final de tamanho 2.000. Na tabela 5.2, apresenta-se o resumo das distribuições a posteriori e, na figura 5.1 tem-se as densidades a posteriori aproximadas obtidas considerando-se as 2.000 amostras de Gibbs. Também, na tabela 5.2, tem-se o resultado da redução potêncial estimada de escala $\hat{R}$ (veja, Gelman e Rubin,1992) para todos os parâmetros. Neste caso o número de iterações consideradas foram suficientes para obter a convergência (ou seja, $\sqrt{\hat{R}}<1.1$ ), para todos os paramêtros, veja tabela 5.2 ). 
Tabela 5.2: Sumário a posteriori para o modelo sob $H_{0}$ com DAP

\begin{tabular}{|llllcc|}
\hline \hline & Média & Mediana & S.D & Intervalo de credibilidade $95 \%$ & $\hat{R}$ \\
\hline$\alpha_{11}$ & 655.49 & 554,11 & 614,56 & $(212,86 ; 1.644,64)$ & 1,008347 \\
$\alpha_{21}$ & 0,8326 & 0,8230 & 0,1986 & $(0,4607 ; 1,2252)$ & 1,003009 \\
$\alpha_{12}$ & $1.050,70$ & 366,30 & 2100,60 & $(21,60 ; 6.976,30)$ & 1,012548 \\
$\alpha_{22}$ & 0,6282 & 0,6271 & 0.5005 & $(-0,3277 ; 1,5976)$ & 1,0022077 \\
\hline \hline
\end{tabular}

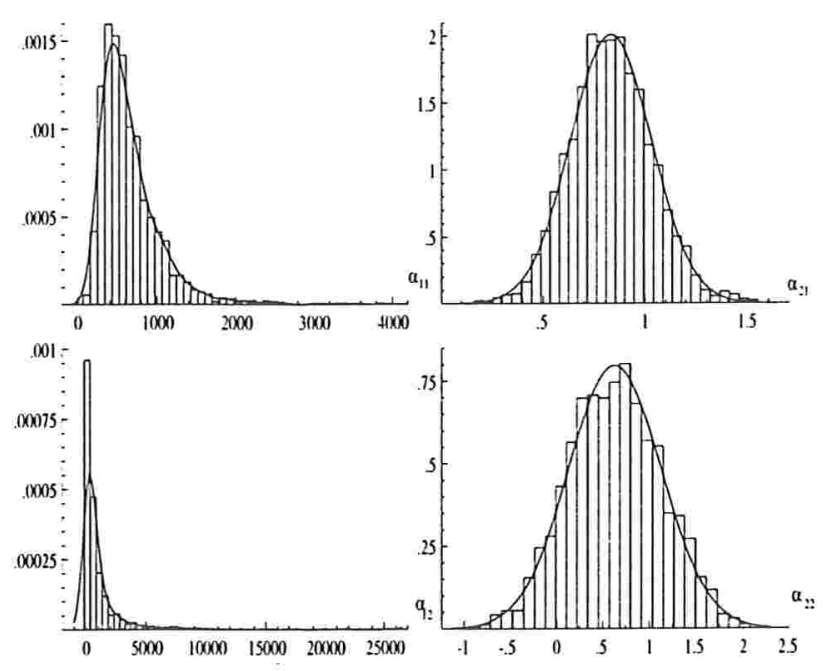

Figura 5.1: Densidades marginais a posteriori aproximados para $\alpha_{11}, \alpha_{21}, \alpha_{12}, \alpha_{22}$ do modelo sob $H_{0}$

Para o modelo sob $H_{1}$ dado em (5.6) e DAP com as seguintes informações a priori: $\tilde{A}_{11}=528, \tilde{A}_{21}=330, \tilde{A}_{12}=489, \tilde{A}_{22}=425, \tau_{1}=0,90, \gamma_{1}=0,20, \tau_{2}=0,25, \gamma_{2}=0,70$, $r_{1}=4$ e $r_{k}=9$, obtem-se $\hat{\tilde{\theta}}_{11}=159,901, \hat{\tilde{\theta}}_{21}=34,73, \hat{\tilde{\theta}}_{12}=148,09$ e $\hat{\tilde{\theta}}_{22}=44,72$. Com essa escolha foram geradas 5 cadeias com 40.000 iterações e monitorou-se a convergência da amostra Gibbs usando o método de Gelman e Rubin(1992). Para cada parâmetro considerou-se as iterações $20.050^{\text {th }}, 20.100^{\text {th }}, \ldots, 40.000^{\text {th }}$ de cada cadeia, que produz uma amostra final de tamanho 2.000. Na tabela 3, apresenta-se o resumo da distribuição a 
posteriori juntamente com o resultado da redução potêncial estimada de escala $\hat{R}$ (veja, Gelman e Rubin,1992) para todos os parâmetros, com valores bastante próximos de um. $\mathrm{Na}$ figura 5.2, tem-se as densidades marginais a posteriori para $\alpha_{11}, \alpha_{21}, \alpha_{12}$ e $\alpha_{22}$.

Tabela 5.3: Sumário a posteriori para o modelo sob $H_{1}$ com DAP

\begin{tabular}{|ccllcc|}
\hline \hline & Média & Mediana & S.D & Intervalo de credibilidade $95 \%$ & $\hat{R}$ \\
\hline$\alpha_{11}$ & 754,40 & 582,40 & 608,70 & $(167,84 ; 2.254,66)$ & 1,01388 \\
$\alpha_{21}$ & 0,8460 & 0,8392 & 0,2457 & $(0,3773 ; 1,3204)$ & 1,015013 \\
$\alpha_{12}$ & 546,90 & 421,10 & 503,10 & $(104,37 ; 1.862,40)$ & 1,012820 \\
$\alpha_{22}$ & 0,6721 & 0,6774 & 0.25947 & $(0,1741 ; 1,2099)$ & 1,001652 \\
\hline \hline
\end{tabular}
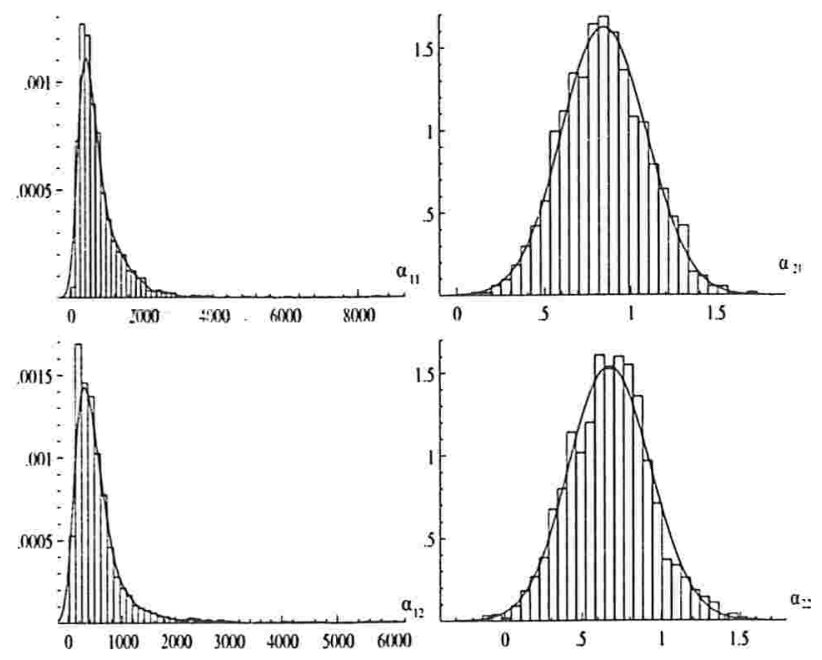

Figura 5.2: Densidades marginais a posteriori aproximados para $\alpha_{11}, \alpha_{21}, \alpha_{12}, \alpha_{22}$ do modelo sob $H_{1}$ com DAP

Considerando o modelo sob $H_{0}$ e densidade a priori não informativa de Jeffreys dada em (5.28), foram geradas 10 cadeias cada uma com 20.000 iterações. Foram consideradas as iterações $10.100^{\text {th }}, 10.200^{\text {th }}, \ldots, 20.000^{\text {th }}$ de cada cadeia, que produz uma amostra de tamanho 2.000. Na tabela 5.4, apresenta-se o resumo das distribuições a posteriori 
juntamente com o resultado da redução potêncial de escala para todos os parâmetros observamos que $\sqrt{\hat{R}}<1.1$, indicando a convergência e, na figura 5.3 , apresentamos as densidades marginais a posteriori aproximadas.

Tabela 5.4: Sumário a posteriori para o modelo sob $H_{0}$ com a densidade a priori não informativa.

\begin{tabular}{|llllcc|}
\hline \hline & Média & Mediana & S.D & Intervalo de credibilidade $95 \%$ & $\hat{R}$ \\
\hline$\alpha_{11}$ & 540,30 & 412,76 & 427,97 & $(95,01 ; 1.757,29)$ & 1.000945 \\
$\alpha_{21}$ & 0,7069 & 0,7177 & 0,2791 & $(0,1385 ; 1,2391)$ & 1.0001597 \\
$\alpha_{12}$ & $1.825,00$ & 524,00 & $4.952,00$ & $(28,60 ; 11.468,50)$ & 1.0611856 \\
$\alpha_{22}$ & 0.7440 & 0,7424 & 0,5364 & $(-0,2670 ; 1,8314)$ & 1.0902412 \\
\hline \hline
\end{tabular}
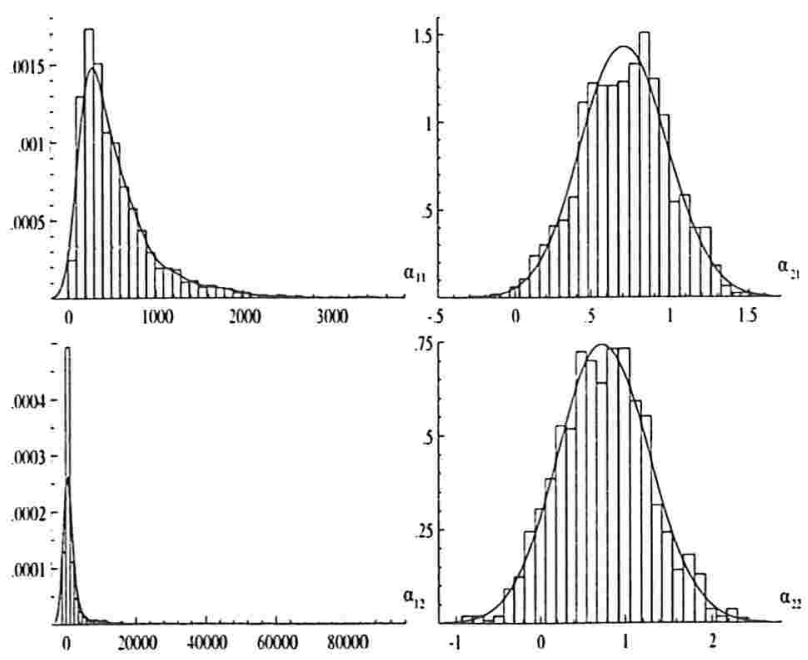

Figura 5.3: Densidades marginais a posteriori aproximados para $\alpha_{11}, \alpha_{21}, \alpha_{12}, \alpha_{22}$ do modelo sob $H_{0}$ com priori não informativa

Considerando o modelo sob $H_{1}$ com a densidade a priori não informativa de Jeffreys, foram simuladas 10 cadeias cada um com 20.000 iterações, considerado-se as iterações $10.050^{\text {th }}, 10.100^{\text {th }}, \ldots, 20.000^{\text {th }}$ de cada cadeia, produzindo uma amostra de tama- 
nho 2.000. Na tabela 5.5, apresenta-se o resumo a da distribuição a posteriori juntamente com o resultado da redução potêncial de escala, para todos os parâmetros observamos que $\sqrt{\hat{R}}<1.1$ indicando que convergência é atingida e, na figura 5.4, apresentamos as densidades marginais aproximadas a posteriori.

Tabela 5.5: Sumário a posteriori para o modelo sob $H_{1}$ com priori não informativa

\begin{tabular}{|llllcc|}
\hline \hline & Média & Mediana & S.D & Intervalo de credibilidade $95 \%$ & $\hat{R}$ \\
\hline$\alpha_{11}$ & 637,30 & 456,80 & 563,90 & $(92,46 ; 2.143,56)$ & 1.03744 \\
$\alpha_{21}$ & 0,7454 & 0,7405 & 0.2889 & $(0,15123 ; 1,3123)$ & 1.006111 \\
$\alpha_{12}$ & 520,20 & 309,70 & 581,30 & $(55,81 ; 2.403,59)$ & 1.05726 \\
$\alpha_{22}$ & 0,5848 & 0.5648 & 0.5828 & $(-0,0658 ; 1,2796)$ & 1.00526 \\
\hline \hline
\end{tabular}

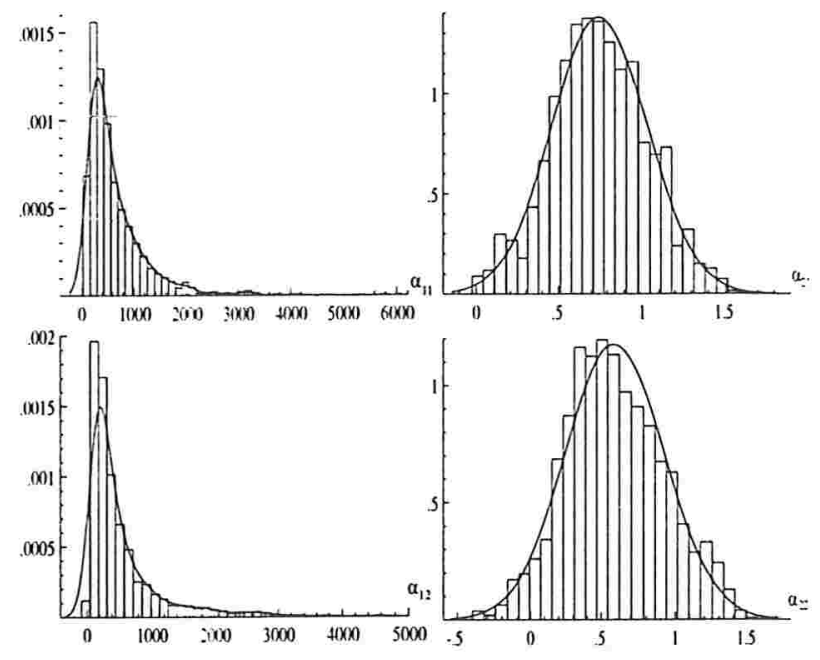

Figura 5.4: Densidades marginais a posteriori aproximados para $\alpha_{11}, \alpha_{21}, \alpha_{12}, \alpha_{22}$ do modelo sob $H_{1}$ com priori não informativa

Considerando o modelo sob $H_{0}$ e densidade a priori informativa (5.31) com, $a_{1 j}=3$, $b_{1 j}=10.000, \mu_{0 j}=0$ e $\sigma_{0 j}=100, j=1,2$, foram simulados 10 cadeias cada um com 20,000 iterações, considerando-se as iterações $10.100^{\text {th }}, 10.200^{\text {th }}, \ldots, 20.000^{\text {th }}$ de cada 
cadeia, que produz uma amostra de tamanho 1.000. Na tabela 5.6, apresenta-se o resumo das distribuições a posteriori juntamente com o resultado da redução potêncial de escala. onde para todos os parâmetros observamos que $\sqrt{\hat{R}}<1.1$ indicando convergência e, na figura 5.5, apresentamos as densidades marginais a posteriori aproximadas.

Tabela 5.6: Sumário a posteriori para o modelo sob $H_{0}$ com densidade a priori informativa

\begin{tabular}{|llllcc|}
\hline \hline & Média & Mediana & S.D & Intervalo de credibilidade $95 \%$ & $\hat{R}$ \\
\hline$\alpha_{11}$ & 337,28 & 325,76 & 88,18 & $(203,016 ; 502,754)$ & 1,000850 \\
$\alpha_{21}$ & 0,61848 & 0,61838 & 0,0850 & $(0,452688 ; 0,757692)$ & 1,0013037 \\
$\alpha_{12}$ & 403,55 & 338,64 & 235,14 & $(157,77 ; 848,33)$ & 1,001466 \\
$\alpha_{22}$ & 0,61733 & 0,60455 & 0,18073 & $(0,24091 ; 0,95937)$ & 1,0016882 \\
\hline \hline
\end{tabular}
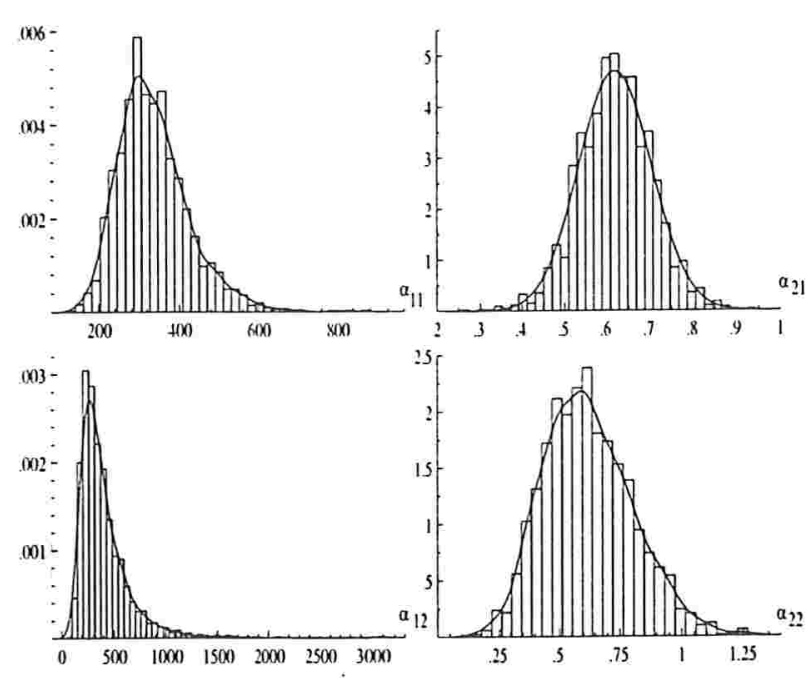

Figura 5.5: Densidades marginais a posterioris aproximados para $\alpha_{11}, \alpha_{21}, \alpha_{12}, \alpha_{22}$ do modelo sob $H_{0}$ com priori informativa

Considerando o modelo sob $H_{1}$ com a densidade a priori informativa (5.31) com $a_{1 j}=3, b_{1 j}=10.000, \mu_{0 j}=0$ e $\sigma_{0 j}=100, j=1,2$, forma gerados 10 cadeias cada um 
com 20.000 iterações, considerando-se as iterações $10.100^{\text {th }}, 10.200^{\text {th }}, \ldots, 20.000^{\text {th }}$ de cada cadeia, que produz uma amostra de tamanho 1.000. Na tabela 5.7, apresenta-se o resumo das distribuições a posteriori juntamente com o resultado da redução potêncial de escala, para todos os parâmetros e observamos que $\sqrt{\hat{R}}<1.1$, indicando a convergência e, na figura 5.6, apresentamos a densidades marginais a posteriori aproximadas.

Tabela 5.7: Sumário a posteriori para o modelo sob $H_{1}$ com densidade a priori informativa

\begin{tabular}{|llllcc|}
\hline \hline & Média & Mediana & S.D & Intervalo de credibilidade $95 \%$ & $\hat{R}$ \\
\hline$\alpha_{11}$ & 420,01 & 367,70 & 224,95 & $(209,15 ; 1.053,84)$ & 1.002560 \\
$\alpha_{21}$ & 0,6760 & 0,66530 & 0,17052 & $(0,4437 ; 1,0715)$ & 1.00532 \\
$\alpha_{12}$ & 362,$81 ;$ & 349,80 & 101,76 & $(233,86 ; 615,80)$ & 1.01023 \\
$\alpha_{22}$ & 0,6386 & 0,6402 & 0,0668 & $(0,4877 ; 0,7960)$ & 1.0070 \\
\hline \hline
\end{tabular}
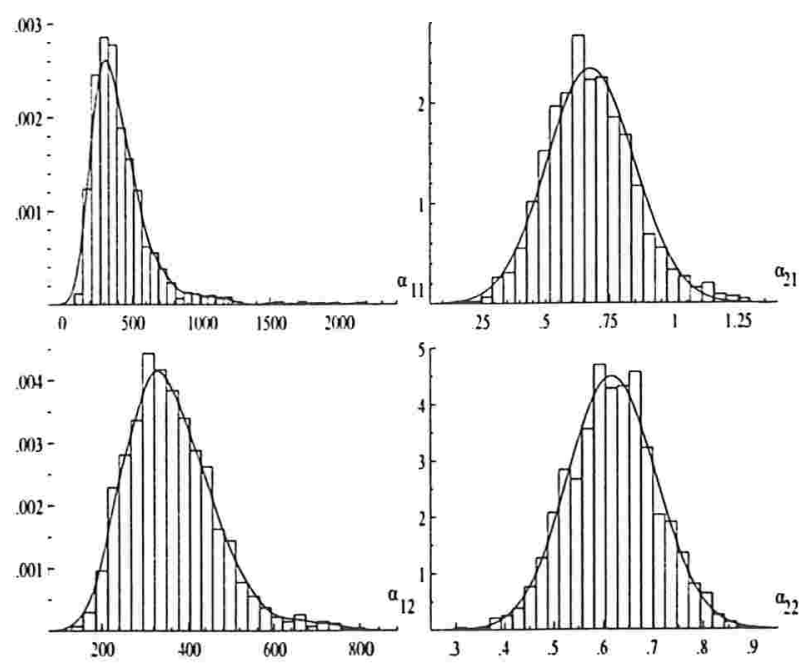

Figura 5.6: Densidades marginais a posteriori aproximados para $\alpha_{11}, \alpha_{21}, \alpha_{12}, \alpha_{22}$ do modelo sob $H_{1}$ com priori própria

Com as amostras de Gibbs geradas sob os modelos decorrentes das hipóteses $H_{0}$ e $H_{1}$, 
apresenta-se na tabela 5.8, o fator de Bayes com a estimativa da função de verossimilhança preditiva de Laplace-Metropolis, e a proposta por Newton e Raftery(1994). Daí, pode-se observar que o modelo sob $H_{0}$ é o que melhor se ajusta aos dados da tabela 5.1. O mesmo resultado foi obtido por Louzada-Neto et al. (1991).

Tabela 5.8: Fator de Bayes para descriminar o modelo sob $H_{0}$ vs o modelo sob $H_{1}$

\begin{tabular}{|llcc|}
\hline Fator de Bayes & Priori DAP & Priori não informativa & Informativa \\
\hline Laplace-Metropolis & 6,3316 & $1.802,98$ & 56,787 \\
Newton-Raftery & 308,8699 & 345,65 & 5,743 \\
\hline
\end{tabular}

Nas figuras $5.7,5.8$ e 5.9 apresenta-se as densidades marginais aproximados para $\theta_{11}$, $\theta_{12}$ e $\theta_{11}-\theta_{12}$ do modelo sob $H_{0}$ com DAP, priori não informativa e priori informativa, nas tabelas $5.9,5.10$ e 5.11 apresenta-se o resumo a posterior respectivo.
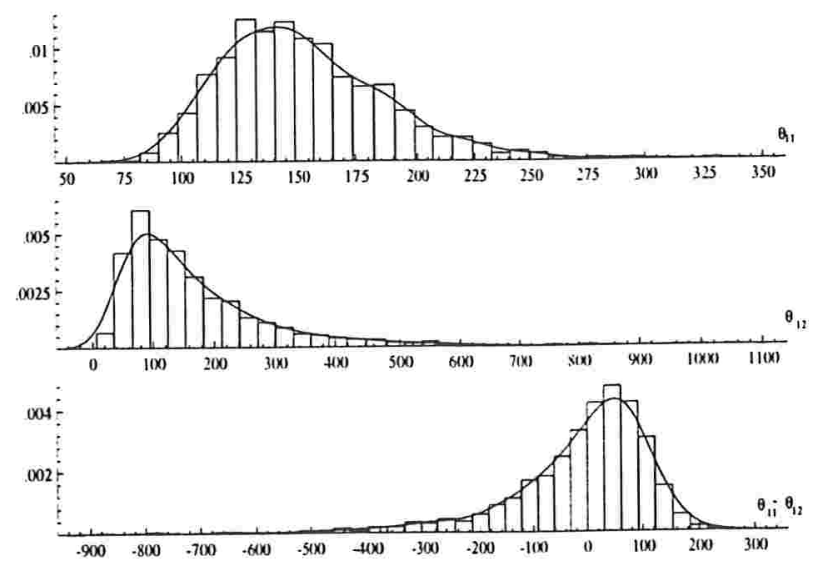

Figura 5.7: Densidades marginais a posterioris aproximados para $\theta_{11}, \theta_{12}$ e $\theta_{11}-\theta_{12}$ do modelo sob $H_{0}$ com DAP 
Tabela 5.9: Sumário a posteriori de (5.2) no nível de estresse usual, considerando DAP

\begin{tabular}{|llllc|}
\hline \hline & Média & Mediana & S.D & Intervalo de credibilidade 95\% \\
\hline$\theta_{11}$ & 152,96 & 147,99 & 35,12 & $(97,363 ; 238,449)$ \\
$\theta_{12}$ & 168,48 & 131,82 & 127,16 & $(37,75 ; 520,20)$ \\
$\theta_{11}-\theta_{12}$ & $-15,51$ & 15.01 & 132,36 & $(-367,57: 150.25)$ \\
\hline \hline
\end{tabular}
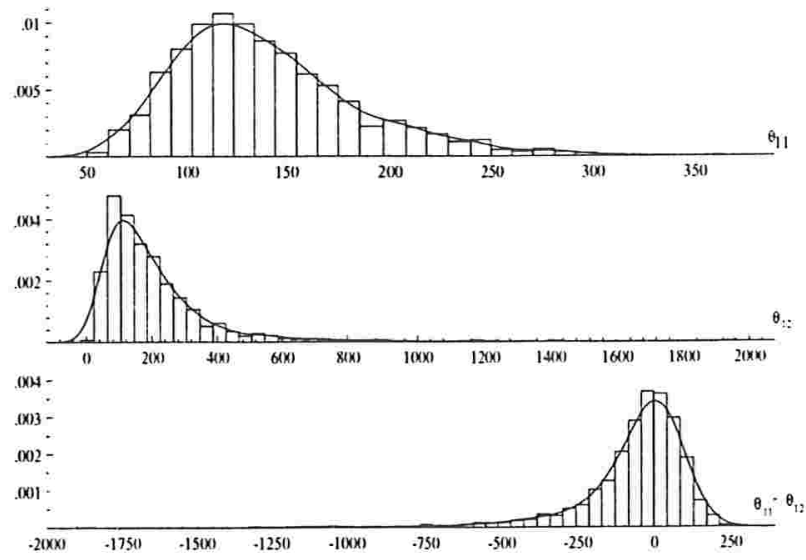

Figura 5.8: Densidades marginais a posteriori aproximados para $\theta_{11}, \theta_{12}$ e $\theta_{11}-\theta_{12}$ do modelo sob $H_{0}$ com densidade a priori não informativa de Jeffreys

Tabela 5.10: Sumário a posteriori de (5.2) com o nível de estresse usual considerando densidade a priori não informativa de Jeffreys

\begin{tabular}{|llllc|}
\hline \hline & Média & Mediana & S.D & Intervalo de credibilidade 95\% \\
\hline$\theta_{11}$ & 139,48 & 131,67 & 45,27 & $(71,60 ; 247,48)$ \\
$\theta_{12}$ & 205,02 & 156,70 & 136,80 & $(43,08 ; 6\rceil 4,48)$ \\
$\theta_{11}-\theta_{12}$ & $-65,55$ & 24,98 & 184,39 & $(-558,0 i: 155,04)$ \\
\hline \hline
\end{tabular}




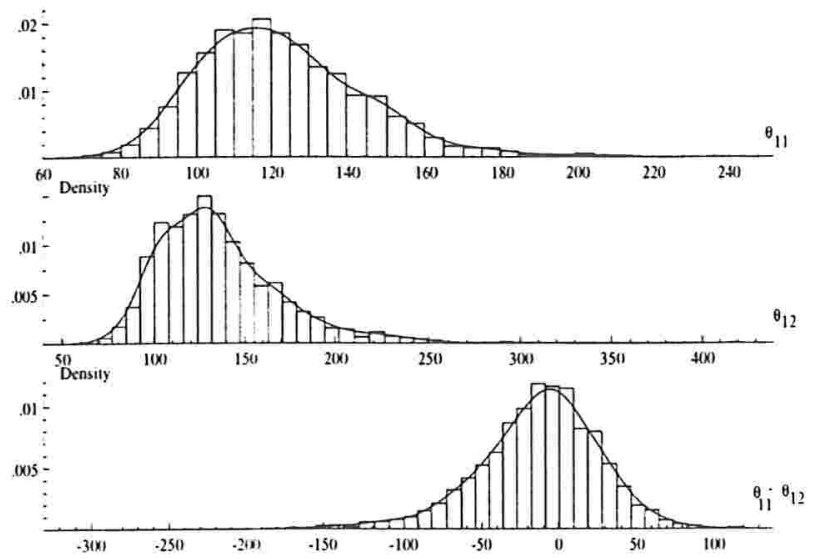

Figura 5.9: Densidades marginais a posteriori aproximados para $\theta_{11}, \theta_{12}$ e $\theta_{11}-\theta_{12}$ do modelo sob $H_{0}$ com densidade a priori informativa

Tabela 5.11: Sumário a posteriori de (5.2) com o nível de estresse usual considerando densidade a priori informativa

\begin{tabular}{|llllc|}
\hline \hline & Média & Mediana & S.D & Intervalo de credibilidade 95\% \\
\hline$\theta_{11}$ & 122,47 & 119,95 & 21,43 & $(87,728 ; 159,778)$ \\
$\theta_{12}$ & 135,58 & 130,15 & 33,79 & $(87,681 ; 198,605)$ \\
$\theta_{11}-\theta_{12}$ & $-13,104$ & $-9,763$ & 39,509 & $(-104,022 ; 44,671)$ \\
\hline \hline
\end{tabular}




\section{Capítulo 6}

\section{Modelo de mistura exponencial}

\subsection{Introdução}

Os modelos paramétricos comumente usados na análise de sobrevivência e na análise de confiabilidade assumem alguma família de distribuições, por exemplo Weibull, exponencial, gama, etc, para os tempos de vida. Assim, todos os indivíduos morrem (itens falham) se observamos por um período de tempo suficientemente longo. Essa afirmação parece não ser verdadeira em muitos contextos, já que, não considera a possibilidade de que indivíduos sobreviventes (ou itens que não falham) por longo período sejam observados . Isto induz a considerar modelos de mistura que permitam incluir sobreviventes ou indivíduos que não falham. A idéia aqui é que a distribuição dos tempos de falha somente seja considerada para os indivíduos que eventualmente morrem. Isto é, a distribuição é definida condicionalmente dados os indivíduos que morrem e é, irrelevante para os indivíduos sobreviventes ou indivíduos curados (itens que não falham).

A vantagem dos modelos de mistura com sobreviventes por longo período em relação, aos modelos de sobrevivência comums é que eles permitem um tipo de heterogeneidade limitada, mas, proveitosa, resultante do fato de que a subpopulação de sobreviventes por longo período tem taxa de falha zero. 
Modelos de mistura com sobreviventes por longo período foram adequadamente ajustados para dados de cancer por Boag (1949), Berkson e Gage (1952) e Pocock (1992) com aplicações a dados sobre a curabilidade de cancer do seio e Goldman (1984) para a curabilidade de cancer testicular. Varias generalizações dos modelos propostos por Boag (1949) e Berkson e Gage (1952) foram consideradas por Farewell (1977, 1982 e 1986) para vários estudos biomédicos com alguma discussão geral do uso dos modelos de mistura com sobreviventes por longo periodo de tempo em experimentos de sobrevivência; Greenhouse e Wolfe (1984) utilizam a teoria de risco competitivos. Aplicações para dados de AIDS tem sido considerados por Struthers e Farewell (1989).

Outras aplicações interessantes fora do contexto biomédico têm aparecido em pesquisas de mercado (Ascombe (1961)), em estudos de criminologia (Maltz e McCleary (1977), Maltz (1984), Schimidt e White (1988) e Broadhurst e Maller (1990)) e em estudos de desemprego (Dunsmuir, Tweedie, Flack e Mengersen (1989) e Yamaguchi (1992)). Na teoria de confiabilidade temos aplicações deste modelo em Meeker (1987). Neste Capítulo o objetivo é desenvolver uma análise Bayesiana para o problema através de métodos computacionais, fazendo comparações com técnicas de inferência clássica.

\subsection{Análise clássica do modelo de mistura exponen- cial}

Seja $b_{i}, 1 \leq i \leq n$, uma variável binária indicando que o $i$-ésimo indivíduo de uma população está em risco ou não com relação a algum tipo de falha $\left(b_{i}=1\right.$ se o indivíduo pertence ao grupo de risco e $b_{i}=0$ caso contrario). Considera-se que os $b_{i}$ são independentes e identicamente distribuidos tais que $P\left\{b_{i}=1\right\}=p \in(0,1)$ indica a proporção de indivíduos em risco e $P\left\{b_{i}=0\right\}=1-p$ a proporção de indivíduos imunes ou indivíduos curados ou itens que não falham.

As variáveis $b_{i}$ são não observadas mas, assume-se dado que os indivíduos pertencem 
ao grupo de risco, isto é, $b_{i}=1,1 \leq i \leq n$, os tempos de falha $t_{i}^{*}$ são independentes e identicamente distribuidos como distribuição exponencial com média comum $1 / \lambda, \lambda>0$, especificado pela densidade

$$
g(t ; \lambda \mid b=1)=\lambda e^{-\lambda t} . \quad t \geq 0 .
$$

Associado com o $i$-ésimo indivíduo está o tempo de censura $c_{i}, i=1, \ldots, n$. Consideramos que a distribuição dos tempos de censura são não informativos, isto é, ela não envolve $(\lambda, p)$, os parâmetros de interesse. Consideramos que $t_{i}^{*}$ e $c_{i}$ são estocasticamente independentes. Quando associado ao indivíduo $i . b_{i}=1$, observa-se tempo de censura ou falha $t_{i}=\min \left(t_{i}^{*}, c_{i}\right)$ e o indicador de censura $\delta_{i}=1$ se ocorre falha antes do final do estudo e $\delta_{i}=0$, caso contrário. Quando $b_{i}=0 . i=1, \ldots, n$ observa-se $t_{i}=c_{i}$ e $\delta_{i}=0$, neste caso todos os tempos são censurados.

\subsubsection{Estimadores de máxima verossimilhança}

Seja $\theta=(\lambda, p)^{\prime} \in\{\theta: \lambda>0,0<p \leq 1\}$, o vetor de parâmetros a ser estimado. Um indivíduo que morreu (iten que falha) no tempo $t_{i}$ contribui para função de verossimilhança com a parcela

$$
P\left\{b_{i}=1\right\} g\left(t_{i} ; \lambda \mid b_{i}=1\right)=p \lambda e^{-\lambda t_{i}}
$$

enquanto, que um indivíduo curado o iten que não falhou até o tempo $t_{i}$ contribui para a função de verossimilhança com a parcela

$$
P\left\{b_{i}=0\right\}+P\left\{b_{i}=1\right\} P\left(t_{i}^{*}>t_{i} ; \lambda \mid b_{i}=1\right)=1-p+p e^{-\lambda t_{i}},
$$

que é probabilidade do indivíduo ser um sobrevivente por longo período mais a probabilidade de um indivíduo sobreviver pelo menos até o tempo $t_{i}$. Portanto, a contribuição do $i$-ésimo indivíduo para a função de verossimilhança é dada por

$$
\left(p \lambda e^{-\lambda t_{i}}\right)^{\delta_{i}}\left(1-p+p \epsilon^{-\lambda t_{i}}\right)^{1-\delta_{i}}
$$


Logo, dada uma amostra $t_{1}, \ldots, t_{n}, \delta_{1}, \ldots, \delta_{n}$, a função de verosimilhança para $\theta$ é dada por

$$
L_{n}(\theta)=\prod_{i=1}^{n}\left(p \lambda e^{-\lambda t_{i}}\right)^{\delta_{i}}\left(1-p+p e^{-\lambda t_{i}}\right)^{1-\delta_{i}} .
$$

O estimador de máxima verossimilhança de $\theta$ é obtido maximizando-se (6.2), o que equivale a resolver o sistema de equações $S_{n}(\theta)=\sum_{i=1}^{n} s_{i}(\theta)=0, \operatorname{com} s_{i}(\theta)=$ $\left(s_{i 1}(\boldsymbol{\theta}), s_{i 2}(\boldsymbol{\theta})\right)^{\prime}$, onde

$$
\begin{aligned}
& s_{i 1}(\theta)=\frac{\partial \ell_{i}(\theta)}{\partial \lambda}=\frac{1}{\lambda}\left\{\delta_{i}\left(1-\lambda t_{i}\right)-\left(1-\delta_{i}\right) \frac{p \lambda t_{i} e^{-\lambda t_{i}}}{1-p+p e^{-\lambda t_{i}}}\right\}, \\
& s_{i 2}(\theta)=\frac{\partial \ell_{i}(\theta)}{\partial p}=\frac{1}{p}\left\{\delta_{i}-\left(1-\delta_{i}\right) \frac{p\left(1-e^{-\lambda t_{i}}\right)}{1-p+p e^{-\lambda t_{i}}}\right\},
\end{aligned}
$$

sendo $\ell_{i}=\log \left(L_{i}(\theta)\right)$ a $i$-ésima componente da função log-verossimilhança. Como não é possível obter de modo analítico os valores $\hat{\lambda}$ e $\hat{p}$ que satisfazem as equações acima devese, usar algum método iterativo, como por exemplo, o de Newton-Raphson. Esse método iterativo aparece como uma rotina do pacote Ox (veja Doornik 1998).

\subsubsection{Inferência sobre os parâmetros $\lambda$ e $p$}

A inferência sobre os parâmetros são feitas usualmente através da normalidade assintótica dos estimadores de máxima verossimilhança. Assim, para $\hat{\lambda}$ e $\hat{p}$, EMVs de $\lambda$ e $p$, ponto interior do espaço paramétrico e sob certas condições de regularidade (veja por exemplo, Ghitany e Maller, 1992) tem-se o seguinte resultado, para $n \longrightarrow \infty$ :

$$
I_{n}^{1 / 2}(\hat{\lambda}, \hat{p})\left[\left(\begin{array}{l}
\hat{\lambda} \\
\hat{p}
\end{array}\right)-\left(\begin{array}{l}
\lambda \\
p
\end{array}\right)\right] \stackrel{D}{\longrightarrow} N\left(0, I_{2}\right),
$$

onde $I_{n}^{1 / 2}(\lambda, p)$ é a matriz raiz quadrada da matriz de informação de Fisher e $N\left(0, I_{2}\right)$ denota a distribuição normal bivariada padrão ( $I_{2}$ é a matriz identidade de dimensão 
$2 \times 2$ ). Ghitany e Maller (1992) considerando os tempos de censura como sendo variáveis aleatórias independentes e identicamente distribuídas não degeneradas no ponto zero mostraram que os elementos da matriz de informação de Fisher são dados por:

$$
\begin{aligned}
& I_{n}^{11}=\frac{n p}{\lambda^{2}} E\left[1-e^{-\lambda c}-\frac{(1-p)(\lambda c)^{2} e^{-\lambda c}}{1-p\left(1-e^{-\lambda c}\right)}\right] \\
& I_{n}^{22}=\frac{n}{p} E\left[\frac{1-e^{-\lambda c}}{1-p\left(1-e^{-\lambda c}\right)}\right] \\
& I_{n}^{12}=I_{n}^{21}=\frac{n}{\lambda} E\left[\frac{\lambda c e^{-\lambda c}}{1-p\left(1-e^{-\lambda c}\right)}\right] .
\end{aligned}
$$

Observe em (6.6), que se as variáveis aleatórias dos tempo de censura convergem quase certamente a $\infty$, os elementos da matriz de informação de Fisher se reduzem a

$$
I_{n}^{11}=\frac{n p}{\lambda^{2}}, \quad I_{n}^{22}=\frac{n}{p(1-p)} . \text { e } I_{n}^{12}=I_{n}^{21}=0
$$

de modo que as variâncias assintóticas de EMV de $\lambda$ e $p$ são dadas por $\lambda^{2} / n p$ e $p(1-p) / n$, respectivamente.

Suponha que temos interesse em provar que a proporção de indivíduos imunes ou a proporção de indivíduos curados de certa enfermidade é nula, contra a alternativa que não é, isto é,

$$
\begin{aligned}
& H_{0}: \quad p=1 . \\
& H_{1}: p<1 .
\end{aligned}
$$

A estatística da razão de verossimilhança para testar $H_{0}$ é dada por

$$
R V_{n}=2\left(\ell_{n}(\hat{\lambda}, \hat{p})-\ell_{n}(\tilde{\lambda}, 1)\right)
$$

onde $\hat{\lambda}$ e $\hat{p}$ são EMV irrestritos e $\tilde{\lambda}$ é o EMV sob $H_{0}$ dada em (6.8). Sob certas condições de regularidade, Ghitany e Maller (1995) mostraram que a distribuição assintótica de (6.9) é uma mistura 50\%-50\% da distribuição qui-quadrado com um grau de liberdade e um ponto de massa em 0 , isto é, 


$$
P\left(R V_{n} \leq x\right)=\frac{1}{2}+\frac{1}{2} P\left(\chi_{(1)}^{2} \leq x\right)
$$

\subsubsection{Um estudo de simulação}

Nesta subseção apresenta-se um estudo de simulação para investigar o comportamento da distribuição assintótica da estatística do teste (6.10) para pequenas amostras. O estudo de simulação é baseado num modelo exponencial com média um para os tempo de vida e a variável $c_{i}$ (independente dos tempo de vida $t_{i}^{*}$ ) correspondente ao mecanismo de censura, foi gerada como uma variável aleatória uniforme no intervalo $(0,5)$. Várias combinações de tamanhos amostrais $(n=20,30,50,100)$ foram consideradas no estudo. Mil réplicas foram geradas para cada simulação. Na tabela 6.1, apresenta-se os os níveis de significância empíricos para os diferentes tamanhos amostrais. Observa-se que os níveis de significância empíricos são quase o dobro dos níveis nominais teóricos o que indica que o teste não é preciso para pequenas amostras.

Tabela 6.1: Percentagem de rejeição de $H_{0}: p=1$ no modelo de sobrevivência com sobreviventes por longo período em 1000 replicações para diferentes tamanhos amostrais

\begin{tabular}{|llll|}
\hline \hline$n$ & $10 \%$ & $5 \%$ & $1 \%$ \\
\hline 20 & 22.5 & 11.2 & 1.7 \\
30 & 22.3 & 10.2 & 1.7 \\
50 & 20.6 & 8.5 & 1.3 \\
100 & 18.2 & 8.0 & 1.2 \\
\hline
\end{tabular}




\subsubsection{Comparação de proporções de indivíduos imunes entre grupos}

Suponha que existem $n$ indivíduos em um estudo, foram aplicados $G$ tratamentos (grupos) diferentes, com $n_{g}$ indivíduos para o tratamento $g, g=1, \ldots, G$ e, queremos verificar se existem diferenças nas proporções de indivíduos não imunes nos grupos. Isto é, queremos provar as hipóteses

$$
\begin{aligned}
& H_{0}: 0<p_{1}=\ldots=p_{g}=p<1, \\
& H_{1}: p_{i} \neq p, \quad \text { para algum, } i=1, \ldots, G,
\end{aligned}
$$

onde $0<p<1$.

Githany. Maller e Zhou (1995) sob certas condições de regularidade mostraram que a estatística da razão de verossimilhança para testar (6.11),

$$
R V_{n}=-2\left[\ell_{n}(\hat{\theta})-\ell_{n}(\tilde{\theta})\right],
$$

tem, quando $n \longrightarrow \infty$, distribuição qui-quadrado com $G-1$ graus de liberdade, onde $\tilde{\theta}$ é o EMV irrestrito e $\hat{\theta}$ é o EMV sob $H_{0}$.

Githany et al. (1995) sugerem testar (6.11) em dois estágios, onde num primeiro estágio testa-se se a proporção de imunes nos grupos é nula, ísto é,

$$
\begin{aligned}
& H_{0}: p_{1}=\ldots=p_{G}=1, \\
& H_{1}: \quad p_{i} \neq 1, \quad \text { para algum, } i=1, \ldots, G .
\end{aligned}
$$

A estatística de teste para hipótese acima é dada por

$$
d_{n}=2 \sum_{g=1}^{G} \hat{\ell}_{n}(g) \stackrel{D}{\longrightarrow} X_{1}+\ldots+X_{G}
$$

onde $\hat{\ell}_{n}(g)=\ell_{n}\left(\hat{\lambda}_{(g)}, \hat{p}_{(g)}\right)-\ell_{n}\left(\hat{\lambda}_{(g)}, 1\right)$ e $X_{g}$ tem a distribuição dada em (6.10). Se aceitamos $H_{0}$ em (6.13) concluimos que não há imunes em nenhum grupo e a análise procede 
como uma análise de sobrivivência ordinária. Se rejeita-se $H_{0}$ então consideramos a hipótese (6.11) como um segundo estágio. A distribuição assintótica da estatística em (6.13) é complicada e uma tabela é apresentada em Maller e Zhou (1996). O comportamento da estatística para $n$ pequeno deixa bastante a desejar como vemos a seguir. Um problema clássico em aberto é testar alternativas restritas com relação aos $p_{i}$ 's, como por exemplo, $H_{1}: p_{1} \leq \ldots \leq p_{G}$ com pelo menos uma desigualdade estrita. Propomos uma solução parcial usando o enfoque bayesiano.

\subsubsection{Estudo de simulação}

Nesta subseção apresentamos um estudo de simulação de Monte Carlo com a finalidade de comparar os níveis de significância empíricos e teóricos para testar $H_{0}$ em (6.13). Neste estudo consideramos dois grupos $(G=2)$ com diferentes tamanhos amostrais $\left(n_{1}=n_{2}=20,30,50,100\right)$. As amostras foram geradas a partir de $H_{0}(6.13)$; para os tempos de vida, consideramos um modelo exponencial com média um e para os tempos de censura independentemente dos tempos de vida consideramos a variável aletória uniforme no intervalo $(0,5)$ para os dois grupos. Na tabela 6.2 , apresenta-se os níveis de significância empíricos, onde observa-se que os níveis de significância são praticamente o dobro dos teóricos.

Tabela 6.2: Percentagem de rejeição de $H_{0}: p_{1}=p_{2}=1$ no modelo de sobrevivência por longo periodo com 1000 replicações

\begin{tabular}{|llll|}
\hline \hline$n_{1}=n_{2}$ & $10 \%$ & $5 \%$ & $1 \%$ \\
\hline 20 & 23.9 & 12.2 & 2.5 \\
30 & 23.1 & 11.8 & 2.1 \\
50 & 22.3 & 10.7 & 2.1 \\
100 & 20.1 & 10.3 & 2.1 \\
\hline
\end{tabular}




\subsection{Análise Bayesiana para modelos de mistura ex- ponencial}

Como foi discutido na seção anterior a distribuição dos EMVs somente são conhecidas assintóticamente, tornando-se assim um problema sério, uma vez que, em geral, trabalhase com amostras não muito grandes. Desta forma, inferências (intervalos de confiança, teste de hipóteses, etc.) baseadas nessas estatísticas podem não ser confiáveis, conforme mostram os estudos de simulação desenvolvidos anteriormente.

Um procedimento alternativo que pode contornar esse problema é através da utilização de métodos Bayesianos. Conforme vistos nos capítulos anteriores, o uso de métodos Bayesianos, além de ser uma alternativa de análise, permite a incorporação de conhecimento a priori através de uma densidade a priori que seja informativa. Caso não exista conhecimento a priori, ou seja difícil expressá-lo, considera-se densidades a priori não informativas.

\subsubsection{Análise Bayesiana do modelo de mistura considerando a densidade a priori não informativa de Jeffreys}

Considerando (6.7), pode-se mostrar que a densidade a priori não informativa de Jeffreys para os parâmetros $\lambda$ e $p$ é dada por,

$$
\pi(\lambda, p) \propto \lambda^{-1}(1-p)^{-1 / 2}
$$

Considerando (6.15) e (6.2) e o teorema de Bayes pode-se mostrar que a densidade a posteriori conjunta para $\lambda$ e $p$ do modelo de mistura exponencial é dada por

$$
\pi(\lambda, p \mid D) \propto p^{r}(1-p)^{-1 / 2} \lambda^{r-1} e^{-R \lambda} \prod_{i=1}^{n}\left(1-p+p e^{-\lambda t_{i}}\right)^{1-\delta_{i}}
$$

onde $D$ é o conjunto de dados observados, $r=\sum_{i=1}^{n} \delta_{i}$ e $R=\sum_{i=1}^{n} \delta_{i} t_{i}$. 
O teorema 6.3.1 garante que a densidade conjunta a posteriori em (6.16) é própria.

Teorema 6.3.1 Sob o modelo mistura exponencial, se a densidade a priori é dada por (6.15) então a densidade a posteriori (6.16) é própria.

Prova: Desde que $\left(1-p+p e^{-\lambda t_{i}}\right) \leq 1$ para todo $p$ e $i=1, \ldots, n$, temos que

$$
\begin{aligned}
\int_{0}^{1} \int_{0}^{\infty} \pi(\lambda, p \mid D) d \lambda d p & \propto \int_{0}^{1} \int_{0}^{\infty} p^{r}(1-p)^{-1 / 2} \lambda^{r-1} e^{-R \lambda} \prod_{i=1}^{n}\left(1-p+p e^{-\lambda t_{i}}\right)^{1-\delta_{i}} d \lambda d p \\
& \leq \int_{0}^{1} \int_{0}^{\infty} p^{r}(1-p)^{-1 / 2} \lambda^{r-1} e^{-R \lambda} d \lambda d p \\
& =\frac{\Gamma(r+1) \Gamma(1 / 2) \Gamma(r)}{\Gamma(r+3 / 2) R^{r}}<\infty
\end{aligned}
$$

o que mostra que a densidade a posteriori é própria.

\section{Densidades a posteriori marginais pelo método de Laplace}

$\mathrm{Na}$ inferência Bayesiana as inferências sobre os parâmetros são conduzidas com base nas densidades a posteriori marginais. As marginais para os parâmetros $\lambda$ e $p$ são obtidas por integração da densidade a posteriori conjunta (6.16) em relação a $p$ e $\lambda$, respectivamente. Como em geral essas integrais não permitem soluções explicitas, utilizaremos o método de Laplace (veja, Tierney e Kadane, 1986).

A partir da densidade a posteriori conjunta dada em (6.16), a densidade marginal para $\lambda$ pode ser escrita na forma:

$$
\pi(\lambda \mid D) \propto \lambda^{r-1} \mathrm{e}^{-\lambda R} \int_{0}^{1} p^{r}(1-p)^{-1 / 2} \prod_{i=1}^{n}\left(1-p+p \mathrm{e}^{-\lambda t_{i}}\right)^{1-\delta_{i}} d p
$$

Portanto, utilizando a aproximação de Laplace em (6.17), obtem-se a densidade a posteriori marginal aproximada para $\lambda$ dada por

$$
\pi(\lambda \mid D) \propto \sigma \lambda^{r-1} \exp \{-\lambda R-n h(\lambda, \hat{p})\}
$$


onde

$$
-n h(\lambda, p)=r \log p-\frac{1}{2} \log (1-p)+\sum_{i=1}^{n}\left(1-\delta_{i}\right) \log \left(1-p+p e^{-\lambda t_{i}}\right),
$$

$\sigma=\left\{\frac{\partial^{2} h(\lambda, \hat{p})}{\partial p^{2}}\right\}^{-1 / 2}$ e $\hat{p}$ maximiza (6.19) para cada valor de $\lambda$ fixo.

Pode-se mostrar que a densidade a posteriori marginal aproximada pelo método de Laplace para $p$ é dada por

$$
\pi(p \mid D) \propto \sigma^{*} p^{r}(1-p)^{-1 / 2} \exp \left\{-n h^{*}(\hat{\lambda}, p)\right\}
$$

onde

$$
-n h^{*}(\lambda, p)=(r-1) \log \lambda-\lambda R+\sum_{i=1}^{n}\left(1-\delta_{i}\right) \log \left(1-p+p e^{-\lambda t_{i}}\right),
$$

$\sigma^{*}=\left\{\frac{\partial^{2} h^{*}(\hat{\lambda}, p)}{\partial p^{2}}\right\}^{-1 / 2}$ e $\hat{\lambda}$ maximiza (6.21) para cada valor de $p$ fixo.

\section{Análise Bayesiana usando método MCMC}

Um método alternativo ao método de Laplace para a obtenção das densidades marginais de $\lambda$ e $p$ é dado pela utilização dos métodos de simulação via cadeia de Markov, como o Amostrador de Gibbs e o algoritmo de Metropolis-Hasting que sào técnicas para gerar variáveis aleatórias correspondem as distribuições marginais indiretamente, isto é, estes métodos permitem gerar variáveis aleatórias correspondentes a uma distribuição marginal sem ter que calcular esta densidade. Assim, através da simulação de uma amostra da densidade a posteriori suficientemente grande pode-se calcular os momentos de interesse e outras características de interesse desta distribuição.

Considera-se as seguintes densidades a priori para $\lambda$ e $p$ :

$$
\begin{aligned}
& \lambda \sim \Gamma\left(a_{1}, b_{1}\right), \quad \text { onde } a_{1} \text { e } b_{1} \text { são conhecidos, } \\
& p \sim \operatorname{Beta}\left(a_{2}, b_{2}\right), \quad \text { onde } a_{2} \text { e } b_{2} \text { são conhecidos, }
\end{aligned}
$$

onde $\Gamma\left(a_{1}, b_{1}\right)$ denota a função de distribuição gama com média $\frac{a_{1}}{b_{1}}$ e variância $\frac{a_{1}}{b_{1}^{2}}$ e $\operatorname{Beta}\left(a_{2}, b_{2}\right)$ denota a distribuição beta e $a_{1}, b_{1}, a_{2}$ e $b_{2}$, são constantes conhecidas basea- 
das na informação a priori do especialista e da análise preliminar dos dados. Assumindo independência entre os parâmetros a densidade a priori conjunta é dada por:

$$
\pi(\lambda, p) \propto p^{a_{2}-1}(1-p)^{b_{2}-1} \lambda^{a_{1}-1} \exp \left\{-b_{1} \lambda\right\}
$$

onde $\lambda>0$ e $0<p<1$. Observa-se que se $a_{1}=b_{1}=0, a_{2}=0$ e $b_{2}=1 / 2$ obtem-se a densidade a priori não informativa de Jeffreys dada em (6.15). Para $a_{1}=b_{1}=0$, $a_{2}=b_{2}=1$, tem-se o caso da priori uniforme própria para $p$, mas, a densidade conjunta a priori dada em (6.23) se reduz a uma densidade a priori não informativa, ou seja,

$$
\pi(\lambda, p) \propto \frac{1}{\lambda}, \lambda>0, \quad 0 \leq p \leq 1
$$

Considerando a densidade a priori dada em (6.23) e a função de verossimilhança (6.2), a densidade conjunta a posteriori de $\lambda$ e $p$ é dada por

$$
\pi(\lambda, p \mid D) \propto p^{r+a_{2}-1}(1-p)^{b_{2}-1} \lambda^{r+a_{1}-1} e^{-\left(R+b_{1}\right) \lambda} \prod_{i=1}^{n}\left(1-p+p e^{-\lambda t_{i}}\right)^{1-\delta_{i}},
$$

onde $D$ denota o conjunto de dados observados.

Consideramos a seguir o caso da densidade a priori própria não informativa para $p$.

Teorema 6.3.2 Se a densidade conjunta a priori é dada por (6.24) então a densidade conjunta a posteriori de $\lambda$ e p, parâmetros do modelo de mistura exponencial, é dada por

$$
\pi(\lambda, p \mid D) \propto p^{r} \lambda^{r-1} e^{-R \lambda} \prod_{i=1}^{n}\left(1-p+p e^{-\lambda t_{i}}\right)^{1-\delta_{i}},
$$

que é própria.

A demostração deste teorema é similar a do teorema 6.3.1.

A partir da densidade conjunta a posteriori de $\lambda$ e $p$ em (6.25) podemos mostrar que as densidades a posteriori condicionais para o algoritmo de Gibbs são dadas por

$$
\pi(p \mid \lambda, D) \propto p^{r+a_{2}-1}(1-p)^{b_{2}-1} \prod_{i=1}^{n}\left(1-p+p e^{-\lambda t_{i}}\right)^{1-\delta_{i}}
$$




$$
\pi(\lambda \mid p, D) \propto \lambda^{r+a_{1}-1} e^{-\lambda\left(R+b_{1}\right)} \prod_{i=1}^{n}\left(1-p+p e^{-\lambda t_{i}}\right)^{1-\delta_{i}}
$$

Observe que (6.27) e (6.28) não são padrão pelo que faremos uso do algoritmo de MetropolisHasting para obter as amostras das densidades marginais de $\lambda$ e $p$. O método de Laplace também pode ser usado para se obter densidades marginais aproximadas para $\lambda$ e $p$ a partir da densidade conjunta (6.25).

\subsubsection{Estudo de simulação}

Com a finalidade de comparar a eficiência do estimador de máxima verossimilhança (EMV) e o estimador Bayesiano, um estudo de simulação foi realizado, baseado no modelo de sobrevivência por longo periodo. Considerou-se a variável aleatória $b_{i}$ que indica se o indivíduo está em risco ou não, tem distribuição Bernoulli $(1, p) \operatorname{com}(p=0.2,0.4,0.6,0.8)$; que é a proporção de indivíduos em risco. Para os indivíduos em riscos, simulou-se os tempos de falha, $t_{i}^{*}$ de uma distribuição exponencial com média um e para os tempos de censura simulou-se uma variável aleatória uniforme no intervalo $(0,5)$. O estimador Bayesiano considerado é a moda da densidade a posteriori conjunta de $\lambda$ e $p$ dada em (6.16) e da densidade a posteriori dada no teorema 6.3.2, denotadas estas modas por $B$ e $B_{I}$, respectivamente. Várias combinações de tamanhos amostrais $(n=30,50,100)$ são consideradas. Mil réplicas foram geradas para cada simulação. Na tabela 6.3 , apresentase as médias e o erro quadrático médio (EQM) dos estimadores. Observamos que para $n$ pequeno o estimador de Bayes de $\lambda$ é melhor, enquanto que os estimadores para $p$ não diferem muito. 
Tabela 6.3: Médias e erros quadráticos médios dos estimadores de máxima verossimilhança e dos estimadores Bayesianos, para 1.000 réplicas com diferentes tamanhos amostrais com $\lambda=1$, do modelo de sobrevivência com sobreviventes por longo período.

\begin{tabular}{|c|c|c|c|c|c|c|}
\hline & & & \multicolumn{2}{|c|}{ Média } & \multicolumn{2}{|c|}{ EQM } \\
\hline$p$ & \multicolumn{2}{|l|}{$n$} & $\hat{\lambda}$ & $\dot{p}$ & $\hat{\lambda}$ & $\hat{p}$ \\
\hline \multirow[t]{3}{*}{0.2} & \multirow[t]{3}{*}{30} & MV & 2.0746 & 0.2189 & 7.0259 & 0.0068 \\
\hline & & B & 1.3976 & 0.2403 & 1.8663 & 0.0094 \\
\hline & & $B_{I}$ & 1.5611 & 0.2284 & 3.6345 & 0.0081 \\
\hline & \multirow[t]{3}{*}{50} & MV & 1.4773 & 0.2041 & 0.7541 & 0.0044 \\
\hline & & B & 1.1264 & 0.2128 & 0.3611 & 0.0053 \\
\hline & & $B_{I}$ & 1.1384 & 0.2169 & 0.3613 & 0.0053 \\
\hline & \multirow[t]{3}{*}{100} & MIV & 1.1436 & 0.1993 & 0.1744 & 0.0021 \\
\hline & & B & 0.9945 & 0.2149 & $0.15 i 4$ & 0.0028 \\
\hline & & $B_{I}$ & 0.9806 & 0.2118 & 0.1313 & 0.0028 \\
\hline \multirow[t]{3}{*}{0.4} & \multirow[t]{3}{*}{30} & IV & 1.1453 & 0.4365 & 0.3376 & 0.0182 \\
\hline & & B & 1.0774 & 0.4497 & 0.2286 & 0.0162 \\
\hline & & $B_{I}$ & 1.1469 & 0.4150 & 0.2887 & 0.0109 \\
\hline & \multirow[t]{3}{*}{50} & MV & 1.0789 & 0.4199 & 0.1432 & 0.0085 \\
\hline & & B & 0.9892 & 0.4349 & 0.1120 & 0.0086 \\
\hline & & $B_{I}$ & 0.9898 & 0.4177 & 0.1170 & 0.0072 \\
\hline & \multirow[t]{3}{*}{100} & MV & 1.0572 & 0.4090 & 0.0733 & 0.0037 \\
\hline & & B & 0.9966 & 0.4102 & 0.0710 & 0.0044 \\
\hline & & $B_{I}$ & 0.9699 & 0.4172 & 0.0644 & 0.0047 \\
\hline \multirow[t]{3}{*}{0.6} & \multirow[t]{3}{*}{30} & MV & 1.2301 & 0.5944 & 0.1786 & 0.0108 \\
\hline & & B & 1.0496 & 0.6128 & 0.1208 & 0.0135 \\
\hline & & $B_{I}$ & 1.0944 & 0.5971 & 0.1395 & 0.0113 \\
\hline & \multirow[t]{3}{*}{50} & MV & 1.0976 & 0.6033 & 0.0805 & 0.0068 \\
\hline & & B & 0.9931 & 0.6295 & 0.0746 & 0.0089 \\
\hline & & $B_{I}$ & 0.9859 & 0.6088 & 0.0723 & 0.0070 \\
\hline & \multirow[t]{3}{*}{100} & MV & 1.0380 & 0.6036 & 0.0444 & 0.0039 \\
\hline & & B & 0.9897 & 0.6154 & 0.0149 & $0.004 \tau$ \\
\hline & & $B_{I}$ & 0.9735 & 0.6110 & 0.0393 & 0.0044 \\
\hline \multirow[t]{3}{*}{0.8} & \multirow[t]{3}{*}{30} & MV & 1.2375 & 0.7468 & 0.1294 & 0.0101 \\
\hline & & B & 1.0896 & 0.7858 & 0.0820 & 0.0079 \\
\hline & & $B_{I}$ & 1.1406 & 0.7606 & 0.1028 & 0.0078 \\
\hline & \multirow[t]{3}{*}{50} & MV & 1.1248 & 0.7815 & 0.0572 & 0.0049 \\
\hline & & B & 1.0384 & 0.8080 & 0.0457 & 0.0052 \\
\hline & & $B_{I}$ & 1.0437 & 0.7873 & 0.0463 & 0.0043 \\
\hline & \multirow[t]{3}{*}{100} & MV & 1.0474 & 0.7982 & 0.0273 & $0.002 \tau$ \\
\hline & & B & 1.0027 & 0.8121 & 0.0262 & 0.0032 \\
\hline & & $B_{I}$ & 0.9913 & 0.8057 & 0.0233 & 0.0030 \\
\hline
\end{tabular}




\subsection{Teste de hipóteses Bayesiano}

Na seção 6.2 considerou-se um teste de hipóteses para verificar se a proporção de indivíduos curados de certa enfermidade é nula ou não (veja as hipóteses em (6.8)) e verificou-se também se as proporções de indivíduos curados de alguma enfermidade sobmetidos aos diferentes tratamentos são as mesmas (veja as hipóteses em (6.11) ). Nesta seção testa-se estas hipóteses considerando-se uma perpectiva Bayesiana.

Conforme vimos no capítulo 1, uma alternativa Bayesiana para testar as hipóteses dadas em (6.8), (6.11) e (6.13), é através da determinação do fator de Bayes do modelo sob a hipótese nula ( modelo $M_{0}$ ) com respeito à hipótese alternativa ( $m o d e l o M_{1}$ ). Como foi sugerido por Geisser e Eddy (1979) e enfatizado no capítulo 1, neste trabalho utilizaremos o pseudo-fator de Bayes para testar essas hipóteses, considerando as verossimilhanças preditivas do modelos $M_{0}$ e $M_{1}$, e essas verossimilhanças são obtidas como o produto das densidades preditivas condicionais ordenadas (CPO) definadas por

$$
f\left(t_{i} \mid D_{(r)}\right)=\int f\left(t_{i} \mid \theta, D_{(r)}\right) \pi\left(\theta \mid D_{(r)}\right)
$$

onde $D_{(r)}=\left(t_{1}, \ldots, t_{i-1}, t_{i+1}, t_{i+1}, \ldots, t_{n}\right.$.). O pseudo-fator de Bayes do modelo $M_{0} \mathrm{com}$ respeito ao modelo $M_{1}$ é definido por

$$
P S F B_{01}=\frac{\prod_{i=1}^{n} f\left(t_{i} \mid D_{(r)}, M_{0}\right)}{\prod_{i=1}^{n} f\left(t_{i} \mid D_{(r)}, M_{1}\right)},
$$

onde $f\left(t_{i} \mid D_{(i)}, M_{i}\right), i=0,1$ é a verossimilhança preditiva do modelo sob $H_{i}, i=0,1$. Como nos capítulos anteriores, se PSF $B_{01}<1$ consideramos $M_{1}$ como o melhor modelo. Portanto, rejeita-se $H_{0}$.

\subsubsection{Teste para $H_{0}: p=1$}

Nesta subseção testa-se as hipóteses (6.8) da seção 6.2, através de uma perspectiva Bayesiana, utilizando-se o pseudo-fator de.Baves. Para isto determina-se as verossimilhanças preditivas sob as hipóteses $H_{0}$ e $H_{1}$ dadas em (6.S). 
Dado $D=\left(t_{1}, \ldots, t_{n}\right)$, uma amostra aleatória, a função de verossimilhança para $\theta=(\lambda, 1)^{\prime}$ sob $H_{0}$ em (6.8) é dada por

$$
L_{n}\left(\lambda \mid M_{0}\right)=\lambda^{r} \exp \left\{-\lambda \sum_{i=1}^{n} t_{i}\right\}
$$

onde $r=\sum_{i=1}^{n} \delta_{i}$. Considera-se a seguinte densidade a priori para $\lambda$ dada por

$$
\lambda \sim \Gamma(a, b) \text { onde } a \text { e } b \text { são conhecidos, }
$$

e $\Gamma(a, b)$ representa a distribuição gama com média $a / b$ e variância $a / b^{2}$.

Usando (6.31), (6.32) e o teorema de Bayes pode-se mostra que as densidade a posteriori dos parâmetros do modelo $M_{0}$ é dada por

$$
\lambda \mid D, M_{0} \sim \Gamma\left(r+a, \sum_{i=1}^{n} t_{i}+b\right) .
$$

A verossimilhança preditiva sob $H_{0}$ (modelo $\left.M_{0}\right)$ é dada por

$$
f\left(D \mid M_{0}\right)=\frac{\Gamma(r+a)}{\left(\sum_{i=1}^{n} t_{i}+b\right)^{r+a}}
$$

onde $\Gamma($.$) é a função gamma.$

A função de verossimilhança para $\theta=(\lambda, p)^{\prime}$ sob $H_{1}$ (modelo $M_{1}$ ) é dada em (6.2) e a densidade a posteriori conjunta do modelo $M_{1}$ com densidades a priori independentes para $\lambda$ e $p$ dada em (6.22) é portanto dada por

$$
\pi\left(\lambda, p \mid D, M_{1}\right) \propto p^{r+a_{2}-1}(1-p)^{b_{2}-1} \lambda^{r+a_{1}-1} e^{-\left(R+b_{1}\right) \lambda} \prod_{i=1}^{n}\left(1-p+p e^{-\lambda t_{i}}\right)^{1-\delta_{i}} .
$$

Sob a hipótese $H_{1}$ não é possível obter uma expressão analítica para a verossimilhança preditiva como ocorre sob $H_{0}$. Uma estimativa da verossimilhança preditiva sob $H_{1}$ (modelo $M_{1}$ ) pode ser obtida por integração de Monte Carlo com amostras a posteriori de $\lambda$ e $p$. Isto é, podemos determinar a densidade preditiva de validação cruzada $f\left(t_{r} \mid D_{(r)}, M_{1}\right)$. 
Vimos que uma estimativa desta densidade é a média harmônica das componentes da função de verossimilhança avaliadas na amostra a posteriori (veja, capítulo 1), isto é,

$$
\hat{f}\left(t_{r} \mid D_{(r)}, M_{1}\right)=B\left(\sum_{i=1}^{B}\left[\left(p^{(i)} \lambda^{(i)} e^{-\lambda^{(i)} t_{r}}\right)^{\delta_{r}}\left(1-p^{(i)}+p^{(i)} e^{-\lambda^{(i)} t_{r}}\right)^{1-\delta_{r}}\right]^{-1}\right)^{-1},
$$

onde $B$ é o tamanho da amostra a posteriori de $\lambda$ e $p$, obtida utilizando o algoritmo de Gibbs com Metropolis-Hastings. Logo, uma estimativa da verossimilhança preditiva é dada por $\hat{f}\left(D \mid M_{1}\right)=\prod_{r=1}^{n} \hat{f}\left(t_{r} \mid D_{(r)}, M_{1}\right)$.

Seguindo o procedimento proposto por Gelfand e Dey (1994), uma estimativa alternativa a (6.36) é obtida utilizando-se o método de Laplace. Considere a definição da densidade de validação cruzada dada em (6.29)

$$
f\left(t_{r} \mid D_{(r)}, M_{1}\right)=\frac{\int L_{n}\left(\lambda, p \mid D \cdot M_{1}\right) \pi\left(\lambda, p \mid M_{1}\right) d \lambda d p}{\int L_{n-1}\left(\lambda, p \mid D_{(r)}, M_{1}\right) \pi\left(\lambda, p \mid M_{1}\right) d \lambda d p}
$$

onde $L_{n}\left(\lambda, p \mid D, M_{1}\right)$ é função de verossimilhança dada em (6.2) para o modelo $M_{1}$ dadas $n$ observações, $L_{n-1}\left(\lambda, p \mid D, M_{1}\right)$ é a função de verossimilhança dada em (6.2) para o modelo $M_{1}$ dadas $n-1$ observações (sem a observação $t_{r}$ ) e $\pi\left(\lambda, p \mid M_{1}\right)$ é a densidade a priori do modelo $M_{1}$.

Usando a aproximação de Laplace no numerador e denominador em (6.37) obtem-se que

$$
\hat{f}\left(t_{r} \mid D_{(r)}, M_{1}\right)=\left\{\frac{\Sigma}{\Sigma^{*}}\right\}^{1 / 2} \exp \left\{-\left(n h^{*}(\hat{\lambda}, \hat{p})-n h(\tilde{\lambda}, \tilde{p})\right)\right\}
$$

onde $(\hat{\lambda}, \hat{p})$ é o valor que maximiza

$$
-n h^{*}(\lambda, p)=\log L_{n}\left(\lambda, p \mid D, M_{1}\right)+\log \pi\left(\lambda, p \mid M_{1}\right)
$$

$\Sigma^{*}=\operatorname{det}\left(n D^{2}(\hat{\lambda}, \hat{p})\right)$, sendo $D^{2}(\hat{\lambda}, \hat{p})$ a matriz hessiana de $h(\lambda, p)$ calculada em $(\hat{\lambda}, \hat{p})$ e $(\tilde{\lambda}, \tilde{p})$ o valor que maximiza

$$
-n h(\lambda, p)=\log L_{n-1}\left(\lambda, p \mid D, M_{1}\right)+\log \pi\left(\lambda, p \mid M_{1}\right),
$$

$\Sigma=\operatorname{det}\left(n D^{2}(\tilde{\lambda}, \tilde{p})\right)$, sendo $D^{2}(\tilde{\lambda}, \tilde{p})$ a matriz hessiana de $h(\lambda, p)$ avaliada em $(\tilde{\lambda}, \tilde{p})$. 


\subsubsection{Estudo de simulação}

Nesta subseção apresenta-se um estudo de simulação para verificar o comportamento do pseudo fator de Bayes para discriminar entre os modelos sob $H_{0}$ e sob $H_{1}$. As verossimilhanças preditivas foram approximadas utilizando-se o método de Laplace e o método MCMC. Para este último método considerou-se 50 amostras para cada tamanho amostral que levaram a rejeição pelo método Laplace. O estudo de simulação é baseado num modelo exponencial com média um para os tempo de vida e a variável aleatória dos tempos de censura $c_{i}$ são independentes dos tempos de vida $t_{i}^{*}$, tendo distribuição uniforme no intervalo $(0,5)$. Para o modelo sob $H_{1}$ com a priori dada em (6.22), tomamos $a_{1}=b_{1}=0$ e $a_{2}=b_{2}=1$, enquanto que sob $H_{0}$, para a priori (6.32) tomamos $a=b=0$. Várias combinações de tamanhos amostrais $(n=20,30.50,100)$ foram consideradas no estudo. Mil amostras foram geradas para cada simulação. Na tabela 6.4, apresenta-se número de vezes que a hipóteses foi rejeitada considerando-se os pseudo-fator de Bayes do modelo sob $H_{0}$ com respeito o modelo sob $H_{1}$. Consideramos que $H_{0}$ é rejeitado quando $P S B F_{01}<1$. Por exemplo, $\operatorname{com} n=20$, de 50 amostras apresentando rejeição com o método de Laplace apenas 47 apresentam rejeição com o método MCMC.

Tabela 6.4: Número de vezes que $H_{0}: p=1$ foi rejeitada em 1000 amostras

\begin{tabular}{|l|llll|}
\hline \multirow{2}{*}{ PSFB } & \multicolumn{4}{|c|}{$n$} \\
\cline { 2 - 5 } & 20 & 30 & 50 & 100 \\
\hline Laplace & 142 & 141 & 124 & 98 \\
MCMC & $47(50)$ & $46(50)$ & $44(50)$ & $42(50)$ \\
\hline
\end{tabular}

\subsubsection{Comparação de proporções de sobreviventes}

Suponhamos agora que $G$ tratamentos diferentes foram aplicados a $n$ indivíduos de modo que, cada um destes tratamentos foi aplicado a $n_{g}$ indivíduos escolhidos alea- 
toriamente, $g=1, \ldots, G$. Queremos verificar se as proporções de indivíduos que são curados pelos diferentes tratamentos é a mesma, isto é, testar as hipóteses dada em (6.11). Para isto, observou-se os tempos de falha ou censura $t_{11}, \ldots, t_{n_{1} 1}, \ldots, t_{1 G}, \ldots, t_{n_{G} G}$, $\delta_{11}, \ldots, \delta_{n_{1} 1}, \ldots, \delta_{1 G}, \ldots, \delta_{n_{G} G}$, sendo, $t_{i g}=\min \left(t_{i g}^{*}, c_{i g}\right)$, onde, $t_{i g}^{*}$ denota o tempo de falha do $i$-ésimo indivíduo em risco, devido ao $g$-ésimo tratamento (grupo) $g=1, \ldots, G$ e $i=1, \ldots, n_{g}$, com distribuição exponencial com média $\frac{1}{\lambda_{g}}, c_{i g}$ é o tempo de censura do $i$-ésimo indivíduo no $g$-ésimo grupo e $\delta_{i g}$ é o indicador de censura $\left(\delta_{i g}=1\right.$, se ocorre falha no $i$-ésimo indivíduo e $g$-ésimo tratamento (grupo) e $\delta_{i g}=0$ caso contrário).

Como na seção anterior utiliza-se o fator de Bayes para testar a hipótese dada em (6.11). Para isto, determina-se as estimativas das verossimilhanças preditivas sob a hipótese nula $H_{0}: p_{1}=\ldots, p_{G}=p$ (modelo $M_{0}$ ) e sob $H_{1}: p_{j} \neq p$ para algum $j=1 \ldots G$ (modelo $M_{1}$ ), utilizando-se amostras a posteriori de cada modelo.

\section{Densidade conjunta a posteriori dos parâmeros do modelo $M_{1}$}

A função de verossimilhança para $p_{1}, \ldots, p_{G}, \lambda_{1}, \ldots, \lambda_{G}$ sob o modelo $M_{1}$ considerando que as $G$ amostras são independentes é dada por

$$
\begin{aligned}
L\left(\lambda_{1}, \ldots, \lambda_{G}, p_{1}, \ldots, p_{G}\right)= & \prod_{j=1}^{G}\left(\lambda_{j} p_{j}\right)^{\delta^{\cdot j}} \exp \left\{-\sum_{j=1}^{G} \lambda_{j} T_{j}\right\} \\
& \times \prod_{j=1}^{G} \prod_{i=1}^{n_{j}}\left(1-p_{j}+p_{j} \epsilon^{-\lambda_{j} t_{i j}}\right)^{1-\delta_{i j}},
\end{aligned}
$$

onde $\delta_{. j}=\sum_{i=1}^{n_{j}} \delta_{i j}$ e $T_{j}=\sum_{i=1}^{n_{j}} \delta_{i j} t_{i j}$

Considere as seguintes densidades a priori independentes para $p_{j}$ e $\lambda_{j}$

$$
\begin{aligned}
& \lambda_{j} \sim \Gamma\left(c_{j}, d_{j}\right), \quad \text { onde } c_{j} \text { e } d_{j}, j=1, \ldots, G \text { são conhecidos, } \\
& p_{j} \sim \operatorname{Beta}\left(e_{j}, f_{j}\right), \quad \text { onde } e_{j} \text { e } b_{j}, j=1, \ldots, G, \text { conhecidos. }
\end{aligned}
$$

Pelo teorema de Bayes com (6.39) e (6.40), pode-se mostrar que a densidade a posteriori para o modelo $M_{1}$ é dada por 


$$
\begin{aligned}
\pi(\underline{\lambda}, \underline{P} \mid D) \propto & \prod_{j=1}^{G} \lambda_{j}^{c_{j}+\delta_{, j}-1} p_{j}^{e_{j}+\delta_{, j}-1}\left(1-p_{j}\right)^{f_{j}-1} \exp \left\{-\sum_{j=1}^{G} \lambda j\left(T_{j}+d_{j}\right)\right\} \\
& \times \prod_{j=1}^{G} \prod_{i=1}^{n_{j}}\left(1-p_{j}+p_{j} e^{-\lambda, t_{i j}}\right)^{1-\delta_{i j}} .
\end{aligned}
$$

onde $\underline{\lambda}^{\prime}=\left(\lambda_{1}, \ldots, \lambda_{g}\right)$ e $\underline{P}^{\prime}=\left(p_{1}, \ldots, p_{G}\right)$.

Teorema 6.4.1 Se a densidade a priori para $\theta^{\prime}=\left(\underline{P}^{\prime}, \underline{\lambda}^{\prime}\right.$ para o modelo $M_{1}$ é dada por (6.40) então a densidade a posteriori conjunta do modelo $M_{1}$ é própria.

Prova: Desde que $\left(1-p_{j}+p_{j} e^{-\lambda, t_{i j}}\right)^{1-\delta_{i j}} \leq 1$, para todo $p_{j}, j=1, \ldots, G$ e $i=1, \ldots, n_{j}$, temos então que

$$
\begin{aligned}
\int, \ldots, \int \pi(\underline{\lambda}, \underline{P} \mid D) d \underline{\lambda} d \underline{P} \leq & \prod_{j=1}^{G}\left(\int_{0}^{1} p_{j}^{e_{j}+\delta_{. j}-1}\left(1-p_{j}\right)^{f_{j}-1} d p_{j}\right) \\
& \times\left(\int_{0}^{\infty} \lambda_{j}^{c_{j}+\delta_{. j}-1} \exp \left\{-\lambda j\left(T_{j}+d_{j}\right)\right\} d \lambda_{j}\right) \\
& =\prod_{i=1}^{G} \frac{\Gamma\left(e_{j}+\delta_{. j}\right) \Gamma\left(f_{j}\right)}{\Gamma\left(e_{j}+\delta_{. j}+f_{j}\right)} \times \frac{\Gamma\left(c_{j}+\delta_{. j}\right)}{\left(T_{j}+d_{j}\right)^{c_{j}+\delta_{. j}}}<\infty .
\end{aligned}
$$

o que mostra que a densidade a posteriori do modelo $M_{1}$ é própria.

A partir da densidade a posteriori conjunta de $\theta$ sob o modelo $M_{1}$ dado em (6.41) pode-se mostrar que as condicionais completas para a implementação do algoritmo de Gibbs são dadas por

$$
\begin{aligned}
& \pi\left(\lambda_{j} \mid \underline{\lambda}_{(j)}, \underline{P}, D\right) \propto \lambda_{j}^{c-j+\delta_{, j}-1} e^{-\lambda_{j}\left(T_{j}+d_{j}\right)} \Psi\left(\lambda_{j}, p_{j}\right) \\
& \pi\left(p_{j} \mid \underline{\lambda}_{,} \underline{P}_{(j)} . D\right) \propto p_{j}^{e_{j}+\delta_{. j}-1}\left(1-p_{j}\right)^{f_{j}-1} \Psi\left(\lambda_{j}, p_{j}\right),
\end{aligned}
$$

onde $\underline{\lambda}_{(j)}=\left(\lambda_{1}, \ldots, \lambda_{j-1}, \lambda_{j+1}, \ldots, \lambda_{G}\right), \underline{P}_{(j)}=\left(p_{1}, \ldots, p_{j-1}, p_{j+1}, \ldots, p_{G}\right) \mathrm{e}$

$$
\Psi\left(\lambda_{j}, p_{j}\right)=\prod_{j=1}^{G}\left(1-p_{j}+p_{j} e^{-\lambda_{j} t_{i j}}\right)^{1-\delta_{i j}} .
$$

Observe que as densidades a posteriori condicionais em (6.42) não possuem uma distribuição padrão, pelo que faremos uso do algoritmo de Metropolis-Hasting com Gibbs para obter as amostras a posteriori. 
A estimativa da verossimilhança preditiva sob o modelo $M_{1}$ será obtida como o produto das densidades preditivas de validação cruzada e, a estimativa dessa densidade com as amostras a posteriori dos parâmetros sob o modelo $M_{1}$ é dada por

$$
\hat{f}\left(t_{r j} \mid D_{(r j)}, M_{1}\right)= \begin{cases}B\left(\sum_{k=1}^{B}\left[p_{j}^{(k)} \lambda_{j}^{(k)} \exp \left(-\lambda_{j}^{(k)} t_{r j}\right)\right]^{-1}\right)^{-1}, & \text { se } \delta_{r j}=1 \\ B\left(\sum_{k=1}^{B}\left[1-p_{j}^{(k)}\left(1+\exp \left(-\lambda_{j}^{(k)} t_{r j}\right)\right)\right]^{-1}\right)^{-1}, & \text { se } \delta_{r j}=0\end{cases}
$$

onde $\left(\lambda_{j}^{(k)}, p_{j}^{(k)}\right), k=1, \ldots, B$, é amostra a posteriori de $\lambda_{j} ; p_{j} ; B$ é o tamanho da amostra no modelo $M_{1}$ e $D_{(r j)}$ denota o conjunto de observações sem a observação correspondente ao indivíduo $r$ do grupo $j, j=1, \ldots, G$ e $r=1, \ldots, n_{j}$.

\section{Densidade conjunta a posteriori para os parâmetros sob o modelo $M_{0}$}

A função de verossimilhança para $\left(\lambda_{1}, \ldots, \lambda_{G}, p\right)$ sob $H_{0}$ (modelo $M_{0}$ ) é dada por

$$
L(\underline{\lambda}, p \mid D) \propto p^{r} \prod_{j=1}^{G} \lambda_{j}^{\delta_{j},} \exp \left\{-\sum_{i=1}^{G} \lambda_{j} T_{j}\right\} \prod_{j=1}^{G} \prod_{i=1}^{n_{j}}\left(1-p+p e^{\lambda_{j} t_{i j}}\right)^{1-\delta_{i j}},
$$

onde $r_{1}=\sum_{j=1}^{G} \sum_{i=1}^{n_{j}} \delta_{i j}$. Para os parâmetros sob o modelo $M_{0}$ consideramos a seguintes densidades a priori independentes:

$$
\begin{aligned}
\lambda_{j} & \sim \Gamma\left(g_{j}, h_{j}\right), \text { onde } g_{j} \text { e } h_{j} \text { são conhecidos } j=1, \ldots, G \\
p & \sim \operatorname{Beta}(e, w), \text { onde } e \text { e } w \text { são conhecidos. }
\end{aligned}
$$

Considerando (6.44) e (6.45) pode-se mostrar que a densidade conjunta a posteriori sob o modelo $M_{0}$ é dada por

$$
\begin{aligned}
\pi(\underline{\lambda}, p \mid D) & \propto \prod_{j=1}^{G} \lambda_{j}^{g_{j}+\delta_{, j}-1} p^{e+r_{1}-1}(1-p)^{w-1} \exp \left\{-\sum_{j=1}^{G} \lambda_{j}\left(T_{j}+h_{j}\right)\right\} \\
& \times \prod_{j=1}^{G} \prod_{i=1}^{n_{j}}\left(1-p\left(1-e^{\lambda, t_{i j}}\right)\right)^{1-\delta_{i j}} .
\end{aligned}
$$


Teorema 6.4.2 No modelo sob $H_{0}$ dada em (6.11) (modelo $M_{0}$ ), se a densidade a priori para $(\underline{\lambda}, p)$ é dada em (6.45) então a densidade a posteriori dada em (6.46) é própria.

Prova: Já que $\left.\left(1-p+p e^{\lambda, t_{i j}}\right)\right)^{1-\delta_{i j}} \leq 1$ para todo $j=1, \ldots, G$ e $i=1, \ldots, n_{j}$, temos então que

$$
\begin{aligned}
\int \ldots \int \pi(\underline{\lambda}, p \mid D) d \underline{\lambda} d p & \leq \int_{0}^{1} p^{e+r_{1}-1}(1-p)^{w-1} d p \prod_{j=1}^{G}\left(\int_{0}^{\infty} \lambda_{j}^{g_{j}+\delta_{. j}-1} e^{-\lambda_{j}\left(T_{j}+h_{j}\right)} d \lambda_{j}\right) \\
& =\frac{\Gamma\left(e+r_{1}\right) \Gamma(w)}{\Gamma\left(e+r_{1}+w\right)} \prod_{j=1}^{G} \frac{\Gamma\left(g_{j}+\delta_{. j}\right)}{\left[T_{j}+h_{j}\right]^{g_{j}+\delta_{. j}}}<\infty .
\end{aligned}
$$

O teorema 6.4.2 segue sendo válido no caso que a densidade a priori para o modelo $M_{0}$ é dada por $\pi(\underline{\lambda}, p) \propto 1 / \prod_{j=1}^{G} \lambda_{j}$. Isto é, densidades a priori não informativas independentes.

A partir da densidade conjunta a posteriori do modelo $M_{0}$ dada em (6.46), as condicionais completas para o algoritmo de Gibbs são dadas por

$$
\begin{aligned}
\pi\left(\lambda_{j} \mid \underline{\lambda}_{(j)}, p, D\right) & \propto \lambda_{j}^{g_{j}+\delta_{, j}-1} \exp \left\{-\lambda_{j}\left(T_{j}+h_{j}\right)\right\} \prod_{i=1}^{n_{j}}\left(1-p\left(1-e^{-\lambda_{j} t_{i j}}\right)\right)^{1-\delta_{i j}} \\
\pi(p \mid \underline{\lambda}, p, D) & \propto p^{e+r_{1}-1}(1-p)^{w-1} \prod_{j=1}^{G} \prod_{1=1}^{n_{j}}\left(1-p\left(1-e^{-\lambda, t_{i j}}\right)\right)^{1-\delta_{i j}} .
\end{aligned}
$$

Como as condicionais a posteriori dadas em (6.47) não são distribuições conhecidas utilizaremos a algoritmo de Metropolis-Hasting para obter as amostras a posteriori.

A estimativa da verossimilhança preditiva sob o modelo $M_{0}$ (modelo sob $H_{0}$ dada em (6.11)) será obtida como o produto da estimativas das densidades preditivas de validação cruzada ou CPO, dadas em (8.24). A estimativa dessas densidades com as amostras Gibbs dos parâmetros sob o modelo $M_{0}$ são dadas por

$$
\hat{f}\left(t_{r j} \mid D_{(r j)}, M_{0}\right)= \begin{cases}B\left(\sum_{k=1}^{B}\left[p^{(k)} \lambda_{j}^{(k)} \exp \left(-\lambda_{j}^{(k)} t_{r j}\right)\right]^{-1}\right)^{-1}, & \text { se } \delta_{r j}=1 \\ B\left(\sum_{k=1}^{B}\left[1-p^{(k)}\left(1+\exp \left(-\lambda_{j}^{(k)} t_{r j}\right)\right]^{-1}\right)^{-1},\right. & \text { se } \delta_{r j}=0\end{cases}
$$

onde $\left(\lambda_{j}^{(k)}, p^{(k)}\right)$ é a $k$ - ésima amostra Gibbs de $\lambda_{j}, p$ no modelo $M_{1}, k=1, \ldots, B$, $j=1, \ldots, G$ e $B$ é o tamanho da amostra. 
Com $(6.43)$ e (6.48) pode-se calcular o pseudo-fator de Bayes do modelo $M_{0}$ com respeito ao modelo $M_{1}$, que denotaremos por $P S F B_{01}$ e rejeita-se $H_{0}$ se $P S F B_{01}<1$.

\subsubsection{Teste para $H_{0}: p_{1}=\ldots=p_{G}=1$}

Nesta subseção testa-se as hipóteses dadas em (6.13) usando a perpespectiva Bayesiana, isto é, queremos determinar o fator de Bayes do modelo sob $H_{0}$ (modelo $M_{0}$ ) com respeito ao modelo sob $H_{1}$ (modelo $M_{1}$ ).

A função de verossimilhança para $\left(\lambda_{1}, \ldots, \lambda_{G}\right)$ sob o modelo $M_{0}$ é dada por

$$
L\left(\lambda_{1}, \ldots, \lambda_{G}\right) \propto \prod_{j=1}^{G} \lambda_{j}^{\delta_{j},} \exp \left\{-\sum_{j=1}^{G} \lambda_{j} t_{\cdot j}\right\}
$$

onde $t_{. j}=\sum_{i=1}^{n_{j}} t_{i j}$. Observe que (6.49) é a função de verossimilhança correspondente a $G$ amostras independentes do modelo de sobrevivência exponencial com média $\lambda_{j}, j=$ $1, \ldots, G$.

Vamos a considerar as seguintes densidades a priori independentes para $\lambda_{1}, \ldots, \lambda_{G}$ :

$$
\lambda_{j} \sim \Gamma\left(l_{j}, k_{j}\right), \text { onde } l_{j} \text { e } k_{j} \text { são conhecidos. }
$$

Considerando (6.49) e (6.50), pode-se mostrar que a densidade conjuta a posteriori para o modelo $M_{0}$ é dada por

$$
\pi\left(\lambda_{1}, \ldots, \lambda_{G} \mid D\right) \propto \prod_{j=1}^{G} \lambda_{j}^{l_{j}+\delta_{. j}-1} \exp \left\{-\sum_{j=1}^{G} \lambda_{j}\left(t_{. j}+k_{j}\right)\right\} .
$$

Observe que a densidade a posteriori dada em (6.51) é o produto de distruibuições gamas e daí pode-se mostrar que a verossimilhança preditiva sob o modelo $M_{0}$ é dada por

$$
f\left(D \mid M_{0}\right)=\prod_{i=1}^{G} \frac{\Gamma\left(\delta_{. j}+c_{j}\right)}{\left(t_{. j}+d_{j}\right)^{\delta_{, j}+c_{j}}}, .
$$

onde $\Gamma($.$) denota a função gama.$ 
A densidade a posteriori sob a hipóteses alternativa $H_{1}$ dada em (6.13) é similar à densidade dada em (6.41) se consideramos em nossa hipótese $H_{1}: p_{g} \neq 1$, para $g=$ $1, \ldots, G$. Logo, a verossimilhança preditiva para este modelo pode ser obtida como em (6.43).

\section{Estudo de simulação}

Nesta subseção apresenta-se um estudo de simulação para verificar o comportamento do pseudo fator de Bayes para discriminar entre os modelos sob $H_{0}: p_{1}=p_{2}=1$ (modelo $M_{0}$ ) e sob $H_{1}: p_{g} \neq 1$ (modelo $M_{1}$ ), $g=1,2$, como dado em (6.13). A aproximação das verossimilhanças preditivas foi obtida utilizando-se os métodos de Laplace e MCMC. Para este último método considerou-se 50 réplicas para cada tamanho amostral que apresentaram rejeição pelo método de Laplace. O estudo de simulação é baseado em dois modelos exponenciais com média um para os tempos de vida e a variável aleatória dos tempos de censura $c_{i}$ são independentes dos tempo de vida $t_{i}^{*}$ tendo sido gerados como uma variável aleatória uniforme no intervalo $(0,5)$. Considerou-se para os parâmetros da densidade a priori para o modelo $M_{1}$ dado em $(6.40)$, com $c_{j}=d_{j}=0$ e $e_{j}=f_{j}=1, j=1,2 \mathrm{e}$ a densidade a priori para o modelo $M_{0}$ dado (6.50) $\operatorname{com} l_{j}=k_{j}=0, j=1,2$. Várias combinações de tamanhos amostrais $\left(n_{1}=n_{2}=n=20,30,50,100\right)$ foram consideradas no estudo. Mil réplicas foram geradas para cada simulação. Na tabela 6.5 , apresenta-se o número de vezes que a hipótese foi rejeitada. Rejeita-se $H_{0}$ se o pseudo-fator de Bayes do modelo $M_{0}$ (sob $\left.H_{0}\right)$ com respeito o modelo $M_{1}$ ( sob $\left.H_{1}\right)$ é menor que $u m$. Para $n=20$, por exemplo, das 50 amostras onde se rejeita $H_{0}$ com o método de Laplace, rejeita-se apenas 45 com o método MCMC. 
Tabela 6.5: Número de vezes que $H_{0}: p_{1}=p_{2}=1$ foi rejeitada em 1000 réplicas

\begin{tabular}{|l|llll|}
\hline \multirow{2}{*}{ PSFB } & \multicolumn{4}{|c|}{$n$} \\
\cline { 2 - 5 } & 20 & 30 & 50 & 100 \\
\hline Laplace & 159 & 142 & 138 & 102 \\
MCMC & $45(50)$ & $44(50)$ & $43(50)$ & $47(50)$ \\
\hline
\end{tabular}

\subsection{Exemplo ilustrativo}

Nesta seção apresentamos um exemplo como aplicação dos procedimentos clássicos e Bayesianos, discutidos nas seções anteriores. O exemplo consiste em analisar os dados (veja tabela 6.6) obtidos por Kersey et al. (1987), os quais se referem aos tempos de recaída de pacientes de leucemia submetidos a dois tipos de transplante, "allogeneic" (Grupo 1) ou "autologous" (Grupo 2). O Grupo 1, consiste de observações de 46 pacientes, dos quais 13 correspondem a observações censuradas, enquanto, que o Grupo 2, consiste de observações de 44 pacientes dos quais 9 correspondem a observações censuradas.

\subsubsection{Análise clássica}

Nesta subseção apresenta-se uma aplicação da métodologia discutida na seção 2. Considerandose apenas os dados do Grupo 1 da tabela 6.6, são apresentados na tabela 6.7 as estimativas de máxima verossimilhança e os intervalos de confiança de $95 \%$ para $\lambda$ e $p$.

A seguir verifica-se a validade da hipótese dada em (6.\$), isto é, testa-se se existem indivíduos que não apresentam recaída após o transplante "allogeneic". O estimador de máxima verossimilhança sob $H_{0}$ em (6.8) é $\tilde{\lambda}=0,4728$, e a estatística da razão de verossimilhança dada em (6.9) resultou em $R V_{n}=22,979$. O percentil de $99 \%$ denotado por $c_{0,99}$ da distribuição dada em (6.10) é tal que

$$
\frac{1}{2}+\frac{1}{2} P\left(\chi_{(1)}^{2} \leq c_{0,99}\right)=0,99
$$


Tabela 6.6: Tempos de recaida de pacientes com leucemia

\begin{tabular}{|cccc|cccc|}
\hline \multicolumn{3}{|c}{ Grupo 1 } & \multicolumn{5}{c|}{ Grupo 2 } \\
\hline$t_{i}$ & $\delta_{i}$ & $t_{i}$ & $\delta_{i}$ & $t_{i}$ & $\delta_{i}$ & $t_{i}$ & $\delta_{i}$ \\
\hline 0.0301 & 1 & 0.9096 & 1 & 0.0575 & 1 & 0.3589 & 1 \\
0.0484 & 1 & 0.9644 & 1 & 0.1096 & 1 & 0.4027 & 1 \\
0.0630 & 1 & 1.0082 & 1 & 0.1370 & 1 & 0.4685 & 1 \\
0.0849 & 1 & 1.2822 & 1 & 0.1452 & 1 & 0.4712 & 1 \\
0.0877 & 1 & 1.3452 & 1 & 0.1479 & 1 & 0.4904 & 1 \\
0.1397 & 1 & 1.4000 & 1 & 0.1534 & 1 & 0.5178 & 1 \\
0.1616 & 1 & 1.7205 & 0 & 0.1753 & 1 & 0.5452 & 1 \\
0.1699 & 1 & 1.9890 & 0 & 0.1836 & 1 & 0.5836 & 1 \\
$0.2137^{2}$ & 1 & 2.2438 & 1 & 0.2000 & 1 & 0.6110 & 1 \\
0.2164 & 1 & 2.5068 & 0 & 0.2082 & 1 & 0.6137 & 1 \\
0.2384 & 1 & 2.6466 & 0 & 0.2164 & 1 & 0.7589 & 1 \\
0.2712 & 1 & 3.0384 & 0 & 0.2219 & 1 & 1.9836 & 0 \\
0.2740 & 1 & 3.1726 & 0 & 0.2411 & 1 & 1.9973 & 0 \\
0.3863 & 1 & 3.4411 & 1 & 0.2603 & 1 & 2.0110 & 1 \\
0.4384 & 1 & 4.4219 & 0 & $0.2685^{2}$ & 1 & 2.8849 & 0 \\
0.4548 & 1 & 4.4356 & 0 & 0.2712 & 1 & 2.9973 & 0 \\
0.5918 & 1 & 4.5863 & 0 & 0.2849 & 1 & 3.2658 & 0 \\
0.6000 & 1 & 4.6904 & 0 & 0.2877 & 1 & 4.0411 & 0 \\
0.6348 & 1 & 4.7808 & 0 & 0.2904 & 1 & $4.2055^{2}$ & 0 \\
0.6849 & 1 & 4.9863 & 0 & 0.3068 & 1 & 5.0548 & 0 \\
0.7397 & 1 & 5.0000 & 0 & & & & \\
0.8575 & 1 & & & & & & \\
\hline & & & & & & & \\
\hline
\end{tabular}


Tabela 6.7: Estimativas de máxima verossimilhança do modelo de mistura exponencial

\begin{tabular}{|cccc|}
\hline & EMV & D.P & Intervalo de confiança $95 \%$ \\
\hline$\hat{\lambda}$ & 1,4332 & 0,2756 & $(0,8931 ; 1,9733))$ \\
$\hat{p}$ & 0,7289 & 0,0675 & $(0,5966 ; 0,8613)$ \\
\hline
\end{tabular}

de onde tem-se que $c_{0,99}=5,41$. Já que, $22,979>5,41$ rejeita-se $H_{0}$ ao nível de $1 \%$ de confiança, e portanto, há uma forte evidência de que $p<1$.

\section{Comparação de proporções de sobreviventes}

Com os dados da tabela 6.6, verifica-se a validade das hipóteses dadas em (6.13), isto é, testa-se se existem indivíduos que não apresentam recaida após o transplante, nos 2 grupos. Os EMV sob $H_{0}$ em (6.13) são $\tilde{\lambda}_{1}=0,47284$ e $\tilde{\lambda}_{2}=0,8026$ e os EMV sob $H_{1}$ em (6.13) é $\hat{\lambda}_{1}=1,4332, \hat{p}_{1}=0,728893, \hat{\lambda}_{2}=2,681$ e $\hat{p}_{2}=0,79638$. A estatística da razão de verosimilhança dada em (6.14), resultou em $d_{n}=62,604$. O percentil de $95 \%$ da distribuição assintótica (6.14) é $C_{0,95}=4,23$. Como $62,604>4,23$, rejeita-se $H_{0}$ ao nível de confiança de $5 \%$ e portanto, há forte evidência de que pelos menos um $p_{g}<1$, $g=1,2$.

Como a hipótese $H_{0}$ dada em (6.13) foi rejeitada verifica-se a seguir se a porcentagem de indivíduos que apresentam recaida após os transplante é a mesma em ambos grupos, isto é, testa-se as hipóteses dada em (6.11). O EMV sob $H_{0}: p_{1}=p_{2}=p$ são $\tilde{\lambda}_{1}=1,41$, $\tilde{\lambda}_{2}=2,684$ e $\tilde{p}=0,76306$. A estatística da razão de verossimilhança dada em (6.12) resultou em $R V_{n}=0,546$. O percentil de $95 \%$ da distribuição qui-quadrado com um grau de liberdade é $\chi_{1}^{2}=3,084$. Já que $0,546<3,084$ não rejeita-se $H_{0}$ ao nível de confiança de $5 \%$. 


\subsubsection{Análise Bayesiana}

Nesta subseção apresenta-se uma aplicação da metodologia Bayesiana apresentada na seção 6.3.

Densidade a posteriori marginal dos parâmetros do modelo de mistura pelo método de Laplace

Considerando os dados do Grupo 1 da tabela 6.6, apresenta-se na figura 6.1, as densidades a posteriori marginais dos parâmetros $\lambda$ e $p$ dadas em (6.18) e (6.20), aproximadas pelo método de Laplace para o modelo de sobrevivência com sobreviventes de longo período.
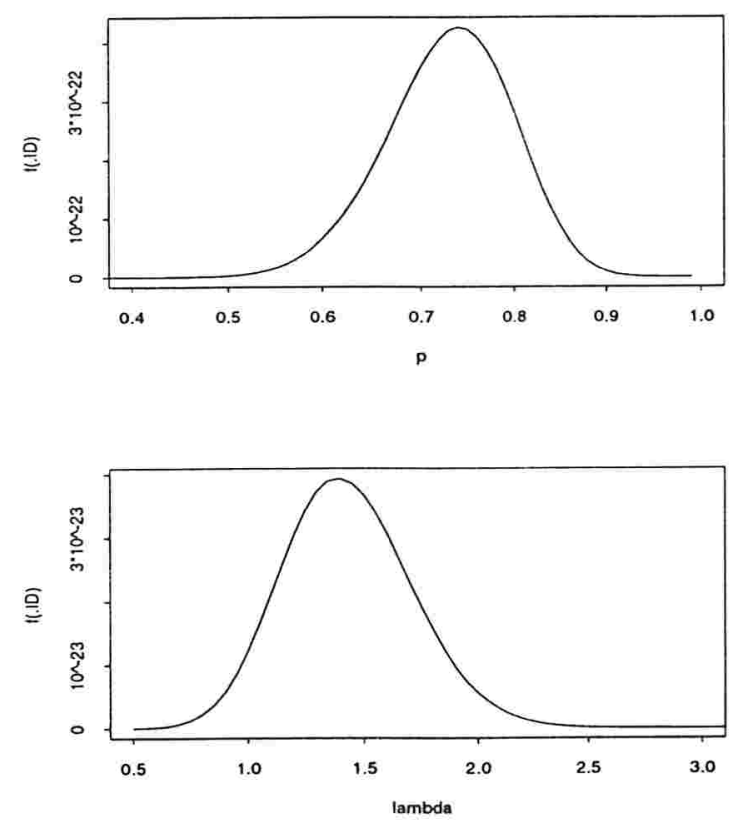

Figura 6.1: Densidades marginais a posteriori de $p$ e $\lambda$ para o modelo de mistura exponencial obtidas pelo método de Laplace

A média e desvio padrão a posteriori para $p$ e $\lambda$ aproximados pelo método de Laplace resultaram respectivamente em $E(p \mid D)=0,73033, D \cdot P \cdot(p \mid D)=0,0665, E(\lambda \mid D)=$ 1,4151 e $D \cdot P .(\lambda \mid D)=0,2776$. 


\section{Método MCMC}

Considerando os dados do Grupo 1 da tabela 6.6, para a inferência Bayesiana pelo método MCMC, consideramos as densidades a priori dada em (6.22) com $a_{1}=1, b_{1}=0,000002 \mathrm{e}$ $a_{2}=b_{2}=0,5$. Observe que a densidade a priori é não informativa. Para se obter amostras da densidade a posteriori através do algoritmo de Gibbs com Metropolis-Hasting procedese da seguinte forma. Geram-se 10 cadeias paralelas cada um com 20.000 iterações. Deprezam-se as 10.000 primeiras iterações, com objetivo de deminuir o efeito dos pontos inicias e a partir de daí escolhem-se as iterações de 10 em 10, ou seja, para cada parâmetro em cada cadeia considera-se as iterações 10.010 - ésima, . .,20.000 - ésima o qual fornece uma amostra de tamanho 20000. Para monitorar a convergência da cadeia utiliza-se a técnica de Gelman e Rubin(1992), método este que utiliza a técnica de análise de variância para verificar se mais iterações são necessárias.

$\mathrm{Na}$ tabela 6.8, apresenta-se o resumo das distribuições a posteriori (6.25) e na figura 6.2 , representa-se a densidade aproximada dos parâmetros do modelo de mistura exponencial. Também, na tabela 6.8 , tem-se o resultado da redução de escala estimada da estatística $\hat{R}$ (veja, Gelman e Rubin, 1992) para todos os parâmetros. O número de iterações considerado foi suficiente para se chegar a convergência, pois $\sqrt{\hat{R}}<1,1$ para todos os parâmetros.

Tabela 6.8: Sumário a posteriori dos parâmetros do modelo de mistura exponencial

\begin{tabular}{|cccccc|}
\hline \hline & Média & Mediana & D.P. & Intervalo de Credibilidade $95 \%$ & $\hat{R}$ \\
\hline$\lambda$ & 1,4328 & 1,4114 & 0,2780 & $(0,9312 ; 2,0222)$ & 1,000044 \\
$p$ & 0,7105 & 0,7129 & 0,06865 & $(0,5922 ; 0,8393)$ & 1,000186 \\
\hline
\end{tabular}

Da análise Bayesiana anterior pode-se concluir que não há muita diferença entre os métodos Laplace e MCMC, pois, as densidades marginais são muitos similares mesmo que as densidades a priori para as dois métodos sejam diferentes. Observe também, que as estimativas pelo método de máxima verossimilhança são muito próximas a aquelas 

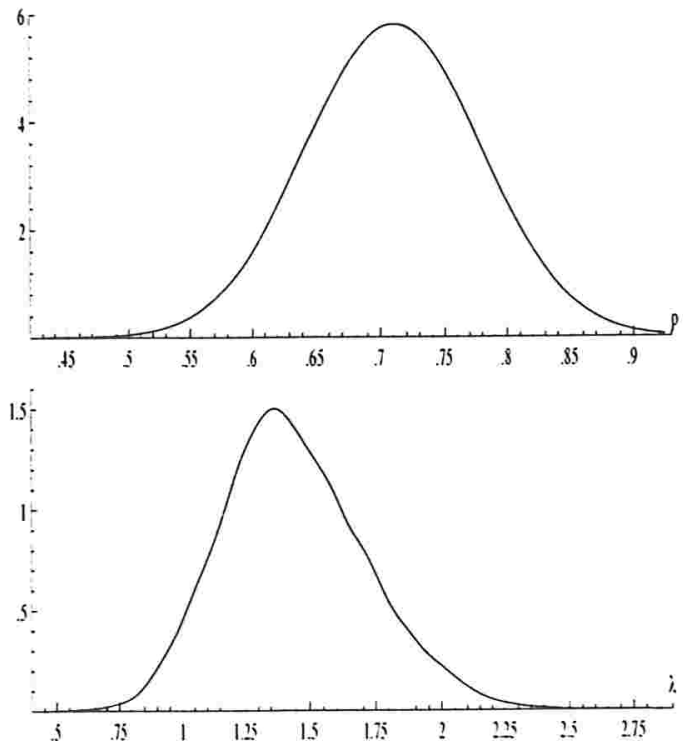

Figura 6.2: Densidades marginais a posteriori aproximadas de $p$ e $\lambda$ para o modelo de mistura exponencial pelo método MCMC

obtidas pela metodologia Bayesiana.

A seguir, vamos a testar as hipóteses dadas em (6.8), utilizando-se o pseudo-fator de Bayes. A verossimilhança preditiva sob $H_{0}$ (modelo $M_{0}$ ) dada em (6.34) considerandose a densidade a priori (6.32) com $a=1$ e $b=0.00002$ é $f\left(D \mid M_{0}\right)=1,776 \times 10^{-26}$. A estimativa da verossimilhança preditiva sob $H_{1}$ (modelo $M_{1}$ ) com as amostras a posteriori de $\lambda$ e $p$, aproximado pela densidade de validação cruzada dada em (6.36) é $\hat{f}\left(D \mid M_{1}\right)=$ $8,637 \times 10^{-22}$. O pseudo-fator de Bayes para comparar os modelos sob $M_{0}$ e $M_{1}$ é $P S F B_{01}=0,0000206$. Logo, para os dados do Grupo 1, da tabela 6.6 o modelo $M_{1}$ se ajusta melhor. Portanto, rejeita-se $H_{0}$, confirmando a conclusão obtida pelo procedimento clássico. Observamos também que o fator de Bayes enfaticamente recomenda o modelo $M_{1}$. 


\section{Comparação de proporções de sobreviventes}

Nesta subseção verifica-se inicialmente as hipóteses dada em (6.13), utilizando-se o pseudo fator de Bayes do modelo sob $H_{0}$ (modelo $M_{0}$ ) com respeito ao modelo sob $H_{1}$ (modelo $\left.M_{1}\right)$. Se a hipótese $H_{0}$ em (6.13) é rejeitada, testa-se as hipóteses dadas em (6.11), isto é, que a proporção de indivíduos que apresentam recaída é a mesma nos dois grupos.

Para o modelo sob $H_{0}$ dado em (6.13) consideramos as densidades a priori dada em (6.50) $\operatorname{com} l_{g}=1$ e $k_{g}=0,00001, g=1,2$. Neste caso a verossimilhança preditiva sob $H_{0}\left(\right.$ modelo $\left.M_{0}\right)$ dada em $(6.52)$ resultou em $f\left(D \mid M_{0}\right)=1,739 \times 10^{-45}$.

Consideramos agora o modelo sob $H_{1}$ dada em (6.13) e as densidades a priori dadas em (6.40) com $c_{g}=1$ e $d_{g}=0,00001, g=1,2$. Geramos 20 cadeias separadas cada uma com 5.000 iterações. Deprezam-se as 2.500 primeiras iterações, e daí escolhem-se as iterações de 10 em 10, fornecendo uma amostra de Gibbs de tamanho 5.000. Na tabela 6.9 , apresenta-se o resumo das distribuições a posteriori juntamente com a estimativa dos fatores de redução de escala potêncial $\hat{R}$ para todos os parâmetros que são praticamente iguais a $u m$ e na figura 6.3 , representa-se as densidades marginais aproximadas para o modelo.

Tabela 6.9: Sumário a posteriori dos parâmetros do modelo sob $H_{1}$ dada em (6.13)

\begin{tabular}{|cccccc|}
\hline \hline & Média & Mediana & D.P & Intervalo de Credibilidade $95 \%$ & $\hat{R}$ \\
\hline$\lambda_{1}$ & 1,5664 & 1,5176 & 0,3858 & $(0,98696 ; 2,38594)$ & 1,0081346 \\
$p_{1}$ & 0,72420 & 0,72736 & 0,07031 & $(0,57904 ; 0,854664)$ & 1,0000234 \\
$\lambda_{2}$ & 2,8132 & 2,7552 & 0,6077 & $(1,82204 ; 4,18017)$ & 1,0002838 \\
$p_{2}$ & 0,79729 & 0,80109 & 0,05643 & $(0,676871 ; 0,893852)$ & 1,0178026 \\
\hline
\end{tabular}

A estimativa da verossimilhança preditiva sob a hipótese $H_{1}$ (Modelo $M_{1}$ ) dada em (6.13) resultou em, $\hat{f}\left(D \mid M_{1}\right)=1,683 \times 10^{-35}$. O pseudo-fator de Bayes do modelo sob $H_{0}$ em (6.13) com respeito ao modelo sob $H_{1}$ em (6.13) é $P S F B_{01}=1,033 \times 10^{-10}<1$. Logo, rejeita-se $H_{0}$. A mesma conclusão foi obtida com o procedimento clássico, embora 

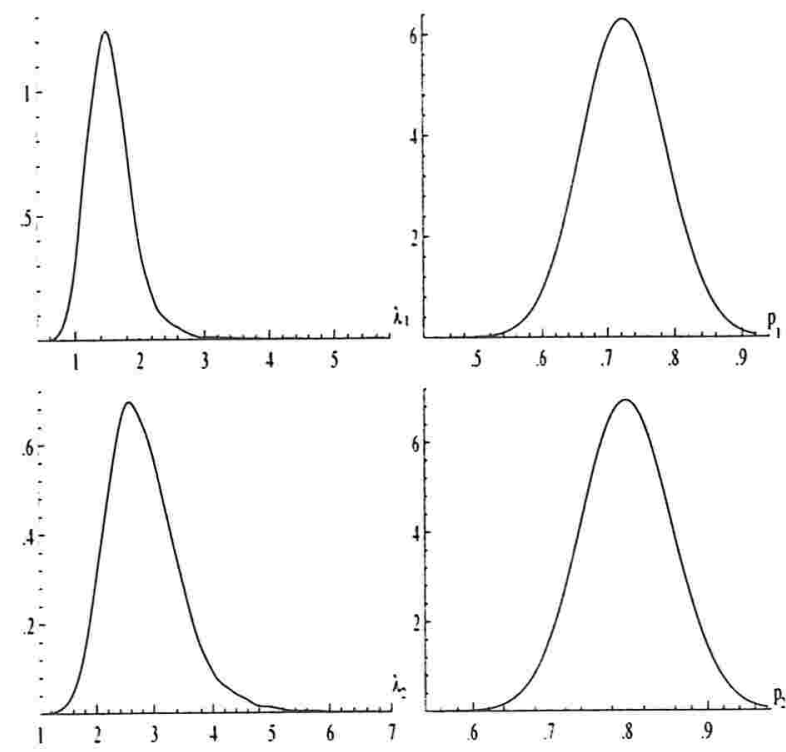

Figura 6.3: Densidades marginais a posteriori aproximadas de $\lambda_{1}, p_{1}, \lambda_{2}$ e $p_{2}$ do modelo sob $H_{1}$ em (6.13)

o pseudo-fator de Bayes parece mostrar que a diferença é mais acentuada.

A seguir vamos a testar as hipóteses dada em (6.11) de uma perspectiva Bayesiana. Considerando o modelo sob $H_{0}$ dada em (6.11) e as densidades a priori dadas em (6.45) com $g_{j}=1, h_{j}=0,00001, j=1,2$, e $e=f=1$, geramos 20 cadeias separadas com 5.000 iterações cada uma para cada parâmetro. Desprezam-se as 2.500 primeiras iterações e a partir daí escolhem-se as iterações de 10 em 10, fornecendo uma amostra de tamanho 5.000. Na tabela 6.10, apresentamos o sumário da distribuição a posteriori juntamente com o resultado do fator de redução escala potencial estimada, para todos os parâmetros e observamos que $\sqrt{\hat{R}}<1.1$ indicando a convergência e na figura 6.4 , temos as densidades marginais a posteriori aproximadas.

Com as amostras de Gibbs geradas, as estimativa da verossimilhança preditiva para o modelo sob $H_{0}$ e $H_{1}$ em $(6.11)$ são, $\hat{f}\left(D \mid M_{0}\right)=3,363 \times 10^{-35}$ e $\hat{f}\left(D \mid M_{1}\right)=1,683 \times 10^{-35}$. $\mathrm{O}$ pseudo-fator de Bayes do modelo sob $H_{0}$ com respeito ao modelo sob $H_{1}$ é $P S F B_{01}=$ 
Tabela 6.10: Sumário a posteriori dos parâmetros do modelo sob $H_{1}$ dada em (6.11)

\begin{tabular}{|cccccc|}
\hline \hline & Média & Mediana & D.P & Intervalo de Credibilidade $95 \%$ & $\hat{R}$ \\
\hline$\lambda_{1}$ & 1,5463 & 1,5036 & 0.3791 & $(0,97685 ; 2,36095)$ & 1,0063249 \\
$\lambda_{2}$ & 2,7600 & $2,725 i$ & 0.4874 & $(1,89705 ; 3,78582)$ & 1,0000291 \\
$p$ & 0,75998 & 0,76184 & 0.0458 & $(0,663455 ; 0,84437)$ & 1,0032145 \\
\hline
\end{tabular}

$1,9982>1$. Portanto, $H_{0}$ nào é rejeitada, confirmando assim a conclusão obtida pelo método clássico.

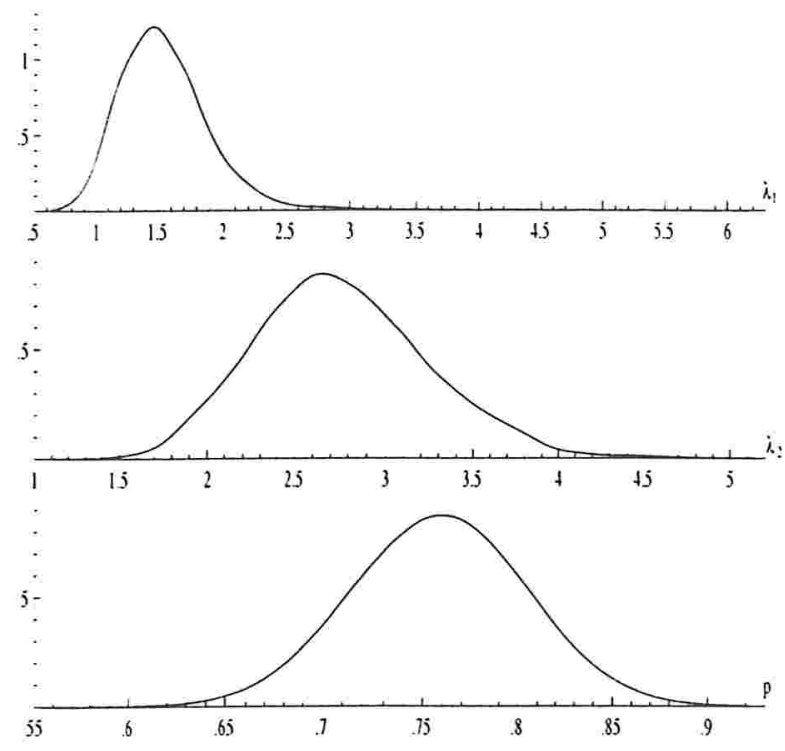

Figura 6.4: Densidades marginais a posteriori aproximadas de $\lambda_{1}, \lambda_{2}$ e $p$ do modelo sob $H_{0}$ em (6.11)

Teste para $H_{0}: p_{1}=p_{2}$ versus $H_{1}: p_{1}>p_{2}$

No caso de dois grupos $(G=2)$, muitas vezes tem-se interesse em verificar as hipóteses seguintes:

$$
H_{0}: p_{1}=p_{2} \text { versus } H_{1}: p_{1}>p_{2}
$$


Enfatizamos que não existe procedimento clássico disponivel para testar as hipóteses $H_{0}$ e $H_{1}$. Um procedimento usual em neste caso, é obtermos inferência sobre $p_{1}-p_{2}$ (ver por exemplo Box e Tiao, 1973). A função de densidade a posteriori de $p_{1}-p_{2}$ pode ser aproximada utilizando as amostras Gibbs do modelo sob $H_{1}$ em (6.11) e essa densidade é representada na figura 6.5. A probabilidade a posteriori de que $p_{1}-p_{2}>0$ é aproximadamente igual a 0, 2039 dando um forte indício de que $H_{0}$ é verdadeira. Hsu (1982) considera este enfoque em problemas de deteção de pontos de mudança. Usando o amostrador de Gibbs fica fácil implementar este procedimento, pois temos disponível amostras independentes das duas distribuições. Quando temos mais de duas proporções para comparar, poderia ser estudada o comportamento destas duas a duas, e para conclusão final poderia ser utilizado um procedimento do tipo Bonferroni.

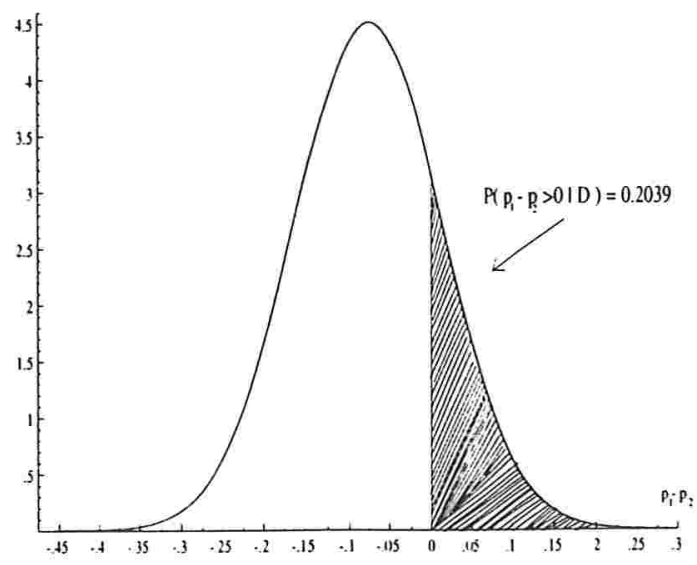

Figura 6.5: Densidade marginal a posteriori aproximado de $p_{1}-p_{2}$ 


\section{Capítulo 7}

\section{Modelos de mistura exponencial com}

covariáveis

\subsection{Introdução}

A análise de dados relacionados com os tempos de falha, onde a informação concomitante sobre os indivíduos está disponível na forma de vetores de covariáveis associadas com cada um, é de grande utilidade em muitas disciplinas tais como da medicina, engenharia e criminologia. Os métodos de covariáveis foram examinados extensivamente no contextos dos modelos de sobrevivência paramétrica para os quais a distribuição de tempos de sobrevivência depende do um vetor de covariáveis associado a cada indivíduo. Veja Feigel e Zelen (1965), Glasser (1967) e Maller (1988) para abordagens que acomodam censuras e covariáveis ao modelo de sobrevivência exponencial comum. Ghitany e Maller (1994) extenderam para o caso de covariáveis os modelos de mistura exponencial considerados por Ghitany e Maller (1992) em que a população em estudo tem uma distribuição de tempos de falha exponencial mas também contém uma proporção de imunes que são imortais no sentido de que eles não estão sujeitos a falhas. Atualmente, tais modelos de mistura com imunes e covariáveis estão em uso em muitas áreas, tais como medicina e criminologia. 
Veja, por exemplo Farewell (1982-1986), Goldman (1984), Broadhurst e Maller (1990). Análise assintótica rigorosa não é geralmente tratada nesses estudos, mas se mencionando elas, por exemplo, frequentemente assumem tipos simples de censura e covariáveis limitadas. Estas restrições não são desejáveis, nem necessárias conforme mostram Ghitany e Maller (1994) utilizando a metodolgia proposta por Fahrmmeir e Kaufmann (1985) para modelos lineares geralizados. Contudo, certas condições de regularidade ainda são requeridas para a validade dos resultados assintóticos para os estimadores de máxima verossimilhança.

$\mathrm{Na}$ formulação considerada, as covariáveis são incorporadas a um modelo de mistura exponencial com sobreviventes por longo período, considerando que a proporção de indivíduos não imunes e a taxa de falhas dependem das covariáveis e de vetores de parâmetros desconhecidos, via funções logística e loglinear. Este tipo de parametrização é comum nos modelos lineares generalizados (veja por exemplo, McCullagh e Nelder,198:3) e também garante que os parâmetros pertençam ao espaço paramétrico de interesse.

O objetivo deste capítulo é apresentar uma análise Bayesiana para os modelos de mistura exponencial com covariáveis, utilizando métodos de simulação via cadeias de Markov para determinar as distribuições a posteriori dos parâmetros envolvidos. Esta metodologia não requer nenhuma suposição ou condição de regularidade sobre o modelo, o que é uma vantagem sobre o modelo clássico.

\subsection{Análise clássica}

Nesta seção apresenta-se o modelo de mistura exponencial com covariáveis e discutimos os estimadores de máxima verossimilhança (EMV) e teste de hipóteses dos parâmetros do modelo conforme apresentado por Githany e Maller (1994). Seja $b_{i}, 1 \leq i \leq n$ uma sequência de variáveis aleatórias binárias, onde $b_{i}=1$ indica que o $i$-ésimo indivíduo está em risco, sujeito a algum tipo de falha e $b_{i}=0$ indica que o indivíduo não está em 
risco. Nos não observamos os $b_{i}$ de forma que não sabemos se um indivíduo é imune ou não, só podemos inferir a precença de imunes se muitas das observações são censuradas. Contudo, podemos ter vetores de covariáveis, $\mathrm{x}_{i} \in R^{k_{1}}, \mathrm{y}_{i} \in R^{k_{2}}$ contendo indicadores ou valores de quantidades como, por exemplo, idade, sexo, estado de saúde, disponível para cada indivíduo em consideração. Consideremos que a cováriavel $\mathrm{x}_{i}$ esta associada à taxa de falhas $\left(\lambda_{i}\right)$ e $\mathrm{y}_{i}$ à proporção de indivídous imunes, podendo inclusive serem as ser as mesmas, mas, geralmente elas diferem e podem ser consideradas com subvetores de um vetor geral de covariáveis.

Condicionalmente dado $b_{i}$, assumimos que os tempos de falha $t_{i}^{*}$ são variáveis aleatórias independentes com função de densidade de probabilidades

$$
f_{i}(t)=\lambda_{i} e^{-\lambda_{i} t}, \quad t \geq 0
$$

e a função de distribuição $F_{i}(t)=1-e^{-\lambda_{i} t}$. Assumimos que o parâmetro $\lambda_{i}$ associado ao $i$-ésimo indivíduo está relacionado com as covariáveis $\mathrm{x}_{i}$ através da relação

$$
\lambda_{i}=\exp \left(\alpha^{\prime} \mathbf{x}_{i}\right)
$$

onde $\alpha \in R^{k_{1}}$ é um vetor $k_{1}$-dimensional de parâmetros desconhecidos. Considera-se que distribuição de $b_{i}$ está relacionado com a covariável $\mathrm{y}_{i}$ através da relação

$$
p_{i}=P\left(b_{i}=1\right)=\frac{\exp \left\{\boldsymbol{\beta}^{\prime} \mathbf{y}_{i}\right\}}{1+\exp \left\{\boldsymbol{\beta}^{\prime} \mathbf{y}_{i}\right\}}=1-P\left(b_{i}=0\right)
$$

onde $\beta \in R^{k_{2}}$ é um vetor $k_{2}$-dimensional de parâmetros desconhecidos.

A transformações em (7.2) e (7.3) garantem que os $\lambda_{i}$ sejam positivos e restringe os $p_{i}$ ao intervalo $(0,1)$.

Usualmente, observamos o tempo de sobrevivência $t_{i}=\min \left(t_{i}^{*}, c_{i}\right)$, onde como antes os tempos de censura $c_{i}$ são variáveis aleatórias independentes, os quais também são independentes de $t_{i}^{*}$ e observamos $\delta_{i}=1$ se $t_{i}^{*} \leq c_{i}$ e $\delta_{i}=0$ caso contrário. 


\subsubsection{Estimadores de máxima verossimilhança}

Seja $\theta=\left(\alpha^{\prime}, \boldsymbol{\beta}^{\prime}\right)$ o vetor de parâmetros desconhecidos. A função log-verossimilhança para $\theta$ dado $\left(t_{i}, \mathrm{x}_{i}, \mathrm{y}_{i}, \delta_{i}\right), 1 \leq i \leq n$, pode ser escrita como

$$
\ell_{n}(\theta)=\sum_{i=1}^{n}\left\{\delta_{i}\left(\log p_{i}+\log \lambda_{i}-\lambda_{i} t_{i}\right)+\left(1-\delta_{i}\right)\left(1-p_{i}+p_{i} e^{-\lambda_{i} t_{i}}\right)\right\} .
$$

O EMV de $\theta$ é obtido maximizando (7.4), o que equivale a resolver o sistema de equações $S_{n}(\theta)=0$ dado a seguir.

De (7.1)-(7.2) podemos calcular o vetor das primeiras derivadas de $\partial \ell_{n}(\theta) / \partial \alpha$ e $\partial \ell_{n}(\theta) / \partial \beta$, que podemos escrever como

$$
S_{n}(\theta)=\frac{\partial}{\partial \theta} \ell_{n}(\theta)=\left(\begin{array}{c}
\frac{\partial}{\partial \alpha} \ell_{n}(\theta) \\
\frac{\partial}{\partial \beta} \ell_{n}(\theta)
\end{array}\right)=\sum_{i=1}^{n} \mathbf{X}_{i} s_{i}(\theta),
$$

onde $s_{i}(\theta)$ são vetores de dimensão 2 com componentes

$$
s_{i 1}(\theta)=\delta_{i}\left(1-\lambda_{i} t_{i}\right)-\left(1-\delta_{i}\right) \frac{p_{i} \lambda_{i} t_{i} e^{-\lambda_{i} t_{i}}}{1-p_{i}+p_{i} e^{-\lambda_{i} t_{i}}}
$$

e

$$
s_{i 2}(\theta)=\left(1-p_{i}\right)\left(\delta_{i}-\frac{\left(1-\delta_{i}\right) p_{i}\left(1-e^{-\lambda_{i} t_{i}}\right)}{1-p_{i}+p_{i} e^{-\lambda_{i} t_{i}}}\right),
$$

e $\mathrm{X}_{i}$ são matrizes não estócasticas de ordem $\left(k_{1}+k_{2}\right) \times 2$ dadas por

$$
\mathrm{X}_{i}=\left(\begin{array}{cc}
\mathrm{x}_{\mathrm{i}} & 0 \\
0 & \mathrm{y}_{\mathrm{i}}
\end{array}\right)
$$

$i=1, \ldots, n$. Como não é possível obter de modo analítico o estimador $\hat{\theta}$ que satifaz as equações acima, devemos utilizar algum método iterativo como, por exemplo, o método de Newton-Rapson para obter estimativas destes parâmetros. Esse método aparece como subrotina de alguns pacotes estatísticos tais como o Ox (veja, Doornik, 1998).

Pode-se mostrar que a matriz de segundas derivadas multiplicada por $(-1)$ pode ser escrita como

$$
\mathbf{F}_{n}(\theta)=-\frac{\partial^{2}}{\partial \theta^{2}} \ell_{n}(\theta)=-\left(\begin{array}{cc}
\frac{\partial^{2}}{\partial \alpha^{2}} \ell_{n}(\theta) & \frac{\partial^{2}}{\partial \alpha \partial \beta^{\prime}} \ell_{n}(\theta) \\
\frac{\partial^{2}}{\partial \beta \partial \alpha^{\prime}} \ell_{n}(\theta) & \frac{\partial^{2}}{\partial \beta^{2}} \ell_{n}(\theta)
\end{array}\right)
$$




$$
=\sum_{i=1}^{n} \mathrm{X}_{i} \mathcal{F}_{i}(\theta) \mathbf{X}_{i}^{\prime}
$$

onde $\mathcal{F}_{i}(\theta)$ é uma matriz aleatória simétrica de ordem $2 \times 2$ com elementos na diagonal dados por

$$
\begin{aligned}
f_{i}^{11}(\theta) & =\delta_{i} \lambda_{i} t_{i}+\left(1-\delta_{i}\right) \frac{p_{i} \lambda_{i} t_{i} e^{-\lambda_{i} t_{i}}}{1-p_{i}+p_{i} e^{-\lambda_{i} t_{i}}}\left\{1-\frac{\left(1-p_{i}\right) \lambda_{i} t_{i}}{1-p_{i}+p_{i} e^{-\lambda_{i} t_{i}}}\right\} \\
f_{i}^{22}(\theta) & =p_{i}\left(1-p_{i}\right)\left\{1-\frac{\left(1-\delta_{i}\right) e^{-\lambda_{i} t_{i}}}{1-p_{i}+p_{i} e^{-\lambda_{i} t_{i}}}\right\}
\end{aligned}
$$

e elementos fora da diagonal dados por

$$
f_{i}^{12}(\theta)=f_{i}^{21}(\theta)=p_{i}\left(1-p_{i}\right)\left(1-\delta_{i}\right) \frac{\lambda_{i} t_{i} e^{-\lambda_{i} t_{i}}}{\left(1-p_{i}+p_{i} e^{-\lambda_{i} t_{i}}\right)^{2}},
$$

$i=1, \ldots, n$. Pode-se mostrar que a matriz de informação de Fisher, $\mathbf{D}_{n}=E\left(\mathbf{F}_{n}(\theta)\right)$, satisfaz $\mathrm{D}_{n}=E\left\{S_{n}(\theta) S_{n}(\theta)^{\prime}\right\}$, de modo que de (7.9) pode-se escrever

$$
\mathbf{D}_{n}=\sum_{i=1}^{n} \mathbf{X}_{i} \mathcal{D}_{i}(\theta) \mathbf{X}_{i}^{\prime}
$$

onde $\mathcal{D}_{i}=E\left\{\mathcal{F}_{i}(\theta)\right\}, 1 \leq i \leq n$ são matrizes simétricas de ordem $2 \times 2$, com elementos $d_{i}^{r s}, r, s=1,2$ dados por

$$
\begin{aligned}
& d_{i}^{11}=p_{i} E\left\{1-e^{-\lambda_{i} c_{i}}-\frac{\left(1-p_{i}\right) \lambda_{i}^{2} c_{i}^{2} e^{-\lambda_{i} c_{i}}}{1-p_{i}+p_{i} e^{-\lambda_{i} c_{i}}}\right\}, \\
& d_{i}^{22}=p_{i}\left(1-p_{i}\right)^{2} E\left\{\frac{1-e^{-\lambda_{i} c_{i}}}{1-p_{i}+p_{i} e^{-\lambda_{i} c_{i}}}\right\}, \\
& d_{i}^{12}=d_{i}^{21}=p_{i}\left(1-p_{i}\right) E\left\{\frac{\lambda_{i} c_{i} e^{-\lambda_{i} c_{i}}}{1-p_{i}+p_{i} e^{-\lambda_{i} c_{i}}}\right\},
\end{aligned}
$$

$i=1, \ldots, n$.

\subsubsection{Inferência sobre os parâmetros $\alpha$ e $\beta$}

A inferência sobre os parâmetros do modelo de mistura exponencial com covariáveis são feitas geralmente através da normalidade assintótica dos estimadores de máxima verossimilhança. Ghitany et al. (1994) mostraram que os EMV $\hat{\alpha}$ e $\hat{\boldsymbol{\beta}}$ de $\boldsymbol{\alpha}$ e $\boldsymbol{\beta}$ sob certas 
condições de regularidade tem o seguinte comportamento, para $n \longrightarrow \infty$ :

$$
\mathrm{D}_{n}^{1 / 2}\left[\left(\begin{array}{c}
\hat{\alpha} \\
\hat{\boldsymbol{\beta}}
\end{array}\right)-\left(\begin{array}{c}
\boldsymbol{\alpha} \\
\boldsymbol{\beta}
\end{array}\right)\right] \stackrel{D}{\longrightarrow} N\left(\mathbf{0}, I_{k_{1}+k_{2}}\right)
$$

onde $\mathrm{D}_{n}^{1 / 2}$ é a matriz raíz quadrada da matriz de informação de Fisher dada em (7.14), $N\left(0, I_{k_{1}+k_{2}}\right)$ denota a distribuição normal multivariada padrão $\left(I_{k_{1}+k_{2}}\right.$ é matriz identidade de dimensão $\left.\left(k_{1}+k_{2}\right) \times\left(k_{1}+k_{2}\right)\right)$.

\subsection{Análise Bayesiana}

Na seção anterior foi discutido que a distribuição dos estimadores de máxima verossimilhança somente é conhecida assintóticamente, o que pode trazer dificuldades, uma vez que em geral, trabalha-se com amostras pequenas e moderadas. Os estudos de simulação que apresentamos na seção 6.2 ilustram alguns dificuldades que podem ocorrer. Desta forma, inferências baseadas nessas estatísticas podem não ser confiáveis. Um procedimento alternativo que pode contornar esse problema é através de utilização de métodos Bayesianos que consideram os parâmetros do modelo como variáveis aleatórias e não como constantes desconhecidas. Nesta seção desenvolve-se esse método para o caso de modelos de mistura exponencial com sobreviventes de longo período e covariáveis através de métodos computacionais.

\subsubsection{Análise Bayesiana do modelo de mistura exponencial com covariáveis usando o método MCMC}

Dada $\left(t_{i}, \delta_{i}, \mathbf{x}_{i}, \mathbf{y}_{i}\right), 1 \leq i \leq n$, amostra aleatória de tamanho $n$, a função de verossimilhança de $\theta=(\alpha, \beta)$ é dada por

$$
L(\theta)=\prod_{i=1}^{n}\left(p_{i} \lambda_{i} \exp \left\{-\lambda_{i} t_{i}\right\}\right)^{\delta_{i}}\left(1-p_{i}+p_{i} e^{-\lambda_{i} t_{i}}\right)^{1-\delta_{i}},
$$

onde $p_{i}=\frac{\exp \left\{\beta^{\prime} \mathbf{y}_{i}\right\}}{1+\exp \left\{\beta^{\prime} \mathbf{y}_{i}\right\}}$ e $\lambda_{i}=\exp \left(\alpha^{\prime} \mathbf{x}_{i}\right)$ dados em (7.2) e (7.3), respectivamente. 
Para a inferência Bayesiana consideraremos as seguintes densidades a priori para $\alpha$ e $\beta$ que consideramos independentes a priori:

$$
\begin{aligned}
& \alpha_{j} \sim N\left(\mu_{0 j}, \sigma_{0 j}^{2}\right), \quad \mu_{0 j} \text { e } \sigma_{0 j}^{2}, \mathrm{~J}=1, \ldots, k_{1} \text { conhecidos } \\
& \beta_{k} \sim N\left(\mu_{1 k}, \sigma_{1 k}^{2}\right), \quad \mu_{1 k} \text { e } \sigma_{i k}^{2}, k=1, \ldots, k_{1} \text { conhecidos }
\end{aligned}
$$

onde $N\left(\mu, \sigma^{2}\right)$ denota a distribuição normal com média $\mu$ e variância $\sigma^{2}$.

De (7.16)-(7.17) e do teorema de Bayes pode-se mostrar que a densidade a posteriori conjunta de $\alpha$ e $\beta$ é dada por

$$
\begin{aligned}
\pi(\alpha, \beta \mid D) & \propto \exp \left\{\sum_{i=1}^{n} \sum_{j=1}^{k_{1}} \delta_{i} x_{i j} \alpha_{j}-\sum_{i=1}^{n} \delta_{i} t_{i} e^{\alpha^{\prime} \mathbf{x}_{i}}-\frac{1}{2} \sum_{j=1}^{k_{1}}\left(\frac{\alpha_{j}-\mu_{0 j}}{\sigma_{0 j}}\right)^{2}-\frac{1}{2} \sum_{k=1}^{k_{2}}\left(\frac{\beta_{k}-\mu_{1 k}}{\sigma_{1 k}}\right)^{2}\right\} \\
& \times \prod_{i=1}^{n}\left(\frac{\exp \left\{\beta^{\prime} \mathbf{y}_{i}\right\}}{1+\exp \left\{\beta^{\prime} \mathbf{y}_{i}\right\}}\right)^{\delta_{i}}\left(1-\frac{\exp \left\{\beta^{\prime} \mathbf{y}_{i}\right\}}{1+\exp \left\{\beta^{\prime} \mathbf{y}_{i}\right\}}\left(1-e^{-\epsilon^{\alpha^{\prime} \mathbf{x}_{i}} t_{i}}\right)\right)^{1-\delta_{i}},
\end{aligned}
$$

onde $D$ denota o conjunto de observações.

Da clensidade a posteriori ( $(\bar{\imath} .18)$ pode-se mostrar que as densidades a posteriori condicionais para a aplicação do algoritmo de Gibbs são dadas por

$$
\begin{aligned}
& \pi\left(\alpha_{j} \mid \alpha_{(j)}, \boldsymbol{\beta}, D\right) \propto \exp \left\{\sum_{i=1}^{n} \delta_{i} x_{i j} \alpha_{j}-\sum_{i=1}^{n} \delta_{i} t_{i} e^{\alpha^{\prime} \mathbf{x}_{i}}-\frac{1}{2}\left(\frac{\alpha_{j}-\mu_{0 j}}{\sigma_{0 j}}\right)^{2}\right\} \Psi_{1}(\boldsymbol{\alpha}, \boldsymbol{\beta}), \\
& \pi\left(\beta_{k} \mid \boldsymbol{\beta}_{(k)}, \boldsymbol{\alpha}, D\right) \propto \exp \left\{-\frac{1}{2}\left(\frac{\beta_{k}-\mu_{1 k}}{\sigma_{1 k}}\right)^{2}\right\} \Psi_{2}(\boldsymbol{\alpha}, \boldsymbol{\beta}),
\end{aligned}
$$

onde $\alpha_{(j)}=\left(\alpha_{1}, \ldots, \alpha_{j-1}, \alpha_{j+1}, \ldots, \alpha_{k_{1}}\right), \boldsymbol{\beta}_{(k)}=\left(\beta_{1}, \ldots, \beta_{k-1}, \beta_{k+1}, \ldots, \beta_{k_{2}}\right)$,

$$
\Psi_{1}(\alpha, \beta)=\prod_{i=1}^{n}\left(1-\frac{\exp \left\{\beta^{\prime} y_{i}\right\}}{1+\exp \left\{\beta^{\prime} y_{i}\right\}}\left(1-e^{-e^{\alpha^{\prime} x_{i} t_{i}}}\right)\right)^{1-\delta_{i}}
$$

e

$$
\Psi_{2}(\boldsymbol{\alpha}, \boldsymbol{\beta})=\prod_{i=1}^{n}\left(\frac{\exp \left\{\beta^{\prime} \mathbf{y}_{i}\right\}}{1+\exp \left\{\beta^{\prime} \mathbf{y}_{i}\right\}}\right)^{\delta_{i}}\left(1-\frac{\exp \left\{\beta^{\prime} \mathbf{y}_{i}\right\}}{1+\exp \left\{\beta^{\prime} \mathbf{y}_{i}\right\}}\left(1-e^{-e^{\alpha^{\prime} \mathbf{x}_{i}} t_{i}}\right)\right)^{1-\delta_{i}},
$$

As distribuições condicionais a posteriori dadas em (7.19) não possuem uma distribuição padrão, de modo que faremos uso do algoritmo de Metropolis-Hasting (veja, Chib e Greenberg, 1995) para gerar amostras a posteriori de $\alpha$ e $\beta$. 


\subsubsection{Estimador de Bayes do tempo médio de vida e para a proporção de sobreviventes dado um valor da covariável}

Considerando as amostras Gibbs geradas em (7.19) de $\alpha_{1}, \ldots, \alpha_{k_{1}}$ e $\beta_{1}, \ldots, \beta_{k_{2}}$, podemos obter uma estimativa de Monte Carlo para os momentos de interesse. Um caso especial deste é dado pelo tempo médio de vida dado em (7.2) para um valor especificado da covariável $\mathrm{x}_{h}$. Um estimador de Bayes de $\lambda_{h}$ com respeito à função de perda quadrática é dada por

$$
E\left[\lambda_{h} \mid D\right]=\int \ldots \int \exp \left\{\alpha_{1} x_{1 h}+\ldots+\alpha_{k_{1} h}\right\} \pi(\alpha, \beta \mid D) d \alpha d \beta
$$

a qual pode-se aproximar por sua estimativa de Monte Carlo

$$
\hat{\lambda}_{h}=\frac{2}{R S^{\prime}} \sum_{r=1}^{R} \sum_{s=\frac{S}{2}+1}^{S} \exp \left\{\alpha_{1}^{(r, s)} x_{1 h}+\ldots+\alpha_{k_{1} h}^{(r, s)}\right\},
$$

onde $\alpha_{1}^{(r, s)}, \ldots, \alpha_{k_{1}}^{(r, s)}$, denota os valores de $\alpha_{1}, \ldots, \alpha_{k_{1}}$ respectivamente, obtidos na $r$-ésima iteração e a $s$ - ésima replica, $R$ e $S$ são respectivamente, número total de iterações e simulações no amostrador de Gibbs e $\mathrm{x}_{h}$ é um valor da covariável.

Similarmente uma estimativa da proporção de sobreviventes em (7.3) para um valor da covariável $\mathrm{y}_{h}$ com respeito à função perda quadrática é dada por $E\left[p_{h} \mid D\right]$. Um estimador de Monte Carlo é dado por

$$
\hat{p}_{h}=\frac{2}{R S} \sum_{r=1}^{R} \sum_{s=\frac{S}{2}+1}^{S}=\frac{\exp \left\{\beta_{1}^{(r, s)} y_{1 h}+\ldots+\beta_{k_{2}}^{(r, s)} y_{k_{2} h}\right\}}{1+\exp \left\{\beta_{1}^{(r, s)} y_{1 h}+\ldots+\beta_{k_{2}}^{(r, s)} y_{k_{2} h}\right\}}
$$

\subsection{Aplicação}

Com a finalidade de ilustrar a metodologia apresentada nas seções 2 e 3, consideramos dois exemplos: o primeiro consiste de um conjunto de dados simulados e o segundo são os dados analizados por Kersey et al.(1980). 


\subsubsection{Exemplo 1-Dados simulados}

A tabela 7.1, apresenta uma amostra de $n=30$ observações de tempos de sobrevivência geradas do modelo de mistura exponencial com covariáveis. Considerou-se a variável $b_{i}$ que indica se o indivíduo esta em risco o não, que tem distribuição de Bernoulli $\left(1, p_{i}\right)$ com $p_{i}=\frac{\exp \left\{2+x_{i 1}\right\}}{1+\exp \left\{2+x_{i 1}\right\}}$. Para os indivíduos em risco, simulou-se os tempos de falha (morte), $t_{i}^{*}$ a partir da distribuição exponencial com média dada em (7.2): $\lambda_{i}=\exp \left\{2,5+0,2 x_{i 1}\right\}$ e para as covariáveis gerou-se considerando a ditribuição normal com média zero e variância 4 e para os tempos de censura, simulou-se uma variável aleatoria uniforme no intervalo $(0,2)$.

Tabela 7.1: Amostra gerada do modelo de mistura exponencial com covariáveis.

\begin{tabular}{|rlll|clll|}
\hline \hline$i$ & $t_{i}$ & $\delta_{i}$ & $x_{i 1}$ & $i$ & $t_{i}$ & $\delta_{i}$ & $x_{i 1}$ \\
\hline 1 & 0,06707 & 1 & $-1,60000$ & 16 & 0,01053 & 0 & 0,32277 \\
2 & 0,05666 & 1 & 2,06390 & 17 & 0,03374 & 1 & 0,22997 \\
3 & 0,07656 & 1 & $-0,13747$ & 18 & 0,02408 & 1 & 3,14980 \\
4 & 0,40201 & 0 & $-2,60500$ & 19 & 0,17374 & 1 & $-2,04450$ \\
5 & 0,03569 & 1 & 3,33550 & 20 & 0,02615 & 1 & 3,62700 \\
6 & 0,13400 & 0 & $-1,79780$ & 21 & 0,00416 & 1 & 1,41050 \\
7 & 0,05527 & 1 & 1,10310 & 22 & 0,01355 & 1 & 0,15793 \\
8 & 0,31485 & 1 & 0,90310 & 23 & 0,06404 & 1 & 3,79480 \\
9 & 0,04123 & 1 & 1,73770 & 24 & 0,06758 & 1 & 1,80100 \\
10 & 0,27833 & 0 & $-3,39280$ & 25 & 0,02492 & 1 & 3,33170 \\
11 & 0,02419 & 0 & 2,08630 & 26 & 0,02895 & 0 & 1,04340 \\
12 & 0,01238 & 0 & 0,83944 & 27 & 0,05138 & 1 & 1,12030 \\
13 & 0,13172 & 1 & 2,16810 & 28 & 0,41689 & 0 & 1,00790 \\
14 & 0,13965 & 1 & 0,96796 & 29 & 0,06251 & 1 & 1,07880 \\
15 & 0,03017 & 1 & 2,45200 & 30 & 0,25799 & 0 & $-2,74850$ \\
\hline
\end{tabular}

\section{Análise clássica}

Nesta subseção apresenta-se uma aplicação da métodologia discutida na seção 2 considarando os dados da tabela 7.1 . Na tabela 7.2 , apresenta-se as estimativas de máxima verossimilhança e os intervalos de confiança de $95 \%$ admitindo-se a normalidade assintótica 
dos estimadores de máxima verossimilhança de $\alpha_{0}, \alpha_{1}, \beta_{0}$ e $\beta_{1}$.

Tabela 7.2: Estimativas de máxima verossimilhança dos parâmetros do modelo de mixtura exponencial com covariáveis

\begin{tabular}{|cccc|}
\hline & EMV & D.P & Intervalo de confiança $95 \%$ \\
\hline$\hat{\alpha}_{0}$ & 2,1323 & 0,4042 & $(1,1340 ; 2,9246))$ \\
$\hat{\alpha}_{1}$ & 0,30143 & 0,1798 & $(-0,0510 ; 0,6538)$ \\
$\hat{\beta}_{0}$ & 2,0295 & 1,0657 & $(-0,0393 ; 4,1183)$ \\
$\hat{\beta}_{1}$ & 0,9581 & 0,5432 & $(-0,1065 ; 2,0227)$ \\
\hline
\end{tabular}

\section{Análise Bayesiana pelo método MCMC}

Nesta subseção desenvolve-se a análise Bayesiana apresentada na seção 3 para o modelo de sobrevivência com sobreviventes de longo período com covariáveis utilizando-se o algoritmo de Gibbs com Metropolis-Hasting (G-MH) para os dados da tabela 7.1.

Para a inferência Bayesiana consideramos as densidades a priori dada em (7.15) com $\alpha_{j} \sim N\left(0,10^{5}\right), j=0,1$ e $\beta_{k} \sim N\left(0,2 \times 10^{8}\right), k=0,1$. quase não informativas. Para se obter amostras de Gibbs através do algoritmo G-MH, procede-se da seguinte forma: geram-se 20 cadeias paralelas cada um com 4.000 iterações. Deprezam-se as 2.000 primeiras iterações, com objetivo de diminuir o efeito dos pontos iniciais, e a partir daí escolhem-se as iterações de 20 em 20, ou seja para cada parâmetro em cada cadeia considera-se a geração 1.020 - ésima, . ., 2.000 - ésima fornecendo uma amostra Gibbs de tamanho 2.000. Para monitorar a convergência utiliza-se a técnica de Gelman e Rubin (1992), método este que utiliza a técnica de análise de variância para verificar se mais iterações são necessárias. Na tabela 7.3, apresenta-se o resumo da distribuição a posteriori (7.18) e na figura 7.1 representa-se a densidade aproximada dos parâmetros do modelo de mistura exponencial com covariáveis. Também na tabela 7.3), tem-se o resultado do fator de redução de escala potencial estimada $\hat{R}$ (veja, Gelman e Rubin, 1992) para todos os parâmetros. O número 
de iterações considerado foi suficiente para se chegar à convergência, pois $\hat{R}<1.1$ para todos os parâmetros.

Tabela 7.3: Sumário a posteriori dos parâmetros do modelo de mistura exponencial com covariáveis

\begin{tabular}{|cccccc|}
\hline \hline & Medía & Mediana & D.P. & Intervalo de credibilidade $95 \%$ & $\hat{R}$ \\
\hline$\alpha_{0}$ & 2,3348 & 2,3388 & 0,2495 & $(1,8501 ; 2,8866)$ & 1,08677 \\
$\alpha_{1}$ & 0,1858 & 0,1849 & 0,1469 & $(-0,0969 ; 0,4772)$ & 1,00383 \\
$\beta_{0}$ & 2,0976 & 2,0476 & 0,7348 & $(0,7889 ; 3,6790)$ & 1,01045 \\
$\beta_{1}$ & 1,0536 & 1,0574 & 0,3782 & $(0,3109 ; 1,7908)$ & 1,00442 \\
\hline
\end{tabular}
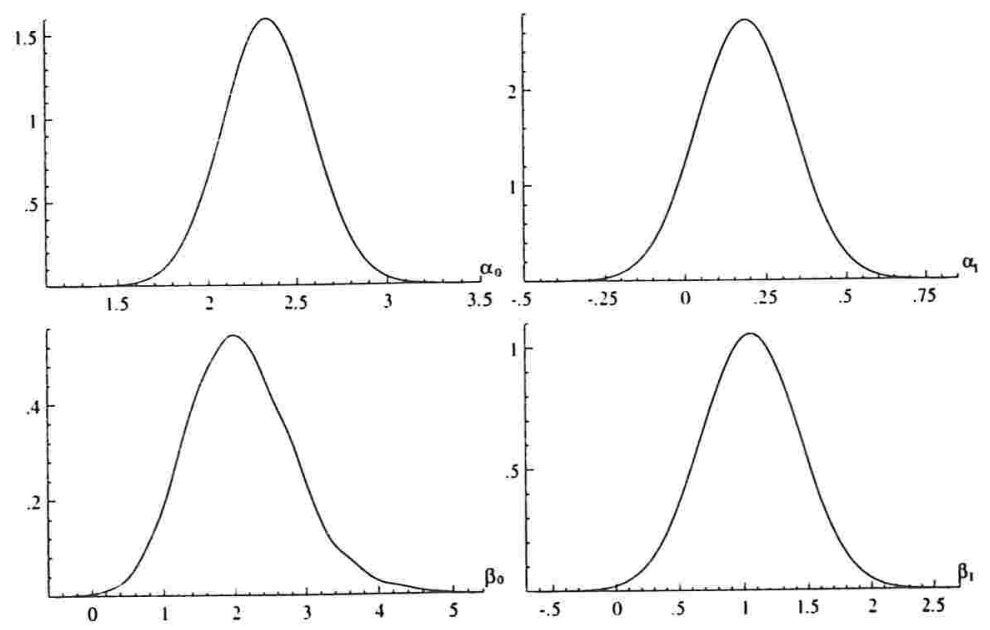

Figura 7.1: Densidades marginais a posteriori aproximadas dos parâmetros do modelo de mistura exponencial com covariáveis

Note que o desvio padrão do enfoque Bayesiano é bem menor que dos estimadores de máxima verossimilhança, mas, as médias a posteriori dos parâmetros do modelo são muito próximas aos estimadores de máxima verossimilhança. Contudo, é importante notar a redução na variância obtida com o procedimento Bayesiano, em alguns casos a redução 
chega a quase $50 \%$.

Na tabela 7.4, apresenta-se estimativas do tempo médio de vida e da proporção de sobreviventes dado um valor da covariável, $\mathrm{x}_{h}=\mathrm{y}_{h}=\left(\begin{array}{ll}1 & 0,2\end{array}\right)$.

Tabela 7.4: Estimativas do tempo médio e da proporção de sobreviventes dado $\mathrm{x}_{h}=$ $(10,2)$

\begin{tabular}{|llll|}
\hline \hline & Valor real & EMV & G-MH \\
\hline$\lambda_{h}$ & 12.679 & 8.958 & 11.033 \\
$p_{h}$ & 0.900 & 0.850 & 0.892 \\
\hline
\end{tabular}

Pode-se verificar através da tabela 7.4 resultados próximos para as estimativas de tempo de vida média e da proporção de sobreviventes obtidos pelo algoritmo de Gibbs com Metropolis-Hasting para a covariável $\mathrm{x}_{h}=\mathrm{y}_{h}=\left(\begin{array}{ll}1 & 0,2\end{array}\right)$. Na figura 7.2, representa-se as densidades a posteriori do tempo médio $\lambda_{h}$ e da proporção de sobreviventes, $p_{h}$ para um valor da covariável.

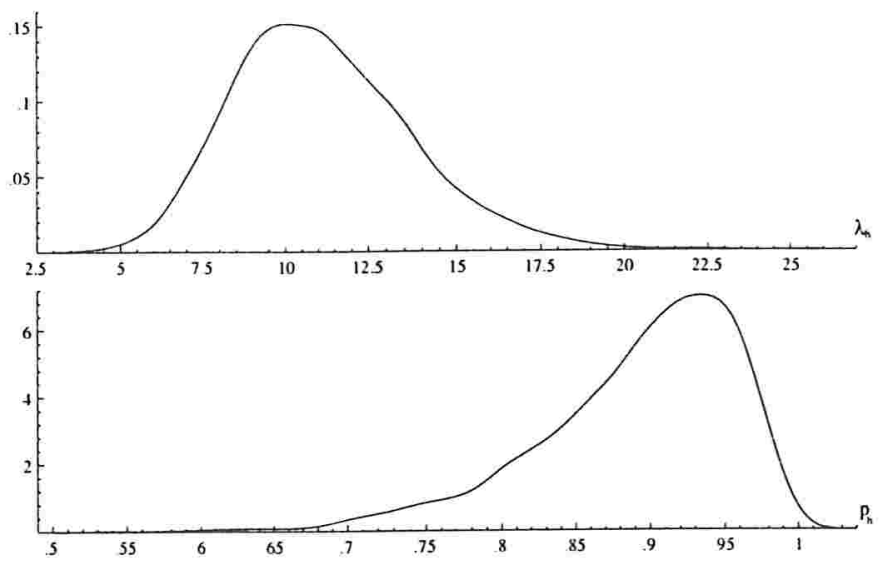

Figura 7.2: Densidades marginais a posteriori aproximados do tempo médio de vida e da proporção de sobreviventes para um valor dado da covariável $\mathrm{x}_{h}=\left(\begin{array}{ll}1 & 0,2\end{array}\right)$ 


\subsubsection{Exemplo 2-Dados de Kersey et al. 1987}

Nesta subseção apresentamos um exemplo como aplicação dos procedimentos clássicos e bayesianos, discutidos nas seções anteriores. O exemplo consiste em analisar os dados (veja tabela 6.6) obtidos por Kersey et al. (1987), os quais se referem aos tempos de recaída de pacientes de leucemia submetidos a dois tipos de transplante, "allogeneic" (Grupo 1) ou "autologous" (Grupo 2). Neste caso cosideraremos que o vetor de covariáveis $\mathbf{x}_{i}$ associado ao $i$-ésimo indivíduo tem a seguinte forma:

$$
\mathrm{x}_{i}=\left(1, x_{i}\right),
$$

onde $x_{i}=1$ para o Grupo 1 e $x_{i}=2$ para o Grupo 2.

\section{Análise clássica}

Nesta subseção apresenta-se uma aplicação da métodologia discutida na seção 2 considarando os dados da tabela 6.6. Considerando a log-verossimilhança dada em (7.16) temos que o estimador de máxima verossimilhança do parâmetro $\theta=\left(\alpha_{0}, \alpha_{1}, \beta_{0}, \beta_{1}\right)$ é obtido pela resolução do sistema de equações não lineares $S_{n}(\theta)=0$. Isto é feito através da subrotina de Newton-Rapson do pacote Ox.

As estimativas de máxima verossimilhança são dadas por $\hat{\alpha}_{0}=-0,2664(0,4213), \quad \hat{\alpha}_{1}=0.6263(0,2581), \quad \hat{\beta}_{0}=0,6146(0,7809)$, e $\hat{\beta}_{1}=0,3746(0,5081)$. Cada valor entre parênteses ao lado das estimativas representa a raiz quadrada do correspondente elemento da diagonal de $F_{n}^{-1}\left(\hat{\alpha}_{0}, \hat{\alpha}_{1}, \hat{\beta}_{0}, \hat{\beta}_{1}\right)$, onde $F_{n}\left(\alpha_{0}, \alpha_{1}, \beta_{0}, \beta_{1}\right)$ é a matriz de informação observada.

\section{Análise Bayesiana pelo método MCMC}

Nesta subseção desenvolve-se a análise bayesiama apresentada na seção 3 para o modelo de sobrevivência com sobreviventes de longo período com covariáveis utilizando-se o 
algoritmo de Gibbs com Metropolis-Hasting (G-MH) para os dados da tabela 6.6.

Para a inferência Bayesiana consideramos as densidades a priori dada em (7.15) com $\alpha_{j} \sim N\left(0,10^{5}\right), j=0,1$ e $\beta_{k} \sim N\left(0,2 \times 10^{8}\right), k=0,1$, quase não informativas. Para se obter uma amostra de Gibbs através do algoritmo G-MH, procede-se da seguinte forma: geram-se 20 cadeias paralelas cada um com 3.000 iterações. Deprezam-se as 1.500 primeras iterações, com objetivo de diminuir o efeito dos pontos iniciais, e a partir daí escolhem-se as iterações de 15 em 15, ou seja para cada parâmetro em cada cadeia considera-se as iterações 1.015-ésima, . . , 2.000-ésima que fornece uma amostra Gibbs de tamanho 1.500. Para monitorar a convergência utiliza-se a técnica de Gelman e Rubin (1992), método este que utiliza a técnica de análise de variância para verificar se mais iterações são necessárias. Na tabela 7.j, apresenta-se o resumo das distribuições a posteriori (7.18) e na figura 7.3 , representa-se as densidades aproximadas dos parâmetros do modelo de mistura exponencial com covariáveis. Também na tabela 7.5, tem-se o resultado do fator de redução de escala potencial estimada $\hat{R}$ (veja, Gelman e Rubin, 1992) para todos os parâmetros. O número de iterações considerado foi suficiente para se chegar à convergência, pois $\hat{R}<1.1$ para todos os parâmetros.

Tabela 7.5: Sumário a posteriori dos parâmetros do modelo de mistura exponencial com covariáveis

\begin{tabular}{|cccccc|}
\hline \hline & Medía & Mediana & D.P. & Intervalo de credibilidade $95 \%$ & $\hat{R}$ \\
\hline$\alpha_{0}$ & $-0,2578$ & $-0,2588$ & 0,0981 & $(-0,4381 ;-0,0782)$ & 1,001057 \\
$\alpha_{1}$ & 0,6011 & 0,6017 & 0,0875 & $(0,4301 ; 0,7830)$ & 1,001083 \\
$\beta_{0}$ & 0,6170 & 0,6208 & 0,2682 & $(0,0967 ; 1,1374)$ & 1,010045 \\
$\beta_{1}$ & 0,3872 & 0,3868 & 0,1883 & $(0,0237 ; 0,7591)$ & 1,00026 \\
\hline
\end{tabular}

$\mathrm{Na}$ tabela 7.6, apresenta-se a estimativa do tempo médio de vida e a proporção de sobreviventes para os Grupos 1 e 2, e na figura 7.4 , representa-se suas respectivas densidades 

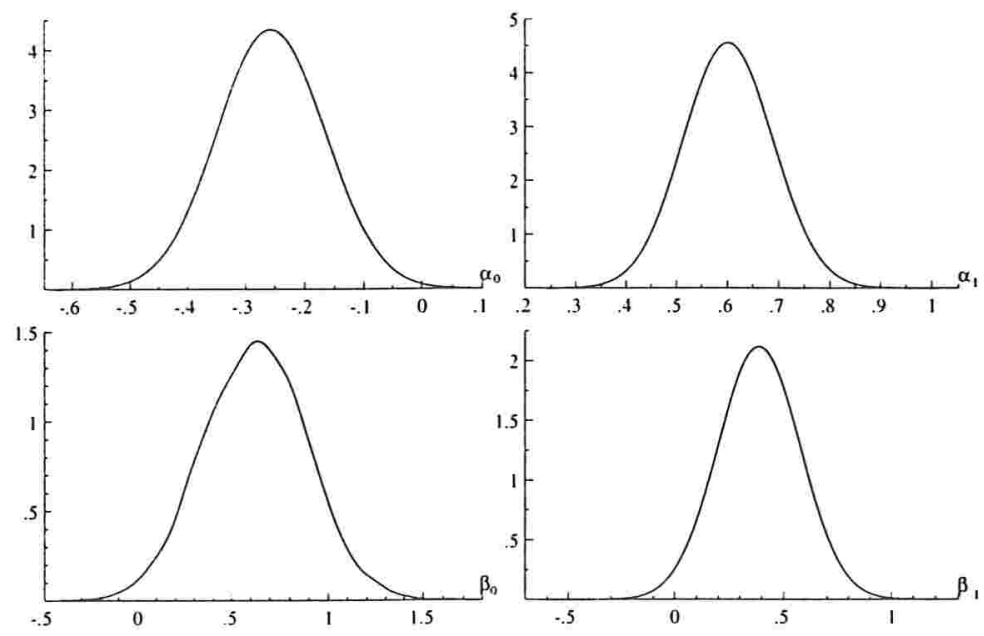

Figura 7.3: Densidades marginais a posteriori aproximadas dos parâmetros do modelo de mistura exponencial com covariáveis

a posteriori aproximadas.

Tabela 7.6: Estimativas do tempo médio e a proporção de sobreviventes para os Grupos 1 e 2

\begin{tabular}{|lllll|}
\hline \hline \multicolumn{2}{|c|}{ Grupo 1 } & \multicolumn{2}{c|}{ Grupo 2 } \\
\hline & MV & G-MH & MV & G-MH \\
\hline$\lambda$ & 1,4332 & 1,5678 & 2,6810 & 2,7945 \\
$p$ & 0,7289 & 0,7243 & 0,7964 & 0,7975 \\
\hline
\end{tabular}



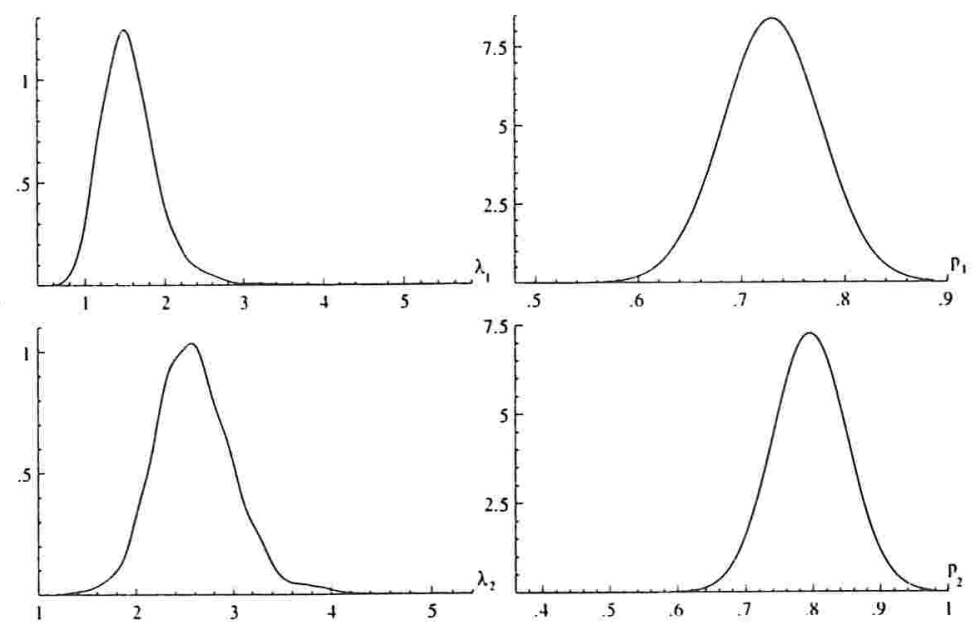

Figura 7.4: Densidades marginais a posteriori aproximadas do tempo médio de vida e a proporção de sobreviventes para os Grupos 1 e 2. 


\section{Capítulo 8}

\section{Modelo de mistura}

\section{Weibull-exponenciada}

\subsection{Introdução}

A distribuição Weibull-exponenciada (WE) foi introduzida por Mudholkar et al. (1995) e representa uma geralização das distribuiçōes Weibull e exponencial. Neste Cápitulo consideramos a análise de sobrevivência com indivíduos imunes na população considerando a distribuição WE para os indivíduos que falham (modelo de mistura WE) e estuda-se o uso deste modelo no teste de adequação dos modelos Weibull e exponencial desde a perspectiva clássica e Bayesiana.

\subsection{Modelo}

Seguindo a formulação de Farewell (1977), considera-se $b_{i}, i=1, \ldots, n$, uma variável binaria que indica $i$-ésimo indivíduo de uma população está em risco ou não com relação a algum tipo de falha $\left(b_{i}=1\right.$ se o indivíduo pertence ao grupo de risco e $b_{i}=0$ caso 
contrário). Considera-se que os $b_{i}$ são independentes e identicamente distribuidos tais que $P\left(b_{i}=1\right)=p \in(0,1)$ indica a proporção de indivíduos em risco e $P\left(b_{i}=0\right)=1-p$ a proporção de indivíduos imunes ou proporção de indivíduos curados de uma certa doença o itens que nunca falham.

As variáveis $b_{i}$ são não observadas mas, assume-se que dado que os indivíduos pertencem ao grupo de risco, isto é, $b_{i}=1, i=1, \ldots, n$, os tempos de falha $t_{i}^{*}$, são independentes e identicamentes distribuidos com distribuição Weibull-exponenciada. Isto é,

$$
g(t ; \alpha, \theta, \lambda \mid b=1)=\alpha \theta \lambda^{\alpha}\left[1-\exp \left\{-(\lambda t)^{\alpha}\right\}\right]^{\theta-1} \exp \left\{-(\lambda t)^{\alpha}\right\} t^{\alpha-1}
$$

onde $\alpha>0$ e $\theta>0$ são os parâmetros de forma e $\lambda>0$ o é parâmetro de escala. Observe que quando $\theta=1,(8.1)$ é função densidade da distribuição Weibull e quando $\alpha=1$ e $\theta=1,(8.1)$ é a densidade da distribuição exponencial.

Associado com o $i$-ésimo indivíduo está um tempo de censura $c_{i}$. A distribuição dos tempos de censura são considerados não informativos, isto é, não envolve $(\alpha, \theta, \lambda, p)$. Consideramos que $t_{i}^{*}$ e $c_{i}, i=1, \ldots, n$ são estocasticamente independentes. Para os indivíduos com $b_{i}=1$, observamos tempo de censura ou falha (morte), $t_{i}=\min \left(t_{i}^{*}, c_{i}\right)$ e o indicador de censura $\delta_{i}=1$ se ocorre falha e $\delta_{i}=0$, caso contrário. Quando $b_{i}=0$. observamos $t_{i}=c_{i}$ e, neste caso todos os tempos são censurados.

\subsection{Uma análise clássica do modelo de mistura Weibull- exponenciada}

Nesta seção tratamos de procedimentos inferênciais para o modelo de mistura Wibullexponenciada. Fornecemos a seguir procedimentos de estimação dos parâmetros pelo método de máxima verossimilhança, assim como métodos assintóticos para a construção de testes e intervalos de confiança aproximados para os parâmetros do modelo. 


\subsubsection{Estimação dos parâmetros por máxima verossimilhança}

Sejam $t_{1}, \ldots, t_{n}$ observações de uma amostra onde $t_{i}$ representa o tempo de vida ou o tempo de censura de cada um dos $n$ indivíduos em estudo. Seja $\boldsymbol{\eta}=(\alpha, \theta, \lambda, p)^{\prime} \in\{\boldsymbol{\eta}$ : $\alpha>0, \theta>0, \sigma>0,0<p<1\}$ o vetor de parâmetros a ser estimado.

Um indivíduo que falhou no tempo $t_{i}$, contribui para a função de verossimilhança com a parcela

$$
P\left\{b_{i}=1\right\} g\left(t ; \alpha, \theta, \sigma \mid b_{i}=1\right)=p \alpha \theta \lambda^{\alpha}\left[1-\exp \left\{-\left(\lambda t_{i}\right)^{\alpha}\right\}\right]^{\theta-1} \exp \left\{-\left(\lambda t_{i}\right)^{\alpha}\right\} t_{i}^{\alpha-1},
$$

enquanto, que um indivíduo que não falhou até o tempo $t_{i}$ contribui para a função de verossimilhança com a parcela

$$
P\left\{b_{i}=0\right\}+P\left\{b_{i}=1\right\} P\left(t_{i}^{*}>t_{i} ; \alpha, \theta, \sigma \mid b_{i}=1\right)=1-p\left[1-\exp \left\{-\left(\lambda t_{i}\right)^{\alpha}\right\}\right]^{\theta},
$$

que é a probabilidade do indivíduo ser um sobrevivente por longo periódo mais a probabilidade de um indivíduo sobreviver pelo menos até o tempo $t_{i}$. Portanto, a contribuição do $i$-ésimo indivíduo para a função de verosśimilhança é dada por

$$
\left(p \alpha \theta \lambda^{\alpha}\left[1-\exp \left\{-\left(\lambda t_{i}\right)^{\alpha}\right\}\right]^{\theta-1} \exp \left\{-\left(\lambda t_{i}\right)^{\alpha}\right\} t_{i}^{\alpha-1}\right)^{\delta_{i}}\left(1-p\left[1-\exp \left\{-\left(\lambda t_{i}\right)^{\alpha}\right\}\right]^{\theta}\right)^{1-\delta_{i}} \text {. }
$$

Logo, dada uma amostra $t_{1}, \ldots, t_{n}, \delta_{1}, \ldots, \delta_{n}$, a função log-verossimilhança para $\eta$ é dada por

$$
\begin{aligned}
\ell(\alpha, \theta, \lambda, p) & =r(\log (p \alpha \theta)+\alpha \log \lambda)-\lambda^{\alpha} \sum_{i=1}^{n} \delta_{i} t_{i}^{\alpha}+(\theta-1) \sum_{i=1}^{n} \delta_{i} \log \left(1-\exp \left(-\left(\lambda t_{i}\right)^{\alpha}\right)\right) \\
& +(\alpha-1) \sum_{i=1}^{n} \delta_{i} \log t_{i}+\sum_{i=1}^{n}\left(1-\delta_{i}\right) \log \left(1-p\left(1-\exp \left(-\left(\lambda t_{i}\right)^{\alpha}\right)\right)^{\theta}\right)
\end{aligned}
$$

O estimador de máxima verossimilhança de $\eta$ é obtido maximizando (8.2), o que equivale a resolver o sistema de equações $S(\boldsymbol{\eta})=\sum_{i=1}^{n} s_{i}(\boldsymbol{\eta})=0, \operatorname{com} s_{i}(\boldsymbol{\eta})=\left(s_{i 1}(\boldsymbol{\eta}), s_{i 2}(\boldsymbol{\eta}), s_{i 3}(\boldsymbol{\eta}), s_{i 4}(\boldsymbol{\eta})\right)^{\prime}$, onde

$s_{i 1}(\boldsymbol{\eta})=\frac{\partial \ell_{i}}{\partial \alpha}=\delta_{i}\left(\alpha^{-1}+\log (\lambda)+\frac{(\theta-1)\left(\lambda t_{i}\right)^{\alpha} \log \left(\lambda t_{i}\right) e^{-\left(\lambda t_{i}\right)^{\alpha}}}{1-e^{-\left(\lambda t_{i}\right)^{\alpha}}}-\left(\lambda t_{i}\right)^{\alpha} \log \left(\lambda t_{i}\right)+\log \left(t_{i}\right)\right)-$ 


$$
\begin{aligned}
& \frac{\left(1-\delta_{i}\right) p\left(1-e^{-\left(\lambda t_{i}\right)^{\alpha}}\right)^{\theta} \theta\left(\lambda t_{i}\right)^{\alpha} \log \left(\lambda t_{i}\right) e^{-\left(\lambda t_{i}\right)^{\alpha}}}{\left(1-e^{-(\lambda t)^{\alpha}}\right)\left(1-p\left(1-e^{-\left(\lambda t_{i}\right)^{\alpha}}\right)^{\theta}\right)} \\
& s_{i 2}(\boldsymbol{\eta})=\frac{\partial \ell_{i}}{\partial \theta}=\delta_{i}\left(\theta^{-1}+\log \left(1-e^{-\left(\lambda t_{i}\right)^{\alpha}}\right)\right)-\frac{\left(1-\delta_{i}\right) p\left(1-e^{-\left(\lambda t_{i}\right)^{\alpha}}\right)^{\theta} \log \left(1-e^{-\left(\lambda t_{i}\right)^{\alpha}}\right)}{1-p\left(1-e^{-\left(\lambda t_{i}\right)^{\alpha}}\right)^{\theta}} \\
& s_{i 3}(\boldsymbol{\eta})=\frac{\partial \ell_{i}}{\partial \lambda}=\delta_{i}\left(\frac{\alpha}{\lambda}+\frac{(\theta-1)\left(\lambda t_{i}\right)^{\alpha} \alpha e^{-\left(\lambda t_{i}\right)^{\alpha}}}{\lambda\left(1-e^{-\left(\lambda t_{i}\right)^{\alpha}}\right)}-\frac{\left(\lambda t_{i}\right)^{\alpha} \alpha}{\lambda}\right)- \\
& \frac{\left(1-\delta_{i}\right) p\left(1-e^{-\left(\lambda t_{i}\right)^{\alpha}}\right)^{\theta} \theta\left(\lambda t_{i}\right)^{\alpha} \alpha e^{-\left(\lambda t_{i}\right)^{\alpha}}}{\lambda\left(1-e^{-\left(\lambda t_{i}\right)^{\alpha}}\right)\left(1-p\left(1-e^{-\left(\lambda t_{i}\right)^{\alpha}}\right)^{\theta}\right)} \text {, } \\
& s_{i 4}(\boldsymbol{\eta})=\frac{\partial \ell_{i}}{\partial p}=\frac{\delta_{i}}{p}-\frac{\left(1-\delta_{i}\right)\left(1-e^{-\left(\lambda t_{i}\right)^{\alpha}}\right)^{\theta}}{1-p\left(1-e^{-\left(\lambda t_{i}\right)^{\alpha}}\right)^{\theta}}
\end{aligned}
$$

sendo $\ell_{i}$ a $i$-ésima componente da função log-verossimilhança. Do sistema de equaçôes não lineares dado acima não consiguimos obter analiticamente os estimadores de máxima verossimilhança, e precisamos de um método iterativo para solucionar o sistema. Este problema pode ser resolvido usando método de Newton-Raphson o qual aparece como rotina em alguns pacotes tais como Ox (veja, Doornik, 1998).

\subsubsection{Inferências sobre os parâmetros $\alpha, \theta, \lambda$ e $p$}

Para fazermos inferências sobre os parâmetros $\alpha, \theta, \lambda$ e $p$, utilizaremos a normalidade assintótica dos estimadores de máxima verosssimilhança (EMV). Assim, para $\hat{\alpha}, \hat{\theta}, \hat{\lambda}$ e $\hat{p}$ EMV's de $\alpha, \theta, \lambda$ e $p$, ponto interior do espaço paramétrico e sob certas condições de regularidade (veja, Cox e Hinkley, 1974) pode-se mostrar, para $n \longrightarrow \infty$, que

$$
i^{1 / 2}(\alpha, \theta, \lambda, p)\left[\left(\begin{array}{c}
\hat{\alpha} \\
\hat{\theta} \\
\hat{\lambda} \\
\hat{p}
\end{array}\right)-\left(\begin{array}{c}
\alpha \\
\theta \\
\lambda \\
p
\end{array}\right)\right] \stackrel{D}{\longrightarrow} N\left(0, I_{4}\right)
$$

onde $i^{1 / 2}(\alpha, \theta, \lambda, p)$ é a matriz raiz quadrada da matriz de informação de Fisher, $N\left(0, I_{4}\right)$ denota a distribuição normal multivariada com vetor de médias igual 0 e matriz de 
variâncias $I_{4}\left(I_{4}\right.$ denota a matriz indentidade de ordem $\left.4 \times 4\right)$. A normalidade assintótica (8.7) segue sendo válida se $i(\alpha, \theta, \lambda, p)$ é substituido por seu estimador consistente $i(\hat{\alpha}, \hat{\theta}, \hat{\lambda}, \hat{p})$ ou simplesmente considerarmos a matriz de informação avaliada nos estimadores de máxima verossimilhança denotada por $I(\hat{\alpha}, \hat{\theta}, \hat{\lambda}, \hat{p})$.

Considerando a distribuição assintótica normal dada em (8.7) para os estimadores de máxima verossimilhança $\hat{\alpha}, \hat{\theta}, \hat{\lambda}$ e $\hat{p}$, podemos construir intervalos para $\alpha, \theta, \lambda$ e $p$, considerando um nivel de confiança $100(1-\gamma) \%$, dados por,

$$
\begin{aligned}
i c(\alpha) & =\left(\hat{\alpha}-z_{\gamma / 2}\left(I^{(11)}\right)^{1 / 2} ; \hat{\alpha}+z_{\gamma / 2}\left(I^{(11)}\right)^{1 / 2}\right), \\
i c(\theta) & =\left(\hat{\theta}-z_{\gamma / 2}\left(I^{(22)}\right)^{1 / 2} ; \hat{\theta}+z_{\gamma / 2}\left(I^{(33)}\right)^{1 / 2}\right), \\
i c(\lambda) & =\left(\hat{\lambda}-z_{\gamma / 2}\left(I^{(33)}\right)^{1 / 2} ; \hat{\lambda}+z_{\gamma / 2}\left(I^{(33)}\right)^{1 / 2}\right), \\
i c(p) & =\left(\hat{p}-z_{\gamma / 2}\left(I^{(44)}\right)^{1 / 2} ; \hat{p}+z_{\gamma / 2}\left(I^{(44)}\right)^{1 / 2}\right),
\end{aligned}
$$

onde $I^{(j j)}, j=1,2,3,4$, é o $j$-ésimo elemento diagonal da matriz $i^{-1}(\hat{\alpha}, \hat{\theta}, \hat{\lambda}, p)$ e $z_{\gamma / 2}$ é um percentil da distribuição normal padrão.

\subsubsection{Teste do modelo de mistura Weibull}

Considerando as hipóteses

$$
H_{0}: \theta=1 \text { versus } H_{1}: \theta \neq 1 \text {, }
$$

estaremos testando a adequação do modelo de mistura Weibull para os tempos de vida. Usamos especificamente a estatística da razão de verossimilhança

$$
\Lambda=-2\{\ell(\tilde{\alpha}, 1, \tilde{\lambda}, \tilde{p})-\ell(\hat{\alpha}, \hat{\theta}, \hat{\lambda}, \hat{p})\}
$$

onde $\ell(\tilde{\alpha}, 1, \tilde{\lambda})$ corresponde ao máximo de $\ell(\alpha, 1, \lambda)$ dado por $\ell(\alpha, 1, \lambda, p)=r(\log (p \alpha)+\alpha \log \lambda)-\lambda^{\alpha} \sum_{i=1}^{n} \delta_{i} t_{i}^{\alpha}+(\alpha-1) \sum_{i=1}^{n} \delta_{i} \log t_{i}+\sum_{i=1}^{n}\left(1-\delta_{i}\right) \log \left(1-p+p \exp \left(-\left(\lambda t_{i}\right)^{\alpha}\right)\right.$ A estatística (8.13) para grandes amostras, tem distribuição aproximada qui-quadrado com um grau de liberdade sob a hipóteses $H_{0}$. 


\subsubsection{Teste para o modelo de mistura exponencial}

Considerar

$$
H_{0}:\left(\begin{array}{l}
\theta \\
\alpha
\end{array}\right)=\left(\begin{array}{l}
1 \\
1
\end{array}\right) \text { versus, } H_{1}:\left(\begin{array}{l}
\theta \\
\alpha
\end{array}\right) \neq\left(\begin{array}{l}
1 \\
1
\end{array}\right)
$$

equivale a testar que os dados admitem um modelo de mistura exponencial para os tempos de vida e a estatística da razão de verossimilhanca usada para o teste é

$$
\Lambda=-2(\ell(1,1, \tilde{\lambda}, \tilde{p})-\ell(\hat{\alpha}, \hat{\theta}, \hat{\lambda}, \hat{p}))
$$

onde $\grave{\lambda}$ é o valor que maximiza a log-verossimilhança

$$
\ell(1,1, \lambda, p)=r \log (p)+r \log \lambda-\lambda \sum_{i=1}^{n} \delta_{i} t_{i}+\sum_{i=1}^{n}\left(1-\delta_{i}\right) \log \left(1-p+p \exp \left(-\lambda t_{i}\right)\right) .
$$

A estatística da razão de verossimilhança $(8.15)$, sob $H_{0}$ tem distribuição assintótica quiquadrado com 2 graus de liberdade.

\subsection{Uma análise Bayesiana para o modelo de mistura Weibull-exponenciada}

$\mathrm{Na}$ seção anterior foi considerada uma análise clássica para o modelo de mistura Weibullexponenciada baseada em resultados assintóticos que são válidos para amostras de tamanho grande. Já verificamos isto no caso exponencial. Para amostras de tamanhos moderados ou pequenos esses resultados assintóticos podem não ser confiáveis. Um procedimento alternativo que pode contornar esse problema é através da utilização de métodos Bayesianos que consideram os parâmetros como variáveis aleatórias.

Nesta seção, apresenta-se uma análise considerando-se a abordagem Bayesiana para o modelo de mistura Weibull-exponenciada. Neste ponto surgem duas dificuldades: a primeira se refere a obtenção da distribuição a posterior marginal e a segunda se refere ao cálculo de momentos de interesse. Em ambos casos é necessário a resolução de integrais que muitas vezes não apresentam solução análitica. Neste caso métodos de aproximação 
ou métodos numéricos são necessários. Um método muito utilizado é o método de approximação de Laplace (Tierney, Kass e Kadane, 1986). Para aplicação desse método é necessário máximizar funções que dependem de parâmetros, de modo que, a medida que a dimensão do espaço paramétrico aumenta, aumentam as dificuldades para sua obtenção e a aplicação do método vai se tornando mais trabalhosa e de difícil implementação. Para contornar esse problema neste trabalho utiliza-se os métodos de simulação de Monte Carlo via Cadeias de Markov, tais como o amostrador de Gibbs (Casela e George, 1992) e Metropolis-Hasting (Chib e Greenberg, 1995).

\subsubsection{Densidade a priori independentes}

Para representar o grau de conhecimento sobre os parâmetros do modelo de mistura Weibull-exponenciada, consideramos as seguintes densidades à priori para $\alpha, \theta, \lambda$ e $p$ :

$$
\begin{aligned}
\alpha & \sim \Gamma\left(\alpha_{0}, \alpha_{1}\right), \\
\theta & \sim \Gamma\left(\theta_{0}, \theta_{1}\right), \\
\lambda & \sim \Gamma\left(\lambda_{0}, \lambda_{1}\right), \\
p & \sim \operatorname{Beta}\left(\gamma_{0}, \gamma_{1}\right),
\end{aligned}
$$

onde $\Gamma(a, b)$ denota a distribuição Gama com média $a / b$, Beta $\left(\gamma_{0}, \gamma_{0}\right)$ denota a distribuição Beta com média $\frac{\gamma_{0}}{\left(\gamma_{0}+\gamma_{1}\right)}$, e $\alpha_{i}, \theta_{i}, \lambda_{i}$ e $\gamma_{i}, i=0,1$, são constantes conhecidas baseadas na informação a priori do especialista e da análise preliminar dos dados. Assumindo independência entre os parâmetros a densidade a priori conjunta é dada por.

$$
\pi(\alpha, \theta, \lambda, p) \propto p^{\gamma_{0}-1}(1-p)^{\gamma_{1}-1} \alpha^{\alpha_{0}-1} \theta^{\theta_{0}-1} \lambda^{\lambda_{0}} \exp \left\{-\alpha_{1} \alpha-\theta_{1} \theta-\lambda_{1} \lambda\right\}
$$

onde $\alpha>0 \theta>0, \lambda>0$ e $0<p<1$.

De (8.2) pode-se mostrar que a função verossimilhança para $\alpha, \theta, \lambda$ e $p$ é dada por $L(\alpha, \theta, \lambda, p)=\exp \{\ell(\alpha, \theta, \lambda, p)\}$, ísto é,

$L(\alpha, \theta, \lambda, p)=p^{r} \alpha^{r} \theta^{r} \lambda^{r \alpha} \exp \left\{-\lambda^{\alpha} \sum_{i=1}^{n} \delta_{i} t_{i}^{\alpha}+(\alpha-1) \sum_{i=1}^{n} \delta_{i} \log t_{i}+\right.$ 


$$
\left.(\theta-1) \sum_{i=1}^{n} \delta_{i} \log \left[1-\exp \left\{-\left(\lambda t_{i}\right)^{\alpha}\right\}\right]+\sum_{i=1}^{n}\left(\delta_{i}-1\right) \log \left(1-p\left[1-\exp \left\{-\left(\lambda t_{i}\right)^{\alpha}\right\}\right]^{\theta}\right)\right\}
$$

onde $r=\sum_{i=1}^{n} \delta_{i}$

\subsubsection{Densidade conjunta a posteriori para os parâmetros do modelo de mistura Weibull-exponenciada}

Considerando as densidade a priori (8.17) e a função de verossimilhança (\$.18), a densidade a posteriori conjunta para $\alpha, \theta, \lambda$ e $p$ é dada por (veja, por exemplo, Box e Tiao, 1973)

$$
\pi(\alpha, \theta, \lambda, p \mid D) \propto \pi(\alpha, \theta, \lambda, p) L(\alpha, \theta, \lambda, p)
$$

isto é,

$$
\begin{aligned}
\pi(\alpha, \theta, \lambda, p \mid D) \propto & p^{\gamma_{0}+r-1}(1-p)^{\gamma_{1}-1} \alpha^{\alpha_{0}+r-1} \theta^{\theta_{0}+r-1} \lambda^{\lambda_{0}+r \alpha-1} \exp \left\{-\lambda^{\alpha} \sum_{i=1}^{n} \delta_{i} t_{i}^{\alpha}-\alpha_{1} \alpha-\right. \\
& \theta_{1} \theta-\lambda_{1} \lambda+(\alpha-1) \sum_{i=1}^{n} \delta_{i} \log t_{i}+(\theta-1) \sum_{i=1}^{n} \delta_{i} \log \left[1-\exp \left\{-\left(\lambda t_{i}\right)^{\alpha}\right\}\right]+ \\
& \left.\sum_{i=1}^{n}\left(\delta_{i}-1\right) \log \left(1-p\left[1-\exp \left\{-\left(\lambda t_{i}\right)^{\alpha}\right\}\right]^{\theta}\right)\right\}
\end{aligned}
$$

onde $D$ denota o conjunto dos dados observados.

No enfoque bayesiano, inferências são baseadas nas densidades a posteriori marginais dos parâmetros envolvidos no modelo. Pode-se observar que densidade a posteriori conjunta (8.19) não é uma densidade padrão, portanto, só podemos avaliar as densidades marginais por meio de métodos de aproximação tais como o método de Laplace ou através de métodos de simulação de Monte Carlo via cadeia de Markov (MCMC). Nesta seção optamos pelo método de simulação por serem de fácil implementação computacional. Para obtermos uma amostra das densidades a posteriori marginais de $\alpha, \theta, \lambda$ e $p$, utiliza-se - amostrador de Gibbs que é uma técnica para gerar variáveis aleatórias de uma distribuição marginal inderetamente, isto é, este método permite gerar variáveis aleatórias de uma distribuição marginal sem ter que calcular a forma da densidade. 
De (8.19) pode-se mostrar que as densidades condicionais marginais $\pi(\alpha \mid \theta, \lambda, p, D)$, $\pi(\theta \mid \alpha, \lambda, p, D), \pi(\lambda \mid \alpha, \theta, p, D)$ e $\pi(p \mid \alpha, \theta, \lambda, D)$ para o algoritmo de Gibbs são dadas por,

(i) $\pi(\alpha \mid \theta, \lambda, p, D) \propto \alpha^{\alpha_{0}+r-1} \lambda^{r \alpha} \exp \left\{-\lambda^{\alpha} \sum_{i=1}^{n} \delta_{i} t_{i}^{\alpha}-\alpha\left(\alpha_{1}-\sum_{i=1}^{n} \delta_{i} \log t_{i}\right)+\Psi(\alpha \mid \theta, \lambda, p)\right\}$,

(ii) $\pi(\theta \mid \alpha, \lambda, p, D) \propto \theta^{\theta_{0}+r-1} \exp \left\{\theta\left(\theta_{0}-\sum_{i=1}^{n} \delta_{i} \log \left[1-\exp \left\{-\left(\lambda t_{i}\right)^{\alpha}\right\}\right]\right)+\right.$

$$
\left.\sum_{i=1}^{n}\left(\delta_{i}-1\right) \log \left(1-p\left[1-\exp \left\{-\left(\lambda t_{i}\right)^{\alpha}\right\}\right]^{\theta}\right)\right\},
$$

(iii) $\pi(\lambda \mid \alpha, \theta, p, D) \propto \lambda^{\lambda_{0}+r \alpha-1} \exp \left\{-\lambda^{\alpha} \sum_{i=1}^{n} \delta_{i} t_{i}^{\alpha}-\lambda \lambda_{1}+\Psi(\alpha \mid \theta, \lambda, p)\right\}$,

(iv) $\pi(p \mid \alpha, \theta, \lambda, D) \propto p^{\gamma_{0}+r-1}(1-p)^{\gamma_{1}-1} \exp \left\{\sum_{i=1}^{n}\left(\delta_{i}-1\right) \log \left(1-p\left[1-\exp \left\{-\left(\lambda t_{i}\right)^{\alpha}\right\}\right]^{\theta}\right)\right\}$,

onde

$\Psi(\alpha \mid \theta, \lambda, p)=(\theta-1) \sum_{i=1}^{n} \delta_{i} \log \left[1-\exp \left\{-\left(\lambda t_{i}\right)^{\alpha}\right\}\right]+\sum_{i=1}^{n}\left(\delta_{i}-1\right) \log \left(1-p\left[1-\exp \left\{-\left(\lambda t_{i}\right)^{\alpha}\right\}\right]^{\theta}\right)$

Observe em (i)-(iv) que as densidades são não conhecidas, portanto, utilizaremos o amostrador de Gibbs com algoritmo de Metropolis-Hasting para gerar as amostras a posteriori de $\alpha, \theta, \lambda$ e $p$.

O procedimento para a aplicação do algoritmo de Metropolis-Hasting dentro do ciclo do amostrador de Gibbs é ilustrado brevemente para geração de $\alpha$ em (8.20), para isto denotamos por $f(\alpha)$ a densidade condicional (i) já que $\alpha>0$ define-se a seguinte transformação $\alpha^{\prime}=\log \alpha$ e consideramos o nucleo de transição $q\left(\alpha^{\prime}, X\right)$ que leva $\alpha$ a $X$; por exemplo, $X=\alpha^{\prime}+\sigma Z$, sendo $Z$ uma variável aleatória normal padrão e $\sigma^{2}$ tem a variância de $\alpha^{\prime}$ e computamos $f_{\alpha^{\prime}}\left(\alpha^{\prime}\right)=f(\alpha)\left|d \alpha / d \alpha^{\prime}\right|$, sendo a probabilidade de movimento da cadeia $p=\min \left\{1, \frac{f_{\alpha^{\prime}}(X)}{f_{\alpha^{\prime}}\left(\alpha^{\prime}\right)}\right\}$. Em cada passo do algoritmo de Gibbs transformamos $\alpha^{\prime}$; $\alpha=\exp \left(\alpha^{\prime}\right)$. Similar procedimento pode ser aplicado para gerar $\theta, \lambda$ e para $p$ o nucleo de transição $U(0,1)$ (distribuição uniforme no intervalo $(0,1)$ ). 


\subsubsection{Densidade conjunta a posteriori para os parâmetros do modelo de mistura Weibull}

Considere as seguintes densidades a priori para $\alpha, \lambda$ e $p$ parâmetros do modelo de mistura Weibull:

$$
\begin{aligned}
& \alpha \sim \Gamma\left(\alpha_{0}, \alpha_{1}\right), \text { com } \alpha_{0} \text { e } \alpha_{1} \text { conhecidos } \\
& \lambda \sim \Gamma\left(\lambda_{0}, \lambda_{1}\right), \text { com } \lambda_{0} \text { e } \lambda_{1} \text { conhecidos } \\
& p \sim \operatorname{Beta}\left(\gamma_{0}, \gamma_{1}\right), \text { com } \gamma_{0} \text { e } \gamma_{1} \text { conhecidos. }
\end{aligned}
$$

Assumindo independência entre $\alpha, \lambda$ e $p$ e considerando a função de verossimilhança com $\theta=1$ em (8.18), pode-se mostrar que a densidade conjunta a posteriori de $\alpha, \lambda$ e $p$ é dada por

$$
\begin{gathered}
\pi(\alpha, \lambda, p \mid D) \propto p^{r+\gamma_{0}-1}(1-p)^{\gamma_{1}-1} \alpha^{r+\alpha_{0}-1} \lambda^{r \alpha+\lambda_{0}-1} \exp \left\{-\lambda^{\alpha} \sum_{i=1}^{n} \delta_{i} t_{i}-\alpha \alpha_{1}-\lambda \lambda_{1}\right\} \times \\
\prod_{i=1}^{n}\left(t_{i}^{\alpha-1}\right)^{\delta_{i}}\left(1-p+p \exp \left\{-\left(\lambda t_{i}\right)^{\alpha}\right\}\right)^{\delta_{i}-1},
\end{gathered}
$$

onde $D$ é o conjunto dos dados observados.

De (8.22) pode-se mostrar que as densidades condicionais marginais $\pi(\alpha \mid \lambda, p, D)$, $\pi(\lambda \mid \alpha, p, D)$ e $\pi(p \mid \alpha, \lambda, D)$ para o algoritmo de Gibbs são dadas por,

(i) $\pi(\alpha \mid \lambda, p, D) \propto \alpha^{r+\alpha_{0}-1} \lambda^{r \alpha} \exp \left\{-\lambda^{\alpha} \sum_{i=1}^{n} \delta_{i} t_{i}^{\alpha}-\alpha\left(\alpha_{1}+\sum_{i=1}^{n} \delta_{i} \log t_{i}\right)\right\} \Psi_{1}(\alpha, \lambda, p)$,

(ii) $\pi(\lambda \mid \alpha, p, D) \propto \lambda^{r \alpha+\lambda_{0}-1} \exp \left\{-\lambda^{\alpha} \sum_{i=1}^{n} \delta_{i} t_{i}^{\alpha}-\lambda \lambda_{1}\right\} \Psi_{1}(\alpha, \lambda, p)$,

(iii) $\pi(p \mid \alpha, \lambda, D) \propto p^{r+\lambda_{0}-1}(1-p)^{\lambda_{1}-1} \Psi_{1}(\alpha, \lambda, p)$,

onde $\Psi_{1}(\alpha, \lambda, p)=\prod_{i=1}^{n}\left(1-p+p \exp \left\{-\left(\lambda t_{i}\right)^{\alpha}\right\}\right)^{\delta_{i}-1}$.

Observe que as densidades a posteriori condicionais em (8.23) não possuem uma forma padrão, pelo que faremos uso do algoritmo de Metropolis-Hasting para gerar amostras a posteriori. 


\subsubsection{Teste de hipóteses Bayesiano}

Na seção anterior considerou-se o modelo de mistura Weibull-exponenciada para verificar se certo conjunto de dados de tempos de vida podem ser ajustados pelo modelo de mistura Weibull ou ou pelo modelo de mistura exponencial. Nesta seção testa-se as hipóteses dadas em (8.12) e (8.14) a partir de uma perpespectiva Bayesiana.

Como descrito no capítulo 1, consideramos a técnica das densidade preditivas condicionais ordenadas (CPO) definidas por

$$
f\left(t_{i} \mid \underline{t}_{(i)}\right)=\int f\left(t_{i} \mid \theta, \underline{t}_{(i)}\right) \pi\left(\theta \mid \underline{t}_{(i)}\right) d \theta
$$

onde $t_{(i)}=\left(t_{1}, \ldots, t_{i-1}, t_{i+1}, \ldots, t_{n}\right)$, para determinar a verossimilhança preditiva dos modelos sob as hipóteses nula e alternativa para testar a adequabilidade do modelo de mistura Weibull e do modelo de mistura exponencial, utilizando o pseudo-fator de Bayes (veja, por exemplo, Gelfand e Dey, 1994).

Usando as amostras geradas pelo algoritmo de Gibbs pode-se estimar a densidade preditiva (\$.24) para o modelo de mistura Weibull (modelo sob $H_{0}$ dado em (8.12)), que denotaremos por, $f\left(t_{i} \mid \underline{t}_{(i)}, M_{0}\right)$.A estimativa de Monte Carlo desta densidade é dada por,

$$
\hat{f}\left(t_{i} \mid \underline{t}_{(i)}, M_{0}\right)= \begin{cases}B\left(\sum_{k=1}^{B}\left[\alpha^{(k)}\left(\lambda^{(k)}\right)^{\alpha^{(k)}} p^{(k)} \exp \left\{-\left(\lambda^{(k)}\right)^{\alpha^{(k)}} t_{i}^{\alpha^{(k)}}\right\} t_{i}^{\alpha^{(k)}-1}\right]^{-1}\right)^{-1}, & \text { se } \delta_{i}=1 \\ B\left(\sum_{k=1}^{B}\left[1-p^{(k)}\left(1+\exp \left(-\left(\lambda^{(k)} t_{i}\right)^{\alpha^{(k)}}\right)\right)\right]^{-1}\right)^{-1}, & \text { se } \delta_{i}=0\end{cases}
$$

onde $B$ é o tamanho da amostra Gibbs, e $\left(\alpha^{(k)}, \lambda^{(k)}, p^{(k)}\right)$ é a $k$-ésima amostra de Gibbs de $\alpha, \lambda$ e $p$. A estimativa da verossimilhança preditiva sob $H_{0}(8.12)$ é dada por $\hat{f}\left(\underline{t} \mid M_{0}\right)=$ $\prod_{i=1}^{n} \hat{f}\left(t_{i} \mid \underline{t}_{(i)}, M_{0}\right)$.

Para aproximar a densidade preditiva para o modelo de mistura Weibull-exponenciada (modelo sob $H_{1}$ em (8.12)), que denotaremos por $\hat{f}\left(t_{i} \mid \underline{t}_{(i)}, M_{1}\right)$, uma estimativa de Monte 
Carlo com as amotras Gibbs dos parâmetros do modelo é dada por

$$
\hat{f}\left(t_{i} \mid \underline{t}_{(i)}, M_{1}\right)= \begin{cases}B\left(\sum _ { k = 1 } ^ { B } \left[\alpha^{(k)} \theta^{(k)}\left(\lambda^{(k)}\right)^{\alpha^{(k)}} p^{(k)} \exp \left\{-\left(\lambda^{(k)}\right)^{\alpha^{(k)}} t_{i}^{\alpha^{(k)}}\right\} t_{i}^{\alpha^{(k)}-1} \times\right.\right. & \\ \left.\left.\left(1-\exp \left\{-\left(\lambda^{(k)} t_{i}\right)^{\alpha^{(k)}}\right\}\right)^{\theta^{(k)}}\right]^{-1}\right)^{-1}, & \text { se } \delta_{i}=1 \\ B\left(\sum_{k=1}^{B}\left[1-p^{(k)}\left(1+\exp \left(-\left(\lambda^{(k)} t_{i}\right)^{\alpha^{(k)}}\right)\right)\right]^{-1}\right)^{-1}, & \text { se } \delta_{i}=0,\end{cases}
$$

onde $\left(\alpha^{(k)}, \theta^{(k)}, \lambda^{(k)}, p^{(k)}\right)$ é a $k$-ésima amostra Gibbs de $\alpha, \theta, \lambda$ e $p$. A estimativa da verossimilhança preditiva sob $H_{1}$ em (8.12) é dada por $\hat{f}\left(\underline{t} \mid M_{1}\right)=\prod_{i=1}^{n} \hat{f}\left(t_{i} \mid \underline{t}_{(i)}, M_{1}\right)$. Com (8.25) e (8.26) pode-se calcular o pseudo-fator de Bayes do modelo $M_{0}$ (modelo de mistura Weibull) com respeito ao modelo $M_{1}$ (modelo de mistura Weibull-exponenciada), que denotaremos por $P S F B_{01}$ e rejeita-se $H_{0}$ se $P S F B_{01}<1$.

\subsection{Exemplos de aplicação}

Nesta seção apresentamos dois exemplos como aplicação dos procedimentos clásicos e Bayesianos, discutidos nas seções anteriores.

\subsubsection{Exemplo 1-Dados Simulados}

Na tabela 8.1 , apresenta-se uma amostra de $n=30$ observações de tempos de sobrevivência geradas do modelo de mistura Weibull-exponenciada. Considerou-se que a variável $b_{i}$ que indica se o indivíduo esta em risco o não, tem distribuição Bernoulli $(1, p)$ com $p=0.7$. Para os indivíduos em riscos, simulou-se os tempos de falha (morte), $t_{i}^{*}$ tendo distribuição Weibull-exponenciada com parâmetros $\alpha=4, \theta=1$ e $\lambda=3$ e para os tempos de censura simulou-se uma variável aleatória exponencial com média 6 . 
Tabela 8.1: Dados gerados do modelo de mistura Weibull-exponenciada com + indicando censura

\begin{tabular}{llllllllll}
\hline \hline 0,23242 & 0,073788 & $0,50877^{+}$ & 0,27088 & $6,3350^{+}$ & 0,27678 & $0,045127^{+}$ & $8,2059^{+}$ & $3,0524^{+}$ & 0,45935 \\
0,29664 & 0,37975 & 0,14298 & $3,5607^{+}$ & 0,28250 & 0,41267 & 0,29316 & 0,27130 & 0,32717 & 0,19019 \\
$2,6033^{+}$ & 0,33151 & 0,30793 & 0,33978 & 0,19775 & $0,38698^{+}$ & $1,6313^{+}$ & $0,25243^{+}$ & $10,769^{+}$ & 0,25877 \\
\hline \hline
\end{tabular}

\section{Análise clássica}

Considerando a log-verossimilhança dada em (8.2) temos que o estimador de máxima verossimilhança do parâmetro $\boldsymbol{\eta}=(\alpha, \theta, \lambda, p)$ é obtido pela resolução do sistema de equações não linerares $\frac{\partial \ell}{\partial \alpha}=0, \frac{\partial \ell}{\partial \theta}=0, \frac{\partial \ell}{\partial \lambda}=0$ e $\frac{\partial \ell}{\partial p}=0$. Isto é feito através da rotina de NewtonRapson do pacote $\mathrm{Ox}$.

As estimativas de máxima verossimilhança são dadas por

$\hat{\alpha}=4,5415(2,4286), \quad \hat{\theta}=0,6735(0,3225), \quad \hat{\lambda}=2,9096(0,5183)$, e $\hat{p}=0,6826(0,0895)$.

Cada valor entre parênteses ao lado das estimativas representa a raiz quadrada do correspondente elemento da diagonal de $i^{-1}(\hat{\alpha}, \hat{\theta}, \hat{\lambda}, \hat{p})$, onde $i(\alpha, \theta, \lambda, p)$ é a matriz de informação observada. Se admitimos a normalidade assintótica para os dados da tabela 8.1, $i^{-1}(\hat{\alpha}, \hat{\theta}, \hat{\lambda}, \hat{p})$ corresponde à estimativa da matriz de covariâncias assintótica, e pode ser utilizada para computar inferências sobres os parâmetros.

Para estudar a possibilidade da utilização do modelo de mistura Weibull para os dados da tabela 8.1. A estatística da razão de verossimilhança para testar $H_{0}: \theta=1$ é $\Lambda=0,21072$ e indica a aceitação do modelo Weibull a um nível de significância 0.05 $\left(\chi_{(1 ; 0,05)}^{2}=3,8441\right)$. Testando o modelo de mistura exponencial $\left(H_{0}: \alpha=1 \theta=1\right)$ temos $\Lambda=27,688$, o que indica que a um nível de significância de $5 \%\left(\chi_{(2 ; 0,05)}^{2}=5,99\right)$, o modelo de mistura exponencial não é adequado para ajuste dos dados. 


\section{Análise Bayesiana para o modelo de mistura Weibull-exponenciada}

Para analisarmos os dados da tabela 8.1 desde uma perpectiva Bayesiana consideramos o modelo de mistura Weibull-exponenciada (WE) com as densidades a priori para $\alpha, \theta, \lambda$ e $p$ dada em (8.16) com $\alpha_{0}=3,24, \alpha_{1}=0,74 \theta_{0}=1,403, \theta_{1}=2,094, \lambda_{0}=32,34, \lambda_{1}=$ $11,15, \gamma_{0}=146,3$ e $\gamma_{1}=62,7$ (a escolha desses valores para os parâmetros da densidade a priori, foram baseadas numa opinião experinte combinada com uma análise preliminar dos dados). A partir da densidades condicionais (8.20), geramos para cada um dos parâmetros do modelo de mistura Wiebull-exponenciada 20 cadeias separads de Gibbs cada uma com 2.000 iterações, e devido ao fato de todas as densidades condicionais marginais (8.20) não apresentarem uma forma padrão conhecida utilizamos então o algoritmo de MetropolisHasting. Com o objetivo de deminuir o efeito de pontos iniciais deprezam-se as 1.000 pimeiras iterações e a partir de daí escolhem-se as iterações de 20 em 20 , ou seja, para cada parâmetro considera-se a 1.020 - ésima, . .,2.000 - ésima iterações; daí as 20 cadeias fornecem um amostra Gibbs de tamanho 2.000 para cada um dos parâmetros. Essa seleção foi realizada de forma a garantir uma baixa correlação entre os elementos da amostra. Esse fato pode ser confirmado através da figura 8.1, que ilustra as funções de autocorrelação das amostras. A convergência das amostras geradas pelo algoritmo de Gibbs com MetropolisHasting foi monitorado utilizando o método proposto por Gelman e Rubin (1992) o qual se baseia na técnica de análise de variância (veja Gelman e Rubin, 1992).

Como pode-se observar na figura 8.1, todos os termos são próximos de zero, indicando que as amostras são não correlacionadas.

Na tabela 8.2, apresenta-se um resumo das distribuições a posteriori marginais aproximada conjuntamente com os estimadores dos fatores de redução de escala potencial $\hat{R}$ (ver por exemplo, Gelman e Rubin, 1992). Observamos na tabela 8.2 , que $\sqrt{\hat{R}}<1.1$, o que nos indica que o algoritmo de Gibbs com Metropolis-Hasting gera para cada parâmetro amostras que convergem em distribuição para as distribuições condicionais marginais (8.20).

$\mathrm{Na}$ figura 8.2 , representa-se as densidades a posteriori marginais aproximadas consi- 

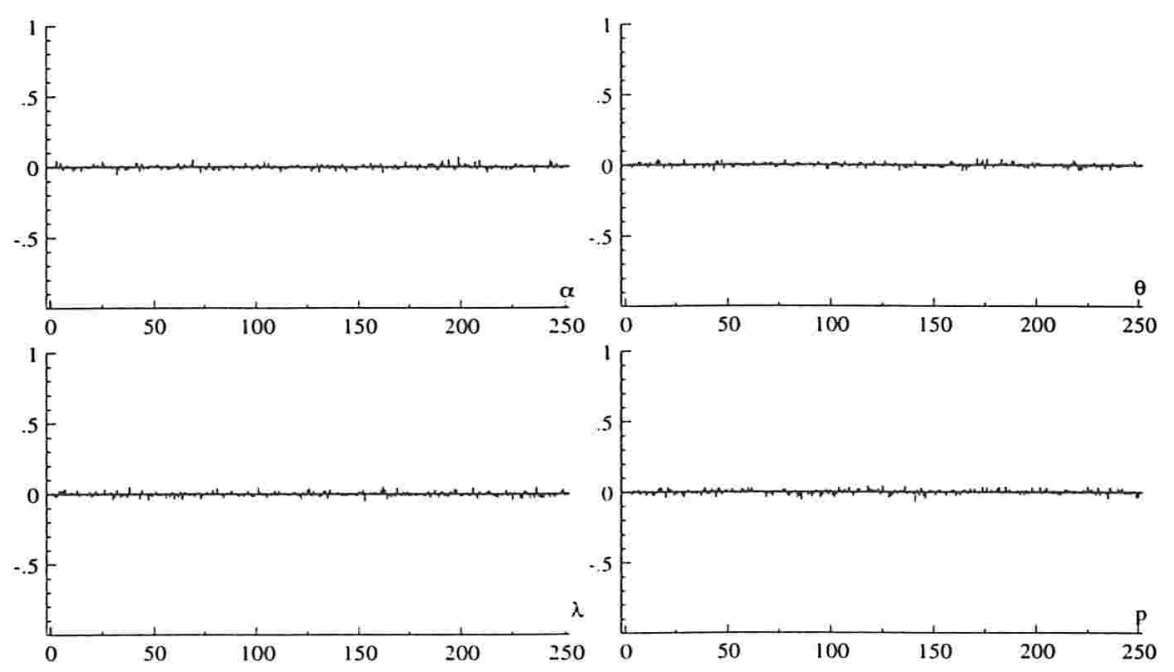

Figura 8.1: Autocorrelação das amostras geradas pelo algoritmo Metropolis-hasting com 2000 pontos

Tabela 8.2: Sumário a posteriori dos parâmetros do modelo de mistura Weibullexponenciada

\begin{tabular}{|llllcc|}
\hline \hline & Média & Mediana & D.P. & Intervalo de credibilidade $95 \%$ & $\hat{R}$ \\
\hline$\alpha$ & 3,9150 & 3,9054 & 0,2896 & $(3,3758 ; 4,5031)$ & 1,01387 \\
$\theta$ & 0,96311 & 0,95632 & 0,11437 & $(0,7616 ; 1,1939)$ & 1.00180 \\
$\lambda$ & 3,0966 & 3,1053 & 0,2436 & $(2,5957 ; 3,5563)$ & 1.00876 \\
$p$ & 0,69977 & 0,70038 & 0,02929 & $(0,6434 ; 0,7568)$ & 1.00019 \\
\hline \hline
\end{tabular}

derando os 1.000 pontos amostrais.

A partir dos resultados da tabela 8.2, podemos considerar o modelo de mistura Weibull para analisar os dados da tabela 8.1, visto que o estimador de Bayes para $\theta$ baseado na aproximação de Monte Carlo para a média a posteriori esta bem próximo de um (observe também que o intevalo de credibildade de $95 \%$ para $\theta$ inclui um), quando $\theta=1 \mathrm{o}$ modelo Weibull-exponenciada (8.1) é o modelo de mistura Weibull. 

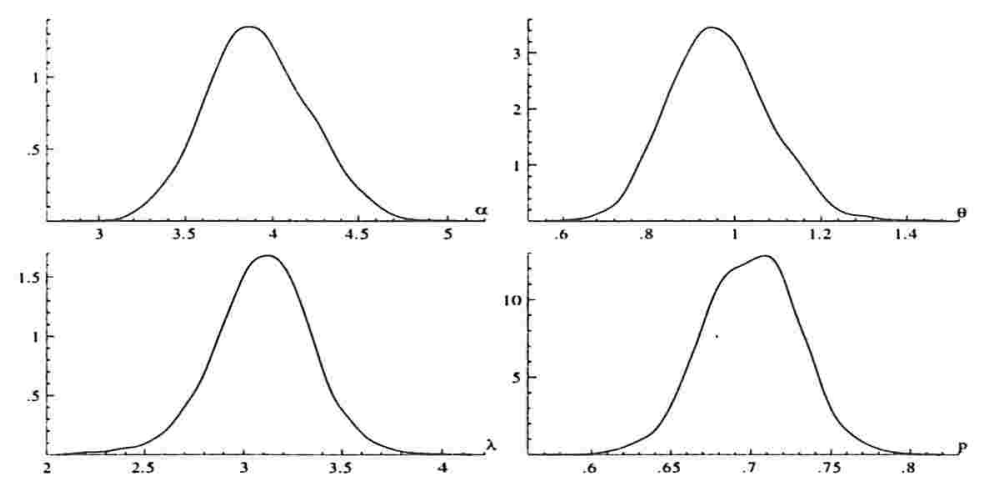

Figura 8.2: Densidades marginais a posteriori aproximados para os parâmetros do modelo de mistura Weibull-exponenciada $\alpha, \theta, \lambda$ and $p$

\section{Análise Bayesiana para o modelo de mistura Weibull}

Para analisar os dados da tabela 8.1, consideramos agora o modelo de mistura Weibull com as densidades a priori (8.21) para os parâmetros, com, $\alpha_{0}=29,17, \alpha_{1}=8,33$, $\lambda_{0}=208,91, \lambda_{1}=67,4$ e $\gamma_{0}=\gamma_{1}=1$

A partir das densidades condicionais marginais (8.23), geramos 20 cadeias separadas de Gibbs cada uma com 2.000 iterações e utilizamos o método proposto por Gelman e Rubir. (1992) para verificar a convergência das cadeias. Para cada um dos parâmetro na cadeia consideramos as iterações 1.020 - ésima, . ., 2.000 - ésima totalizando assim uma amostra Gibbs de 2.000 pontos amostrais.

As quantidades a posteriori de interesse obtidas a partir das amostras selecionadas são dados na tabela 8.3 , onde observamos que os fatores de redução potencial são menores de $1.1(\sqrt{\hat{R}}<1.1)$, indicando a convergência das amostras geradas.

Na figura 8.6, temos as densidades marginais a posteriori aproximadas considerando os 2.000 pontos amostrais. 
Tabela 8.3: Sumário a posteriori dos parâmetros do modelo de mistura Weibull

\begin{tabular}{|llllcc|}
\hline \hline & Média & Mediana & D.P. & Intervalo de credibilidade $95 \%$ & $\hat{R}$ \\
\hline$\alpha$ & 3,4980 & 3,4719 & 0,4595 & $(2,64616 ; 4,44235)$ & 1,09131 \\
$\lambda$ & 3,1723 & 3,1782 & 0,1605 & $(2,64616 ; 4,44235)$ & 1,00671 \\
$p$ & 0,67970 & 0,68115 & 0,03017 & $(0,617727 ; 0,735598)$ & 1,00038 \\
\hline
\end{tabular}

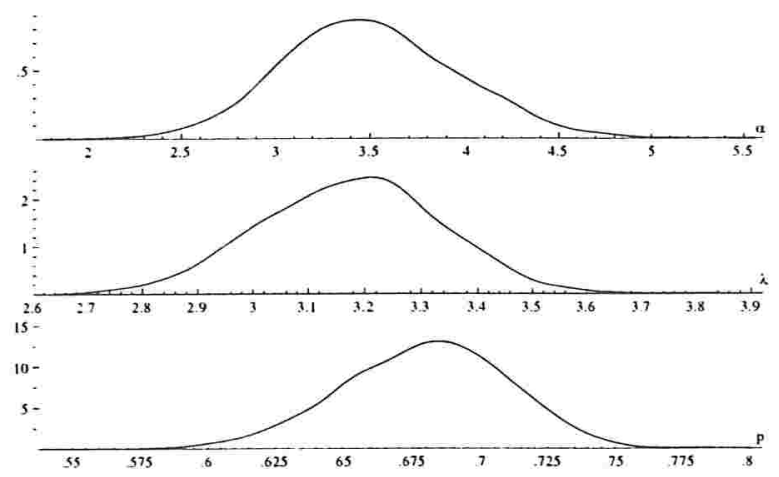

Figura 8.3: Densidades marginais a posteriori aproximados para o modelo de mistura Weibull $\alpha, \lambda$ and $p$

\section{Teste de hipóteses Bayesiano}

A estimativa de Monte Carlo da verossimilhança preditiva baseada nas amostras Gibbs dos parâmetros do modelo de mistura Weibul-exponenciada (modelo sob $H_{1}$ em (8.12)) resultou em $\hat{f}\left(D \mid M_{1}\right)=0,328767$.

Para o modelo sob $H_{0}$ dado em (\$.12)(modelo de mistura Weibull) uma estimativa da verossimilhança priditiva baseada nas amostras de Gibbs dos parâmetros do modelo Weibull resultou em $\hat{f}\left(D \mid M_{0}\right)=1,13166$. O pseudo-fator de Bayes do modelo de mistura Weibull com respeito ao modelo Weibull-exponenciada é $P S F B_{01}=3,464806$. Conclui-se que o modelo de mistura Weibull é adequado para o ajuste dos dados da tabela 8.1. A mesma conclusão foi obtida com o procedimento clássico. 
Para testar se o modelo exponencial é adequado para o ajuste dos dados da tabela 8.1 desde a perspectiva Bayesiana, calculamos a verossimilhança preditiva que resultou em $\hat{f}\left(D \mid M_{2}\right)=3,0809776 \times 10^{-6}$. O pseudo-fator de Bayes do modelo exponencial com respeito ao modelo Weibull-exponenciada é $P S F B_{21}=9,37129 \times 10^{-6}$, indicando a rejeição do modelo exponencial para o ajuste dos dados da tabela 8.1 , confirmando assim a conclução obtidas com o método clássico.

Na figura 8.4, plotamos o log a razão dos CPO para os diferentes modelos com respeito ao número de observações. Valores positivos do log da razão dos CPO indica a preferência pelo primeiro modelo. Por exemplo, na figura 8.4, o gráfico para o modelo de mistura Weibull versus do modelo de mistura Weibull-exponenciada indica que 16 das 30 observações suportam o modelo de mistura Weibull sobre o modelo de mistura Weibull-exponenciada. Similarmente, 18 observações sustentam o modelo de mistura de Weibull-exponenciada sobre o modelo de mistura exponencial.
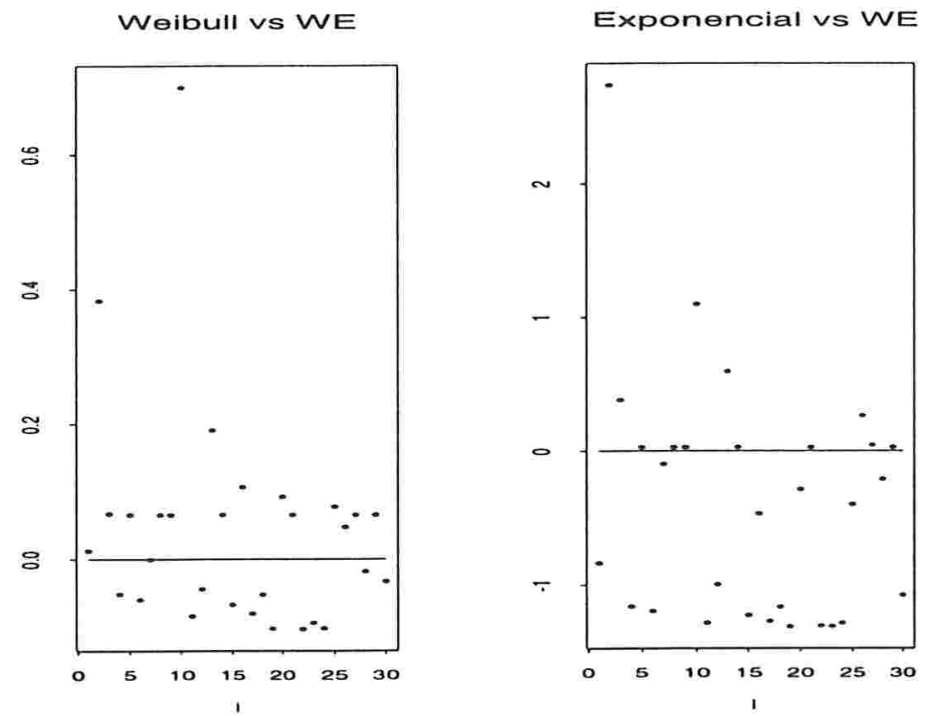

Figura 8.4: Gráfico da razão de CPO para comparar os diferentes modelos versus observações 


\subsubsection{Exemplo 2-Dados de Kersey et al. 1987}

Nesta subeção apresentamos um exemplo como aplicação dos procedimentos clássicos e bayesianos, discutidos nas seções anteriores. O exemplo consiste em analisar os dados (veja tabela 6.6) obtidos por Kersey et al. (1987), os quais se referem aos tempos de recaída de pacientes de leucemia submetidos a dois tipos de transplante, "allogeneic" (Grupo 1) ou "autologous" (Grupo 2).

\section{Análise clássica}

Nesta subseção apresenta-se uma aplicação da métodologia discutida na seção 2. Considerandose apenas os dados do Grupo 2 da tabela 6.6. Para estimar os parâmetros, consideramos o mesmo procedimento dado antes.

Como resultado as estimativas de máxima verossimilhança são dadas por

$$
\hat{\alpha}=0,42002(0,25013), \hat{\theta}=44,452(20,56), \hat{\lambda}=104.13(15,58), \hat{p}=0.79629(0,0608) \text {. }
$$

Para estudar a possibilidade de utilizar o modelo de mistura Weibull para os dados grupo 2 da tabela 6.6, testa-se $\left(H_{0}: \theta=1\right)$ e temos que a estatística da razão de verossimilhanças (8.13), resultou em, $\lambda=11.083$ e este resultado implica que o modelo mistura Weibull não é estatísticamente adequada para ajustar os dados do grupo 2 da tabela 6.6 ao nível de significância de $5 \%,\left(\chi_{1,0,05}^{2}=3,8441\right)$.

Testando o modelo de mistura exponencial $\left(H_{0}: \alpha=1 \theta=1\right)$ temos $\Lambda=17,2112$, o que indica que a um nível de significância de $5 \%\left(\chi_{(2 ; 0,05)}^{2}=5,99\right)$, o modelo de mistura exponencial não é adequado para o ajuste dos dados.

\section{Análise Bayesiana para o modelo de mistura Weibull-exponenciada}

Para analisarmos os dados do Grupo 2 da tabela 6.6 desde uma perpectiva Bayesiana consideramos o modelo de mistura Weibull-exponenciada (WE) com as densidades a priori 
para $\alpha, \theta, \lambda$ e $p$ dada em (8.16) com $\alpha_{0}=1, \alpha_{1}=0,00000002 \theta_{0}=1, \theta_{1}=0,00000002$, $\lambda_{0}=1, \lambda_{1}=0,0000002, \gamma_{0}=1$ e $\gamma_{1}=1$ (observe que a densidade a priori é não informativa). A partir da densidades condicionais (8.20), geramos para cada um dos parâmetros do modelo de mistura Wiebull-exponenciada 20 cadeias separadas de Gibbs cada uma com 2.000 iterações, e devida ao fato de todas as densidades condicionais marginais (8.20) não apresentarem uma forma padrão conhecida utilizamos então o algoritmo de MetropolisHasting. Com o objetivo de deminuir o efeito de pontos iniciais deprezam-se as 1.000 pimeiras iterações e a partir de daí escolhem-se as iterações de 20 em 20 , ou seja, para cada parâmetro considera-se a 1020 - ésima, .., 2000 - ésima iterações; daí as 20 cadeias fornecem um amostra Gibbs de tamanho 2.000 para cada um dos parâmetros. A convergência das amostras geradas pelo algoritmo de Gibbs com Metropolis-Hasting foi monitorada utilizando o método proposto por Gelman e Rubin (1992).

Na tabela 8.4, apresenta-se um resumo das distribuições a posteriori marginais aproximadas conjuntamente com as estimativas dos fatores de redução de escala potencial $\hat{R}$. Observamos na tabela 8.4, que $\sqrt{\hat{R}}<1,1$, o que nos indica que o algoritmo de Gibbs com Metropolis-Hasting gera para cada parâmetro amostras que convergem em distribuição para as distribuições condicionais marginais (\$.20).

Tabela 8.4: Sumário a posteriori dos parâmetros do modelo de mistura Weibullexponenciada

\begin{tabular}{|ccllcc|}
\hline \hline & Média & Mediana & D.P. & Intervalo de credibilidade $95 \%$ & $\hat{R}$ \\
\hline$\alpha$ & 0,4191 & 0,4188 & 0,0129 & $(0,3939 ; 0,4455)$ & 1,000387 \\
$\theta$ & 44,216 & 44,172 & 1,715 & $(40,919 ; 47,650)$ & 1,00781 \\
$\lambda$ & 104,33 & 104,29 & 2,216 & $(100,056 ; 108,866)$ & 1.00626 \\
$p$ & 0,7923 & 0,7931 & 0,02811 & $(0,7340 ; 0,8441)$ & 1,00156 \\
\hline \hline
\end{tabular}

Na figura 8.5 , representa-se as densidades margimais a posteriori aproximadas consideranda os 1.000 pontos amostrais. 

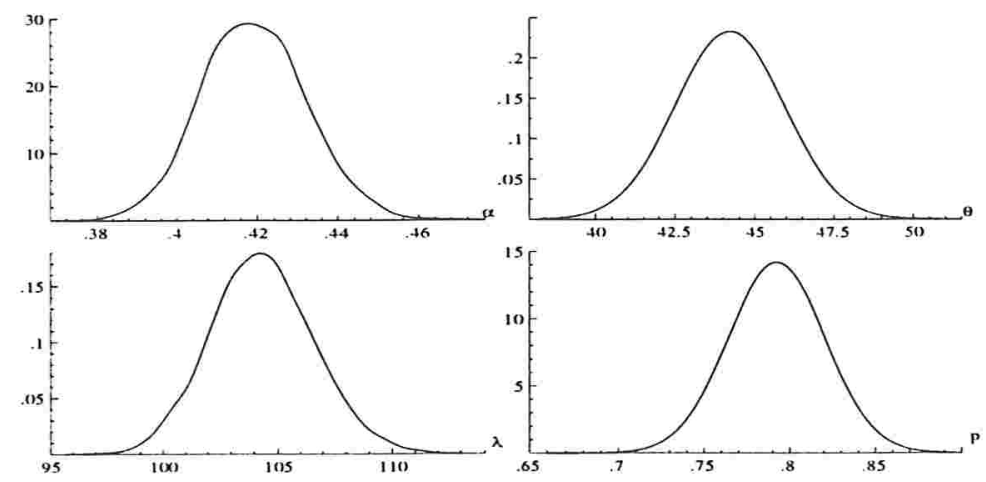

Figura 8.5: Densidades a posteriori margial aproximados para os parâmetros do modelo de mistura Weibull-exponenciada $\alpha, \theta, \lambda$ and $p$

\section{Análise Bayesiana para o modelo de mistura Weibull}

Para analisar os dados da tabela 8.1, consideramos agora o modelo de mistura Weibull com as densidades a priori (8.21) para os parâmetros, com, $\alpha_{0}=1, \alpha_{1}=0,00000002$, $\lambda_{0}=1, \lambda_{1}=0,0000002$ e $\gamma_{0}=\gamma_{1}=1$

A partir das densidades condicionais marginais (8.23), geramos 20 cadeias separadas de Gibbs cada uma com 2.000 iterações e utilizamos o

método proposto por Gelman Rubin (1992) par verificar a convergência das cadeias. Para cada um dos parâmetro na cadeia consideramos as iterações 1020 - ésima, . ., 2000 ésima totalizando assim uma amostra Gibbs de 2.000 pontos amostrais.

As quantidades a posteriori de interesse obtidas a partir das amostras selecionadas são dadas na tabela 8.5 , onde observamos que os fatores de redução potencial são menores de $1.1(\sqrt{\hat{R}}<1,1)$, indicando a convergência das amostras geradas.

Na figura 8.6, temos as densidades marginais a posteriori aproximadas considerando os 2.000 pontos amostrais. 
Tabela 8.5: Sumário a posteriori dos parâmetros do modelo de mistura Weibull

\begin{tabular}{|ccllcc|}
\hline \hline & Média & Mediana & D.P. & Intervalo de credibilidade $95 \%$ & $\hat{R}$ \\
\hline$\alpha$ & 1,3516 & 1,3490 & 0,1368 & $(1,09102 ; 1,62325)$ & 1,000471 \\
$\lambda$ & 2,4309 & 2,4230 & 0,2671 & $(1,9328 ; 2,9882)$ & 1,000914 \\
$p$ & 0,7911 & 0,7917 & 0,02836 & $(0,7329 ; 0,8441)$ & 1,0000173 \\
\hline
\end{tabular}

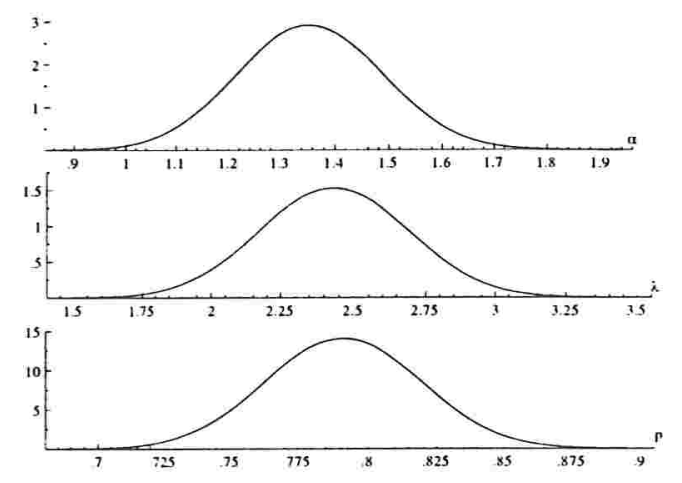

Figura 8.6: Densidades a posteriori aproximados para o modelo de mistura Weibull $\alpha, \lambda$ and $p$

\section{Teste de hipóteses Bayesiano}

A estimativa de Monte Carlo da verossimilhança preditiva baseada nas amostras Gibbs dos parâmetros do modelo de mistura Weibul-exponenciada (modelo sob $H_{1}$ em (8.12)) resultou em $\hat{f}\left(D \mid M_{1}\right)=2,953 \times 10^{-7}$.

Para o modelo sob $H_{0}$ dado em (8.12)(modelo de mistura Weibull) uma estimativa da verossimilhança priditiva baseada nas amostras de Gibbs dos parâmetros do modelo Weibull resultou em $\hat{f}\left(D \mid M_{0}\right)=1,681 \times 10^{-12}$. O pseudo-fator de Bayes do modelo de mistura Weibull com respeito ao modelo Weibull-exponenciada é $P S F B_{01}=5,6925 \times$ $10^{-6}$. Conclui-se que o melhor modelo é a mistura Weibull-exponenciada para os dados do grupo 2 da tabela 6.6. A mesma conclusão foi obtida com o procedimento clássico. 
Para testar se o modelo exponencial é adequado para o ajuste dos dados da tabela 6.6 desde a perspectiva Bayesiana, caculamos a verossimilhança preditiva que resultou em $\hat{f}\left(D \mid M_{2}\right)=1,306 \times 10^{-10}$. O pseudo-fator de Bayes do modelo exponencial com respeito ao modelo Weibull-exponenciada é $P S F B_{21}=0,00044$, indicando a rejeição do modelo exponencial para o ajuste dos dados do grupo 2 da tabela 6.6, confirmando assim as conclusões obtidas com o método clássico.

Na figura 8.7, plotamos o log a razão dos CPO para os diferentes modelos com respeito ao numero de observações. Valores positivos do log da razão dos CPOs indica a preferência do primeiro modelo. Por exemplo, na figura \$.7, o gráfico para o modelo de mistura Weibull versus do modelo de mistura Weibull-exponenciada indica que 26 das 44 observações suportam o modelo de mistura Weibull-exponenciada sobre o modelo de mistura Weibull. Similarmente, 38 observações sustentam ao modelo de mistura de Weibull-exponenciada sobre o modelo de mistura exponencial.
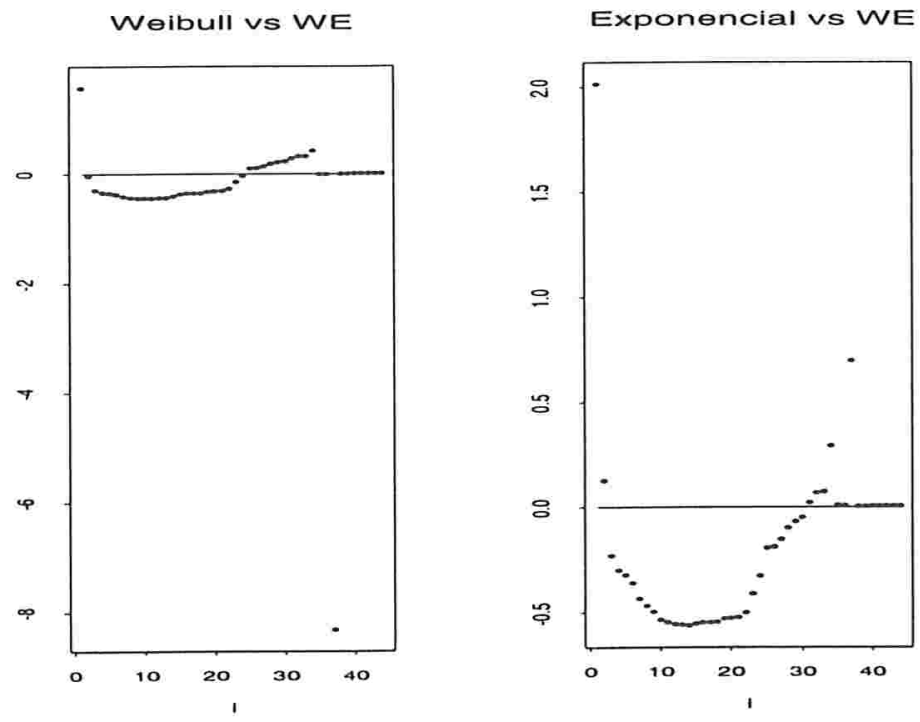

Figura 8.7: Gráfico da razão de CPO para comparar os diferentes modelos versus as observações 


\section{Conclusões}

Finalizamos este trabalho, acreditando que a utilização de métodos Bayesianos aplicados à análise de dados de vida, baseados em métodos de simulação via Cadeias de Markov (MCMC), pode ser uma alternativa importante às tècnicas de análise usuais.

Os algoritmos relacionados ao procedimento MCMC (algoritmo de Gibbs e MetropolisHasting) são de fácil implementação e não exigem um conhecimento computacional avançado, e, os resultados parecem ser mais precisos que os resultados do método de máxima verossimilhança. Portanto, podem ser usados de forma segura, além de facilitar os cálculos na inferência dos parâmetros do modelo.

Resumimos, a seguir, os principais resultados obtidos neste trabalho.

(i) Desenvolvimento da metodologia Bayesiana para o modelo Weibull-exponenciada proposto por Mudholkar et al. (1995).

(ii) Consideração do modelo de regressão Weibull-exponenciada. Este modelo estende o modelo proposto por Mudholkar para modelos com covariáveis.

(iii) Desenvolvemos uma análise Bayesiana alternativa baseada em métodos MCMC, para o modelo proposto por Rodrigues, Bolfarine e Louzada-Neto (1993), para comparar populações exponenciais com dados acelerados.

(iv) Desenvolvemos uma metodologia Bayesiana para comparar a distribuição Weibullexponenciada com várias famílias de distribuições usadas para o ajuste de dados 
vida com taxas de falha do tipo "bathtub".

(v) Desenvolvemos uma metodologia Bayesiana para os modelos de mistura exponencial proposta por Githany et al. (1992).

(vi) Desenvolvemos uma metodologia Bayesiana para o modelo em (v) com covariáveis associadas aos parâmetros do modelo de mistura exponencial.

(vii) Propomos o modelo de mistura Weibull-exponenciada e utilizamos este modelo para estudar a adequabilidade dos modelos de mistura exponencial e Weibull.

(viii) Desenvolvimento de uma metodologia para testar ordenação na comparação da proporções de sobreviventes quando vários tratamentos são considerados. Acreditamos que esta metodologia pode ser extendida para as prioris consideradas em Chen (1994).

Acreditamos que as técnicas desenvolvidas neste trabalho também podem ser aplicadas a outros modelos de sobrevivência ou confiabilidade, como os modelos de regressão Weibull-exponenciada com erros de medição nas covariadas e modelos de mistura Weibullexponenciada com covariáveis, seja com ou sem erros de medição nas covariadas. 


\section{Bibliografia}

[1] Abramowitz, M. e Stegun. I.A. (1965). Handbook of Mathematica functions. New York: Dover

[2] Achcar, J. A. and Bolfarine, H. (1986) The log-linear model with a generalized Gamma distribuition for the Error: A Bayesian approch. Statistics and Probability Letters, $4325-332$.

[3] Achcar, J.L, and Louzada-Neto, F. (1992) A Bayesian approach for accelereted life tests considering the Weibull distribution, Computational statistical, 7, 355-369.

[4] Achcar, J.A. and Pereira, G. A. (1998) Use of mixture of exponential power distributions for intervals-censored survival data in presence of covariates. Notas do ICMSC.

[5] Aitkin, M. (1991). Posterior factor. J. R. Stat. Soc., B, 53, 111-142.

[6] Anscombe, F. J. (1961). Estimating a mixed-exponential reposte law. J. Amm. Statist. Ass., 56 493-502.

[7] Arset, M. V. (1987). "How to identify bathub hazard rate", IEEE Trans. Reliability,36 106-108.

[8] Berger, J. O. and Perechi, L. R. (1992). The intrinsic Bayes factor. Techneical report. Departament of statistics, Pardue University, Wes Lafayette.

[9] Beerkson, J. and Gage, R.P. (1952). survival cure for cancer petients following tratment, J. Amer. Statist. Ass., bf 47 501-515. 
[10] Bedrick, E.J., Christensen. R. and Johnson, W. (1996). J. Amer. Statist. Ass., bf 91 1450-1460.

[11] Boag, J.W. (1949) Maximum likelihood estimates of the proportion of patients cured by cancer therapy, J. R. Stat. Soc., B, 15-44.

[12] Box, G. E. P. (1980). Sampling and Bayes inference in scientific modeling and Robustness. J. R. Stat. Soc., A, 143, 383-430.

[13] Box G. E. P. and Tiao, G. C. (1973) Bayesian inference in statistical analysis., Addison-Wesley: Reading, Mass.

[14] Broadhurst, R. G. and Maller, R. A. (1990) The recidivism of prisoners released for the first time: reconsidering the effectiveness questions. Ast. N. Z. J. Crim. 23, SS-103.

[15] Carlin, B. P. and Gelfand, A. E. (1993). Explaining the Gibbs sampler. Amer. Statistician, 46, 167-174

[16] Chen, M.-H. (1994). Importance-weighted marginal Bayesian posterior density estimation. J. Amer. Statist. Assoc. , 89, 8818-824.

[17] Chib, S.and Greenberg, E. (1995). Understanding the Metropolis-Hasting. Amer. Statistician, 49, 327-335

[18] Collet, D. (1994). Modelling survival data in medical research. Chapman and Hall, London.

[19] Cowles, M.K., and Carlin, B.P. (1996) Markov chain Monte Carlo convergence diagnostics: A comparative review. J. Amm. Statist. Ass., bf 91 \$83-905.

[20] Cox, D.R., and Oakes, D. (1984). Analysis of Survival Data. London: Chapman and Hall. 
[21] Dempster, A., Laird, N. and Rubin, D. (1977). Maximum likelihood from incomplete data via the EM algorithm. J. R. Statist. Soc., B, 39, 1-38.

[22] Devroye, L. (1986).Non-Uniform Random Variate Generation. Springer-Verlag. New York.

[23] Dunsmuir, W., Tweedie, R., Flanck, L. and Mengersian, K. (1989). Modelling of transitions between employment sattes for young Australians, austral. J. Statist., 31A, 165-196.

[24] Doornik, J. (1996). Ox: An object-oriented matrix Programming language. International Thomsom Business Press.

[25] Doornik, J., Draisma, G. and Ooms, M. (1998). Ox tutorial http://www.nuff.ox.ac.uk/Users/Doornik.

[26] Fahrmeir, L. and Kaufmann, h. (1985). Consistency and asymptotic normality of the maximum likelihood estimator in generalized models, Ann. Statist.,13, 342-368.

[27] Farewell, V. T. (1977a). A model for a binary variable with time censored observations, Biometrika, 64, 43-46.

[2S] Farewell, V. T. (1977b). The commbined effect of breast cancer risk factors. Cancer, 40, 931-936.

[29] Farewell, V. T. (1982). The use of mixture models for the analysis of survival data with long-term survivors, Biometrics 38, 1041-1046.

[30] Farewell, V. T. (1986). Mixture models in survival anlysis: are they worth the risk? Canad. J. Statist. 14, 257-262.

[31] Feigel, P. amd Zelen, M. (1965). Estimation of exponential survival probabilities with concomitant information. Biometrics, 21 826-\$34. 
[32] Geisser, S., Eddy, W. (1979). A predicitve approach to model selection. J. Amer. Statist. Assoc., 79, 153-160.

[33] Gamerman, D. (1996). Simulação estocástica via Cadeias de Markov. 12 SINAPECAXAMBU-29/07 a 02/08/96.

[34] Gelfand, A.E., Dey, D.K., and Chang, H. (1992). Model determination using predictive ditributions with implementation via sampling-based methods (with discussion), in Bayesian Statistics 4, eds. J.M. Bernardo, J.O Berger, A. P Dawid, and A.F.M. Sith, Oxford:Oxford University Press, pp. 147-196.

[35] Gelfand, A. E. and Dey, D. K. (1994). Bayesian model choice: Asymptotics and exact calculations. J. Roy. Statist. Soc., Ser. 56, 501-514.

[36] Gelfand, A. E. and Smith, A. F. M. (1990). Sampling-based approaches to calculating marginal densities. J. Amer. Statist. Assoc., 85, 398-409.

[37] Gelfand, A. E., Smith, A. F. M., and Lee, T. M. (1992). Bayesian anlysis of constrained parameter and truncated data problems using Gibbs samplig. J. Amer. Statist. Assoc., 87, 398-409.

[38] Geman, S., and Geman, D (1984). Stochastics relaxation, Gibbs distribuitions and the Bayesian restoration of images. IEEE Trans. on Pattern Analysis and Machine Intelligence, 6, 681-\$89.

[39] Gelman, A. and Rubin,. D. B. (1992). Inference from iterative simulating using multiple sequences (with discussion).Statistical Science, 7, 457-511.

[40] Geman, S. and Geman, D. (1984). Stochastic relaxation, Gibbs distributions and the Bayesian restoration of images. IEEE Trans. on Pattern Analysis and Machine Intlligence, 6, 721-741.

[41] Ghitany, M. E. (1993) On the information matrix of exponential mixture models whit long-term survivors, Biom. J., 35, 15-27. 
[42] Ghitany, M. E. and Maller. R. A. (1992). Asymptotic results for exponential mixture models whit long-term survivors and covariates, J. Mult. Analysis, 49, 218-241.

[43] Ghitany, M. E., Maller, R. A. and Zhou, S. (1995). Exponential mixture models whit long-term survivors, Statistics, 23, 231-336.

[44] Ghitany, M. E. and Maller, R. A. (1995). Estimating the proportion of immunes in censored samples: a simulation study, Statistics in Medicine 14, 39-49.

[45] Glasser, R.E. (1980). Bathtub and realted failure rate characterization. J. Amer. Statist. Assoc., 75 667-672.

[46] Glasser, M. (1967). Exponential survival with covariance. J. Am. Statist. Assoc., 62 651-568.

[47] Goldman, A.I. (1984) Survivorship analysis when cure is a possibility: a Monte Carlo study, Statistics in Medicine, 3 153-163.

[48] Greenhouse, J. B. and Wolfe, R. A. (1984) A competing risks derivation of a mixture model for the analysis of survival data. Comm. Statist.-Theor. Meth., 13 3133-3154.

[49] Kersey, J.H., Weisdorf, D.A., Nesbit, M.E., LeBien, T.W., T.W., Woods, W.G., McGlave, P.B., Kim, T.. Vallera, D.A., Goldaman, A.I.,Bostrom,B., Hurd, D. and Ramsay, N.K.C. (1987). Comparison of autologous and allogenec bone marrow transpaltation for treatment of high-risk refractory acute lymhoblastic leukaemia, New England Journal of Medicine, 317, 461-467.

[50] Hasting, W.K. (1970). Monte Carlo sampling methods using Markov chains and their aplications. Biometrika, 57, 97-109.

[51] Hjort, U. (1980). A realiability distributions with increasing, decreasing, constant and bathtub failure rates. Technometrics, 22, 99-107.

[52] Hsu, D. A. (1982). A Bayesian robust detection of shift in the risk structure of stock market returns. J. Amer. Statist. Assoc., 77, 29-39. 
[53] Laird, N. and Ware, J. H. (1982). Random-effects models for longitudinal data. Biometrics 38, 936-974.

[54] Lawles, J.F. (1982). Statistical Models and Methods for Lifetime Data, New York: John Wiley.

[55] Leibelein. J. and Zelen, M. (1956). Statistical investigation of the fatigue life of deep groove ball bearing, J. Res. Nat. Bur. Stand., 57 273-316.

[56] Louzada-neto, F., Bolfarine, H. e Rodrigues, J. (1991). Comparação de dois modelos exponenciais com dados acelerados: uma abordagem Bayesiana. R. Bras. Estat., 52 93-103.

[57] Kass, R. E. and Raftery, A. E. (1995). Bayes factor. J. Amer. Statist. Assoc., 90, 773-795.

[58] Kuo, L. e Yang, T. (1995). Bayesian Computation of software reliability. Journal of computational and Graphical Statistics, 4 65-82.

[59] Maltz, M. D. (1984). Recidivism. Academic Press, New York.

[60] Maltz, M. D. And MacCleary, R. (1977). The mathemathical of behavioral change: recidivism and construct validity; Evaluation Quarterly, 1,421-438.

[61] Maller, R.A. (1988) On the exponential model for survival, Biometrika, 75, 582-586.

[62] Maller, R.A. and Zhou, S. (1992) The probability that the largest observation is censored, J. Appl. Prob.,, 30, 602-615.

[63] Maller, R.A. and Zhou, S. (1993) Estimating the proportion of immunes in a censored sample, Biometrika, 79, 731-739.

[64] Maller, R.A. and Zhou, X. (1996). Survival analysis with long-term survivors, New York: John Wiley \& Sons. 
[65] Mccullagh, P., and Nelder, J. (1992). Generalized Linear Models, London; Chapman and Hall.

[66] Meeker, Jr. W. Q. (1987). Limited failure population life test: aplications to integrated circuit reliability. Technometrics $29,51-65$.

[67] Metropolis, N., Rosenbluth, A.W., Rosenblut, M.N., Teller, A.H., and Teller, E. (19.53), Equation of state Calculations by Fast Computing Machines, Journal of chimical physics, 21, 1087-1092.

[68] Mudholkar, G.S., Srivastava, D.K., and Friemer,M. (1995) The exponentiated Weibull family: A reanalysis of the bus-motor-failure data. Technometrics $37,436-445$.

[69] Nelson, W. B. (1990). Accelereted Testing; statistical Models, test plans and data analysis. New York: John Wiley.

[70] Nelson, W.B. (1972). Graphical analysis of accelerated test data with the inverse power law, IEE Trans. Realiab., R21 2-11.

[71] Naylor, J. C. and Smith, A. F. M. (1982). Applications of a method for the efficient computation of posterior Distributions. Applied Statistics, 31, 214-225.

[72] Newton, M. A. and Raftery, A. E. (1994). Approximate Bayesian inference by the weighted likelihood bootstrap (with discussion). J. Roy. Statist. Soc. Ser. B 56, 1-48.

[73] Peto, R., Lee, P.N. and Paige, W.S. (1972). Statistical analysis of the bioassy of continuous carcinogens. Br. J. Cancer, 26 258-261.

[74] Pike, M.C. (1966). A method of anlysis of a certain class of experiments in carcinogenesis. Biometrics, 35, 142-161.

[75] Pocock, s.J., Gore, S. M. and Kerr, G. (1982). Long-term survival analysis: the curability of breast cancer, Statistics in Medicine, 1, 93-104. 
[76] Prentice, R.L. (1975). Discrimation among some parametric models. Biometrika, 61, $539-544$.

[77] Raftery, A. E. (1987). Inference and prediction for a general order statistic modelo with unknown population size, J. Amer. Statist. Assoc., 82, 1163-1168.

[78] Raftery, A. E. (1995). Hipothesis testing and selections. Em Markov Chain Monte Carlo in pratice (Eds: W. R. Gilks, S. Richardson and D. J. Spielgelhater), chap. 10. New York: Marcel Dekker.

[79] Rajarshi, S. and Rajarshi, M.B (1988). Bathtub distributions: a review. Commun. Statist.- Theory Meth., 17(8), 2597-2621.

[80] Rodrigues, J,; Bolfarine, H.; Neto, F.L. (1993). Comparing several accelerated life models. Comm. Statist.-Theory Meth. 22(8), 2297-2308.

[81] Rodrigues, J. (1998). Bayesian Analysis for the accelerated life tests with informative prior distributions obtained from fixed stresses. Notas do ICMSC-USP-São Carlos.

[82] Stacy, E. W. (1962). A generalization of the gamma distributions. Ann. Mathe. statist. , 33 1187-1192.

[83] Smith, A.F.M. and Roberts, G.O. (1993). Bayesian computation via the Gibbs sampler and related Markov Chain Monte Carlo Methods, J. Roy. Statist. Soc., Ser. B, $\mathbf{5 5}, 3-23$.

[84] Smith, R. M. and Bain, L. J. (1975) An exponential power life-testing distribution. Commun. in Statist., 469-481.

[85] Smith, R. M. and Bain, L. J. (1976). correlation-type goodness of fit sattistics with censored sampling. Commun. in Statist., 469-481.

[86] Spiegelhater, D. J. and Smith, A. F. M. (1982). Bayes factor for linear and log-linear models with vague prior information, J. R. Statist. Soc., Ser. B, 44, 377-387. 
[87] Stone, M. (1974). Cross-validatory choice and assessment of statistical predictions (with discussion). J. Roy. Stat. Soc. Ser B, 36, 111-147.

[88] Struthers, C.A. and Farewell, V. T. (1989). A mixture model for time to AIDS data with left truncation and an uncertain origin, Biomentika. 76, 814-817.

[89] Tanner, M, and Wong, W. (1987). The calculating of posterior distributions by data augmentation J. Amer. Statist. Assoc., 82, 528-550.

[90] Tierney, L. and Kandane, J.B. (1986). Accurate approximations for posterior moments and marginal densities, J. Amer. Stat. Ass. 81 82-86.

[91] Tierney, L. (1994). Markov chains for exploring posterior distributions (with discussion). Ann. Statist., 1701-1762.

[92] Yamaguchi, K. (1992). Accelerated failure time regression models with a regression model of surviving fraction: An application to the analysis of "permanent employment", J. Amer. Statist. Assoc., 87, 284-292.

[93] Zhou, S. and Maller, R.A. (1995). The likelihood ratio test for the presence of immunes in a censored sample, Statistics, 27 181-201. 


\section{Apêndice A}

\section{Critério de convergência}

Para verificarmos se as amostras geradas pelos algoritmos de Gibbs com MetropolisHasting estão realmente converjindo para uma distribuição estacionária, utilizamos neste trabalho o método, proposto por Gelman e Rubin (1992), o qual considera várias cadeias em paralelo partindo de valores iniciais distintos. Para cada parâmetro de interesse comparamos, a variabilidade dentro e entre as cadeias amostradas. Uma vez atingida a estacionaridade, digamos na $i$-ésima iteração, consideramos as realizações $\left(\theta_{j}, \theta_{j+h}, \ldots, \theta_{j+n h}\right)$, $j \geq i$ como uma amostra aleatória da distribuição desejada. Devemos considerar $h$ razoavelmente grande de tal forma que 2 valores sucessivos de $\theta$ sejam aproximadamente independentes; assim teremos uma amostra independente e identicamente distribuído (iid) da distribuição de interesse.

A convergência é monitorada através do fator $\hat{R}$, introduzido por Gelman e Rubin (1992) e para tanto, consideremos $m \geq 2$ cadeias de comprimento $2 n$. No caso de multimodal é recomendável inicializar pelo menos uma cadeia a partir de cada moda. Com as $n$, últimas observações, calculamos:

1. $E$ : a variância entre as $m$ cadeias:

$$
E=\frac{n}{m-1} \sum_{i=1}^{m}\left(\bar{\theta}_{i .}-\bar{\theta}_{. .}\right)^{2}
$$


onde $\bar{\theta}_{i}$. é a média baseada nas $n$ últimas iterações da cadeia $i, i=1, \ldots, m$ e $\bar{\theta}$.. é a média de $\bar{\theta}_{j .}, \ldots, \bar{\theta}_{m}$.

2. $D$ : variância dentro das cadeias dadas por

$$
D=\frac{\sum_{i=1}^{m} s_{i}^{2}}{m}
$$

sendo $s_{i}^{2}=\frac{\sum_{j=1}^{n}\left(\theta_{i j}-\bar{\theta}_{i .}\right)^{2}}{n-1}$.

3. Sob convergência, todos os $n m$ valores são gerados da posteriori e a variância da distribuição a posteriori pode ser estimada de forma não viciada por

$$
\hat{\sigma}^{2}=\frac{1}{n} E+\frac{n-1}{n} D
$$

e sua média é estimada por $\hat{\mu}=\bar{\theta}_{\text {.. }}$.

Resultado então que $\pi(\theta \mid D)$ tem uma distribuição aproximadamete $t$-student com centro em $\hat{\mu}$, escala $\sqrt{\hat{V}}=\sqrt{\hat{\sigma}^{2}+E / m n}$ e graus de liberdade $d f=\hat{V}^{2} / v \hat{a} r(\hat{V})$ onde

$$
\begin{aligned}
\operatorname{var}(\hat{V})= & \left(\frac{n-1}{n}\right)^{2} \frac{1}{m} \operatorname{vâr}\left(s_{i}^{2}\right)+\left(\frac{m+1}{m n}\right)^{2} \frac{2}{m-1} E^{2}+2 \frac{(m+1)(n-1)}{m n^{2}} \\
& \times \frac{n}{m}\left[\operatorname{covv}\left(s_{i}^{2}, \bar{\theta}_{i .}^{2}\right)-2 \bar{\theta}_{. .} \operatorname{cov}\left(s_{i}^{2}, \bar{\theta}_{i .}\right)\right]
\end{aligned}
$$

e onde a variância e covariância são estimadas a partir dos $m$ valores amostrais de $\bar{\theta}_{i \text {. }}$ e $s_{i}^{2} ; d f \rightarrow \infty$ quando $n \rightarrow \infty$.

Um indicador da convergência da simulação iterativa é dada pelo fator redução de escala potencial dada por:

$$
\sqrt{\hat{R}}=\sqrt{\frac{\hat{V}}{D} \frac{d f}{d f-2}},
$$

esperando que esta quantidade convirja para 1 à medida que o número de iterações tende para o infinito $(n \longrightarrow \infty)$. Caso isto não ocorra, devemos considerar mais simulações para melhorar a inferência sobre a distribuição de interesse, ou seja, até que $\sqrt{\hat{R}} \approx 1$. 


\section{Apêndice B}

\section{Alguns programas}

Neste apêndice, apresentamos alguns programas computacionais utilizados neste trabalho. Os programas foram desenvolvidos utilizando a Procedure IML (interactive matrix languaje) do software SAS versão 6.11.

1. Modulos para obtenção das amostras de $\alpha, \theta$ e $\sigma$ no modelo de Weibull-exponenciada, para os dados de Efron (1988);

PROC IML;

START INI;

USE EFRON;

READ ALL VAR\{TEMPO CENSURA\};

$\mathrm{T}=\mathrm{TEMPO} / 30.438$;

$\mathrm{C}=\mathrm{CENSURA}$

$\mathrm{N}=\mathrm{NROW}(\mathrm{T})$;

$\mathrm{R}=\mathrm{C}[+]$;

$\mathrm{S} 0=\mathrm{SUM}(\mathrm{C} \#((\mathrm{~T} /$ SIGMA $) \# \# A L P H A))$;

$\mathrm{S} 1=\mathrm{SUM}(\mathrm{C} \# \mathrm{LOG}(\mathrm{T}))$; 
S2=SUM(C\#LOG(1-EXP(-(T/SIGMA)\#\#ALPHA )));

S3=SUM((1-C)\#(1-(1-EXP(-(T/SIGMA)\#\#ALPHA))\#\#THETA));

$\mathrm{L} 1=-\mathrm{R} * \mathrm{ALPHA} * \mathrm{LOG}(\mathrm{SIGMA})+\mathrm{R} * \mathrm{LOG}(\mathrm{ALPHA})-\mathrm{S} 0+\mathrm{ALPHA} * \mathrm{~S} 1+(\mathrm{THETA}-1) * \mathrm{~S} 2+\mathrm{S} 3$

$\mathrm{L} 2=\mathrm{R} * \mathrm{LOG}(\mathrm{THETA})+$ THET.A $* \mathrm{~S} 2+\mathrm{S} 3 ;$

$\mathrm{L} 3=-\mathrm{R}^{*} \mathrm{ALPHA} * \mathrm{LOG}(\mathrm{SIGMA})-\mathrm{S} 0+(\mathrm{THETA}-1) * \mathrm{~S} 2+\mathrm{S} 3$;

FINISH;

/* a1, a2 e a3 são os valores iniciais e cadeia e n1 é comprimento de cada cadeia. */

START NORM;

AALPHA $=\mathrm{J}(\mathrm{N} 1,1,.) ; \mathrm{TTHETA}=$ AALPHA;SSIGMA=TTHETA;

AALPHA $[1]=A 1 ;$ TTHETA[1]=A2; SSIGMA[1]=A3;

DO J=2 TO N1;

$\mathrm{ALPHA}=\mathrm{AALPHA}[\mathrm{J}-1]$;

THETA $=$ TTHETA[J-l];

SIGMA=SSIGMA[J-1];

RUN INI;

$\mathrm{DEN} 1=\mathrm{L} 1$;

ALP=RANGAM(199,ALP1)/ALP2;

$\mathrm{ALPHA}=\mathrm{ALP}$

THETA $=$ TTHETA[J-1];

$\mathrm{SIGMA}=\mathrm{SSIGMA}[\mathrm{J}-1]$;

RUN INI;

$\mathrm{NUM} 1=\mathrm{L} 1$

DEN2=L2;

D1=NUM1-DEN1; 
IF D1>=700 THEN D1=500;

ELSE D1=D1;

$\mathrm{P} 1=\mathrm{MIN}(\mathrm{D} 1,1)$;

IF P1>=RANUNI(09) THEN AALPHA[J]=ALP;

ELSE AALPHA[J]=AALPHA[J-1];

THET=RANGAM(1134,THET1)/THET2;

$\mathrm{ALPHA}=\mathrm{AALPHA}[\mathrm{J}]$;

THETA $=$ THET;

SIGMA =SSIGMA[J-1];

RUN INI;

NUM2=L2;

DEN3=L3;

D2=NUM2-DEN2;

IF D2 $>=700$ THEN D2 $=500$;

ELSE D2=D2;

$\mathrm{P} 2=\mathrm{MIN}(\mathrm{D} 2,1)$;

IF $\mathrm{P} 2>=$ RANUNI(10) THEN TTHETA[J]=THET;

ELSE TTHETA[J]=TTHETA[J-1];

$\mathrm{SIG}=\mathrm{RANGAM}(2434, \mathrm{SIG} 1) / \mathrm{SIG} 2$;

ALPHA =AALPHA[J];

THETA $=$ TTHETA[J];

SIGMA =SIG;

RUN INI;

NUM3=L3; 
D3=NUM3-DEN3;

$\mathrm{P} 3=\mathrm{MIN}(\mathrm{D} 3,1)$;

IF P3>=RANUNI(102) THEN SSIGMA[J]=SIG;

ELSE SSIGMA $[\mathrm{J}]=$ SSIGMA $[\mathrm{J}-1]$;

END;

$\mathrm{BB} 0=\mathrm{AALPHA}[(\mathrm{N} 1 / 2+1): \mathrm{N} 1]$;

$\mathrm{BB} 1=\mathrm{TTHETA}[(\mathrm{N} 1 / 2+1): \mathrm{N} 1]$;

$\mathrm{BB} 2=\operatorname{SSIGMA}[(\mathrm{N} 1 / 2+1): \mathrm{N} 1]$;

FINISH:

2. Módulo para obtenção de amostras de $\beta_{0}, \beta_{1}, \delta$ e $\theta$ no modelo de regressão Weibullexponenciada utilizando o algoritmo de Gibb'com Metropolis-Hasting para os dados de Nelson (1990).

PROC IML;

START INI;

USE A;

READ ALL VAR $\{Y$ X CENSURA $\}$;

$\mathrm{N}=\mathrm{NROW}(\mathrm{Y})$;

$\mathrm{R}=\mathrm{SUM}(\mathrm{CENS})$

$\mathrm{Z}=(\mathrm{Y}-\mathrm{B} 0-\mathrm{B} 1 * \mathrm{X}) / \mathrm{DELTA}$

$\mathrm{F} 1=1-\operatorname{EXP}(-\operatorname{EXP}(\mathrm{Z}))$

$\mathrm{S}=\mathrm{SUM}(\mathrm{CENS} \# \mathrm{LOG}(\mathrm{F} 1))$;

S1=SUM((1-CENS)\#LOG(1-F1\#\#THETA));

$\mathrm{L} 1=\mathrm{R} * \mathrm{LOG}(\mathrm{THETA})+\mathrm{THETA}{ }^{*} \mathrm{~S}+\mathrm{S} 1$;

L2=-R*LOG(DELTA $)+\operatorname{SUM}($ CENS\#Z)-SUM(CENS\#EXP(Z))+(THETA-1)*S+S1; 
$\mathrm{L} 3=-\mathrm{R} * \mathrm{~B} 0 /$ DELTA-SUM $(\mathrm{CENS} \# \operatorname{EXP}(\mathrm{Z}))+($ THETA-1 $) *$ S+S1 ;

$\mathrm{L} 4=-\mathrm{B} 1 * \mathrm{SUM}(\mathrm{CENS} \# \mathrm{X} / \mathrm{DELTA})-\mathrm{SUM}(\mathrm{CENS} \# \operatorname{EXP}(\mathrm{Z}))+(\mathrm{THETA}-1) * \mathrm{~S}+\mathrm{S} 1$;

FINISH;

/ X1, X2 e X3 são os valores iniciais e N1 é o comprimento de cada cadeia. */

START NORM;

$\mathrm{BB} 0=\mathrm{J}(\mathrm{N} 1,1,.) ; \mathrm{BB} 1=\mathrm{BB} 0 ; \mathrm{DDELTA}=\mathrm{BB} 1 ; \mathrm{TTHETA}=\mathrm{J}(\mathrm{N} 1,1,$.

$\mathrm{BB} 0[1]=\mathrm{X} 1 ; \mathrm{BB} 1[1]=\mathrm{X} 2 ; \mathrm{DDELTA}[1]=\mathrm{X} 3 ; \mathrm{TTHETA}[1]=\mathrm{X} 4$;

DO $\mathrm{J}=2$ TO N1;

$\mathrm{B} 0=\mathrm{BB} 0[\mathrm{~J}-1]$;

$\mathrm{B} 1=\mathrm{BB} 1[\mathrm{~J}-1]$

DELTA $=$ DDELTA $[\mathrm{J}-1]$;

THETA $=$ TTHETA[J-1];

RUN INI;

DEN1=L3;

BETA0 $=$ BB0 $[J-1]+S I G 1 *$ RANNOR(19);

$\mathrm{B} 0=\mathrm{BETA} 0$;

$\mathrm{B} 1=\mathrm{BB} 1[\mathrm{~J}-1]$;

DELTA=DDELTA[J-1];

THETA $=$ TTHETA[J-1];

RUN INI;

NUM1=L3;

DEN2=L4;

D1=NUM1-DEN1;

IF D1 $<=700$ THEN D1=D1; 
ELSE D1=500;

$\mathrm{Q} 1=\operatorname{EXP}(\mathrm{D} 1)$

$\mathrm{P} 1=\operatorname{MIN}(\mathrm{Q} 1,1)$

IF P1>=RANUNI(09) THEN BB0[J]=B0;

ELSE BB0[J]=BB0[J-1];

$\mathrm{BETA} 1=\mathrm{BB}[\mathrm{J}-1]+\mathrm{SIG} 2 \times{ }^{\times} \mathrm{ANNOR}(13) ;$

$\mathrm{B} 0=\mathrm{BB} 0[\mathrm{~J}]$

$\mathrm{B} 1=\mathrm{BETA} 1$

DELTA $=$ DDELTA[J-1];

THETA $=$ TTHETA[J-1]:

RUN INI;

$\mathrm{NUM} 2=\mathrm{L} 4$;

DEN3=L2;

D2=NUM2-DEN2;

IF D2 $<=700$ THEN D2=D2;

ELSE D2=500;

$\mathrm{Q} 2=\operatorname{EXP}(\mathrm{D} 2) ;$

$\mathrm{P} 2=\operatorname{MIN}(\mathrm{Q} 2,1)$

IF P2>=RANUNI(10) THEN BB1[J]=B1;

ELSE BB1[J]=BB1[J-1];

DEL=RANGAM(123,DEL1)/DEL2;

$\mathrm{B} 0=\mathrm{BB} 0[\mathrm{~J}]$

$\mathrm{B} 1=\mathrm{BB} 1[\mathrm{~J}]$

DELTA $=$ DEL; 
THETA $=$ TTHETA[J-1];

RUN INI;

NUM3 $=$ L2;

DEN4=L1;

D3=NUM3-DEN3;

IF D3<=700 THEN D3=D3;

ELSE D3=500;

$\mathrm{Q} 3=\mathrm{EXP}(\mathrm{D} 3)$;

$\mathrm{P} 3=\mathrm{MIN}(\mathrm{Q} 3,1)$;

IF P3>=RANUNI(102) THEN DDELTA[J]=DEL;

ELSE DDELTA[J]=DDELTA[J-1];

THE=RANGAM(112,THE1)/THE2;

$\mathrm{B} 0=\mathrm{BB} 0[\mathrm{~J}]$;

$\mathrm{B} 1=\mathrm{BB} 1[\mathrm{~J}]$;

DELTA=DDELTA $[\mathrm{J}]$;

THETA=THE;

RUN INI;

NUM4=L1;

D4=NUM4-DEN4;

IF D4 $<=700$ THEN D4=D4;

ELSE D4=500;

$\mathrm{Q} 4=\operatorname{EXP}(\mathrm{D} 4)$;

$\mathrm{P} 4=\operatorname{MIN}(\mathrm{Q} 4,1)$;

IF P4>=RANUNI(102) THEN TTHETA[J]=THE; 
ELSE TTHETA $[J]=$ TTHETA[J-1];

END;

$\mathrm{BB} 0=\mathrm{BB} 0[(\mathrm{~N} 1 / 2+1): \mathrm{N} 1] ;$

$\mathrm{BB} 1=\mathrm{BB} 1[(\mathrm{~N} 1 / 2+1): \mathrm{N} 1]$;

DDELTA=DDELTA[(N1/2+1):N1];

TTHETA $=$ TTHETA $[(\mathrm{N} 1 / 2+1): \mathrm{N} 1]$;

FINISH:

3. Modulo para gerar amostras de $\alpha_{11}, \alpha_{21}, \alpha_{12}$ e $\alpha_{22}$, sob $H_{1}$ na comparação duas amostras exponenciais com dados acelerados utilizando o algoritmo de Gibbs com Metropolis-Hasting.

PROC IML;

START INI;

$\mathrm{R}=\{4,6,7,8,9\} ;$

$\mathrm{R} 1=\mathrm{R}[+]$;

$V=\{5,10,15,20,25\}$;

$\mathrm{A} 11=\{291.9,76.67,67.29,51.75,71.39\} ;$

$\mathrm{A} 12=\{271.34,79.83,74.14,54.75,91.94\} ;$

S11=SUM(R\#A11\#(V\#\#ALPHA21));

S12=SUM(R\#A12\#(V\#\#ALPHA22));

$\mathrm{L} 21=\mathrm{ALPHA} 21 *(\mathrm{SUM}(\mathrm{R} \# \mathrm{LOG}(\mathrm{V}))+4 * \mathrm{LOG}(5)+9 * \mathrm{LOG}(25))$

-S11/ALPHA11+ALPHA21**2/2000;

L22=ALPHA22* $(\operatorname{SUM}(\mathrm{R} \# \mathrm{LOG}(\mathrm{V}))+4 * \mathrm{LOG}(5)+9 * \mathrm{LOG}(25))$

-S12/ALPHA12+ALPHA22**2/2000;

FINISH; 
$/^{*} \mathrm{X}[1], \mathrm{X}[2], \mathrm{X}[3]$ e X[4] são os valores iniciais para cada cadeia e n1 é comprimento de cada cadeia. ${ }^{*} /$

START METROP;

$\mathrm{X} 11=\mathrm{J}(\mathrm{N} 1,1,.) ; \mathrm{X} 21=\mathrm{X} 11 ; \mathrm{X} 12=\mathrm{X} 21 ; \mathrm{X} 22=\mathrm{X} 12 ;$

$\mathrm{X} 11[1]=\mathrm{X}[1] ; \mathrm{X} 21[1]=\mathrm{X}[2] ; \mathrm{X} 12[1]=\mathrm{X}[3] ; \mathrm{X} 22[1]=\mathrm{X}[4]$;

DO J=2 TO N1;

ALPHA11=X11[J-1];

ALPHA21=X21[J-1];

ALPHA12=X12[J-1];

ALPHA22=X22[J-1];

RUN INI;

AL11=S11/RANGAM(4234,R1+13);

ALPHA11=AL11;

ALPHA21 $=$ X21[J-1];

ALPHA12=X12[J-1];

ALPHA22=X22[J-1];

RUN INI;

DEN21=L21;

AL21 $=$ SIG1*RANNOR(1234)+X21[J-1];

ALPHA11=AL11;

ALPHA21=AL21;

ALPHA12=X12[J-1];

ALPHA22=X22[J-1];

RUN INI; 
NUM21=L21;

D21=NUM21-DEN21;

IF D21 $<=700$ THEN D21=D21;

ELSE D21=500;

$\mathrm{P} 21=\operatorname{MIN}(1, \operatorname{EXP}(\mathrm{D} 21))$;

IF P21>=RANUNI(1234) THEN AL21=AL21;

ELSE AL21=X21[J-1];

ALPHA11=AL11:

ALPHA21=AL21;

ALPHA $12=\mathrm{X} 12[\mathrm{~J}-1]$

ALPHA22=X22[J-1];

RUN INI;

$\mathrm{AL} 12=\mathrm{S} 12 / \mathrm{RANGAM}(1234, \mathrm{R} 1+13)$;

ALPHA11=AL11;

ALPHA21=AL21;

ALPHA $12=A L 12 ;$

ALPHA22=X22[J-1];

RUN INI;

DEN22=L22;

AL22 $=$ SIG $2 *$ RANNOR $(1234)+X 22[\mathrm{j}-1]$;

ALPHA11=AL11;

ALPHA21=AL21;

ALPHA12=AL12;

ALPHA22=AL22; 
RUN INI;

NUM22=L22;

D22=NUM22-DEN22;

IF D $22<=700$ THEN D22=D22;

ELSE D22=500;

$\mathrm{P} 22=\mathrm{MIN}(1, \operatorname{EXP}(\mathrm{D} 22))$;

IF P22>=RANUNI(999) THEN AL22=AL22;

ELSE AL22=X22[J-1];

$\mathrm{X} 11[\mathrm{~J}]=\mathrm{AL} 11 ;$

$\mathrm{X} 21[\mathrm{~J}]=\mathrm{AL} 21 ;$

$\mathrm{X} 12[\mathrm{~J}]=\mathrm{AL} 12 ;$

$\mathrm{X} 22[\mathrm{~J}]=\mathrm{AL} 22 ;$

END;

$\mathrm{X} 11=\mathrm{X} 11[(\mathrm{~N} 1 / 2+1): \mathrm{N} 1] ;$

$\mathrm{X} 21=\mathrm{X} 21[(\mathrm{~N} 1 / 2+1): \mathrm{N} 1]$;

$\mathrm{X} 12=\mathrm{X} 12[(\mathrm{~N} 1 / 2+1): \mathrm{N} 1]$;

$\mathrm{X} 22=\mathrm{X} 22[(\mathrm{~N} 1 / 2+1): \mathrm{N} 1] ;$

FINISH;

4. Modulo para a obtenção das amostras $\lambda$ e $p$ no modelo de mistura exponencial utilizando o algoritmo de Gibbs com Metropolis-Hasting.

PROC IML;

USE A;

READ ALL VART C;

START INI; 
$\mathrm{R}=\mathrm{SUM}(\mathrm{C})$;

$\mathrm{P}=\operatorname{EXP}(\mathrm{BETA}) /(1+\operatorname{EXP}(\mathrm{BETA})) ;$

$\mathrm{S}=\operatorname{SUM}\left((1-\mathrm{C}) \# \mathrm{LOG}\left(1-\mathrm{P}+\mathrm{P} * \operatorname{EXP}\left(-\mathrm{T}^{*} \operatorname{EXP}(\mathrm{LAMBDA})\right)\right)\right)$;

$\mathrm{L} 1=(\mathrm{R}+1) * \mathrm{LOG}(\mathrm{P})-0.5 * \mathrm{LOG}(1-\mathrm{P})+\mathrm{S}-\mathrm{LOG}(1+\mathrm{EXP}(\mathrm{BETA}))$;

$\mathrm{L} 2=(\mathrm{R}+2) * \mathrm{LAMBDA}-\mathrm{SUM}(\mathrm{C} \# \mathrm{~T}) * \operatorname{EXP}(\mathrm{LANBDA})+\mathrm{S}$;

FINISH;

START METROP;

$\mathrm{LAMB}=\mathrm{J}(\mathrm{N} 1,1,.) ; \mathrm{BBETA}=\mathrm{J}(\mathrm{N} 1,1,$.$) ;$

$\mathrm{LAMB}[1]=\mathrm{X}[1] ; \mathrm{BBETA}[1]=\mathrm{X}[2]$;

DO J=2 TO N1;

LAMBDA $=$ LAMB[J-1];

$\mathrm{BETA}=\mathrm{BBETA}[\mathrm{J}-1]$;

RUN INI;

$\mathrm{DEN} 1=\mathrm{L} 1 ;$

BETA $1=$ BBETA $[\mathrm{J}-1]+$ RANNOR $(234)^{*}$ SIG1;

LAMBDA=LAMB[J-1];

$\mathrm{BETA}=\mathrm{BETA} 1 ;$

RUN INI;

NUM1=L1;

$\mathrm{DEN} 2=\mathrm{L} 2$;

D1=NUM1-DEN1;

IF D1>=700 THEN D1=500;

ELSE D1=D1;

$\mathrm{Q} 1=\operatorname{EXP}(\mathrm{D} 1)$ 
$\mathrm{P} 1=\operatorname{MIN}(1, \mathrm{Q} 1)$

IF P1>=RANUNI(123) THEN BBETA[J]=BETA1;

ELSE BBETA[J]=BBETA[J-1];

LAM1=LAMB[J-1]+RANNOR(13455)*SIG2;

LAMBDA=LAM1;

$\mathrm{BETA}=\mathrm{BBETA}[\mathrm{J}]$;

RUN INI;

NUM2=L2;

D2=NUM2-DEN2;

IF D2>=700 THEN D2=500;

ELSE D2=D2;

$\mathrm{Q} 2=\operatorname{EXP}(\mathrm{D} 2)$;

$\mathrm{P} 2=\operatorname{MIN}(1, \mathrm{Q} 2)$;

IF P2>=RANUNI(9901) THEN LAMB[J]=LAM1;

ELSE LAMB[J]=LAMB[J-1];

END;

$\mathrm{B} 1=\operatorname{EXP}(\operatorname{BBETA}[(\mathrm{N} 1 / 2+1): \mathrm{N} 1]) /(1+\operatorname{EXP}(\operatorname{BBETA}[(\mathrm{N} 1 / 2+1): \mathrm{N} 1]))$;

$\mathrm{B} 2=\operatorname{EXP}(\operatorname{LAMB}[(\mathrm{N} 1 / 2+1): \mathrm{N} 1]) ;$

FINISH;

5. Modulo para gerar amostras de $\alpha_{0}, \alpha_{1}, \beta_{1}$ e $\beta_{2}$, no modelo de mistura exponencial com covariáveis.

PROC IML;

USE A;

READ ALL VAR $\{$ T C X $\}$; 
START INI;

$\mathrm{R}=\mathrm{SUM}(\mathrm{C})$;

LAMBDA $=$ EXP $($ ALPHA0+ALPHA $1 \# X)$;

$\mathrm{P}=\operatorname{EXP}\left(\mathrm{BETA} 0+\mathrm{BETA} 1^{*} \mathrm{X}\right) /\left(1+\operatorname{EXP}\left(\mathrm{BETA} 0+\mathrm{BETA} 1^{*} \mathrm{X}\right)\right)$;

PSI1=SUM $((1-C) \# L O G(1-P+P \# \operatorname{EXP}(-L A M B D A \# T)))$;

$\mathrm{PSI}=\mathrm{SUM}(\mathrm{C} \# \operatorname{LOG}(\mathrm{P}))$;

$\mathrm{S} 1=\mathrm{SUM}(\mathrm{C} \# \mathrm{~T} \#$ LAMBDA $)$;

L0=ALPHA0*R-S1-0.5*ALPHA0**2/1000000+ PSI1;

L1=SUM(ALPHA1\#X\#C)-S1-0.5*ALPHA1**2/1000000+PSI1;

$\mathrm{LL} 0=-0.5 * \mathrm{BETA}^{*} * 2 / 100000+\mathrm{PSI} 1+\mathrm{PSI} 2 ;$

$\mathrm{LL} 1=-0.5 * \mathrm{BETA}^{*} * 2 / 100000+\mathrm{PS} I 1+\mathrm{PSI} 2 ;$

FINISH;

START METROP;

AALPHA0 $=\mathrm{J}(\mathrm{N} 1,1,$.$) ; AALPHA 1=\mathrm{J}(\mathrm{N} 1,1,.) ; \mathrm{BBETA} 0=\mathrm{J}(\mathrm{N} 1,1,.) ; \mathrm{BBETA} 1=\mathrm{J}(\mathrm{N} 1,1,$.$) ;$

AALPHA0[1]=A1; AALPHA1[1]=A2; BBETA0[1]=B1;BBETA1[1]=B2;

DO J=2 TO N1;

ALPHA0=AALPHA0[J-1];

ALPHA1=AALPHA1[J-1];

BETA0 $=$ BBETA0[J-1];

BETA $1=\operatorname{BBETA} 1[\mathrm{~J}-1]$;

RUN INI;

DEN1=L0;

AL0=AALPHA0[J-1]+RANNOR(12323)*SIG1;

$\mathrm{ALPHA} 0=\mathrm{AL} 0$; 
ALPHA1=AALPHA1[J-1];

$\mathrm{BETA} 0=\mathrm{BBETA} 0[\mathrm{~J}-1]$;

BETA $1=\operatorname{BBETA} 1[\mathrm{~J}-1]$;

RUN INI;

NUM1 $=$ L0;

$\mathrm{DEN} 2=\mathrm{L} 1 ;$

D1=NUM1-DEN1;

IF D1>=700 THEN D1=500;

ELSE D1=D1;

$\mathrm{Q} 1=\mathrm{EXP}(\mathrm{D} 1) ;$

$\mathrm{P} 1=\mathrm{MIN}(1, \mathrm{Q} 1)$;

IF P1>=RANUNI(123) THEN AALPHA0[J]=AL0;

ELSE AALPHA0[J]=AALPHA0[J-1];

AL1=AALPHA1[J-1]+RANNOR(13455)*SIG2;

ALPHA0 $=$ AALPHA0[J];

ALPHA1=AL1;

BETA0 $=$ BBETA0[J-1];

BETA1 $=$ BBETA1 $[\mathrm{J}-1]$;

RUN INI;

NUM2=L1;

DEN3=LL0;

D2=NUM2-DEN2;

IF D2>=700 THEN D2=500;

ELSE D2=D2; 
$\mathrm{Q} 2=\operatorname{EXP}(\mathrm{D} 2)$;

$\mathrm{P} 2=\mathrm{MIN}(1, \mathrm{Q} 2)$;

IF P2>=RANUNI(9901) THEN AALPHA1[J]=AL1;

ELSE AALPHA1[J]=AALPHA1[J-1];

$\mathrm{BE} 0=\mathrm{BBETA} 0[\mathrm{~J}-1]+\mathrm{RANNOR}(656)^{*} \mathrm{SIG} 2$;

ALPHA0=AALPHA0[J];

ALPHA1=AALPHA1[J];

BETA $0=\mathrm{BE} 0$;

BETA $1=$ BBETA1[J-1];

RUN INI;

NUM $3=$ LL 0 ;

DEN4=LL1;

D3=NUM3-DEN3;

IF D3 $>=700$ THEN D3=500;

ELSE D3=D3;

$\mathrm{Q} 3=\mathrm{EXP}(\mathrm{D} 3)$;

$\mathrm{P} 3=\mathrm{MIN}(1, \mathrm{Q} 3)$;

IF P3>=RANUNI(1020) THEN BBETA0[J]=BE0;

ELSE BBETA0[J]=BBETA0[J-1];

BE1=BBETA1[J-1]+RANNOR $(656)^{*}$ SIG2;

ALPHA0=AALPHA0[J];

ALPHA1=AALPHA1[J];

BETA0 $=$ BBETA0[J];

BETA1=BE1; 
RUN INI;

NUM4=LL1;

D4=NUM4-DEN4;

IF D4>700 THEN D4=500;

ELSE D4=D4;

$\mathrm{Q} 4=\mathrm{EXP}(\mathrm{D} 4)$;

$\mathrm{P} 4=\mathrm{MIN}(1, \mathrm{Q} 4)$;

IF P4>=RANUNI(1020) THEN BBETA1[J]=BE1;

ELSE BBETA1[J]=BBETA1[J-1];

END;

$\mathrm{AA} 0=\mathrm{AALPHA} 0[(\mathrm{~N} 1 / 2+1): \mathrm{N} 1]$;

$\mathrm{AA} 1=\mathrm{AALPHA} 1[(\mathrm{~N} 1 / 2+1): \mathrm{N} 1]$;

$\mathrm{BB} 0=\mathrm{BBETA} 0[(\mathrm{~N} 1 / 2+1): \mathrm{N} 1]$;

$\mathrm{BB} 1=\mathrm{BBETA} 1[(\mathrm{~N} 1 / 2+1): \mathrm{N} 1]$;

FINISH;

6. Programa desenvolvido no pacote $\mathrm{Ox}$ para obtenção das estimativas de maxima verossimilhança e o valor da estatística para testar $H_{0}: p=1$ versus $H_{1}: p<1$ no modelo de mistura exponencial.

\#include<oxstd.h>

\#include< oxdraw.h $>$

\#include<oxfloat.h $>$

\#include $<$ maximize.h $>$

\#include $<$ simula.h $>$

\#pragma link("maximize.oxo") 
static decl g_mY;

log_vero(const vP,const adFunc, const avScore,const amHessian)

\{

$\operatorname{decl} \mathrm{n}=$ rows $\left(\mathrm{g} \_\mathrm{mY}\right)$;

decl cont,t,c;

$\operatorname{decl}$ uns $=$ ones $(\mathrm{n}, 1)$;

decl vero=zeros $(1, n)$;

$\operatorname{decl} \mathrm{a}=\mathrm{fabs}(\mathrm{vP}[0][0]) ;$

$\operatorname{decl} \mathrm{p}=$ fabs $(\mathrm{vP}[1][0])$;

for $($ cont $=0 ;$ cont $<\mathrm{n} ;++$ cont $)\{$

$\mathrm{t}=\mathrm{g}-\mathrm{m} Y[\mathrm{cont}][0]$

$\mathrm{c}=\mathrm{g} \_\mathrm{mY}[$ cont $][1]$;

vero $[0][\operatorname{cont}]=c^{*}\left(\log (a)+\log (p)-a^{*} t\right)+(1-c)^{*} \log \left(1-p+p^{*} \exp \left(-1^{*} a^{*} t\right)\right)$;

\}

$\operatorname{adFunc}[0]=\operatorname{double}\left(\right.$ vero $^{\times}$uns $)$;

if(isnan(adFunc[0]))

return 0 ;

else

return 1 ;

\}

main()

\{

decl tempo=loadmat("a:tempo.dat",1);

decl censura=loadmat( "a:censura.dat",1); 
g_mY $=$ tempo|censura

$\operatorname{decl} \operatorname{lah} 0=\operatorname{sumc}\left(\mathrm{g} \_\mathrm{mY}[][1]\right) / \operatorname{sumc}\left(\mathrm{g} \_\mathrm{mY}[][0]\right)$;

$\operatorname{decl} \operatorname{lvh} 0=\operatorname{sumc}\left(\mathrm{g} \_\mathrm{mY}[][1]\right) * \log (\operatorname{lah} 0)-\operatorname{lah} 0 * \operatorname{sumc}\left(\mathrm{g} \_\mathrm{mY}[][0]\right) ;$

$\operatorname{print}\left(\right.$ rows $\left.\left(\mathrm{g} \_\mathrm{mY}\right), \mathrm{lah0}\right)$;

decl dfunc;

decl $v \mathrm{P}=<1 ; 0.6>$;

MaxControl(-1,20);

decl mhess $=0.000001 *$ unit $(2)$;

decl ir;

ir $=$ MaxBFGS(log_vero,\&vP,\&dfunc,\&mhess, 1$)$;

Num2Derivative(log_vero,vP,\&mhess);

if $(\mathrm{ir}==$ MAX_CONV $\| \mathrm{ir}==$ MAX_WEAK_CONV $)$

\{

print("Os EMV para modelo de mistura exponencial", vP);

decl hes $=((-1 /$ mhess $))$;

print("A matriz de covariáncia ",hes)

print("O valor da estatística do teste", $-2 *($ lvh0-dfunc));

\}

\} 